\title{
Mechanism and Origin of Chemoselectivity of Ru-Catalyzed Cross-Coupling of Secondary Alcohols to $\beta$-Disubstituted Ketones
}

\author{
Tian-Tian Liu, ${ }^{a}$ Shi-Ya Tang, ${ }^{\mathrm{b}}$ Bing Hu, ${ }^{\mathrm{a}}$ Peng Liu, ${ }^{\mathrm{a}}$ Siwei Bi, ${ }^{\mathrm{a}, *}$ Yuan-Ye Jiang, ${ }^{\mathrm{a}, *}$ \\ ${ }^{a}$ School of Chemistry and Chemical Engineering, Qufu Normal University, Qufu 273165, People's Republic of China \\ ${ }^{b}$ SINOPEC Research Institute of Safety Engineering, Qingdao, 266000, People's Republic of China.
}

\section{Corresponding Authors}

*E-mail: siweibi@126.com

*E-mail: yuanyejiang@qfnu.edu.cn 


\section{Table of Contents}

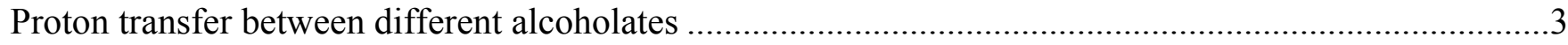

dehydration or intramolecular proton transfer from ab3 without tert-butanolate ......................................

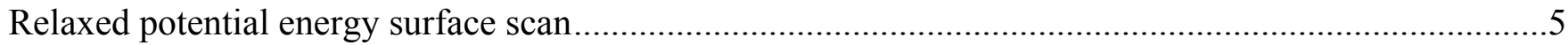

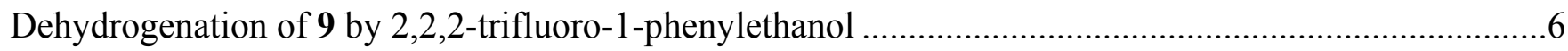

NCIplot of hydride transfer transition states ......................................................................................

Dehydrongenation of 9 by bulky alcohols and hydrogenation of bulky ketones on 9 ..............................

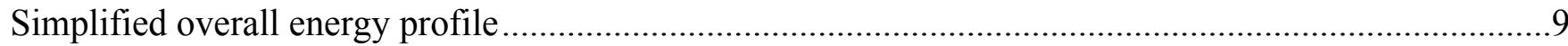

Energy barriers calculated by different geometry optimization methods .................................................11

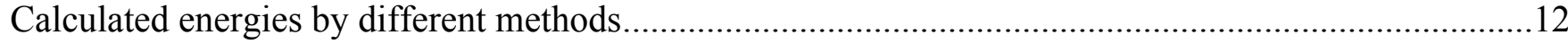

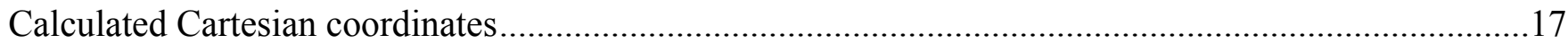




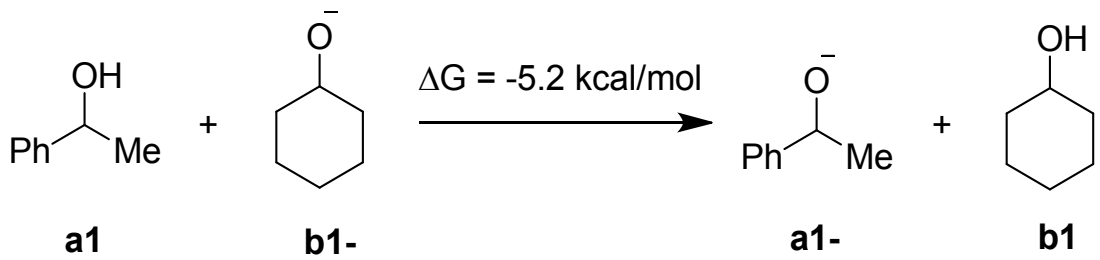

Scheme S1. Calculated solution-phase Gibbs free energies of proton transfer between different alcoholates (in $\mathrm{kcal} / \mathrm{mol}$ ) 


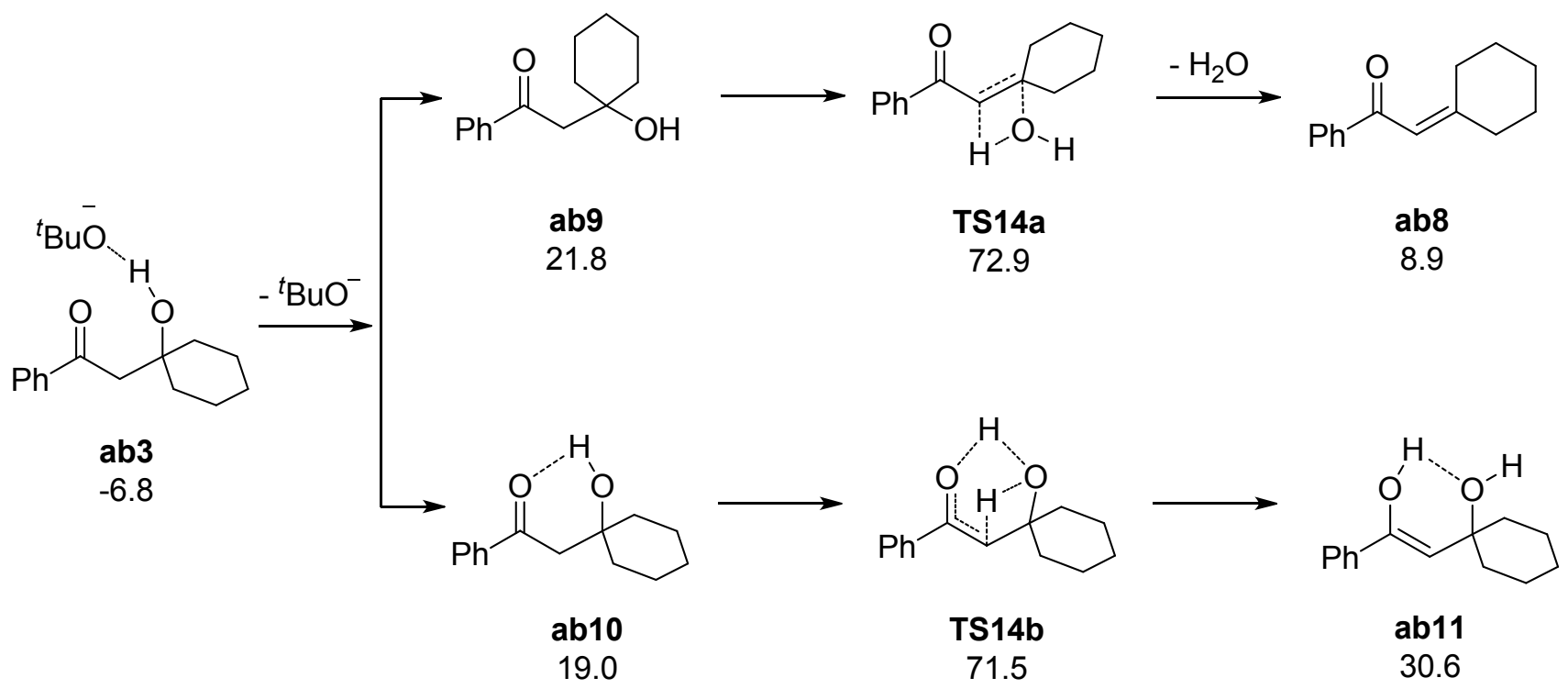

Scheme S2. Calculated solution-phase Gibbs free energies of dehydration or intramolecular proton transfer from ab3 without tert-butanolate (in $\mathrm{kcal} / \mathrm{mol}$ ) 


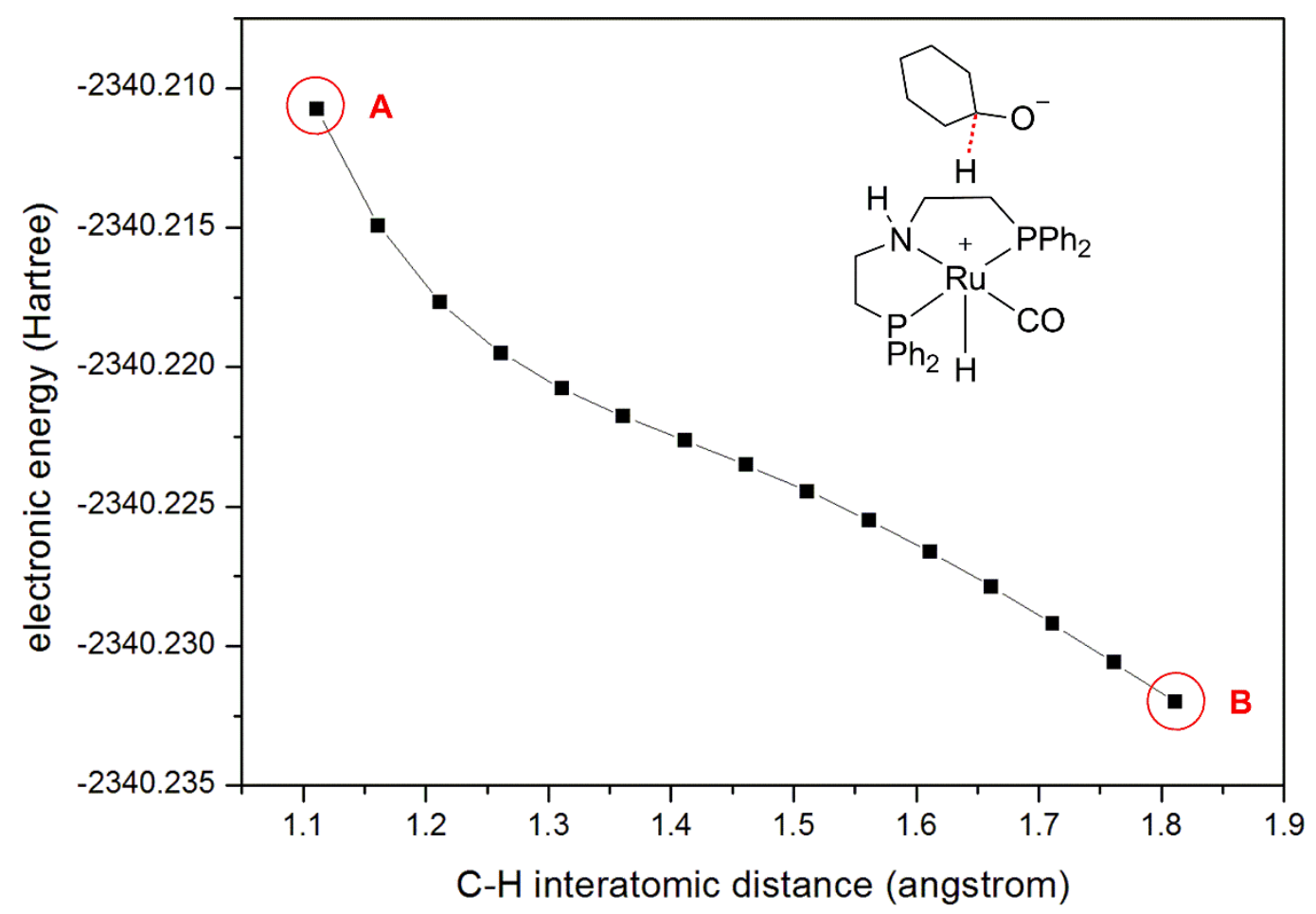

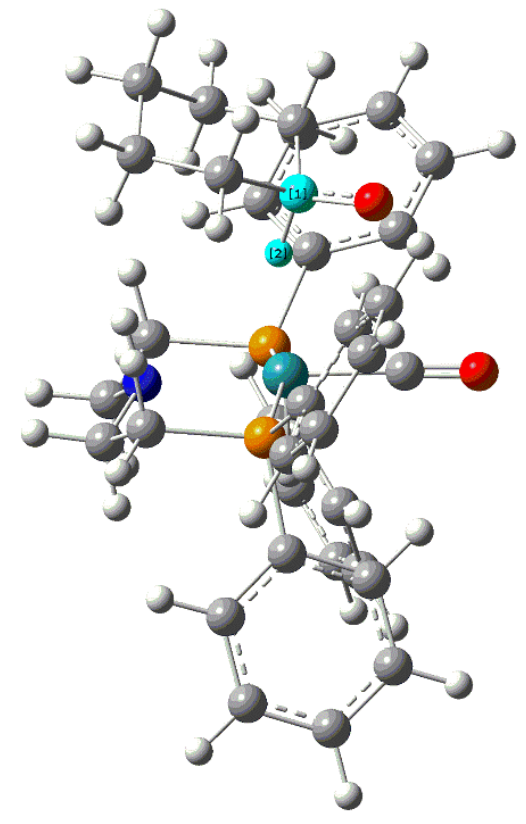

A

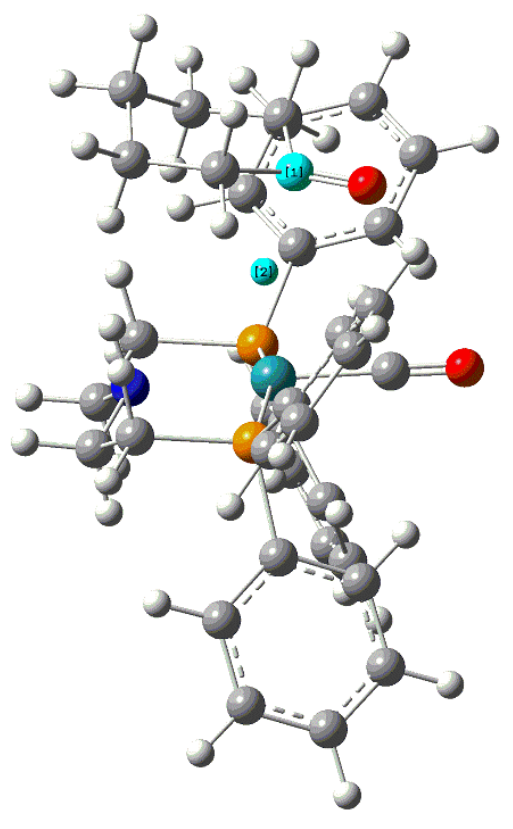

B

Figure S1. Relaxed potential energy surface scan of the $\mathrm{C}-\mathrm{H}$ interatomic distance and optimized structures. 


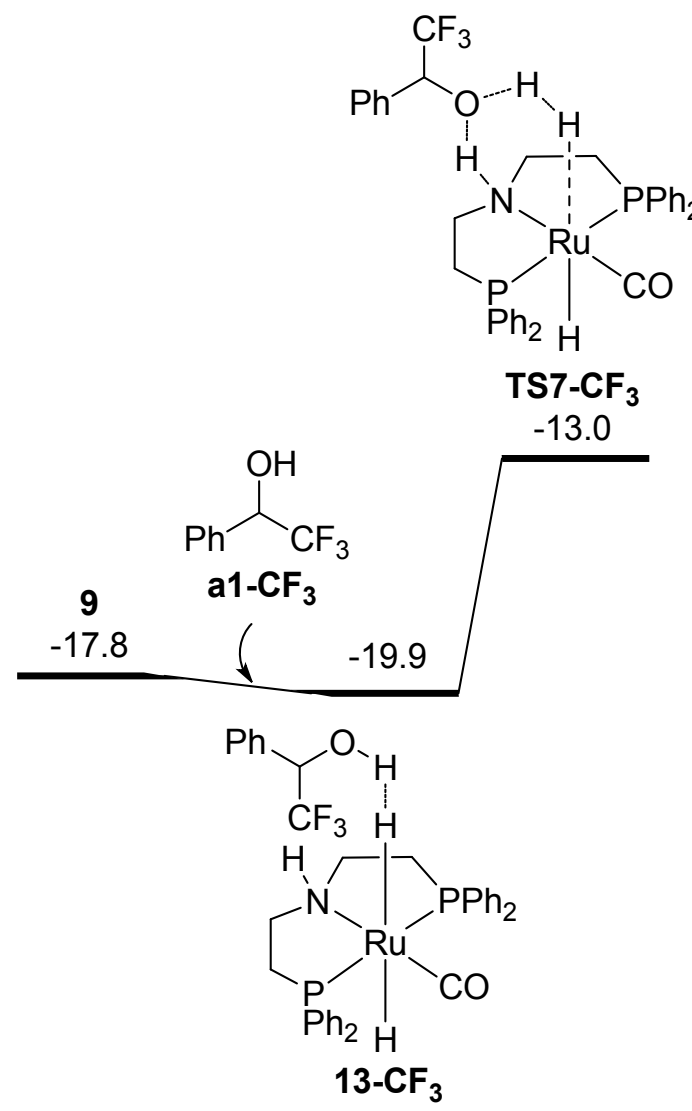

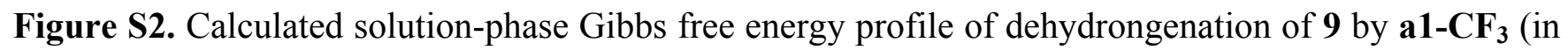
$\mathrm{kcal} / \mathrm{mol}$ ). 


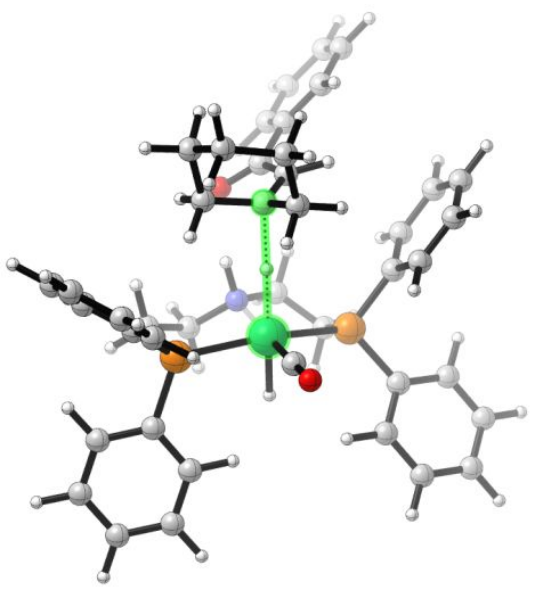

TS21

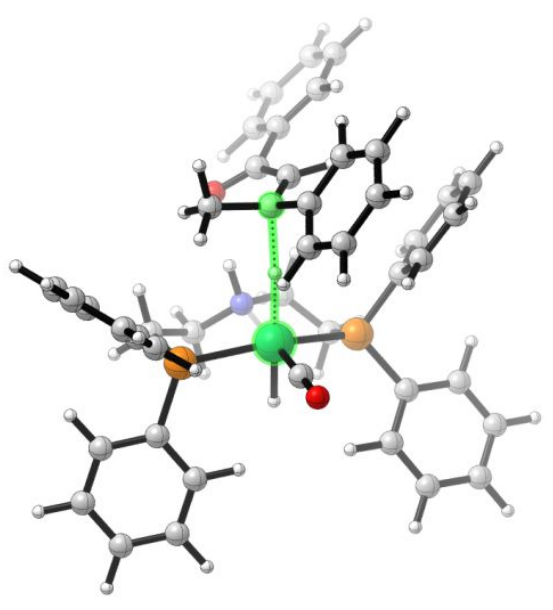

TS24

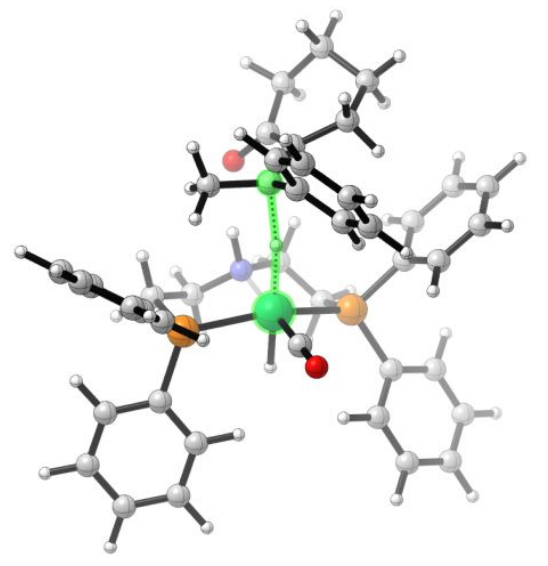

TS26

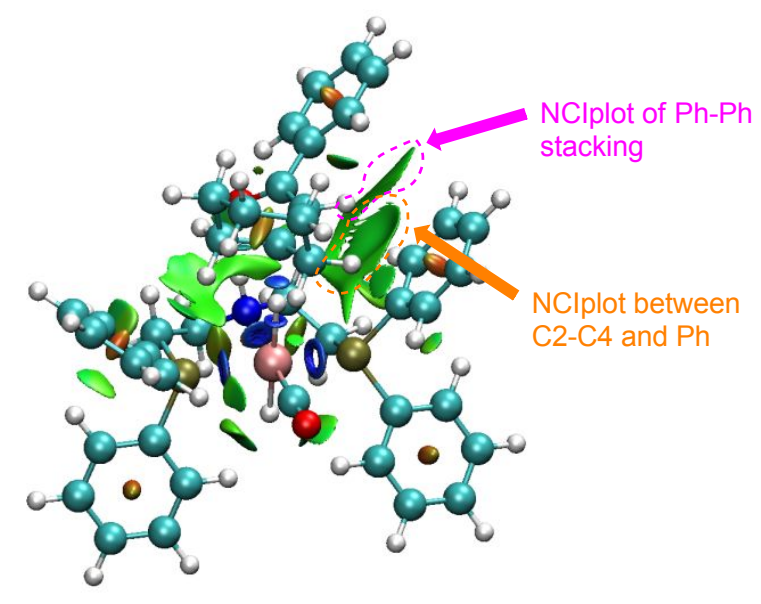

TS21

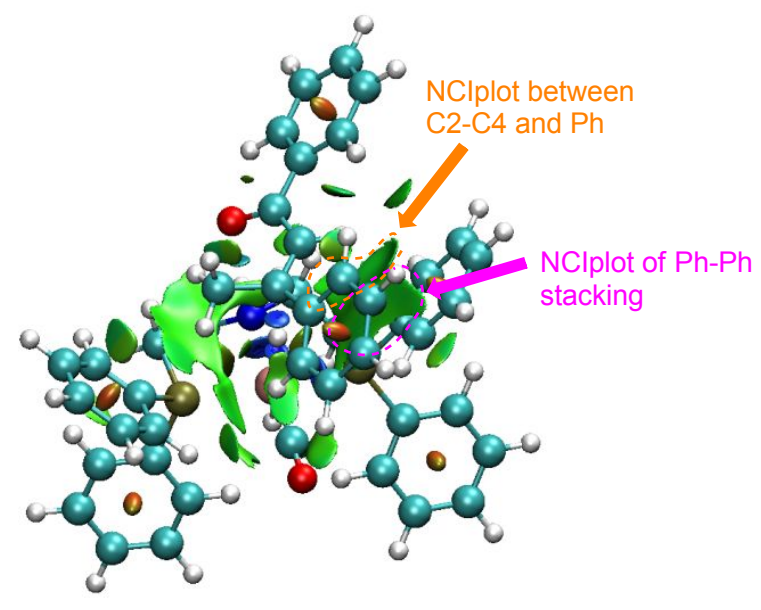

TS24

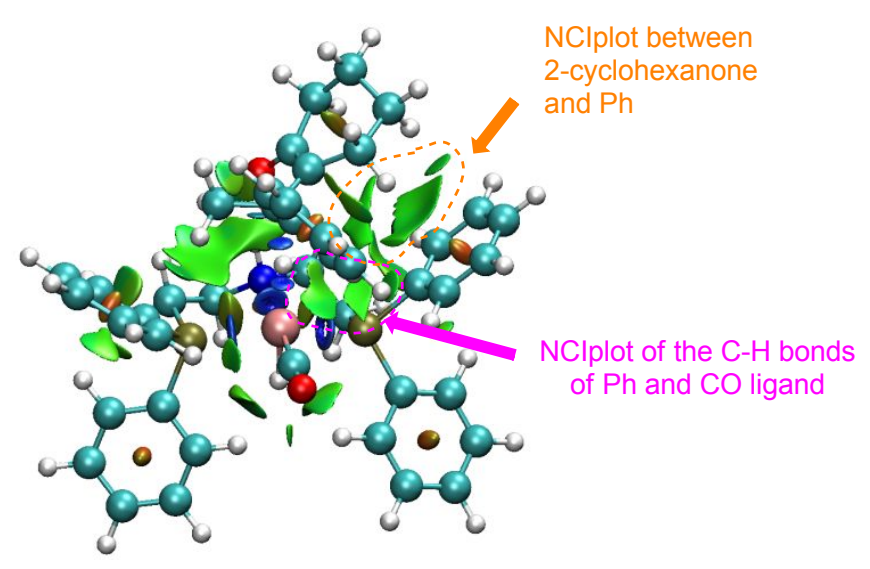

TS26

Figure S3. The plot of non-covalent interaction in hydride transfer transition states. Green areas indicate van der Waals interaction. Red areas indicate stronger destabilizing interaction. Blue areas indicate stronger stabilizing interaction. 

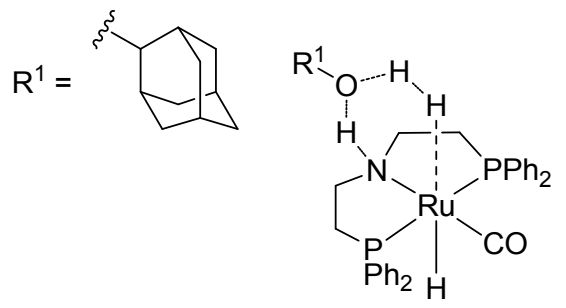

TS33

11.2

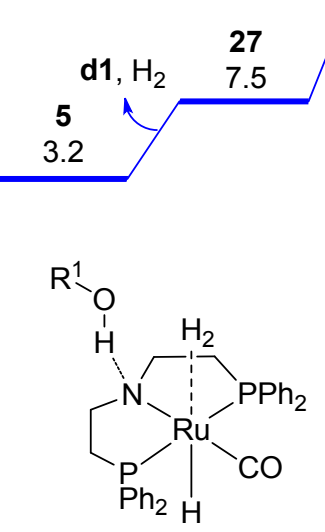

27

26

hydrogenation of cd1

d1-catalyzed dehydrogenation

$\mathrm{R}^{1-\mathrm{O}_{-}} \mathrm{H}$

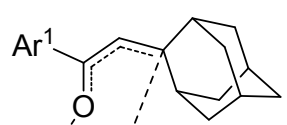<smiles>Cc1ccccc1</smiles>

$\mathrm{Ph}_{2} \mathrm{H}$

TS34

14.2<smiles>O=C(Br)C=C1C2CC3CC(C2)CC1C3</smiles>

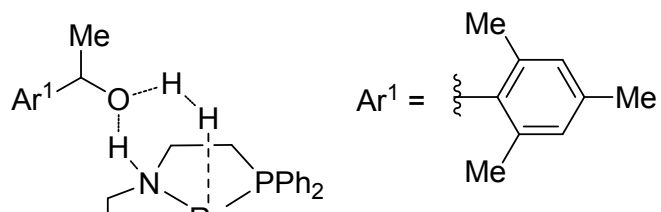

cd1

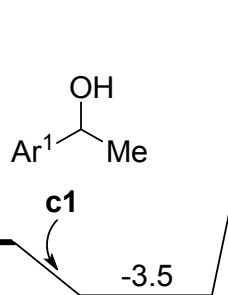

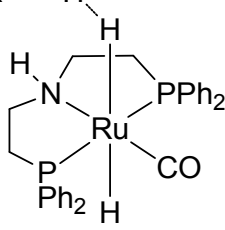

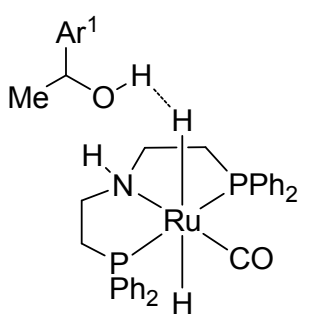

24

Figure S4. Calculated solution-phase Gibbs free energy profile of the dehydrongenation of 9 by $\mathbf{c 1}$ or d1 and the hydrogenation of $\mathbf{c d 1}$ (in $\mathrm{kcal} / \mathrm{mol}$ ). 

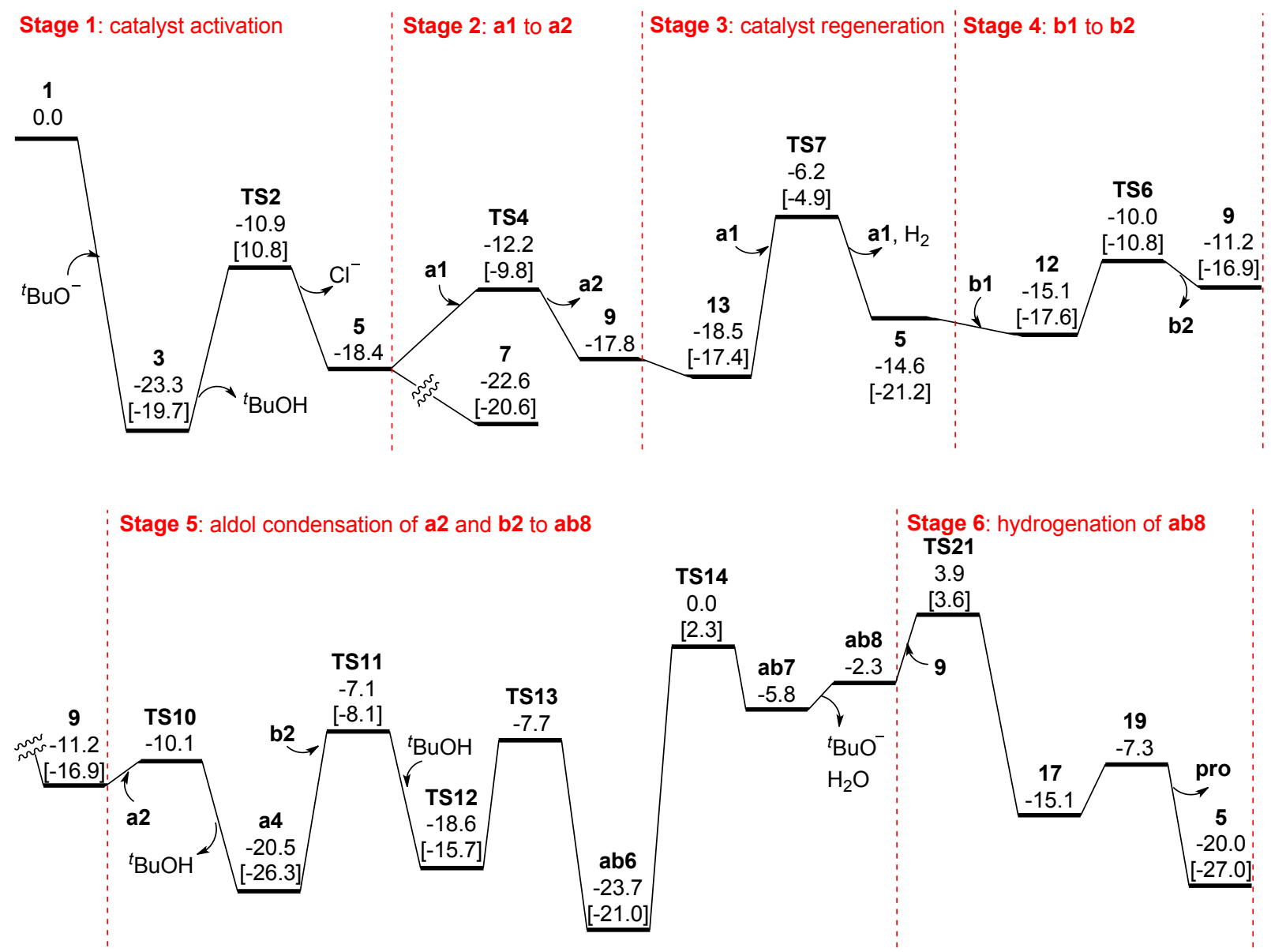

Figure S5. Simplified overall energy profile of the generation of pro (in $\mathrm{kcal} / \mathrm{mol}$ ). The relative solution-phase Gibbs free energies in brackets are calculated at $125^{\circ} \mathrm{C}$.

To determine the overall energy barrier, taking the energies of all starting materials as a reference point. As shown in Figure R3, the overall energy profile of the generation of pro from the starting materials can be simplified into six stages (some relative energies are different to these in the manuscript because the reference points are different). Stage $\mathbf{1}$ is the activation of catalyst to generate 5. Stage $\mathbf{2}$ is the dehydrogenation of $\mathbf{a} 1$ to $\mathbf{a} 2$ and ruthenium hydride intermediate 9. Stage 3 is the dihydrogen release of 9 to regenerate 5 which further catalyzes the dehydrogenation of b1 to b2 in Stage 4. After Stage 4, we now get one $\mathbf{a 2}$, one $\mathbf{b 2}$ and one 9. In the next Stage 5, the aldol condensation of $\mathbf{a} 2$ and $\mathbf{b 2}$ affords $\mathbf{a b 8}$. Then hydrogenation of ab8 on $\mathbf{9}$ occurs in Stage $\mathbf{6}$ to yield pro and regenerate 5 to start the next catalytic cycle.

It is noted that Stage 3 is irreversible as it generates dihydrogen and the reaction was conducted in an open system. In this case, the presence of stable intermediates like $\mathbf{3}$ and $\mathbf{7}$ no longer influence the overall energy barriers of following stages. Meanwhile, under the elevated reaction temperature, the aldol condensation is quasi-irreversible due to water evaporation but the accurate influence of the aldol condensation on the following hydrogenation from thermodynamics is unknown. According to the 
above analysis, the most possible rate-determining step might be the hydrogenation of ab8 via TS21 with a formal overall energy barrier of $27.6 \mathrm{kcal} / \mathrm{mol}$ at $298.15 \mathrm{~K}$ and $29.9 \mathrm{kcal} / \mathrm{mol}$ at $398.15 \mathrm{~K}$. In addition to overcoming the intrinsic energy barrier, the high temperature is required to remove water to promote the hydrogenation of $\alpha, \beta$-unsaturated ketone. This point is important for the selectivity of pro as we discussed on Page 23. 
Table S1. Calculated energy barriers with different DFT methods. ${ }^{a}$

\begin{tabular}{|l|l|l|l|}
\hline $\begin{array}{l}\text { Methods of geometry } \\
\text { optimization }\end{array}$ & $\mathbf{6 a} \rightarrow$ TS3a & $\mathbf{6 b} \rightarrow$ TS3b & ab6 $\rightarrow$ TS14 \\
\hline B3LYP/BS1 & 3.2 & 0.4 & 23.7 \\
\hline B3LYP-D3/BS1 & 3.0 & -1.0 & 24.7 \\
\hline$\omega$ B97XD/BS1 & 1.5 & 0.0 & 24.8 \\
\hline
\end{tabular}

${ }^{a}$ Solution-phase single-point energies are calculated at the level of M06/BS2/SMD. 
Table S2. Calculated thermodynamic corrections $\left(\Delta G_{c o r}\right)$, solution-phase single-point energies $\left(\Delta E_{\text {sol }}\right)$ and solution-phase Gibbs free energies referring to $1 \mathrm{M}$ and $298.15 \mathrm{~K}$ or $398.15 \mathrm{~K}\left(\Delta \mathrm{G}_{\mathrm{sol}}\right)$.

\begin{tabular}{|c|c|c|c|}
\hline species & $\Delta \mathbf{G}_{\text {cor }} /$ hartree & $\Delta \mathbf{E}_{\text {sol }} /$ hartree & $\Delta \mathbf{G}_{\text {sol }} /$ hartree \\
\hline \multicolumn{4}{|c|}{ B3LYP geometry optimization at $298.15 \mathrm{~K}$} \\
\hline 1 & 0.451809 & -2490.443378 & -2489.988541 \\
\hline${ }^{t} \mathrm{BuO}^{-}$ & 0.091495 & -233.023465 & -232.928942 \\
\hline 2 & 0.565015 & -2723.518581 & -2722.950538 \\
\hline TS1 & 0.560576 & -2723.516858 & -2722.953254 \\
\hline 3 & 0.562529 & -2723.520245 & -2722.954688 \\
\hline${ }^{t} \mathrm{BuOH}$ & 0.107237 & -233.584069 & -233.473804 \\
\hline 4 & 0.435066 & -2489.910636 & -2489.472542 \\
\hline TS2 & 0.435789 & -2489.899935 & -2489.461118 \\
\hline $\mathrm{Cl}^{-}$ & -0.015023 & -460.325791 & -460.337786 \\
\hline 5 & 0.433973 & -2029.572277 & -2029.135276 \\
\hline a1 & 0.128167 & -385.920211 & -385.789016 \\
\hline $6 \mathbf{a}$ & 0.584830 & -2415.515011 & -2414.927153 \\
\hline $6 b$ & 0.587574 & -2415.514830 & -2414.924228 \\
\hline TS3a & 0.586848 & -2415.511929 & -2414.922053 \\
\hline TS3b & 0.586413 & -2415.512995 & -2414.923554 \\
\hline 7 & 0.592953 & -2415.526968 & -2414.930987 \\
\hline 8 & 0.588166 & -2415.512289 & -2414.921095 \\
\hline TS4 & 0.588741 & -2415.506151 & -2414.914382 \\
\hline $\mathbf{a} 2$ & 0.105796 & -384.723339 & -384.614515 \\
\hline 9 & 0.458433 & -2030.770236 & -2030.308775 \\
\hline b1 & 0.145019 & -310.964058 & -310.816011 \\
\hline 10 & 0.603469 & -2340.553033 & -2339.946536 \\
\hline TS5 & 0.600455 & -2340.548326 & -2339.944843 \\
\hline 11 & 0.606111 & -2340.561919 & -2339.952780 \\
\hline 12 & 0.601216 & -2340.556340 & -2339.952096 \\
\hline TS6 & 0.603543 & -2340.550426 & -2339.943855 \\
\hline b2 & 0.121395 & -309.761491 & -309.637068 \\
\hline b1- & 0.129001 & -310.404572 & -310.272543 \\
\hline a1- & 0.113184 & -385.370011 & -385.253799 \\
\hline a1-CF3 & 0.101706 & -683.661063 & -683.556329 \\
\hline 13 & 0.607589 & -2416.709537 & -2416.098920 \\
\hline 13-CF3 & 0.581491 & -2714.452973 & -2713.868454 \\
\hline TS7 & 0.600211 & -2416.682598 & -2416.079359 \\
\hline TS7-CF3 & 0.573361 & -2714.433821 & -2713.857432 \\
\hline 14 & 0.601291 & -2416.689697 & -2416.085378 \\
\hline $\mathrm{H}_{2}$ & -0.001342 & -1.170085 & -1.168399 \\
\hline
\end{tabular}




\begin{tabular}{|c|c|c|c|}
\hline 15 & 0.625336 & -2341.753257 & -2341.124893 \\
\hline TS8 & 0.618806 & -2341.72401 & -2341.102176 \\
\hline 16 & 0.619802 & -2341.731593 & -2341.108763 \\
\hline TS9 & 0.451340 & -2030.729724 & -2030.275356 \\
\hline $\mathbf{a 3}$ & 0.215826 & -617.765184 & -617.546330 \\
\hline TS10 & 0.213461 & -617.758149 & -617.541660 \\
\hline a4 & 0.091039 & -384.178529 & -384.084462 \\
\hline TS11 & 0.237082 & -693.940366 & -693.700256 \\
\hline ab1 & 0.238556 & -693.948313 & -693.706729 \\
\hline ab2 & 0.365724 & -927.558825 & -927.190073 \\
\hline TS12 & 0.362992 & -927.558390 & -927.192370 \\
\hline ab3 & 0.366324 & -927.560742 & -927.191390 \\
\hline ab4 & 0.364881 & -927.548196 & -927.180287 \\
\hline TS13 & 0.363167 & -927.541152 & -927.174957 \\
\hline ab5 & 0.365758 & -927.564378 & -927.195592 \\
\hline ab6 & 0.36665 & -927.570160 & -927.200482 \\
\hline TS14 & 0.361264 & -927.527004 & -927.162712 \\
\hline TS14a & 0.245324 & -694.404642 & -694.159318 \\
\hline TS14b & 0.248965 & -694.410360 & -694.161395 \\
\hline ab7 & 0.358302 & -927.533254 & -927.171924 \\
\hline $\mathrm{H}_{2} \mathrm{O}$ & 0.003473 & -76.422333 & -76.415832 \\
\hline ab8 & 0.226568 & -618.051087 & -617.821491 \\
\hline ab9 & 0.252587 & -694.493228 & -694.240641 \\
\hline ab10 & 0.253699 & -694.498806 & -694.245107 \\
\hline ab11 & 0.254086 & -694.480755 & -694.226669 \\
\hline TS15 & 0.220783 & -768.906553 & -768.682742 \\
\hline aa1 & 0.350424 & -1002.522022 & -1002.168570 \\
\hline TS16 & 0.34804 & -1002.484605 & -1002.133537 \\
\hline aa2 & 0.21004 & -693.010250 & -692.797182 \\
\hline b3 & 0.106586 & -309.210356 & -309.100742 \\
\hline TS17 & 0.235328 & -693.940314 & -693.701958 \\
\hline ba1 & 0.363040 & -927.557862 & -927.191794 \\
\hline TS18 & 0.359212 & -927.521890 & -927.159650 \\
\hline ba2 & 0.224694 & -618.047515 & -617.819793 \\
\hline TS19 & 0.252214 & -618.978557 & -618.723315 \\
\hline bb1 & 0.382595 & -852.603238 & -852.217615 \\
\hline TS20 & 0.373537 & -852.560495 & -852.183930 \\
\hline bb2 & 0.241774 & -543.087635 & -542.842833 \\
\hline TS21 & 0.708742 & -2648.832283 & -2648.120513 \\
\hline 17 & 0.711959 & -2648.865712 & -2648.150725 \\
\hline
\end{tabular}




\begin{tabular}{|c|c|c|c|}
\hline 18 & 0.712063 & -2648.854121 & -2648.139030 \\
\hline TS22 & 0.707437 & -2648.852399 & -2648.141934 \\
\hline 19 & 0.705362 & -2648.846770 & -2648.138380 \\
\hline pro & 0.249536 & -619.277384 & -619.024820 \\
\hline TS23 & 0.710885 & -2648.821516 & -2648.107603 \\
\hline 20 & 0.706447 & -2648.836025 & -2648.126550 \\
\hline bpro1 & 0.248137 & -619.246048 & \begin{tabular}{|l|}
-618.994883 \\
\end{tabular} \\
\hline TS24 & 0.692218 & -2723.789985 & -2723.094739 \\
\hline TS25 & 0.691134 & -2723.807288 & -2723.113126 \\
\hline bpro2 & 0.232754 & -694.234429 & -693.998647 \\
\hline TS26 & 0.707251 & -2648.817635 & -2648.107356 \\
\hline TS27 & 0.705670 & -2648.844072 & -2648.135374 \\
\hline bpro3 & 0.248835 & -619.273939 & -619.022076 \\
\hline TS28 & 0.723307 & -2573.860873 & -2573.134538 \\
\hline TS29 & 0.722797 & -2573.877796 & -2573.151971 \\
\hline bpro4 & 0.265862 & -544.308042 & -544.039152 \\
\hline 21 & 0.726288 & -2650.061771 & -2649.332455 \\
\hline TS30 & 0.730718 & -2650.054755 & -2649.321009 \\
\hline 22 & 0.731861 & -2650.061876 & -2649.326987 \\
\hline 23 & 0.734730 & -2650.084350 & -2649.346592 \\
\hline TS31 & 0.728139 & -2650.060899 & -2649.329732 \\
\hline bpro5 & 0.271681 & -620.473627 & -620.198918 \\
\hline c1 & 0.205891 & -503.798349 & -503.589430 \\
\hline 24 & 0.684799 & -2534.591653 & -2533.903826 \\
\hline TS32 & 0.677046 & -2534.559674 & -2533.879600 \\
\hline 25 & 0.677554 & -2534.572124 & -2533.891542 \\
\hline d1 & 0.216264 & -465.734913 & -465.515621 \\
\hline 26 & 0.696643 & -2496.526676 & -2495.827005 \\
\hline TS33 & 0.687293 & -2496.496808 & -2495.806487 \\
\hline 27 & 0.689254 & -2496.504697 & -2495.812415 \\
\hline cd1 & 0.373043 & -890.702844 & -890.326773 \\
\hline TS34 & 0.856537 & -2921.472507 & -2920.612942 \\
\hline \multicolumn{4}{|c|}{ B3LYP geometry optimization at 398.15 K } \\
\hline ab8 & 0.206719 & -618.051087 & -617.841340 \\
\hline 9 & 0.421348 & -2030.770236 & -2030.345860 \\
\hline 9-D & 0.419043 & -2030.770236 & -2030.348165 \\
\hline TS21 & 0.659628 & -2648.832283 & -2648.169627 \\
\hline TS21-D & 0.657683 & -2648.832283 & -2648.171572 \\
\hline 1 & 0.413308 & -2490.443378 & -2490.027042 \\
\hline${ }^{t} \mathrm{BuO}^{-}$ & 0.078665 & -233.023465 & -232.941772 \\
\hline
\end{tabular}




\begin{tabular}{|c|c|c|c|}
\hline 3 & 0.517043 & -2723.520245 & -2723.000174 \\
\hline${ }^{t} \mathrm{BuOH}$ & 0.094254 & -233.584069 & -233.486787 \\
\hline TS2 & 0.397719 & -2489.899935 & -2489.499188 \\
\hline $\mathrm{Cl}^{-}$ & -0.020973 & -460.325791 & -460.343736 \\
\hline 7 & 0.548491 & -2415.526968 & -2414.975449 \\
\hline a1 & 0.112920 & -385.920211 & -385.804263 \\
\hline TS4 & 0.544880 & -2415.506151 & -2414.958243 \\
\hline $\mathbf{a 2}$ & 0.091065 & -384.723339 & -384.629246 \\
\hline b2 & 0.108006 & -309.761491 & -309.650457 \\
\hline 13 & 0.561265 & -2416.709537 & -2416.145244 \\
\hline TS7 & 0.554261 & -2416.682598 & -2416.125309 \\
\hline $\mathrm{H}_{2}$ & -0.006472 & -1.170085 & -1.173529 \\
\hline 5 & 0.395698 & -2029.572277 & -2029.173551 \\
\hline b1 & 0.131432 & -310.964058 & -310.829598 \\
\hline 12 & 0.555881 & -2340.556340 & -2339.997431 \\
\hline TS6 & 0.560745 & -2340.550426 & -2339.986653 \\
\hline b2 & 0.108006 & -309.761491 & -309.650457 \\
\hline a4 & 0.076370 & -384.178529 & -384.099131 \\
\hline TS11 & 0.216708 & -693.940366 & -693.720630 \\
\hline TS12 & 0.335873 & -927.558390 & -927.219489 \\
\hline TS13 & 0.336290 & -927.541152 & -927.201834 \\
\hline ab6 & 0.339150 & -927.570160 & -927.227982 \\
\hline TS14 & 0.333096 & -927.527004 & -927.190880 \\
\hline $\mathrm{H}_{2} \mathrm{O}$ & -0.003913 & -76.422333 & -76.423218 \\
\hline TS21 & 0.659628 & -2648.832283 & -2648.169627 \\
\hline pro & 0.229444 & -619.277384 & -619.044912 \\
\hline \multicolumn{4}{|c|}{ B3LYP-D3 geometry optimization at 298.15 K } \\
\hline $6 \mathbf{a}$ & 0.592693 & -2415.519167 & -2414.926474 \\
\hline TS3a & 0.591835 & -2415.513552 & -2414.921717 \\
\hline 6b & 0.594759 & -2415.517481 & -2414.922722 \\
\hline TS3b & 0.591438 & -2415.515810 & -2414.924372 \\
\hline ab6 & 0.369305 & -927.570897 & -927.201592 \\
\hline TS14 & 0.364352 & \begin{tabular}{|l|}
-927.526532 \\
\end{tabular} & -927.162180 \\
\hline \multicolumn{4}{|c|}{$\omega B 97 X D$ geometry optimization at $298.15 \mathrm{~K}$} \\
\hline $6 a$ & 0.601551 & -2415.521719 & -2414.920168 \\
\hline TS3a & 0.597878 & -2415.515696 & -2414.917818 \\
\hline 6b & 0.602626 & -2415.520165 & -2414.917539 \\
\hline TS3b & 0.600151 & -2415.517678 & -2414.917527 \\
\hline ab6 & 0.373299 & -927.572143 & -927.198844 \\
\hline TS14 & 0.368040 & -927.527411 & -927.159371 \\
\hline
\end{tabular}


Calculated Cartesian coordinates of all species (in angstrom) and imaginary frequency of all transition states (in $\mathbf{c m}^{-1}$ ). Unless mentioned otherwise, the following results were based on B3LYP geometry optimization.

\begin{tabular}{|c|c|c|c|}
\hline $\mathrm{Ru}$ & -0.01125900 & -0.02677400 & -0.01319500 \\
\hline $\mathrm{P}$ & 2.31169100 & 0.20336400 & 0.34907400 \\
\hline $\mathrm{P}$ & -2.35696000 & -0.08691400 & 0.29343800 \\
\hline $\mathrm{N}$ & -0.09353100 & 0.67575600 & 2.14826000 \\
\hline $\mathrm{C}$ & 2.37576700 & 0.50959200 & 2.21156100 \\
\hline $\mathrm{C}$ & 1.06332900 & 0.12017600 & 2.90071900 \\
\hline $\mathrm{C}$ & -2.36611300 & -0.44754800 & 2.12436300 \\
\hline $\mathrm{C}$ & -1.41815800 & 0.52151100 & 2.84030800 \\
\hline $\mathrm{C}$ & 3.60371800 & -2.25448600 & 0.93210100 \\
\hline $\mathrm{C}$ & 4.46003600 & -3.31924700 & 0.64880000 \\
\hline $\mathrm{C}$ & 5.19568200 & -3.33587100 & -0.53691900 \\
\hline $\mathrm{C}$ & 5.06919100 & -2.28168500 & -1.44335800 \\
\hline $\mathrm{C}$ & 4.22027900 & -1.21167500 & -1.15968300 \\
\hline $\mathrm{C}$ & 3.48516500 & -1.18104100 & 0.03647300 \\
\hline $\mathrm{C}$ & 2.63847700 & 2.51061300 & -1.24843700 \\
\hline $\mathrm{C}$ & 3.33036100 & 3.62092500 & -1.74151900 \\
\hline $\mathrm{C}$ & 4.62676600 & 3.89066400 & -1.30371900 \\
\hline $\mathrm{C}$ & 5.23753300 & 3.04280600 & -0.37459800 \\
\hline $\mathrm{C}$ & 4.55204600 & 1.93050200 & 0.11114900 \\
\hline $\mathrm{C}$ & 3.24077200 & 1.65549300 & -0.31649100 \\
\hline $\mathrm{C}$ & -2.97756100 & -2.65434800 & -0.63322600 \\
\hline $\mathrm{C}$ & -3.78852700 & -3.63985000 & -1.19664100 \\
\hline $\mathrm{C}$ & -5.09061700 & -3.33885200 & -1.60190400 \\
\hline $\mathrm{C}$ & -5.58300500 & -2.04324200 & -1.44013400 \\
\hline $\mathrm{C}$ & -4.78050800 & -1.05283800 & -0.87049400 \\
\hline $\mathrm{C}$ & -3.47295200 & -1.35056300 & -0.45515500 \\
\hline $\mathrm{C}$ & -4.34494300 & 1.85327500 & 0.97680000 \\
\hline $\mathrm{C}$ & -5.02751100 & 3.05984000 & 0.80713400 \\
\hline $\mathrm{C}$ & -4.67142600 & 3.92546800 & -0.22867400 \\
\hline $\mathrm{C}$ & -3.62861100 & 3.58081700 & -1.09102400 \\
\hline $\mathrm{C}$ & -2.93836300 & 2.38082000 & -0.91357700 \\
\hline $\mathrm{C}$ & -3.28859000 & 1.49902400 & 0.12094900 \\
\hline $\mathrm{H}$ & 3.21511100 & -0.02449400 & 2.66831900 \\
\hline $\mathrm{H}$ & 2.56679900 & 1.58192000 & 2.34177700 \\
\hline
\end{tabular}

$$
\begin{array}{rrr}
0.93754300 & -0.96433300 & 2.91863200 \\
1.06204600 & 0.49949700 & 3.93427800 \\
-3.36495800 & -0.40395800 & 2.57353500 \\
-1.99075800 & -1.47237900 & 2.20437200 \\
-1.88005800 & 1.51329600 & 2.89343000 \\
-1.24914800 & 0.18681100 & 3.87072400 \\
3.00768900 & -2.28102400 & 1.83698300 \\
4.54042200 & -4.14286500 & 1.35304500 \\
5.85848700 & -4.16858700 & -0.75685100 \\
5.63259400 & -2.28828600 & -2.37265800 \\
4.13859100 & -0.39475300 & -1.87058300 \\
1.62544900 & 2.30316700 & -1.57820400 \\
2.85260400 & 4.27333600 & -2.46761500 \\
5.16323400 & 4.75534400 & -1.68555500 \\
6.24934300 & 3.24560800 & -0.03341500 \\
\hline 5.04395400 & 1.26572700 & 0.81655300 \\
-1.96244000 & -2.89168500 & -0.32655100 \\
-3.39447900 & -4.64438200 & -1.32550500 \\
-5.71619900 & -4.10820300 & -2.04723900 \\
-6.59230500 & -1.79756400 & -1.76034300 \\
-5.17649200 & -0.04915200 & -0.75843300 \\
-4.64948800 & 1.18511300 & 1.77737900 \\
-5.83845000 & 3.32014300 & 1.48237300 \\
-5.20310600 & 4.86389500 & -0.36180800 \\
-3.34566000 & 4.24988100 & -1.89927800 \\
-2.11099300 & 2.12704000 & -1.56877700 \\
0.05493600 & -0.46267800 & -1.81201200 \\
0.09441800 & -0.70647300 & -2.94772700 \\
-0.11171500 & 1.52543500 & -0.42381500 \\
\hline .06840100 & 1.67445600 & 2.03361100 \\
\hline & -2.45598900 & 0.93892600 \\
\hline
\end{array}
$$$$
{ }^{t} \mathrm{BuO}^{-}
$$$$
\mathrm{C}
$$$$
\text { C }
$$$$
\mathrm{H}
$$$$
\mathrm{H}
$$$$
\mathrm{H}
$$$$
\text { C }
$$$$
\mathrm{H}
$$$$
\mathrm{H}
$$

$\begin{array}{rrr}-0.00001400 & -0.00001200 & 0.16182900 \\ -1.33011900 & -0.60914500 & -0.43743700 \\ -2.18208400 & -0.02618700 & -0.05888200 \\ -1.39200300 & -0.63751700 & -1.54173300 \\ -1.44538200 & -1.63500900 & -0.05887200 \\ 0.13752000 & 1.45647600 & -0.43737100 \\ -0.69307200 & 2.06935900 & -0.05856000 \\ 1.06853000 & 1.90266900 & -0.05906500\end{array}$




\begin{tabular}{|c|c|c|c|c|c|c|c|}
\hline $\mathrm{H}$ & 0.14359700 & 1.52424100 & -1.54166600 & $\mathrm{H}$ & -2.46952800 & 1.48731100 & -1.57892800 \\
\hline $\mathrm{C}$ & 1.19264500 & -0.84730900 & -0.43736600 & $\mathrm{H}$ & -1.14845900 & -0.96978300 & -2.83554600 \\
\hline $\mathrm{H}$ & 2.13866200 & -0.43407000 & -0.05884000 & $\mathrm{H}$ & -1.25284700 & 0.68977300 & -3.49817600 \\
\hline $\mathrm{H}$ & 1.11388300 & -1.87659800 & -0.05875600 & $\mathrm{H}$ & 2.23608200 & 1.84736400 & -1.38384300 \\
\hline $\mathrm{H}$ & 1.24814700 & -0.88676300 & -1.54166100 & $\mathrm{H}$ & 3.35579900 & 0.70846700 & -2.17385400 \\
\hline \multirow[t]{2}{*}{$\mathrm{O}$} & -0.00005900 & -0.00002400 & 1.48501300 & $\mathrm{H}$ & 1.19294500 & 0.97413900 & -3.44653000 \\
\hline & & & & $\mathrm{H}$ & 1.35331000 & -0.70039200 & -2.85481900 \\
\hline 2 & & & & $\mathrm{H}$ & -5.19427100 & -0.61961500 & -0.58672400 \\
\hline $\mathrm{Ru}$ & 0.06237500 & -0.69512500 & 0.12732000 & $\mathrm{H}$ & -6.76728100 & -2.52302000 & -0.67156600 \\
\hline $\mathrm{P}$ & -2.27617200 & -0.41813800 & -0.05941700 & $\mathrm{H}$ & -5.95483900 & -4.83312900 & -0.22059200 \\
\hline $\mathrm{P}$ & 2.35918100 & -0.20338100 & -0.07246500 & $\mathrm{H}$ & -3.54935700 & -5.21659400 & 0.28982400 \\
\hline $\mathrm{N}$ & -0.02483800 & 0.46651100 & -1.78293900 & $\mathrm{H}$ & -1.96467700 & -3.30845300 & 0.32461400 \\
\hline $\mathrm{C}$ & -2.45859500 & 0.40141900 & -1.73324300 & $\mathrm{H}$ & -2.00353600 & 2.48865000 & 0.30730600 \\
\hline $\mathrm{C}$ & -1.21794800 & 0.08987000 & -2.57497200 & $\mathrm{H}$ & -2.96526000 & 4.06766500 & 1.92817400 \\
\hline $\mathrm{C}$ & 2.39474500 & 0.79803500 & -1.65531300 & $\mathrm{H}$ & -4.53230700 & 3.29090800 & 3.70423900 \\
\hline $\mathrm{C}$ & 1.23134600 & 0.34262600 & -2.54344800 & $\mathrm{H}$ & -5.14207500 & 0.87881600 & 3.83380200 \\
\hline $\mathrm{C}$ & -4.82786600 & -1.62638500 & -0.40173000 & $\mathrm{H}$ & -4.22172400 & -0.71973500 & 2.19718400 \\
\hline $\mathrm{C}$ & -5.71844000 & -2.69861700 & -0.44277100 & $\mathrm{H}$ & 2.34263200 & -3.10375200 & -0.43583500 \\
\hline $\mathrm{C}$ & -5.26164700 & -3.99533900 & -0.18788600 & $\mathrm{H}$ & 4.12025600 & -4.80828300 & -0.72978500 \\
\hline $\mathrm{C}$ & -3.91451300 & -4.20982300 & 0.10269400 & $\mathrm{H}$ & 6.51365900 & -4.12435000 & -0.80324500 \\
\hline $\mathrm{C}$ & -3.01987200 & -3.13583300 & 0.14273000 & $\mathrm{H}$ & 7.11545000 & -1.72113800 & -0.55289700 \\
\hline $\mathrm{C}$ & -3.46883100 & -1.83199100 & -0.10542500 & $\mathrm{H}$ & 5.35133300 & -0.03194600 & -0.21494700 \\
\hline $\mathrm{C}$ & -2.70552800 & 2.12106700 & 1.06573200 & $\mathrm{H}$ & 4.08751200 & 2.28098300 & -0.16282200 \\
\hline $\mathrm{C}$ & -3.24401800 & 3.01807900 & 1.99015000 & $\mathrm{H}$ & 5.07346200 & 3.67770900 & 1.60658200 \\
\hline $\mathrm{C}$ & -4.12264100 & 2.58332900 & 2.98610600 & $\mathrm{H}$ & 4.68204600 & 3.12920800 & 4.00263000 \\
\hline $\mathrm{C}$ & -4.46614400 & 1.23312200 & 3.05833000 & $\mathrm{H}$ & 3.26826700 & 1.16751900 & 4.59939900 \\
\hline $\mathrm{C}$ & -3.94274100 & 0.32763800 & 2.13155200 & $\mathrm{H}$ & 2.25314400 & -0.21664600 & 2.82649500 \\
\hline $\mathrm{C}$ & -3.06859700 & 0.76217000 & 1.12416600 & $\mathrm{C}$ & 0.10283000 & -1.57430100 & 1.75743800 \\
\hline $\mathrm{C}$ & 3.38809500 & -2.80722000 & -0.43631900 & $\mathrm{O}$ & 0.13689700 & -2.10608100 & 2.79799400 \\
\hline $\mathrm{C}$ & 4.39386600 & -3.76185100 & -0.61874100 & $\mathrm{H}$ & 0.01240600 & 0.69060400 & 0.91659800 \\
\hline $\mathrm{C}$ & 5.73404000 & -3.37864000 & -0.66298900 & $\mathrm{H}$ & -0.17605200 & 1.50428500 & -1.55917000 \\
\hline $\mathrm{C}$ & 6.07250500 & -2.02928200 & -0.52474600 & $\mathrm{Cl}$ & 0.07755000 & -2.87886900 & -1.40039100 \\
\hline $\mathrm{C}$ & 5.07275900 & -1.07459400 & -0.34151600 & $\mathrm{C}$ & -0.62500500 & 4.18458900 & -1.92476000 \\
\hline $\mathrm{C}$ & 3.71930200 & -1.45176900 & -0.29868500 & $\mathrm{C}$ & -1.61881300 & 5.23925000 & -1.36321000 \\
\hline $\mathrm{C}$ & 3.90898000 & 2.01987700 & 0.87603300 & $\mathrm{H}$ & -1.45710400 & 5.35471400 & -0.28384500 \\
\hline $\mathrm{C}$ & 4.46987300 & 2.81510800 & 1.87888700 & $\mathrm{H}$ & -1.52036500 & 6.22897800 & -1.83527800 \\
\hline $\mathrm{C}$ & 4.24932600 & 2.50851400 & 3.22162100 & $\mathrm{H}$ & -2.64674300 & 4.88514800 & -1.51056100 \\
\hline $\mathrm{C}$ & 3.45788900 & 1.40706400 & 3.55600200 & $\mathrm{C}$ & 0.81994800 & 4.70609500 & -1.68027100 \\
\hline $\mathrm{C}$ & 2.89106600 & 0.61864900 & 2.55481700 & $\mathrm{H}$ & 1.01013400 & 4.74884600 & -0.60115600 \\
\hline $\mathrm{C}$ & 3.11538800 & 0.90895800 & 1.19947600 & $\mathrm{H}$ & 1.54661600 & 4.01018000 & -2.12129400 \\
\hline $\mathrm{H}$ & -3.37967300 & 0.07950800 & -2.23041200 & $\mathrm{H}$ & 1.00552400 & 5.70333500 & -2.10911200 \\
\hline
\end{tabular}




\begin{tabular}{|c|c|c|c|c|c|c|c|}
\hline $\mathrm{C}$ & -0.85083000 & 4.07748200 & -3.46060800 & $\mathrm{H}$ & -2.49897600 & 1.60559300 & -1.55903800 \\
\hline $\mathrm{H}$ & -0.15388600 & 3.34365900 & -3.88772200 & $\mathrm{H}$ & -1.18993700 & -0.85843800 & -2.83460900 \\
\hline $\mathrm{H}$ & -1.87034700 & 3.72234000 & -3.65767900 & $\mathrm{H}$ & -1.22457100 & 0.79823700 & -3.48254800 \\
\hline $\mathrm{H}$ & -0.70829700 & 5.03078500 & -3.99305300 & $\mathrm{H}$ & 2.34259500 & 1.75924700 & -1.38553700 \\
\hline \multirow[t]{2}{*}{$\mathrm{O}$} & -0.82635700 & 2.97678600 & -1.30657900 & $\mathrm{H}$ & 3.37369600 & 0.53588100 & -2.17322400 \\
\hline & & & & $\mathrm{H}$ & 1.22570900 & 0.94672700 & -3.43202100 \\
\hline \multicolumn{4}{|c|}{ TS1 } & $\mathrm{H}$ & 1.29263500 & -0.72854600 & -2.84460700 \\
\hline \multicolumn{4}{|c|}{ imaginary frequency $=-924.67 \mathrm{~cm}^{-1}$} & $\mathrm{H}$ & -5.21148200 & -0.43408100 & -0.72491200 \\
\hline $\mathrm{Ru}$ & 0.00978700 & -0.65334800 & 0.13317300 & $\mathrm{H}$ & -6.84068800 & -2.28866300 & -0.81062200 \\
\hline $\mathrm{P}$ & -2.30777300 & -0.30685200 & -0.06447000 & $\mathrm{H}$ & -6.12429900 & -4.60481600 & -0.24240100 \\
\hline $\mathrm{P}$ & 2.32003800 & -0.27191000 & -0.06009800 & $\mathrm{H}$ & -3.75623000 & -5.04381700 & 0.38508800 \\
\hline $\mathrm{N}$ & -0.01440300 & 0.54074400 & -1.75508300 & $\mathrm{H}$ & -2.11284600 & -3.18607100 & 0.41959400 \\
\hline $\mathrm{C}$ & -2.45923900 & 0.52745800 & -1.73578500 & $\mathrm{H}$ & -1.83486500 & 2.53668200 & 0.42178400 \\
\hline $\mathrm{C}$ & -1.20235100 & 0.20210900 & -2.55182700 & $\mathrm{H}$ & -2.76730400 & 4.16787800 & 2.01177700 \\
\hline $\mathrm{C}$ & 2.42355000 & 0.70135300 & -1.65260100 & $\mathrm{H}$ & -4.50726800 & 3.48605400 & 3.66148900 \\
\hline $\mathrm{C}$ & 1.21621500 & 0.31948800 & -2.51940500 & $\mathrm{H}$ & -5.31270200 & 1.12917500 & 3.69009200 \\
\hline $\mathrm{C}$ & -4.88562500 & -1.44391000 & -0.48750600 & $\mathrm{H}$ & -4.41827500 & -0.50581500 & 2.07640900 \\
\hline $\mathrm{C}$ & -5.80897600 & -2.48837300 & -0.52935900 & $\mathrm{H}$ & 2.15419700 & -3.15685400 & -0.49081000 \\
\hline $\mathrm{C}$ & -5.40624600 & -3.78821500 & -0.20863600 & $\mathrm{H}$ & 3.83984800 & -4.95661600 & -0.75169200 \\
\hline $\mathrm{C}$ & -4.08029300 & -4.03355400 & 0.14790800 & $\mathrm{H}$ & 6.27120100 & -4.41833100 & -0.69219400 \\
\hline $\mathrm{C}$ & -3.15338900 & -2.98750100 & 0.18801600 & $\mathrm{H}$ & 7.00104600 & -2.06265800 & -0.34573900 \\
\hline $\mathrm{C}$ & -3.54808000 & -1.67987600 & -0.12466000 & $\mathrm{H}$ & 5.32608600 & -0.27878200 & -0.04706300 \\
\hline $\mathrm{C}$ & -2.61446900 & 2.22239800 & 1.11773300 & $\mathrm{H}$ & 4.18975800 & 2.10555100 & -0.15680000 \\
\hline $\mathrm{C}$ & -3.13571700 & 3.14460600 & 2.02583300 & $\mathrm{H}$ & 5.22393100 & 3.47755800 & 1.60679000 \\
\hline $\mathrm{C}$ & -4.11008900 & 2.76258600 & 2.95246500 & $\mathrm{H}$ & 4.76099000 & 2.99487500 & 4.00453200 \\
\hline $\mathrm{C}$ & -4.56241700 & 1.44351900 & 2.96758700 & $\mathrm{H}$ & 3.22960300 & 1.12544000 & 4.60796600 \\
\hline $\mathrm{C}$ & -4.05367400 & 0.51633700 & 2.05327000 & $\mathrm{H}$ & 2.16838800 & -0.23105000 & 2.83944100 \\
\hline $\mathrm{C}$ & -3.08439900 & 0.89547200 & 1.11358000 & $\mathrm{C}$ & 0.01621400 & -1.56108400 & 1.75354100 \\
\hline $\mathrm{C}$ & 3.21470900 & -2.92305700 & -0.43715300 & $\mathrm{O}$ & 0.03090800 & -2.11053800 & 2.78687000 \\
\hline $\mathrm{C}$ & 4.16981800 & -3.93176800 & -0.59911300 & $\mathrm{H}$ & 0.02166100 & 0.72450100 & 0.93508500 \\
\hline $\mathrm{C}$ & 5.53123000 & -3.63027000 & -0.56863300 & $\mathrm{H}$ & -0.12760200 & 1.84935900 & -1.48595800 \\
\hline $\mathrm{C}$ & 5.94145300 & -2.30793400 & -0.37624200 & $\mathrm{Cl}$ & -0.09854900 & -2.85544600 & -1.39211100 \\
\hline $\mathrm{C}$ & 4.99213600 & -1.29903200 & -0.21424700 & $\mathrm{C}$ & -0.20099600 & 4.05562700 & -1.99067800 \\
\hline $\mathrm{C}$ & 3.61800400 & -1.59348400 & -0.24598900 & $\mathrm{C}$ & -0.87855500 & 5.26851500 & -1.31598000 \\
\hline $\mathrm{C}$ & 3.97848000 & 1.87555600 & 0.88339000 & $\mathrm{H}$ & -0.42361200 & 5.44353800 & -0.33409000 \\
\hline $\mathrm{C}$ & 4.56667900 & 2.65595500 & 1.88250900 & $\mathrm{H}$ & -0.78817300 & 6.18729700 & -1.91283500 \\
\hline $\mathrm{C}$ & 4.30670300 & 2.38615600 & 3.22615800 & $\mathrm{H}$ & -1.94340400 & 5.05859400 & -1.15982800 \\
\hline $\mathrm{C}$ & 3.44928300 & 1.33629300 & 3.56414700 & $\mathrm{C}$ & 1.29739200 & 4.38329300 & -2.20820100 \\
\hline $\mathrm{C}$ & 2.85618100 & 0.56308800 & 2.56613800 & $\mathrm{H}$ & 1.78617600 & 4.52271400 & -1.23694900 \\
\hline $\mathrm{C}$ & 3.11824100 & 0.81622000 & 1.21007900 & $\mathrm{H}$ & 1.79937100 & 3.55628900 & -2.72548300 \\
\hline $\mathrm{H}$ & -3.37395100 & 0.21407000 & -2.25045300 & $\mathrm{H}$ & 1.44609700 & 5.29304700 & -2.80785700 \\
\hline
\end{tabular}




\begin{tabular}{|c|c|c|c|c|c|c|c|}
\hline $\mathrm{C}$ & -0.86479900 & 3.81553500 & -3.36831400 & $\mathrm{H}$ & -1.23625200 & -0.81866700 & -2.85106900 \\
\hline $\mathrm{H}$ & -0.39319400 & 2.96862300 & -3.88133300 & $\mathrm{H}$ & -1.20652700 & 0.84259000 & -3.47277800 \\
\hline $\mathrm{H}$ & -1.92813500 & 3.58127400 & -3.23840300 & $\mathrm{H}$ & 2.42171400 & 1.67827900 & -1.46244100 \\
\hline $\mathrm{H}$ & -0.78464400 & 4.69320900 & -4.02551100 & $\mathrm{H}$ & 3.37946400 & 0.38181500 & -2.22399800 \\
\hline \multirow[t]{2}{*}{$\mathrm{O}$} & -0.35729700 & 2.96531300 & -1.13378000 & $\mathrm{H}$ & 1.22330600 & 0.86902200 & -3.45891300 \\
\hline & & & & $\mathrm{H}$ & 1.26151500 & -0.79790200 & -2.85546600 \\
\hline 3 & & & & $\mathrm{H}$ & -5.19782400 & -0.37739000 & -0.81219700 \\
\hline $\mathrm{Ru}$ & 0.00123000 & -0.65403100 & 0.12846100 & $\mathrm{H}$ & -6.84640200 & -2.21187900 & -0.93670400 \\
\hline $\mathrm{P}$ & -2.30856200 & -0.28335900 & -0.07259300 & $\mathrm{H}$ & -6.17224800 & -4.53603900 & -0.34911600 \\
\hline $\mathrm{P}$ & 2.31417500 & -0.31382800 & -0.07898400 & $\mathrm{H}$ & -3.82561400 & -5.00256600 & 0.33671600 \\
\hline $\mathrm{N}$ & 0.00222000 & 0.52958400 & -1.75424700 & $\mathrm{H}$ & -2.16086800 & -3.16487600 & 0.40827000 \\
\hline $\mathrm{C}$ & -2.44311900 & 0.58932800 & -1.72463600 & $\mathrm{H}$ & -1.83190400 & 2.54769600 & 0.51317200 \\
\hline $\mathrm{C}$ & -1.19304900 & 0.23845000 & -2.54236100 & $\mathrm{H}$ & -2.78022400 & 4.13759400 & 2.12973600 \\
\hline $\mathrm{C}$ & 2.44467100 & 0.61009800 & -1.69925200 & $\mathrm{H}$ & -4.54993200 & 3.41695700 & 3.73053800 \\
\hline $\mathrm{C}$ & 1.20497400 & 0.25628600 & -2.53312800 & $\mathrm{H}$ & -5.36415600 & 1.06321700 & 3.67724300 \\
\hline $\mathrm{C}$ & -4.88937800 & -1.39075700 & -0.56655100 & $\mathrm{H}$ & -4.45135200 & -0.52751800 & 2.03146600 \\
\hline $\mathrm{C}$ & -5.82433200 & -2.42384200 & -0.63010500 & $\mathrm{H}$ & 2.08927100 & -3.19903700 & -0.46323500 \\
\hline $\mathrm{C}$ & -5.44543200 & -3.72804900 & -0.29837900 & $\mathrm{H}$ & 3.73407600 & -5.04331800 & -0.66384800 \\
\hline $\mathrm{C}$ & -4.13166500 & -3.98872900 & 0.09093100 & $\mathrm{H}$ & 6.17701900 & -4.56431200 & -0.56847400 \\
\hline $\mathrm{C}$ & -3.19316900 & -2.95405800 & 0.15222700 & $\mathrm{H}$ & 6.95838000 & -2.22133400 & -0.24774400 \\
\hline $\mathrm{C}$ & -3.56392900 & -1.64207800 & -0.17146800 & $\mathrm{H}$ & 5.32349600 & -0.39259300 & -0.01035400 \\
\hline $\mathrm{C}$ & -2.62412800 & 2.21683300 & 1.18224000 & $\mathrm{H}$ & 4.27113100 & 1.99070100 & -0.22895200 \\
\hline $\mathrm{C}$ & -3.15428800 & 3.11645700 & 2.10730900 & $\mathrm{H}$ & 5.32358800 & 3.39304900 & 1.50084100 \\
\hline $\mathrm{C}$ & -4.14478300 & 2.71265200 & 3.00700600 & $\mathrm{H}$ & 4.80359900 & 3.01786600 & 3.90621100 \\
\hline $\mathrm{C}$ & -4.60155200 & 1.39551600 & 2.97600900 & $\mathrm{H}$ & 3.19776200 & 1.22618100 & 4.55082900 \\
\hline $\mathrm{C}$ & -4.08208300 & 0.49295200 & 2.04332400 & $\mathrm{H}$ & 2.11816400 & -0.15842000 & 2.81371100 \\
\hline $\mathrm{C}$ & -3.09618000 & 0.89224300 & 1.12928600 & $\mathrm{C}$ & -0.00167800 & -1.56208800 & 1.75344300 \\
\hline $\mathrm{C}$ & 3.15429500 & -2.99017300 & -0.39426700 & $\mathrm{O}$ & 0.00821000 & -2.11389500 & 2.78638800 \\
\hline $\mathrm{C}$ & 4.08685000 & -4.02445300 & -0.52226500 & $\mathrm{H}$ & 0.03299500 & 0.72920900 & 0.92266900 \\
\hline $\mathrm{C}$ & 5.45468900 & -3.75629000 & -0.47174400 & $\mathrm{H}$ & -0.08276100 & 2.19448200 & -1.35955000 \\
\hline $\mathrm{C}$ & 5.89372100 & -2.44133600 & -0.29377000 & $\mathrm{Cl}$ & -0.14350700 & -2.87196200 & -1.38388700 \\
\hline $\mathrm{C}$ & 4.96676300 & -1.40681600 & -0.16609500 & $\mathrm{C}$ & -0.08996700 & 4.17673000 & -1.94833900 \\
\hline $\mathrm{C}$ & 3.58646500 & -1.66746300 & -0.21777800 & $\mathrm{C}$ & -0.33882800 & 5.48704500 & -1.18384000 \\
\hline $\mathrm{C}$ & 4.03329200 & 1.80938700 & 0.81535200 & $\mathrm{H}$ & 0.38470500 & 5.58519200 & -0.36700900 \\
\hline $\mathrm{C}$ & 4.63232700 & 2.60627000 & 1.79488900 & $\mathrm{H}$ & -0.25070800 & 6.36264400 & -1.83995900 \\
\hline $\mathrm{C}$ & 4.34077000 & 2.39657600 & 3.14285200 & $\mathrm{H}$ & -1.34326000 & 5.47967900 & -0.74535100 \\
\hline $\mathrm{C}$ & 3.44153500 & 1.39025000 & 3.50390500 & $\mathrm{C}$ & 1.32998700 & 4.19324600 & -2.55107600 \\
\hline $\mathrm{C}$ & 2.83795300 & 0.60100100 & 2.52475100 & $\mathrm{H}$ & 2.07331700 & 4.28243500 & -1.75069800 \\
\hline $\mathrm{C}$ & 3.13062400 & 0.79337600 & 1.16498200 & $\mathrm{H}$ & 1.52771200 & 3.26543600 & -3.09998300 \\
\hline $\mathrm{H}$ & -3.36684100 & 0.31114300 & -2.24389600 & $\mathrm{H}$ & 1.46640900 & 5.03255300 & -3.24660000 \\
\hline $\mathrm{H}$ & -2.46432100 & 1.66458800 & -1.52594400 & $\mathrm{C}$ & -1.13193500 & 4.02916100 & -3.07631900 \\
\hline
\end{tabular}




\begin{tabular}{|c|c|c|c|c|c|c|c|}
\hline $\mathrm{H}$ & -0.98017600 & 3.09172300 & -3.62284300 & $\mathrm{C}$ & 2.82350100 & 2.23299600 & -1.21131400 \\
\hline $\mathrm{H}$ & -2.14367300 & 4.01628900 & -2.65506000 & $\mathrm{C}$ & 3.08633200 & 1.66567200 & 0.04635100 \\
\hline $\mathrm{H}$ & -1.06947500 & 4.85590500 & -3.79702000 & $\mathrm{C}$ & -3.24272900 & -2.26566700 & -0.88465200 \\
\hline \multirow[t]{2}{*}{$\mathrm{O}$} & -0.21994300 & 3.14697800 & -0.98861300 & $\mathrm{C}$ & -4.21524500 & -3.15150000 & -1.36009500 \\
\hline & & & & $\mathrm{C}$ & -5.56889300 & -2.91028000 & -1.12661400 \\
\hline${ }^{t} \mathrm{BuOH}$ & & & & $\mathrm{C}$ & -5.95408400 & -1.77416500 & -0.40878100 \\
\hline $\mathrm{C}$ & -0.00530400 & -0.00000100 & 0.01473300 & $\mathrm{C}$ & -4.98688800 & -0.88853000 & 0.06576300 \\
\hline $\mathrm{C}$ & 0.69065300 & -1.26539900 & -0.51025300 & $\mathrm{C}$ & -3.62037000 & -1.12356300 & -0.16445800 \\
\hline $\mathrm{H}$ & 0.21097000 & -2.15980600 & -0.09900200 & $\mathrm{C}$ & -3.93655700 & 2.35746900 & 0.92225100 \\
\hline $\mathrm{H}$ & 0.65340200 & -1.32193700 & -1.60450700 & $\mathrm{C}$ & -4.51356600 & 3.57578600 & 0.55214100 \\
\hline $\mathrm{H}$ & 1.74821200 & -1.27905900 & -0.21399900 & $\mathrm{C}$ & -4.25279600 & 4.12125700 & -0.70495500 \\
\hline $\mathrm{C}$ & -1.49047000 & -0.00029800 & -0.35682900 & $\mathrm{C}$ & -3.40533600 & 3.44411000 & -1.58571900 \\
\hline $\mathrm{H}$ & -1.98392900 & -0.88689800 & 0.05496100 & $\mathrm{C}$ & -2.82335300 & 2.23293000 & -1.21146200 \\
\hline $\mathrm{H}$ & -1.98429500 & 0.88607600 & 0.05501200 & $\mathrm{C}$ & -3.08631800 & 1.66568800 & 0.04621400 \\
\hline $\mathrm{H}$ & -1.62219500 & -0.00029400 & -1.44423000 & $\mathrm{H}$ & 3.36669700 & -0.53205700 & 2.64378100 \\
\hline $\mathrm{C}$ & 0.69012900 & 1.26570900 & -0.51019900 & $\mathrm{H}$ & 2.32242100 & 0.91683800 & 2.72719500 \\
\hline $\mathrm{H}$ & 0.21007700 & 2.15989500 & -0.09889900 & $\mathrm{H}$ & 1.33866100 & -1.97655300 & 2.48407400 \\
\hline $\mathrm{H}$ & 1.74768300 & 1.27979300 & -0.21395000 & $\mathrm{H}$ & 1.17320000 & -0.90321100 & 3.88848300 \\
\hline $\mathrm{H}$ & 0.65284400 & 1.32228600 & -1.60445000 & $\mathrm{H}$ & -2.32233400 & 0.91692800 & 2.72719000 \\
\hline $\mathrm{O}$ & 0.01412100 & -0.00002400 & 1.45181100 & $\mathrm{H}$ & -3.36673200 & -0.53188600 & 2.64383800 \\
\hline \multirow[t]{2}{*}{$\mathrm{H}$} & 0.94422300 & 0.00007100 & 1.72987200 & $\mathrm{H}$ & -1.17323800 & -0.90319100 & 3.88848600 \\
\hline & & & & $\mathrm{H}$ & -1.33875200 & -1.97651100 & 2.48407100 \\
\hline 4 & & & & $\mathrm{H}$ & 5.30171200 & -0.00152500 & 0.60874400 \\
\hline $\mathrm{Ru}$ & -0.00002600 & -0.18311100 & 0.05974100 & $\mathrm{H}$ & 7.00745400 & -1.57607800 & -0.22187800 \\
\hline $\mathrm{P}$ & 2.30211300 & 0.03453800 & 0.46167700 & $\mathrm{H}$ & 6.32220600 & -3.60081200 & -1.50045900 \\
\hline $\mathrm{P}$ & -2.30218500 & 0.03455200 & 0.46167400 & $\mathrm{H}$ & 3.90462600 & -4.03451700 & -1.91389000 \\
\hline $\mathrm{N}$ & -0.00001400 & -0.31518300 & 2.23430600 & $\mathrm{H}$ & 2.18672500 & -2.47518200 & -1.03496200 \\
\hline $\mathrm{C}$ & 2.41335000 & -0.09687600 & 2.32192400 & $\mathrm{H}$ & 4.14968800 & 1.95051200 & 1.90691600 \\
\hline $\mathrm{C}$ & 1.19630300 & -0.91672300 & 2.77815200 & $\mathrm{H}$ & 5.16493400 & 4.09749500 & 1.25049300 \\
\hline $\mathrm{C}$ & -2.41336100 & -0.09678400 & 2.32194500 & $\mathrm{H}$ & 4.69985400 & 5.06986000 & -0.99357200 \\
\hline $\mathrm{C}$ & -1.19634900 & -0.91668600 & 2.77815500 & $\mathrm{H}$ & 3.18685900 & 3.86548400 & -2.56391600 \\
\hline $\mathrm{C}$ & 4.98667600 & -0.88933200 & 0.06684700 & $\mathrm{H}$ & 2.14337300 & 1.72910900 & -1.89126200 \\
\hline $\mathrm{C}$ & 5.95386900 & -1.77482700 & -0.40795900 & $\mathrm{H}$ & -2.18675600 & -2.47659400 & -1.03248000 \\
\hline $\mathrm{C}$ & 5.56874500 & -2.91001400 & -1.12729600 & $\mathrm{H}$ & -3.90464800 & -4.03624900 & -1.91085400 \\
\hline $\mathrm{C}$ & 4.21517100 & -3.15046500 & -1.36198700 & $\mathrm{H}$ & -6.32236000 & -3.60118400 & -1.49957200 \\
\hline $\mathrm{C}$ & 3.24266400 & -2.26477400 & -0.88625800 & $\mathrm{H}$ & -7.00772400 & -1.57479800 & -0.22367200 \\
\hline $\mathrm{C}$ & 3.62023100 & -1.12357600 & -0.16460000 & $\mathrm{H}$ & -5.30196000 & -0.00001800 & 0.60648200 \\
\hline $\mathrm{C}$ & 3.93646800 & 2.35739500 & 0.92253100 & $\mathrm{H}$ & -4.14997400 & 1.95060200 & 1.90659600 \\
\hline $\mathrm{C}$ & 4.51357200 & 3.57570100 & 0.55254500 & $\mathrm{H}$ & -5.16501200 & 4.09762200 & 1.24997800 \\
\hline $\mathrm{C}$ & 4.25299000 & 4.12122700 & -0.70456800 & $\mathrm{H}$ & -4.69958800 & 5.06989500 & -0.99405400 \\
\hline $\mathrm{C}$ & 3.40559600 & 3.44415900 & -1.58545600 & $\mathrm{H}$ & -3.18646700 & 3.86538200 & -2.56417100 \\
\hline
\end{tabular}




\begin{tabular}{|c|c|c|c|c|c|c|c|}
\hline $\mathrm{H}$ & -2.14317100 & 1.72898400 & -1.89131400 & $\mathrm{H}$ & 2.34578500 & 0.90084800 & 2.71219500 \\
\hline $\mathrm{C}$ & -0.00006200 & -0.01822600 & -1.79942000 & $\mathrm{H}$ & 1.36584800 & -1.99693400 & 2.44353000 \\
\hline $\mathrm{O}$ & -0.00009800 & 0.09643400 & -2.96748300 & $\mathrm{H}$ & 1.13231600 & -0.88141700 & 3.80218100 \\
\hline $\mathrm{H}$ & -0.00006400 & 1.40759700 & 0.20509200 & $\mathrm{H}$ & -2.34584300 & 0.90073100 & 2.71223500 \\
\hline \multirow[t]{2}{*}{$\mathrm{Cl}$} & 0.00036000 & -2.89088300 & 0.03294900 & $\mathrm{H}$ & -3.36878500 & -0.55586600 & 2.57120400 \\
\hline & & & & $\mathrm{H}$ & -1.13228500 & -0.88149000 & 3.80217000 \\
\hline \multicolumn{4}{|l|}{ TS2 } & $\mathrm{H}$ & -1.36576600 & -1.99699700 & 2.44350200 \\
\hline \multicolumn{4}{|c|}{ imaginary frequency $=-52.58 \mathrm{~cm}^{-1}$} & $\mathrm{H}$ & 5.20307200 & 0.22569700 & -0.52028300 \\
\hline $\mathrm{Ru}$ & 0.00000300 & 0.04409000 & 0.05092800 & $\mathrm{H}$ & 6.70303600 & -1.50919300 & -1.42250800 \\
\hline $\mathrm{P}$ & 2.32138400 & 0.13302300 & 0.41566700 & $\mathrm{H}$ & 5.88376600 & -3.84160300 & -1.72082000 \\
\hline $\mathrm{P}$ & -2.32137700 & 0.13299200 & 0.41567600 & $\mathrm{H}$ & 3.52641700 & -4.40524200 & -1.10563500 \\
\hline $\mathrm{N}$ & 0.00000700 & -0.40626300 & 2.08234600 & $\mathrm{H}$ & 2.01791400 & -2.69359300 & -0.22329900 \\
\hline $\mathrm{C}$ & 2.42023800 & -0.09823600 & 2.26748300 & $\mathrm{H}$ & 4.43244100 & 1.72285100 & 1.85363000 \\
\hline $\mathrm{C}$ & 1.20505000 & -0.93395100 & 2.69687100 & $\mathrm{H}$ & 5.53104000 & 3.87545600 & 1.35474800 \\
\hline $\mathrm{C}$ & -2.42023800 & -0.09834000 & 2.26748400 & $\mathrm{H}$ & 4.92990700 & 5.13933700 & -0.70336300 \\
\hline $\mathrm{C}$ & -1.20501500 & -0.93401200 & 2.69685900 & $\mathrm{H}$ & 3.20344000 & 4.22652800 & -2.24797300 \\
\hline $\mathrm{C}$ & 4.83089600 & -0.78693300 & -0.64167700 & $\mathrm{H}$ & 2.07609100 & 2.08451100 & -1.72466200 \\
\hline $\mathrm{C}$ & 5.68128900 & -1.76919000 & -1.15289400 & $\mathrm{H}$ & -2.01792100 & -2.69362100 & -0.22331400 \\
\hline $\mathrm{C}$ & 5.22032800 & -3.07713000 & -1.32110700 & $\mathrm{H}$ & -3.52642200 & -4.40524100 & -1.10572100 \\
\hline $\mathrm{C}$ & 3.90451700 & -3.39395000 & -0.97988800 & $\mathrm{H}$ & -5.88373900 & -3.84155700 & -1.72098100 \\
\hline $\mathrm{C}$ & 3.04377800 & -2.41504700 & -0.47744200 & $\mathrm{H}$ & -6.70298400 & -1.50913800 & -1.42267500 \\
\hline $\mathrm{C}$ & 3.50629200 & -1.10158400 & -0.29508000 & $\mathrm{H}$ & -5.20302300 & 0.22571900 & -0.52037700 \\
\hline $\mathrm{C}$ & 4.15487500 & 2.26613400 & 0.95464000 & $\mathrm{H}$ & -4.43248400 & 1.72272000 & 1.85367600 \\
\hline $\mathrm{C}$ & 4.78089400 & 3.48288600 & 0.67185300 & $\mathrm{H}$ & -5.53111800 & 3.87532400 & 1.35487000 \\
\hline $\mathrm{C}$ & 4.44442500 & 4.19145400 & -0.48269800 & $\mathrm{H}$ & -4.92997800 & 5.13930700 & -0.70317700 \\
\hline $\mathrm{C}$ & 3.47646400 & 3.67847200 & -1.34936000 & $\mathrm{H}$ & -3.20346800 & 4.22659900 & -2.24779900 \\
\hline $\mathrm{C}$ & 2.84539800 & 2.46754500 & -1.06116300 & $\mathrm{H}$ & -2.07608200 & 2.08458300 & -1.72456100 \\
\hline $\mathrm{C}$ & 3.17887700 & 1.74150500 & 0.09301100 & $\mathrm{C}$ & 0.00000100 & 0.18438400 & -1.81415500 \\
\hline $\mathrm{C}$ & -3.04377400 & -2.41506000 & -0.47748800 & $\mathrm{O}$ & 0.00000000 & 0.23527000 & -2.98611400 \\
\hline $\mathrm{C}$ & -3.90451000 & -3.39394500 & -0.97997500 & $\mathrm{H}$ & -0.00000300 & 1.61674800 & 0.10279400 \\
\hline $\mathrm{C}$ & -5.22030400 & -3.07710000 & -1.32123800 & $\mathrm{Cl}$ & 0.00000000 & -3.72330900 & 0.48084300 \\
\hline $\mathrm{C}$ & -5.68125000 & -1.76915400 & -1.15302800 & & & & \\
\hline $\mathrm{C}$ & -4.83086000 & -0.78691500 & -0.64177100 & 5 & & & \\
\hline $\mathrm{C}$ & -3.50627300 & -1.10159200 & -0.29513000 & $\mathrm{Ru}$ & 0.00001300 & -0.25665000 & 0.15935400 \\
\hline $\mathrm{C}$ & -4.15491400 & 2.26604800 & 0.95471400 & $\mathrm{P}$ & 2.32782700 & -0.04597500 & 0.43225000 \\
\hline $\mathrm{C}$ & -4.78095300 & 3.48279900 & 0.67197000 & $\mathrm{P}$ & -2.32763300 & -0.04640900 & 0.43264100 \\
\hline $\mathrm{C}$ & -4.44448000 & 4.19142400 & -0.48254500 & $\mathrm{~N}$ & -0.00001700 & 0.24665400 & 2.13527600 \\
\hline $\mathrm{C}$ & -3.47649500 & 3.67849900 & -1.34921400 & $\mathrm{C}$ & 2.44162400 & -0.02854500 & 2.29047000 \\
\hline $\mathrm{C}$ & -2.84540900 & 2.46757300 & -1.06105800 & $\mathrm{C}$ & 1.20277100 & 0.68479300 & 2.83823500 \\
\hline $\mathrm{C}$ & -3.17889200 & 1.74147600 & 0.09307900 & $\mathrm{C}$ & -2.44154100 & -0.02916700 & 2.29070200 \\
\hline $\mathrm{H}$ & 3.36880400 & -0.55570200 & 2.57123300 & $\mathrm{C}$ & -1.20276500 & 0.68413600 & 2.83871300 \\
\hline
\end{tabular}




\begin{tabular}{|c|c|c|c|c|c|c|c|}
\hline $\mathrm{C}$ & 3.05697700 & -2.69493500 & -0.07878700 & $\mathrm{H}$ & 4.39431900 & 1.85763400 & 1.55827100 \\
\hline $\mathrm{C}$ & 3.91571000 & -3.74237600 & -0.41206800 & $\mathrm{H}$ & -2.01790000 & -2.90162300 & 0.13903100 \\
\hline $\mathrm{C}$ & 5.24309700 & -3.47719400 & -0.75572900 & $\mathrm{H}$ & -3.54805400 & -4.75856500 & -0.44265600 \\
\hline $\mathrm{C}$ & 5.70639900 & -2.16050200 & -0.76315000 & $\mathrm{H}$ & -5.92110600 & -4.28346700 & -1.02223900 \\
\hline $\mathrm{C}$ & 4.85000800 & -1.11140600 & -0.42303700 & $\mathrm{H}$ & -6.74858100 & -1.93851800 & -1.00674600 \\
\hline $\mathrm{C}$ & 3.51641200 & -1.36781300 & -0.07148300 & $\mathrm{H}$ & -5.22880900 & -0.08772500 & -0.40954900 \\
\hline $\mathrm{C}$ & 2.74498000 & 2.01170000 & -1.41787400 & $\mathrm{H}$ & -4.36960700 & 1.87916100 & 1.56916800 \\
\hline $\mathrm{C}$ & 3.32679300 & 3.17327800 & -1.92545700 & $\mathrm{H}$ & -5.36630400 & 3.94180200 & 0.67687900 \\
\hline $\mathrm{C}$ & 4.28097200 & 3.86583900 & -1.17674700 & $\mathrm{H}$ & -4.71903800 & 4.77666300 & -1.57489400 \\
\hline $\mathrm{C}$ & 4.65511000 & 3.38618000 & 0.07903500 & $\mathrm{H}$ & -3.04169100 & 3.52210800 & -2.91918300 \\
\hline $\mathrm{C}$ & 4.08064300 & 2.21673400 & 0.58301600 & $\mathrm{H}$ & -2.00327300 & 1.46975300 & -2.00524300 \\
\hline $\mathrm{C}$ & 3.11669700 & 1.51473900 & -0.15780700 & $\mathrm{C}$ & 0.00058800 & -1.30343000 & -1.40353900 \\
\hline $\mathrm{C}$ & -3.05857900 & -2.69236100 & -0.09435100 & $\mathrm{O}$ & 0.00089000 & -1.99005300 & -2.34638500 \\
\hline $\mathrm{C}$ & -3.92020800 & -3.73762000 & -0.42732200 & $\mathrm{H}$ & -0.00118300 & 0.94404300 & -0.86865600 \\
\hline $\mathrm{C}$ & -5.25130900 & -3.47051900 & -0.75452400 & & & & \\
\hline $\mathrm{C}$ & -5.71562500 & -2.15411500 & -0.74593400 & a1 & & & \\
\hline $\mathrm{C}$ & -4.85629400 & -1.10734300 & -0.40637400 & $\mathrm{C}$ & -2.06807400 & -1.09649100 & -0.21434300 \\
\hline $\mathrm{C}$ & -3.51877400 & -1.36577500 & -0.07130600 & $\mathrm{C}$ & -0.68728900 & -1.20545800 & -0.38235600 \\
\hline $\mathrm{C}$ & -4.06475800 & 2.22878900 & 0.58772600 & $\mathrm{C}$ & 0.14718100 & -0.09768300 & -0.18883100 \\
\hline $\mathrm{C}$ & -4.63706600 & 3.39846700 & 0.08161500 & $\mathrm{C}$ & -0.42651700 & 1.12835300 & 0.16659900 \\
\hline $\mathrm{C}$ & -4.27392000 & 3.86653200 & -1.18166800 & $\mathrm{C}$ & -1.80866200 & 1.24012300 & 0.33020000 \\
\hline $\mathrm{C}$ & -3.33255600 & 3.16231900 & -1.93583700 & $\mathrm{C}$ & -2.63363400 & 0.12931900 & 0.14370400 \\
\hline $\mathrm{C}$ & -2.75269500 & 2.00074700 & -1.42623600 & $\mathrm{H}$ & -2.70185800 & -1.96562200 & -0.37089700 \\
\hline $\mathrm{C}$ & -3.11388900 & 1.51511500 & -0.15862800 & $\mathrm{H}$ & -0.25365300 & -2.16082400 & -0.67241600 \\
\hline $\mathrm{H}$ & 2.41667200 & -1.08381500 & 2.58558200 & $\mathrm{H}$ & 0.21633200 & 1.99196300 & 0.29847200 \\
\hline $\mathrm{H}$ & 3.37318100 & 0.40220200 & 2.67269800 & $\mathrm{H}$ & -2.24196600 & 2.19962900 & 0.60128800 \\
\hline $\mathrm{H}$ & 1.11575700 & 0.46484700 & 3.91694500 & $\mathrm{H}$ & -3.70944300 & 0.21876100 & 0.26978800 \\
\hline $\mathrm{H}$ & 1.34310200 & 1.77991200 & 2.76186200 & $\mathrm{C}$ & 1.65145800 & -0.25186300 & -0.32169700 \\
\hline $\mathrm{H}$ & -3.37338200 & 0.40076400 & 2.67319800 & $\mathrm{H}$ & 1.85258000 & -0.97543500 & -1.12970600 \\
\hline $\mathrm{H}$ & -2.41622000 & -1.08461600 & 2.58517100 & $\mathrm{O}$ & 2.20301300 & 1.01865500 & -0.66504000 \\
\hline $\mathrm{H}$ & -1.34329300 & 1.77927800 & 2.76293300 & $\mathrm{H}$ & 3.16811400 & 0.93812700 & -0.62253800 \\
\hline $\mathrm{H}$ & -1.11549000 & 0.46365200 & 3.91729300 & $\mathrm{C}$ & 2.27988400 & -0.78601900 & 0.97438400 \\
\hline $\mathrm{H}$ & 2.01889900 & -2.90299100 & 0.16711100 & $\mathrm{H}$ & 2.09605700 & -0.08648500 & 1.79672100 \\
\hline $\mathrm{H}$ & 3.54415100 & -4.76365200 & -0.41485600 & $\mathrm{H}$ & 1.85848800 & -1.75961800 & 1.24655500 \\
\hline $\mathrm{H}$ & 5.91057400 & -4.29193200 & -1.02378300 & $\mathrm{H}$ & 3.36516400 & -0.91142700 & 0.85708800 \\
\hline $\mathrm{H}$ & 6.73630800 & -1.94635100 & -1.03687800 & & & & \\
\hline $\mathrm{H}$ & 5.22191300 & -0.09172500 & -0.43857200 & $6 \mathbf{a}$ & & & \\
\hline $\mathrm{H}$ & 1.98597500 & 1.48951700 & -1.99232400 & $\mathrm{Ru}$ & -0.42225900 & 0.50152800 & 0.24361500 \\
\hline $\mathrm{H}$ & 3.02748600 & 3.54206400 & -2.90291600 & $\mathrm{P}$ & -2.65247200 & -0.15882100 & -0.20140100 \\
\hline $\mathrm{H}$ & 4.72770200 & 4.77590000 & -1.56831000 & $\mathrm{P}$ & 1.78088800 & 1.30604900 & -0.00974700 \\
\hline $\mathrm{H}$ & 5.39460300 & 3.92031200 & 0.66995800 & $\mathrm{~N}$ & -0.23510700 & 0.20544000 & -1.78986300 \\
\hline
\end{tabular}




\begin{tabular}{|c|c|c|c|c|c|c|c|}
\hline $\mathrm{C}$ & -2.50785400 & -0.73251100 & -1.96664000 & $\mathrm{H}$ & -2.67855900 & 2.77169800 & 0.08763600 \\
\hline $\mathrm{C}$ & -1.42236800 & 0.10664100 & -2.64966400 & $\mathrm{H}$ & -4.46613400 & 4.48500700 & -0.08289500 \\
\hline $\mathrm{C}$ & 2.19157100 & 0.64285700 & -1.69999800 & $\mathrm{H}$ & -6.81660000 & 3.79269900 & -0.51034400 \\
\hline $\mathrm{C}$ & 0.91304200 & 0.72430100 & -2.54099700 & $\mathrm{H}$ & -7.36504200 & 1.37926600 & -0.76468000 \\
\hline $\mathrm{C}$ & -3.96747200 & -2.68682900 & 0.06734900 & $\mathrm{H}$ & -5.58660900 & -0.32209400 & -0.59241100 \\
\hline $\mathrm{C}$ & -4.55545300 & -3.71476400 & 0.80947700 & $\mathrm{H}$ & 4.47025100 & 0.00739000 & -0.43119100 \\
\hline $\mathrm{C}$ & -4.63546500 & -3.62022500 & 2.19895700 & $\mathrm{H}$ & 6.26427400 & -0.60648000 & 1.13460400 \\
\hline $\mathrm{C}$ & -4.12221200 & -2.49370000 & 2.84671300 & $\mathrm{H}$ & 6.01297300 & -0.16387000 & 3.56999800 \\
\hline $\mathrm{C}$ & -3.53046100 & -1.46960800 & 2.10866000 & $\mathrm{H}$ & 3.92630500 & 0.89200100 & 4.4227020 \\
\hline $\mathrm{C}$ & -3.44885300 & -1.55104700 & 0.70821500 & $\mathrm{H}$ & 2.11541400 & 1.49757100 & $2.8607970 \mathrm{C}$ \\
\hline $\mathrm{C}$ & -3.70284000 & 2.47163100 & -0.10956800 & $\mathrm{H}$ & 4.12451700 & 3.14007200 & 0.2872040 \\
\hline $\mathrm{C}$ & -4.71244000 & 3.43320400 & -0.20284400 & $\mathrm{H}$ & 4.41466700 & 5.55931900 & -0.10311400 \\
\hline $\mathrm{C}$ & -6.03071600 & 3.04511900 & -0.44154100 & $\mathrm{H}$ & 2.48470900 & 6.95133800 & -0.82756100 \\
\hline $\mathrm{C}$ & -6.33890800 & 1.68949400 & -0.58532900 & $\mathrm{H}$ & 0.25554700 & 5.89529800 & -1.15184800 \\
\hline $\mathrm{C}$ & -5.33209200 & 0.72960400 & -0.49278300 & $\mathrm{H}$ & -0.04048700 & 3.46837500 & -0.73535900 \\
\hline $\mathrm{C}$ & -3.99978300 & 1.11025300 & -0.25765200 & $\mathrm{C}$ & -0.40030900 & 0.19027300 & 2.09478600 \\
\hline $\mathrm{C}$ & 4.34145600 & 0.21391500 & 0.62627900 & $\mathrm{O}$ & -0.36845300 & -0.03885400 & 3.23783000 \\
\hline $\mathrm{C}$ & 5.36123700 & -0.13677600 & 1.51481700 & $\mathrm{H}$ & -0.88659200 & 1.93331400 & 0.70606200 \\
\hline $\mathrm{C}$ & 5.21902700 & 0.11019200 & 2.88033900 & $\mathrm{C}$ & 4.41989900 & -3.66098000 & 0.05582700 \\
\hline $\mathrm{C}$ & 4.04842000 & 0.70435500 & 3.35943000 & $\mathrm{C}$ & 3.03554200 & -3.54190800 & 0.18821600 \\
\hline $\mathrm{C}$ & 3.02710100 & 1.04981600 & 2.47595200 & $\mathrm{C}$ & 2.21668400 & -3.39486700 & -0.93944700 \\
\hline $\mathrm{C}$ & 3.16451000 & 0.81450600 & 1.09693300 & $\mathrm{C}$ & 2.81514700 & -3.35869100 & -2.20525600 \\
\hline $\mathrm{C}$ & 3.27431100 & 3.73585300 & -0.03094400 & $\mathrm{C}$ & 4.20079000 & -3.47407000 & -2.34071300 \\
\hline $\mathrm{C}$ & 3.43891700 & 5.10407800 & -0.25167000 & $\mathrm{C}$ & 5.00798000 & -3.62855900 & -1.21137400 \\
\hline $\mathrm{C}$ & 2.35537900 & 5.88565600 & -0.65786200 & $\mathrm{H}$ & 5.03900700 & -3.77151700 & 0.94250400 \\
\hline $\mathrm{C}$ & 1.10472800 & 5.29295900 & -0.83961600 & $\mathrm{H}$ & 2.58588000 & -3.55884400 & 1.17929300 \\
\hline $\mathrm{C}$ & 0.93636100 & 3.92596300 & -0.61173900 & $\mathrm{H}$ & 2.17904800 & -3.23352600 & -3.07591300 \\
\hline $\mathrm{C}$ & 2.02008700 & 3.13051800 & -0.20877700 & $\mathrm{H}$ & 4.65025700 & -3.44554400 & -3.33058400 \\
\hline $\mathrm{H}$ & -2.16859800 & -1.77287100 & -1.94177200 & $\mathrm{H}$ & 6.08603700 & -3.72099600 & -1.31703400 \\
\hline $\mathrm{H}$ & -3.46393800 & -0.67444000 & -2.49801900 & $\mathrm{C}$ & 0.69824700 & -3.31930400 & -0.79123900 \\
\hline $\mathrm{H}$ & -1.15923700 & -0.37537100 & -3.60632200 & $\mathrm{H}$ & 0.47208900 & -2.75774500 & 0.13164300 \\
\hline $\mathrm{H}$ & -1.81499300 & 1.10700800 & -2.90396800 & $\mathrm{O}$ & 0.08109300 & -2.68927400 & -1.90396700 \\
\hline $\mathrm{H}$ & 3.01940900 & 1.18657700 & -2.16845700 & $\mathrm{H}$ & 0.12087900 & -1.70865300 & -1.77218100 \\
\hline $\mathrm{H}$ & 2.48225400 & -0.40705100 & -1.58020300 & $\mathrm{C}$ & 0.08373800 & -4.71770900 & -0.66302100 \\
\hline $\mathrm{H}$ & 0.74477700 & 1.76878900 & -2.86025900 & $\mathrm{H}$ & 0.25864400 & -5.28674100 & -1.58288800 \\
\hline $\mathrm{H}$ & 1.05749800 & 0.14014000 & -3.46577700 & $\mathrm{H}$ & 0.52647300 & -5.26832500 & 0.17374700 \\
\hline $\mathrm{H}$ & -3.91596700 & -2.78008100 & -1.01268800 & $\mathrm{H}$ & -0.99644000 & -4.63632700 & -0.50198600 \\
\hline $\mathrm{H}$ & -4.94972300 & -4.58865300 & 0.29752200 & & & & \\
\hline $\mathrm{H}$ & -5.09070400 & -4.42123500 & 2.77517900 & $6 b$ & & & \\
\hline $\mathrm{H}$ & -4.17461200 & -2.41482000 & 3.92919000 & $\mathrm{Ru}$ & 0.22466200 & -0.47465800 & 0.04595200 \\
\hline $\mathrm{H}$ & -3.12056100 & -0.60721100 & 2.62603200 & $\mathrm{P}$ & -1.93808100 & -1.36146500 & 0.34273100 \\
\hline
\end{tabular}




\begin{tabular}{|c|c|c|c|c|c|c|c|}
\hline $\mathrm{P}$ & 2.53065500 & -0.05115100 & 0.36432100 & $\mathrm{H}$ & -6.85502400 & -1.10081100 & -0.53379400 \\
\hline $\mathrm{N}$ & 0.26288700 & -0.64547300 & 2.19586600 & $\mathrm{H}$ & -4.79919400 & -2.22685400 & 0.24769300 \\
\hline $\mathrm{C}$ & -2.11528000 & -1.29405000 & 2.19535700 & $\mathrm{H}$ & -1.52721500 & -2.70001300 & -2.17436800 \\
\hline $\mathrm{C}$ & -0.73352800 & -1.51248100 & 2.81957400 & $\mathrm{H}$ & -1.80384700 & -5.02061700 & -2.96927700 \\
\hline $\mathrm{C}$ & 2.61741100 & 0.08058700 & 2.22433600 & $\mathrm{H}$ & -2.53344400 & -6.80478300 & -1.39297100 \\
\hline $\mathrm{C}$ & 1.56808600 & -0.86426100 & 2.81667200 & $\mathrm{H}$ & -2.99967000 & -6.23271400 & 0.98194700 \\
\hline $\mathrm{C}$ & -3.42947000 & 0.68852700 & -0.86223900 & $\mathrm{H}$ & -2.75877300 & -3.91799100 & 1.77235600 \\
\hline $\mathrm{C}$ & -4.59306900 & 1.32244000 & -1.30632500 & $\mathrm{H}$ & 2.02593500 & 2.76983400 & 0.8818220 \\
\hline $\mathrm{C}$ & -5.82641800 & 0.68164400 & -1.18664700 & $\mathrm{H}$ & 3.04138000 & 4.87120600 & 0.0773210 \\
\hline $\mathrm{C}$ & -5.89654900 & -0.59572600 & -0.62228300 & $\mathrm{H}$ & 4.91058400 & 4.81068000 & -1.56657300 \\
\hline $\mathrm{C}$ & -4.73681900 & -1.22808700 & -0.17604100 & $\mathrm{H}$ & 5.75814900 & 2.61869200 & -2.38350400 \\
\hline $\mathrm{C}$ & -3.49104300 & -0.58808700 & -0.28665100 & $\mathrm{H}$ & 4.77884600 & 0.51755700 & -1.54794200 \\
\hline $\mathrm{C}$ & -1.86712600 & -3.46733800 & -1.48428500 & $\mathrm{H}$ & 5.31956300 & -0.63726200 & 1.21613700 \\
\hline $\mathrm{C}$ & -2.01551100 & -4.77880700 & -1.93110400 & $\mathrm{H}$ & 6.91509700 & -2.42214100 & 0.63617200 \\
\hline $\mathrm{C}$ & -2.42498800 & -5.78054300 & -1.04632600 & $\mathrm{H}$ & 6.28286900 & -4.18098900 & -1.00635100 \\
\hline $\mathrm{C}$ & -2.68739800 & -5.45957000 & 0.28470600 & $\mathrm{H}$ & 4.02729000 & -4.13602200 & -2.05471000 \\
\hline $\mathrm{C}$ & -2.54985300 & -4.14156900 & 0.73111000 & $\mathrm{H}$ & 2.40917400 & -2.36128000 & -1.44353900 \\
\hline $\mathrm{C}$ & -2.14049600 & -3.12908500 & -0.14755000 & $\mathrm{C}$ & 0.16176800 & -0.20019900 & -1.81032700 \\
\hline $\mathrm{C}$ & 2.86501300 & 2.73179300 & 0.19373600 & $\mathrm{O}$ & 0.13221900 & -0.08054200 & -2.96938700 \\
\hline $\mathrm{C}$ & 3.42808200 & 3.91855300 & -0.27528500 & $\mathrm{H}$ & 0.62339100 & -1.96654500 & -0.24199900 \\
\hline $\mathrm{C}$ & 4.47466900 & 3.88499000 & -1.20018800 & $\mathrm{C}$ & -3.12204000 & 5.23070300 & -0.47979500 \\
\hline $\mathrm{C}$ & 4.95032300 & 2.65590300 & -1.65721800 & $\mathrm{C}$ & -2.82416000 & 4.30720500 & 0.52174800 \\
\hline $\mathrm{C}$ & 4.39419300 & 1.46472300 & -1.18436400 & $\mathrm{C}$ & -1.62964800 & 3.57144900 & 0.49230500 \\
\hline $\mathrm{C}$ & 3.35180100 & 1.48791500 & -0.24594500 & $\mathrm{C}$ & -0.74363500 & 3.77429300 & -0.57158800 \\
\hline $\mathrm{C}$ & 5.02082100 & -1.40846600 & 0.51136300 & $\mathrm{C}$ & -1.04223000 & 4.69811200 & -1.57786900 \\
\hline $\mathrm{C}$ & 5.92995200 & -2.41171800 & 0.17721600 & $\mathrm{C}$ & -2.22843700 & 5.43080300 & -1.53601500 \\
\hline $\mathrm{C}$ & 5.57484800 & -3.39847000 & -0.74634600 & $\mathrm{H}$ & -4.05290700 & 5.79086800 & -0.43803700 \\
\hline $\mathrm{C}$ & 4.30980000 & -3.37363200 & -1.33368900 & $\mathrm{H}$ & -3.53292300 & 4.15056900 & 1.33247700 \\
\hline $\mathrm{C}$ & 3.39667700 & -2.37198700 & -0.99447300 & $\mathrm{H}$ & 0.17345300 & 3.19931800 & -0.61639800 \\
\hline $\mathrm{C}$ & 3.73901300 & -1.37943100 & -0.06541300 & $\mathrm{H}$ & -0.34303300 & 4.83892100 & -2.39818800 \\
\hline $\mathrm{H}$ & -2.46889800 & -0.27995300 & 2.41621100 & $\mathrm{H}$ & -2.45917400 & 6.14770600 & -2.31969100 \\
\hline $\mathrm{H}$ & -2.86595300 & -1.98877400 & 2.58728400 & $\mathrm{C}$ & -1.33235600 & 2.58616500 & 1.61284300 \\
\hline $\mathrm{H}$ & -0.79765100 & -1.31189900 & 3.90521800 & $\mathrm{H}$ & -2.21644700 & 1.93687400 & 1.72679800 \\
\hline $\mathrm{H}$ & -0.45822800 & -2.58048100 & 2.72467100 & $\mathrm{O}$ & -0.19748800 & 1.79053900 & 1.28567100 \\
\hline $\mathrm{H}$ & 3.62051900 & -0.13376500 & 2.60704200 & $\mathrm{H}$ & -0.10631800 & 1.00453200 & 1.93227600 \\
\hline $\mathrm{H}$ & 2.37294300 & 1.11657700 & 2.48114000 & $\mathrm{C}$ & -1.08620800 & 3.30454500 & 2.95008000 \\
\hline $\mathrm{H}$ & 1.90830400 & -1.91020800 & 2.69350800 & $\mathrm{H}$ & -0.20965600 & 3.95618100 & 2.86877300 \\
\hline $\mathrm{H}$ & 1.51164000 & -0.68952700 & 3.90703300 & $\mathrm{H}$ & -1.94641500 & 3.91773100 & 3.24041300 \\
\hline $\mathrm{H}$ & -2.47094400 & 1.18818300 & -0.96324600 & $\mathrm{H}$ & -0.90315400 & 2.57224500 & 3.74534700 \\
\hline
\end{tabular}




\begin{tabular}{|c|c|c|c|c|c|c|c|}
\hline \multicolumn{4}{|c|}{ imaginary frequency $=-309.55 \mathrm{~cm}^{-1}$} & $\mathrm{H}$ & -4.50653500 & -2.19787400 & -1.32988800 \\
\hline $\mathrm{Ru}$ & -0.33370600 & 0.34573100 & 0.09752900 & $\mathrm{H}$ & -5.72908300 & -4.01612700 & -0.20710700 \\
\hline $\mathrm{P}$ & -2.63879300 & -0.07429600 & -0.29013900 & $\mathrm{H}$ & -5.48964100 & -4.36183800 & 2.24380400 \\
\hline $\mathrm{P}$ & 1.90743200 & 1.05845000 & -0.20827300 & $\mathrm{H}$ & -4.00080500 & -2.86571600 & 3.56162000 \\
\hline $\mathrm{N}$ & -0.26300700 & 0.20041300 & -2.06980900 & $\mathrm{H}$ & -2.75885400 & -1.05100800 & 2.44171000 \\
\hline $\mathrm{C}$ & -2.63657900 & -0.41917100 & -2.11914300 & $\mathrm{H}$ & -2.48493500 & 2.55934600 & 1.04133600 \\
\hline $\mathrm{C}$ & -1.54642200 & 0.44236000 & -2.76212600 & $\mathrm{H}$ & -4.10966800 & 4.40509200 & 1.35141400 \\
\hline $\mathrm{C}$ & 2.16369500 & 0.62328900 & -1.99923900 & $\mathrm{H}$ & -6.39811200 & 4.22444700 & 0.39355300 \\
\hline $\mathrm{C}$ & 0.85188100 & 0.90171100 & -2.73788000 & $\mathrm{H}$ & -7.05460000 & 2.17343500 & -0.85318500 \\
\hline $\mathrm{C}$ & -4.39581400 & -2.32707800 & -0.25736300 & $\mathrm{H}$ & -5.45143600 & 0.32551400 & -1.13050600 \\
\hline $\mathrm{C}$ & -5.08805100 & -3.36088700 & 0.37664200 & $\mathrm{H}$ & 4.56168100 & -0.12204600 & -1.01966300 \\
\hline $\mathrm{C}$ & -4.95256400 & -3.55538000 & 1.75195800 & $\mathrm{H}$ & 6.47406000 & -0.92915100 & 0.30012600 \\
\hline $\mathrm{C}$ & -4.11761200 & -2.71523700 & 2.49184600 & $\mathrm{H}$ & 6.37458600 & -0.92953100 & 2.78491400 \\
\hline $\mathrm{C}$ & -3.41965000 & -1.68654700 & 1.85935700 & $\mathrm{H}$ & 4.31773600 & -0.13190100 & 3.93744800 \\
\hline $\mathrm{C}$ & -3.55533700 & -1.47688900 & 0.47788100 & $\mathrm{H}$ & 2.39139200 & 0.67052300 & 2.62224300 \\
\hline $\mathrm{C}$ & -3.48416500 & 2.47614500 & 0.62769100 & $\mathrm{H}$ & 4.28637300 & 2.64697900 & -0.93770900 \\
\hline $\mathrm{C}$ & -4.40192000 & 3.51606800 & 0.79881400 & $\mathrm{H}$ & 4.80609200 & 5.05381000 & -0.88685700 \\
\hline $\mathrm{C}$ & -5.68538000 & 3.41449800 & 0.26303200 & $\mathrm{H}$ & 3.13041000 & 6.67859600 & -0.02309300 \\
\hline $\mathrm{C}$ & -6.05405200 & 2.26325200 & -0.43848000 & $\mathrm{H}$ & 0.92241800 & 5.86156400 & 0.77952700 \\
\hline $\mathrm{C}$ & -5.14079900 & 1.22320500 & -0.60274200 & $\mathrm{H}$ & 0.38829100 & 3.44289000 & 0.69611100 \\
\hline $\mathrm{C}$ & -3.84008800 & 1.32029200 & -0.07947900 & $\mathrm{C}$ & -0.38167800 & 0.39786900 & 1.96553800 \\
\hline $\mathrm{C}$ & 4.50157000 & -0.10398500 & 0.06390700 & $\mathrm{O}$ & -0.41948900 & 0.45460200 & 3.12935300 \\
\hline $\mathrm{C}$ & 5.58667600 & -0.56609100 & 0.81121900 & $\mathrm{H}$ & -0.72998400 & 1.87270300 & 0.04914600 \\
\hline $\mathrm{C}$ & 5.52848500 & -0.57055500 & 2.20500100 & $\mathrm{C}$ & 3.61862300 & -3.73273900 & 0.78227600 \\
\hline $\mathrm{C}$ & 4.37468000 & -0.12199300 & 2.85228100 & $\mathrm{C}$ & 2.32183800 & -3.24547300 & 0.61893700 \\
\hline $\mathrm{C}$ & 3.28769400 & 0.33492300 & 2.10830300 & $\mathrm{C}$ & 1.78171500 & -3.05722000 & -0.66090200 \\
\hline $\mathrm{C}$ & 3.34331100 & 0.35770000 & 0.70507900 & $\mathrm{C}$ & 2.57543500 & -3.35538300 & -1.77442700 \\
\hline $\mathrm{C}$ & 3.53477400 & 3.34580900 & -0.58034100 & $\mathrm{C}$ & 3.87614400 & -3.84165600 & -1.61487500 \\
\hline $\mathrm{C}$ & 3.83574000 & 4.70604400 & -0.54238900 & $\mathrm{C}$ & 4.40091600 & -4.03626900 & -0.33614600 \\
\hline $\mathrm{C}$ & 2.89503400 & 5.61810900 & -0.05567500 & $\mathrm{H}$ & 4.02249100 & -3.86933000 & 1.78239000 \\
\hline $\mathrm{C}$ & 1.65703700 & 5.15989700 & 0.39324900 & $\mathrm{H}$ & 1.72384200 & -3.00610300 & 1.49603400 \\
\hline $\mathrm{C}$ & 1.35326600 & 3.79629000 & 0.34915000 & $\mathrm{H}$ & 2.13827100 & -3.20964900 & -2.75718300 \\
\hline $\mathrm{C}$ & 2.28469100 & 2.87361300 & -0.14362100 & $\mathrm{H}$ & 4.47673700 & -4.07709300 & -2.49103600 \\
\hline $\mathrm{H}$ & -2.35213600 & -1.46972900 & -2.24659100 & $\mathrm{H}$ & 5.41052600 & -4.41974200 & -0.20916700 \\
\hline $\mathrm{H}$ & -3.61107900 & -0.24205200 & -2.58553100 & $\mathrm{C}$ & 0.32463200 & -2.61336300 & -0.88226700 \\
\hline $\mathrm{H}$ & -1.45367200 & 0.17160300 & -3.82563800 & $\mathrm{H}$ & 0.17912800 & -1.68043900 & -0.15971100 \\
\hline $\mathrm{H}$ & -1.80360600 & 1.50890600 & -2.71365400 & $\mathrm{O}$ & 0.04031800 & -2.28598400 & -2.15909200 \\
\hline $\mathrm{H}$ & 2.99361800 & 1.17724200 & -2.45066900 & $\mathrm{H}$ & -0.06978900 & -0.95206900 & -2.17845200 \\
\hline $\mathrm{H}$ & 2.38663000 & -0.44856700 & -2.04786700 & $\mathrm{C}$ & -0.63459400 & -3.68362100 & -0.31482100 \\
\hline $\mathrm{H}$ & 0.64900000 & 1.98109400 & -2.76698700 & $\mathrm{H}$ & -0.49678100 & -4.61048900 & -0.88362200 \\
\hline $\mathrm{H}$ & 0.93846900 & 0.55514500 & -3.77959400 & $\mathrm{H}$ & -0.45056700 & -3.89469400 & 0.74479100 \\
\hline
\end{tabular}




\begin{tabular}{|c|c|c|c|c|c|c|c|}
\hline \multirow[t]{2}{*}{$\mathrm{H}$} & \multirow[t]{2}{*}{-1.67137400} & \multirow[t]{2}{*}{-3.35927600} & \multirow[t]{2}{*}{-0.43241400} & $\mathrm{H}$ & 2.43808900 & 0.97802300 & 2.46768800 \\
\hline & & & & $\mathrm{H}$ & 1.76244900 & -2.00964400 & 2.67694800 \\
\hline \multicolumn{4}{|c|}{ TS3b } & $\mathrm{H}$ & 1.48127700 & -0.77077100 & 3.90746200 \\
\hline \multicolumn{4}{|c|}{ imaginary frequency $=-990.05 \mathrm{~cm}^{-1}$} & $\mathrm{H}$ & -2.43028100 & 1.37569300 & -0.97348400 \\
\hline $\mathrm{Ru}$ & 0.17836200 & -0.44857200 & 0.03252800 & $\mathrm{H}$ & -4.42056500 & 2.60825900 & -1.76025200 \\
\hline $\mathrm{P}$ & -2.03818200 & -1.19576400 & 0.33686900 & $\mathrm{H}$ & -6.68498100 & 1.58875100 & -1.54844100 \\
\hline $\mathrm{P}$ & 2.51215400 & -0.18394100 & 0.34567400 & $\mathrm{H}$ & -6.93333600 & -0.66781200 & -0.53411800 \\
\hline $\mathrm{N}$ & 0.21486900 & -0.63548500 & 2.21130600 & $\mathrm{H}$ & -4.94272600 & -1.90187800 & 0.25202500 \\
\hline $\mathrm{C}$ & -2.21015200 & -1.10433700 & 2.19140800 & $\mathrm{H}$ & -1.70813500 & -2.58674500 & -2.16321400 \\
\hline $\mathrm{C}$ & -0.85544500 & -1.41631900 & 2.83515400 & $\mathrm{H}$ & -2.12958100 & -4.89393600 & -2.93285100 \\
\hline $\mathrm{C}$ & 2.61331600 & -0.07048500 & 2.20839900 & $\mathrm{H}$ & -2.96896800 & -6.61173400 & -1.33757900 \\
\hline $\mathrm{C}$ & 1.51334200 & -0.94448100 & 2.81725500 & $\mathrm{H}$ & -3.39953700 & -5.98525300 & 1.03037500 \\
\hline $\mathrm{C}$ & -3.41392300 & 0.92819600 & -0.87315400 & $\mathrm{H}$ & -3.01663000 & -3.68171600 & 1.79490600 \\
\hline $\mathrm{C}$ & -4.54120400 & 1.62263100 & -1.32023000 & $\mathrm{H}$ & 2.17346800 & 2.64689900 & 0.89148400 \\
\hline $\mathrm{C}$ & -5.80785600 & 1.05147500 & -1.19704600 & $\mathrm{H}$ & 3.32466900 & 4.69644200 & 0.12385700 \\
\hline $\mathrm{C}$ & -5.94844900 & -0.21704200 & -0.62573800 & $\mathrm{H}$ & 5.20133900 & 4.54195600 & -1.50514500 \\
\hline $\mathrm{C}$ & -4.82530900 & -0.91020200 & -0.17658700 & $\mathrm{H}$ & 5.91903700 & 2.31349400 & -2.34666800 \\
\hline $\mathrm{C}$ & -3.54634700 & -0.33982300 & -0.29122700 & $\mathrm{H}$ & 4.80521700 & 0.26530000 & -1.54776200 \\
\hline $\mathrm{C}$ & -2.09569700 & -3.32357500 & -1.46511100 & $\mathrm{H}$ & 5.26498900 & -0.94653600 & 1.17588500 \\
\hline $\mathrm{C}$ & -2.32570900 & -4.62797600 & -1.89755600 & $\mathrm{H}$ & 6.73970600 & -2.82784900 & 0.58156600 \\
\hline $\mathrm{C}$ & -2.79688400 & -5.59251100 & -1.00214500 & $\mathrm{H}$ & 5.98593900 & -4.53899400 & -1.06002200 \\
\hline $\mathrm{C}$ & -3.03912400 & -5.24105300 & 0.32492400 & $\mathrm{H}$ & 3.73042100 & -4.34892500 & -2.09206600 \\
\hline $\mathrm{C}$ & -2.81992200 & -3.92912500 & 0.75657400 & $\mathrm{H}$ & 2.23130100 & -2.47728100 & -1.46399800 \\
\hline $\mathrm{C}$ & -2.34765600 & -2.95393500 & -0.13256400 & $\mathrm{C}$ & 0.12491500 & -0.15075600 & -1.81256200 \\
\hline $\mathrm{C}$ & 3.01704200 & 2.57088100 & 0.21182100 & $\mathrm{O}$ & 0.09685500 & -0.00827500 & -2.96838800 \\
\hline $\mathrm{C}$ & 3.65606500 & 3.72638900 & -0.23726900 & $\mathrm{H}$ & 0.47053100 & -1.96744000 & -0.29135400 \\
\hline $\mathrm{C}$ & 4.70634300 & 3.64015300 & -1.15453600 & $\mathrm{C}$ & -2.70800800 & 5.28940000 & -0.46154100 \\
\hline $\mathrm{C}$ & 5.10939000 & 2.39040000 & -1.62554700 & $\mathrm{C}$ & -2.45291800 & 4.36837900 & 0.55414600 \\
\hline $\mathrm{C}$ & 4.47720100 & 1.22952000 & -1.17358300 & $\mathrm{C}$ & -1.32877600 & 3.52920400 & 0.50472900 \\
\hline $\mathrm{C}$ & 3.43117200 & 1.30597700 & -0.24204800 & $\mathrm{C}$ & -0.47140700 & 3.62865200 & -0.59632000 \\
\hline $\mathrm{C}$ & 4.91256100 & -1.69604200 & 0.47242600 & $\mathrm{C}$ & -0.72585100 & 4.54938000 & -1.61783400 \\
\hline $\mathrm{C}$ & 5.75391800 & -2.75407400 & 0.12989200 & $\mathrm{C}$ & -1.84147400 & 5.38446400 & -1.55476300 \\
\hline $\mathrm{C}$ & 5.33061300 & -3.71401100 & -0.79325200 & $\mathrm{H}$ & -3.58449300 & 5.93030700 & -0.40208900 \\
\hline $\mathrm{C}$ & 4.06560200 & -3.60775700 & -1.37148900 & $\mathrm{H}$ & -3.14185400 & 4.29458300 & 1.39346700 \\
\hline $\mathrm{C}$ & 3.22001000 & -2.55126000 & -1.02370000 & $\mathrm{H}$ & 0.38832200 & 2.97225800 & -0.65452600 \\
\hline $\mathrm{C}$ & 3.63111000 & -1.58453500 & -0.09536700 & $\mathrm{H}$ & -0.04836600 & 4.60797100 & -2.46613200 \\
\hline $\mathrm{H}$ & -2.49809600 & -0.06854400 & 2.40401400 & $\mathrm{H}$ & -2.03873800 & 6.09942800 & -2.34945400 \\
\hline $\mathrm{H}$ & -3.00745200 & -1.74380200 & 2.58496300 & $\mathrm{C}$ & -1.07130600 & 2.54265400 & 1.64072000 \\
\hline $\mathrm{H}$ & -0.91170300 & -1.20176500 & 3.91717600 & $\mathrm{H}$ & -2.01928500 & 1.99571400 & 1.80524600 \\
\hline $\mathrm{H}$ & -0.64472400 & -2.49507900 & 2.74240500 & $\mathrm{O}$ & -0.03105700 & 1.64086200 & 1.33952500 \\
\hline $\mathrm{H}$ & 3.60226900 & -0.35160100 & 2.58400900 & $\mathrm{H}$ & 0.01135900 & 0.68443400 & 2.02913300 \\
\hline
\end{tabular}




\begin{tabular}{|c|c|c|c|c|c|c|c|}
\hline $\mathrm{C}$ & -0.73338500 & 3.28684400 & 2.94772100 & $\mathrm{H}$ & -0.86319500 & -2.67352100 & 2.57936500 \\
\hline $\mathrm{H}$ & 0.19560300 & 3.85426700 & 2.82353100 & $\mathrm{H}$ & 3.60684200 & -0.93411400 & 2.54566500 \\
\hline $\mathrm{H}$ & -1.52695500 & 3.98467300 & 3.23827700 & $\mathrm{H}$ & 2.54468000 & 0.47915000 & 2.62900800 \\
\hline \multirow[t]{2}{*}{$\mathrm{H}$} & -0.59235000 & 2.56747700 & 3.76313300 & $\mathrm{H}$ & 1.60621000 & -2.44378600 & 2.44847400 \\
\hline & & & & $\mathrm{H}$ & 1.52088100 & -1.40414200 & 3.88280900 \\
\hline 7 & & & & $\mathrm{H}$ & -2.41700500 & 1.51474700 & -1.07933900 \\
\hline $\mathrm{Ru}$ & 0.13074600 & -0.40442400 & 0.11262700 & $\mathrm{H}$ & -4.35282000 & 2.87179100 & -1.78429500 \\
\hline $\mathrm{P}$ & -2.13881300 & -0.99047000 & 0.39781000 & $\mathrm{H}$ & -6.66726700 & 2.13358300 & -1.22686700 \\
\hline $\mathrm{P}$ & 2.49016500 & -0.39253400 & 0.37344000 & $\mathrm{H}$ & -7.01803800 & 0.02463600 & 0.04390000 \\
\hline $\mathrm{N}$ & 0.20220000 & -0.89715800 & 2.29031200 & $\mathrm{H}$ & -5.08432500 & -1.34608900 & 0.72969800 \\
\hline $\mathrm{C}$ & -2.25992800 & -1.04090300 & 2.26111800 & $\mathrm{H}$ & -1.78154900 & -2.31394500 & -2.13617500 \\
\hline $\mathrm{C}$ & -0.96341000 & -1.61369400 & 2.84105600 & $\mathrm{H}$ & -2.42555900 & -4.51527700 & -3.05113800 \\
\hline $\mathrm{C}$ & 2.63433000 & -0.53900700 & 2.23620400 & $\mathrm{H}$ & -3.56679200 & -6.18742400 & -1.60156800 \\
\hline $\mathrm{C}$ & 1.49755200 & -1.40501100 & 2.78123400 & $\mathrm{H}$ & -4.07913200 & -5.61751800 & 0.76464500 \\
\hline $\mathrm{C}$ & -3.42080200 & 1.18266100 & -0.83705900 & $\mathrm{H}$ & -3.48464800 & -3.41306500 & 1.66715400 \\
\hline $\mathrm{C}$ & -4.51936500 & 1.94996700 & -1.23500500 & $\mathrm{H}$ & 2.25969500 & 2.35979300 & 1.14924100 \\
\hline $\mathrm{C}$ & -5.81281500 & 1.53747800 & -0.91702200 & $\mathrm{H}$ & 3.62028000 & 4.39930000 & 0.73283500 \\
\hline $\mathrm{C}$ & -6.01104000 & 0.35239000 & -0.20116000 & $\mathrm{H}$ & 5.62968600 & 4.28019900 & -0.73124800 \\
\hline $\mathrm{C}$ & -4.91725600 & -0.41546700 & 0.19379600 & $\mathrm{H}$ & 6.27623600 & 2.11098700 & -1.76199200 \\
\hline $\mathrm{C}$ & -3.60970800 & -0.00278500 & -0.11525100 & $\mathrm{H}$ & 4.96300100 & 0.07596400 & -1.30227500 \\
\hline $\mathrm{C}$ & -2.30323700 & -3.02706800 & -1.50462900 & $\mathrm{H}$ & 5.15272500 & -1.55444900 & 1.05733800 \\
\hline $\mathrm{C}$ & -2.65785900 & -4.27341200 & -2.01743700 & $\mathrm{H}$ & 6.41531400 & -3.47927800 & 0.18380300 \\
\hline $\mathrm{C}$ & -3.29853000 & -5.21225200 & -1.20409500 & $\mathrm{H}$ & 5.48612000 & -4.84636800 & -1.67589600 \\
\hline $\mathrm{C}$ & -3.58648100 & -4.89245200 & 0.12195000 & $\mathrm{H}$ & 3.26690200 & -4.26982000 & -2.64108100 \\
\hline $\mathrm{C}$ & -3.24400900 & -3.63656800 & 0.63220600 & $\mathrm{H}$ & 1.97307900 & -2.36239500 & -1.72731800 \\
\hline $\mathrm{C}$ & -2.59894800 & -2.68772700 & -0.17360600 & $\mathrm{C}$ & 0.07738400 & 0.02378800 & -1.68942800 \\
\hline $\mathrm{C}$ & 3.17069300 & 2.30492500 & 0.55724800 & $\mathrm{O}$ & 0.04790800 & 0.23876200 & -2.83448100 \\
\hline $\mathrm{C}$ & 3.92322100 & 3.45039200 & 0.29810000 & $\mathrm{H}$ & 0.28375700 & -1.94460400 & -0.32938300 \\
\hline $\mathrm{C}$ & 5.04726600 & 3.38500600 & -0.52914700 & $\mathrm{C}$ & -2.14712700 & 5.28670900 & -0.89259400 \\
\hline $\mathrm{C}$ & 5.41108900 & 2.16791700 & -1.10638700 & $\mathrm{C}$ & -2.03666900 & 4.38422000 & 0.16538200 \\
\hline $\mathrm{C}$ & 4.66667900 & 1.01474900 & -0.84627500 & $\mathrm{C}$ & -0.95985500 & 3.48619500 & 0.24562100 \\
\hline $\mathrm{C}$ & 3.54795700 & 1.07048600 & -0.00204600 & $\mathrm{C}$ & -0.00200500 & 3.51126200 & -0.77286300 \\
\hline $\mathrm{C}$ & 4.72116500 & -2.15391200 & 0.26027000 & $\mathrm{C}$ & -0.10943500 & 4.41147300 & -1.83793000 \\
\hline $\mathrm{C}$ & 5.44447500 & -3.23647100 & -0.24016500 & $\mathrm{C}$ & -1.17954300 & 5.30414400 & -1.90229800 \\
\hline $\mathrm{C}$ & 4.92283400 & -4.00282200 & -1.28558900 & $\mathrm{H}$ & -2.98832000 & 5.97550800 & -0.93120700 \\
\hline $\mathrm{C}$ & 3.67801700 & -3.67978300 & -1.82639300 & $\mathrm{H}$ & -2.80312900 & 4.37077000 & 0.93844400 \\
\hline $\mathrm{C}$ & 2.94965800 & -2.60071300 & -1.31982400 & $\mathrm{H}$ & 0.82375400 & 2.81208800 & -0.72446500 \\
\hline $\mathrm{C}$ & 3.46063800 & -1.82548200 & -0.26906000 & $\mathrm{H}$ & 0.64611000 & 4.41012100 & -2.62014300 \\
\hline $\mathrm{H}$ & -2.38103900 & 0.00334800 & 2.57203700 & $\mathrm{H}$ & -1.26386300 & 6.00369200 & -2.73032500 \\
\hline $\mathrm{H}$ & -3.13249800 & -1.58942300 & 2.63054900 & $\mathrm{C}$ & -0.84646000 & 2.51486400 & 1.43014600 \\
\hline $\mathrm{H}$ & -0.97665700 & -1.54407500 & 3.94038500 & $\mathrm{H}$ & -1.85818500 & 2.06470200 & 1.54390800 \\
\hline
\end{tabular}




\begin{tabular}{|c|c|c|c|c|c|c|c|}
\hline $\mathrm{O}$ & 0.13483100 & 1.54298300 & 1.29506400 & $\mathrm{H}$ & -3.61109100 & -0.23569800 & -2.58428000 \\
\hline $\mathrm{H}$ & 0.13020600 & 0.12793600 & 2.46301700 & $\mathrm{H}$ & -1.46720600 & 0.20290100 & -3.83081600 \\
\hline $\mathrm{C}$ & -0.57845000 & 3.31652600 & 2.72642600 & $\mathrm{H}$ & -1.79996800 & 1.52591100 & -2.69486900 \\
\hline $\mathrm{H}$ & 0.39574800 & 3.81499200 & 2.66063800 & $\mathrm{H}$ & 2.99909700 & 1.17222400 & -2.45278700 \\
\hline $\mathrm{H}$ & -1.34262000 & 4.08063400 & 2.91289600 & $\mathrm{H}$ & 2.38473200 & -0.45327600 & -2.06149300 \\
\hline \multirow[t]{2}{*}{$\mathrm{H}$} & -0.55516300 & 2.63345800 & 3.58475900 & $\mathrm{H}$ & 0.65312300 & 1.99059600 & -2.75152500 \\
\hline & & & & $\mathrm{H}$ & 0.94267300 & 0.58139800 & -3.78851800 \\
\hline 8 & & & & $\mathrm{H}$ & -4.50146100 & -2.18989900 & -1.33906600 \\
\hline $\mathrm{Ru}$ & -0.32717100 & 0.33466500 & 0.09623500 & $\mathrm{H}$ & -5.74646000 & -3.99430700 & -0.21867400 \\
\hline $\mathrm{P}$ & -2.63617200 & -0.06791400 & -0.28949100 & $\mathrm{H}$ & -5.53693900 & -4.32533500 & 2.23698200 \\
\hline$P$ & 1.91545900 & 1.04204000 & -0.20877200 & $\mathrm{H}$ & -4.05547900 & -2.82843400 & 3.56221300 \\
\hline $\mathrm{N}$ & -0.26148900 & 0.20545400 & -2.08362000 & $\mathrm{H}$ & -2.79149400 & -1.02736700 & 2.44520800 \\
\hline $\mathrm{C}$ & -2.63520600 & -0.40887200 & -2.11935900 & $\mathrm{H}$ & -2.46008600 & 2.57498100 & 1.02449700 \\
\hline $\mathrm{C}$ & -1.55132700 & 0.45947200 & -2.76355400 & $\mathrm{H}$ & -4.07341700 & 4.43183900 & 1.33045400 \\
\hline $\mathrm{C}$ & 2.16683100 & 0.61937300 & -2.00438200 & $\mathrm{H}$ & -6.36750500 & 4.25733200 & 0.38504500 \\
\hline $\mathrm{C}$ & 0.85767700 & 0.91223800 & -2.74205300 & $\mathrm{H}$ & -7.04148800 & 2.20123600 & -0.84396200 \\
\hline $\mathrm{C}$ & -4.40425100 & -2.31224700 & -0.26447100 & $\mathrm{H}$ & -5.45010300 & 0.34216200 & -1.11535100 \\
\hline $\mathrm{C}$ & -5.10897800 & -3.33845200 & 0.36825000 & $\mathrm{H}$ & 4.57365900 & -0.12003300 & -1.03710600 \\
\hline $\mathrm{C}$ & -4.99022300 & -3.52474500 & 1.74618000 & $\mathrm{H}$ & 6.49307000 & -0.92750100 & 0.27208000 \\
\hline $\mathrm{C}$ & -4.15942200 & -2.68409200 & 2.49027400 & $\mathrm{H}$ & 6.39751200 & -0.95205300 & 2.75685500 \\
\hline $\mathrm{C}$ & -3.44914600 & -1.66305500 & 1.85933500 & $\mathrm{H}$ & 4.33719500 & -0.17920500 & 3.92002400 \\
\hline $\mathrm{C}$ & -3.56803500 & -1.46176800 & 0.47504100 & $\mathrm{H}$ & 2.40377700 & 0.62368200 & 2.61536300 \\
\hline $\mathrm{C}$ & -3.46208000 & 2.49448100 & 0.61709600 & $\mathrm{H}$ & 4.29347700 & 2.63124100 & -0.94449800 \\
\hline $\mathrm{C}$ & -4.37328800 & 3.54064900 & 0.78545600 & $\mathrm{H}$ & 4.81920900 & 5.03607200 & -0.87954000 \\
\hline C & -5.65989900 & 3.44255000 & 0.25667900 & $\mathrm{H}$ & 3.15420800 & 6.65826500 & 0.00948300 \\
\hline $\mathrm{C}$ & -6.03839000 & 2.28844900 & -0.43489200 & $\mathrm{H}$ & 0.95026000 & 5.84034400 & 0.82235600 \\
\hline $\mathrm{C}$ & -5.13179100 & 1.24214000 & -0.59608000 & $\mathrm{H}$ & 0.40887600 & 3.42360800 & 0.72247000 \\
\hline $\mathrm{C}$ & -3.82785100 & 1.33571300 & -0.08004800 & $\mathrm{C}$ & -0.37404300 & 0.38208300 & 1.96182200 \\
\hline $\mathrm{C}$ & 4.51500900 & -0.11267700 & 0.04669600 & $\mathrm{O}$ & -0.41065000 & 0.43326500 & 3.12582700 \\
\hline $\mathrm{C}$ & 5.60415000 & -0.57501900 & 0.78791400 & $\mathrm{H}$ & -0.71616600 & 1.86881200 & 0.04410200 \\
\hline $\mathrm{C}$ & 5.54817700 & -0.59311200 & 2.18167600 & $\mathrm{C}$ & 3.60632200 & -3.71129600 & 0.77628400 \\
\hline $\mathrm{C}$ & 4.39245700 & -0.15838200 & 2.83493100 & $\mathrm{C}$ & 2.30711400 & -3.22823700 & 0.61975800 \\
\hline $\mathrm{C}$ & 3.30146400 & 0.29875500 & 2.09698100 & $\mathrm{C}$ & 1.75636900 & -3.05104700 & -0.65711600 \\
\hline $\mathrm{C}$ & 3.35497600 & 0.33556200 & 0.69402900 & $\mathrm{C}$ & 2.54255300 & -3.35482100 & -1.77447800 \\
\hline $\mathrm{C}$ & 3.54582700 & 3.32893400 & -0.57682300 & $\mathrm{C}$ & 3.84607800 & -3.83580400 & -1.62199500 \\
\hline $\mathrm{C}$ & 3.85051600 & 4.68809700 & -0.53063700 & $\mathrm{C}$ & 4.38098900 & -4.02041600 & -0.34600100 \\
\hline $\mathrm{C}$ & 2.91584300 & 5.59867100 & -0.02973400 & $\mathrm{H}$ & 4.01822400 & -3.84025000 & 1.77414700 \\
\hline $\mathrm{C}$ & 1.68011500 & 5.13995600 & 0.42487500 & $\mathrm{H}$ & 1.71578900 & -2.98391700 & 1.49985100 \\
\hline $\mathrm{C}$ & 1.37238200 & 3.77753300 & 0.37203200 & $\mathrm{H}$ & 2.09481800 & -3.21955700 & -2.75405800 \\
\hline $\mathrm{C}$ & 2.29768700 & 2.85637900 & -0.13487400 & $\mathrm{H}$ & 4.44045800 & -4.07614200 & -2.50111700 \\
\hline $\mathrm{H}$ & -2.34241900 & -1.45739400 & -2.24864100 & $\mathrm{H}$ & 5.39243900 & -4.40071700 & -0.22399500 \\
\hline
\end{tabular}




$\mathrm{C}$
$\mathrm{H}$
$\mathrm{O}$
$\mathrm{H}$
$\mathrm{C}$
$\mathrm{H}$
$\mathrm{H}$
$\mathrm{H}$

$\begin{array}{rrr}0.29401100 & -2.61805500 & -0.87790800 \\ 0.16071700 & -1.65117100 & -0.16479300 \\ -0.00704100 & -2.32753400 & -2.14818000 \\ -0.08263300 & -0.89693100 & -2.19576000 \\ -0.65745000 & -3.66242000 & -0.24962600 \\ -0.52330200 & -4.61386800 & -0.77759000 \\ -0.46368500 & -3.82505400 & 0.81669400 \\ -1.69525200 & -3.34479300 & -0.37327400\end{array}$

C

$\mathrm{H}$

$\mathrm{H}$

$\mathrm{H}$

$\mathrm{H}$

$\mathrm{H}$

H

$\mathrm{H}$

$\mathrm{H}$

TS4

imaginary frequency $=-453.13 \mathrm{~cm}^{-1}$

\begin{tabular}{|c|c|c|c|}
\hline $\mathrm{Ru}$ & -0.31224900 & 0.33252000 & 0.08371600 \\
\hline $\mathrm{P}$ & -2.61794300 & -0.02467900 & -0.29337800 \\
\hline $\mathrm{P}$ & 1.92726100 & 1.00533600 & -0.20513000 \\
\hline $\mathrm{N}$ & -0.23590500 & 0.15999100 & -2.12943700 \\
\hline $\mathrm{C}$ & -2.63512000 & -0.38359300 & -2.12395700 \\
\hline $\mathrm{C}$ & -1.53317900 & 0.44465700 & -2.78922000 \\
\hline $\mathrm{C}$ & 2.19463500 & 0.60363500 & -2.00872100 \\
\hline $\mathrm{C}$ & 0.89116100 & 0.88372400 & -2.76137700 \\
\hline $\mathrm{C}$ & -4.40477100 & -2.26445200 & -0.26266300 \\
\hline $\mathrm{C}$ & -5.13052500 & -3.27143700 & 0.37854700 \\
\hline $\mathrm{C}$ & -5.05094400 & -3.41969400 & 1.76358100 \\
\hline $\mathrm{C}$ & -4.23821600 & -2.55975700 & 2.50628000 \\
\hline $\mathrm{C}$ & -3.50735200 & -1.55888100 & 1.86708100 \\
\hline $\mathrm{C}$ & -3.58572800 & -1.39547200 & 0.47442500 \\
\hline $\mathrm{C}$ & -3.41373300 & 2.59439800 & 0.47923100 \\
\hline $\mathrm{C}$ & -4.32134200 & 3.64781800 & 0.62181400 \\
\hline $\mathrm{C}$ & -5.63549300 & 3.50806800 & 0.17691500 \\
\hline $\mathrm{C}$ & -6.04486600 & 2.30498200 & -0.40511500 \\
\hline $\mathrm{C}$ & -5.14204900 & 1.25152800 & -0.53893200 \\
\hline $\mathrm{C}$ & -3.81121500 & 1.38607100 & -0.10657500 \\
\hline $\mathrm{C}$ & 4.55906100 & -0.09223100 & 0.04469500 \\
\hline $\mathrm{C}$ & 5.64546800 & -0.56365700 & 0.78428200 \\
\hline $\mathrm{C}$ & 5.55270400 & -0.67959700 & 2.17161400 \\
\hline $\mathrm{C}$ & 4.36292000 & -0.33455100 & 2.81685300 \\
\hline $\mathrm{C}$ & 3.27497900 & 0.13149800 & 2.07890100 \\
\hline $\mathrm{C}$ & 3.36431500 & 0.26858000 & 0.68426300 \\
\hline $\mathrm{C}$ & 3.61340600 & 3.27054800 & -0.54116300 \\
\hline $\mathrm{C}$ & 3.94427000 & 4.62258500 & -0.46707400 \\
\hline $\mathrm{C}$ & 3.02948400 & 5.53938300 & 0.05838200 \\
\hline $\mathrm{C}$ & 1.78681700 & 5.09368400 & 0.50722300 \\
\hline $\mathrm{C}$ & 1.45197200 & 3.73920400 & 0.42353900 \\
\hline
\end{tabular}

\begin{tabular}{|c|c|c|}
\hline 3700 & 2.81172000 & -0.10 \\
\hline .38896000 & -1.44468600 & -2.24374700 \\
\hline .60754900 & -0.17795600 & -2.58340800 \\
\hline .47502300 & 0.19436500 & -3.8596130 \\
\hline 1.73579800 & 1.51760300 & -2.7021860 \\
\hline 3.01742900 & 1.17725700 & -2.4487210 \\
\hline 2.44280000 & -0.46221100 & -2.0728690 \\
\hline 0.66153100 & 1.95483200 & -2.7417370 \\
\hline 0.99210900 & 0.58134600 & -3.8150070 \\
\hline-4.47436200 & -2.17143300 & -1.34234000 \\
\hline .75470700 & -3.94082100 & -0.2076150 \\
\hline 5.61426400 & -4.20435100 & 2.2614320 \\
\hline 4.16510200 & -2.67345500 & 3.5845220 \\
\hline 2.86283600 & -0.90982900 & 2.4531140 \\
\hline 2.38741500 & 2.70495300 & 0.8125980 \\
\hline 3.99662000 & 4.57794700 & 1.0810050 \\
\hline .34027300 & 4.32832500 & 0.2855240 \\
\hline-7.06912100 & 2.18511000 & -0.7485640 \\
\hline 5.48358500 & 0.31392400 & -0.9694450 \\
\hline 4.64993400 & -0.01857500 & -1.0348340 \\
\hline 6.56278500 & -0.84321900 & 0.27 \\
\hline 6.40045200 & -1.04272100 & 2.7466970 \\
\hline 4.27873100 & -0.43075300 & \\
\hline 2.35141000 & 0.38629900 & 2.590378 \\
\hline 4.34817800 & 2.56858500 & 00 \\
\hline 4.91808100 & 4.95968600 & -0.8 \\
\hline 3.28842700 & 6.59315300 & 0.12 \\
\hline 1.07169600 & & 0.92 \\
\hline 0.48032500 & 000 & \\
\hline 0.37374500 & & \\
\hline 0.41632300 & & \\
\hline-0.68280600 & .91690700 & -0.0940090 \\
\hline 3.61068100 & 3.67086500 & 0.683 \\
\hline 2.2772 & -3.2 & 1110 \\
\hline 1.67199500 & -3.10034000 & -0.0043010 \\
\hline 2.43905800 & -3.31410700 & -1.01813400 \\
\hline 3.77695600 & -3.70413000 & $-1.1250 / 50$ \\
\hline 4.36525200 & -3.88988100 & -0.47325400 \\
\hline 4.06619600 & -3.79773200 & 1.6616090 \\
\hline 1.70719700 & -3.08987300 & \\
\hline 1.95227800 & -3.19796700 & -2.78132 \\
\hline
\end{tabular}




\begin{tabular}{|c|c|c|c|c|c|c|c|}
\hline $\mathrm{H}$ & 4.35412100 & -3.87816200 & -2.63039800 & $\mathrm{C}$ & -4.56360900 & 3.86893800 & -0.37185100 \\
\hline $\mathrm{H}$ & 5.40375800 & -4.20206700 & -0.39621800 & $\mathrm{C}$ & -4.82382000 & 2.76213900 & -1.17937400 \\
\hline $\mathrm{C}$ & 0.18610200 & -2.81033100 & -0.84275100 & $\mathrm{C}$ & -4.16908600 & 1.55011900 & -0.94574000 \\
\hline $\mathrm{H}$ & 0.09765000 & -1.43750000 & -0.00705100 & $\mathrm{C}$ & -3.23801200 & 1.43071100 & 0.09615100 \\
\hline $\mathrm{O}$ & -0.22339100 & -2.57048000 & -2.02168900 & $\mathrm{C}$ & -3.01360800 & -2.38477500 & -1.14352600 \\
\hline $\mathrm{H}$ & -0.07601500 & -0.86738800 & -2.23362800 & $\mathrm{C}$ & -3.86838600 & -3.39480600 & -1.59511900 \\
\hline $\mathrm{C}$ & -0.72747200 & -3.62557500 & 0.07627600 & $\mathrm{C}$ & -5.18307800 & -3.46008900 & -1.13344800 \\
\hline $\mathrm{H}$ & -0.64505500 & -4.67967600 & -0.22424300 & $\mathrm{C}$ & -5.64790800 & -2.50933100 & -0.21929100 \\
\hline $\mathrm{H}$ & -0.45931300 & -3.54592800 & 1.13204600 & $\mathrm{C}$ & -4.79900200 & -1.49686600 & 0.22626300 \\
\hline \multirow[t]{2}{*}{$\mathrm{H}$} & -1.76231600 & -3.31021700 & -0.05387900 & $\mathrm{C}$ & -3.47075300 & -1.42591700 & -0.23033300 \\
\hline & & & & $\mathrm{C}$ & 2.63194300 & 2.79616600 & 0.07479900 \\
\hline $\mathbf{a} 2$ & & & & $\mathrm{C}$ & 3.27216400 & 3.97610900 & -0.30037900 \\
\hline $\mathrm{C}$ & -1.82537500 & 1.27782300 & -0.00003100 & $\mathrm{C}$ & 4.48527400 & 3.93009000 & -0.99221000 \\
\hline $\mathrm{C}$ & -0.43251100 & 1.19615200 & -0.00008600 & $\mathrm{C}$ & 5.05139200 & 2.69517500 & -1.30661600 \\
\hline $\mathrm{C}$ & 0.20534500 & -0.05370900 & -0.00007100 & $\mathrm{C}$ & 4.41951100 & 1.51070700 & -0.91868300 \\
\hline $\mathrm{C}$ & -0.57701400 & -1.21992200 & 0.00006000 & $\mathrm{C}$ & 3.20747500 & 1.54670300 & -0.21354500 \\
\hline $\mathrm{C}$ & -1.96611900 & -1.13860500 & 0.00005600 & $\mathrm{C}$ & 4.74604300 & -1.44049100 & 0.55078300 \\
\hline $\mathrm{C}$ & -2.59336500 & 0.11167400 & 0.00001100 & $\mathrm{C}$ & 5.59679900 & -2.51088200 & 0.27389500 \\
\hline $\mathrm{H}$ & -2.31035600 & 2.25015200 & -0.00002900 & $\mathrm{C}$ & 5.19504900 & -3.51097300 & -0.61616100 \\
\hline $\mathrm{H}$ & 0.15304900 & 2.11040600 & -0.00010500 & $\mathrm{C}$ & 3.94090400 & -3.43606000 & -1.22300300 \\
\hline $\mathrm{H}$ & -0.06737600 & -2.17792700 & 0.00012300 & $\mathrm{C}$ & 3.08508600 & -2.36825200 & -0.93990600 \\
\hline $\mathrm{H}$ & -2.56310600 & -2.04658100 & 0.00011500 & $\mathrm{C}$ & 3.47848300 & -1.35637600 & -0.05296400 \\
\hline $\mathrm{H}$ & -3.67828900 & 0.17609700 & 0.00004700 & $\mathrm{H}$ & -3.09004500 & 0.27731500 & 2.71483400 \\
\hline $\mathrm{C}$ & 1.69789200 & -0.20365500 & -0.00002700 & $\mathrm{H}$ & -2.73258600 & -1.42194600 & 2.40741200 \\
\hline $\mathrm{O}$ & 2.21267900 & -1.31183500 & -0.00010500 & $\mathrm{H}$ & -0.69698000 & 0.81727900 & 2.92948800 \\
\hline $\mathrm{C}$ & 2.56246400 & 1.04756200 & 0.00012200 & $\mathrm{H}$ & -1.01118200 & -0.62407100 & 3.92677800 \\
\hline $\mathrm{H}$ & 2.36288700 & 1.66587400 & -0.88339500 & $\mathrm{H}$ & 3.32701300 & -0.02101200 & 2.59341300 \\
\hline $\mathrm{H}$ & 2.36234600 & 1.66603600 & 0.88340700 & $\mathrm{H}$ & 1.95748300 & 1.09655100 & 2.45666500 \\
\hline \multirow[t]{2}{*}{$\mathrm{H}$} & 3.61150300 & 0.74670700 & 0.00047300 & $\mathrm{H}$ & 1.82506700 & -1.96983900 & 2.47749900 \\
\hline & & & & $\mathrm{H}$ & 1.31000500 & -0.92603400 & 3.80881100 \\
\hline 9 & & & & $\mathrm{H}$ & -2.21242100 & 2.50244000 & 1.66687300 \\
\hline $\mathrm{Ru}$ & -0.00319200 & -0.17534400 & -0.03428000 & $\mathrm{H}$ & -3.41375000 & 4.62272400 & 1.29193500 \\
\hline$P$ & -2.29265000 & -0.13310400 & 0.37515200 & $\mathrm{H}$ & -5.07600900 & 4.81032100 & -0.55162900 \\
\hline $\mathrm{P}$ & 2.29506000 & 0.02294500 & 0.30327900 & $\mathrm{H}$ & -5.53885300 & 2.83642800 & -1.99453300 \\
\hline $\mathrm{N}$ & 0.03792300 & -0.94696700 & 2.09512300 & $\mathrm{H}$ & -4.38927900 & 0.69636500 & -1.57862200 \\
\hline $\mathrm{C}$ & -2.36715500 & -0.40019000 & 2.24894800 & $\mathrm{H}$ & -1.98089200 & -2.33709900 & -1.47838400 \\
\hline $\mathrm{C}$ & -0.98779800 & -0.23540300 & 2.89571000 & $\mathrm{H}$ & -3.50270200 & -4.13101200 & -2.30620800 \\
\hline $\mathrm{C}$ & 2.32058300 & 0.10278600 & 2.17769100 & $\mathrm{H}$ & -5.84635300 & -4.24716600 & -1.48299900 \\
\hline $\mathrm{C}$ & 1.39528100 & -0.99102900 & 2.71468000 & $\mathrm{H}$ & -6.67190400 & -2.55542300 & 0.14267100 \\
\hline $\mathrm{C}$ & -2.96721600 & 2.55794400 & 0.88785200 & $\mathrm{H}$ & -5.17595500 & -0.75215000 & 0.92298900 \\
\hline $\mathrm{C}$ & -3.63182500 & 3.76262500 & 0.66398900 & $\mathrm{H}$ & 1.66326000 & 2.83356900 & 0.56483800 \\
\hline
\end{tabular}




\begin{tabular}{|c|c|c|c|c|c|c|c|}
\hline $\mathrm{H}$ & 2.81381600 & 4.93351000 & -0.06617400 & $\mathrm{C}$ & 3.20747500 & 1.54670300 & -0.21354500 \\
\hline $\mathrm{H}$ & 4.97831900 & 4.85059200 & -1.29375800 & $\mathrm{C}$ & 4.74604300 & -1.44049100 & 0.55078300 \\
\hline $\mathrm{H}$ & 5.98706300 & 2.64725100 & -1.85774900 & $\mathrm{C}$ & 5.59679900 & -2.51088200 & 0.27389500 \\
\hline $\mathrm{H}$ & 4.87491600 & 0.56013700 & -1.17384900 & $\mathrm{C}$ & 5.19504900 & -3.51097300 & -0.61616100 \\
\hline $\mathrm{H}$ & 5.08115300 & -0.66042800 & 1.22983400 & $\mathrm{C}$ & 3.94090400 & -3.43606000 & -1.22300300 \\
\hline $\mathrm{H}$ & 6.57251600 & -2.56349100 & 0.75021600 & $\mathrm{C}$ & 3.08508600 & -2.36825200 & -0.93990600 \\
\hline $\mathrm{H}$ & 5.85821600 & -4.34462000 & -0.83249900 & $\mathrm{C}$ & 3.47848300 & -1.35637600 & -0.05296400 \\
\hline $\mathrm{H}$ & 3.62217400 & -4.21202700 & -1.91410100 & $\mathrm{H}$ & -3.09004500 & 0.27731500 & 2.71483400 \\
\hline $\mathrm{H}$ & 2.09408500 & -2.31828000 & -1.38222000 & $\mathrm{H}$ & -2.73258600 & -1.42194600 & 2.40741200 \\
\hline $\mathrm{C}$ & -0.05722600 & 0.40502500 & -1.79160800 & $\mathrm{H}$ & -0.69698000 & 0.81727900 & 2.92948800 \\
\hline $\mathrm{O}$ & -0.09211400 & 0.75868600 & -2.90080100 & $\mathrm{H}$ & -1.01118200 & -0.62407100 & 3.92677800 \\
\hline $\mathrm{H}$ & 0.04497800 & -1.80487500 & -0.51732800 & $\mathrm{H}$ & 3.32701300 & -0.02101200 & 2.59341300 \\
\hline $\mathrm{H}$ & -0.04896000 & 1.39819900 & 0.62550000 & $\mathrm{H}$ & 1.95748300 & 1.09655100 & 2.45666500 \\
\hline \multirow[t]{2}{*}{$\mathrm{H}$} & -0.27147800 & -1.90921700 & 1.97069500 & $\mathrm{H}$ & 1.82506700 & -1.96983900 & 2.47749900 \\
\hline & & & & $\mathrm{H}$ & 1.31000500 & -0.92603400 & 3.80881100 \\
\hline 9-D & & & & $\mathrm{H}$ & -2.21242100 & 2.50244000 & 1.66687300 \\
\hline $\mathrm{Ru}$ & -0.00319200 & -0.17534400 & -0.03428000 & $\mathrm{H}$ & -3.41375000 & 4.62272400 & 1.29193500 \\
\hline $\mathrm{P}$ & -2.29265000 & -0.13310400 & 0.37515200 & $\mathrm{H}$ & -5.07600900 & 4.81032100 & -0.55162900 \\
\hline $\mathrm{P}$ & 2.29506000 & 0.02294500 & 0.30327900 & $\mathrm{H}$ & -5.53885300 & 2.83642800 & -1.99453300 \\
\hline $\mathrm{N}$ & 0.03792300 & -0.94696700 & 2.09512300 & $\mathrm{H}$ & -4.38927900 & 0.69636500 & -1.57862200 \\
\hline $\mathrm{C}$ & -2.36715500 & -0.40019000 & 2.24894800 & $\mathrm{H}$ & -1.98089200 & -2.33709900 & -1.47838400 \\
\hline $\mathrm{C}$ & -0.98779800 & -0.23540300 & 2.89571000 & $\mathrm{H}$ & -3.50270200 & -4.13101200 & -2.30620800 \\
\hline $\mathrm{C}$ & 2.32058300 & 0.10278600 & 2.17769100 & $\mathrm{H}$ & -5.84635300 & -4.24716600 & -1.48299900 \\
\hline $\mathrm{C}$ & 1.39528100 & -0.99102900 & 2.71468000 & $\mathrm{H}$ & -6.67190400 & -2.55542300 & 0.14267100 \\
\hline $\mathrm{C}$ & -2.96721600 & 2.55794400 & 0.88785200 & $\mathrm{H}$ & -5.17595500 & -0.75215000 & 0.92298900 \\
\hline $\mathrm{C}$ & -3.63182500 & 3.76262500 & 0.66398900 & $\mathrm{H}$ & 1.66326000 & 2.83356900 & 0.56483800 \\
\hline $\mathrm{C}$ & -4.56360900 & 3.86893800 & -0.37185100 & $\mathrm{H}$ & 2.81381600 & 4.93351000 & -0.06617400 \\
\hline $\mathrm{C}$ & -4.82382000 & 2.76213900 & -1.17937400 & $\mathrm{H}$ & 4.97831900 & 4.85059200 & -1.29375800 \\
\hline $\mathrm{C}$ & -4.16908600 & 1.55011900 & -0.94574000 & $\mathrm{H}$ & 5.98706300 & 2.64725100 & -1.85774900 \\
\hline $\mathrm{C}$ & -3.23801200 & 1.43071100 & 0.09615100 & $\mathrm{H}$ & 4.87491600 & 0.56013700 & -1.17384900 \\
\hline $\mathrm{C}$ & -3.01360800 & -2.38477500 & -1.14352600 & $\mathrm{H}$ & 5.08115300 & -0.66042800 & 1.22983400 \\
\hline $\mathrm{C}$ & -3.86838600 & -3.39480600 & -1.59511900 & $\mathrm{H}$ & 6.57251600 & -2.56349100 & 0.75021600 \\
\hline $\mathrm{C}$ & -5.18307800 & -3.46008900 & -1.13344800 & $\mathrm{H}$ & 5.85821600 & -4.34462000 & -0.83249900 \\
\hline $\mathrm{C}$ & -5.64790800 & -2.50933100 & -0.21929100 & $\mathrm{H}$ & 3.62217400 & -4.21202700 & -1.91410100 \\
\hline $\mathrm{C}$ & -4.79900200 & -1.49686600 & 0.22626300 & $\mathrm{H}$ & 2.09408500 & -2.31828000 & -1.38222000 \\
\hline $\mathrm{C}$ & -3.47075300 & -1.42591700 & -0.23033300 & $\mathrm{C}$ & -0.05722600 & 0.40502500 & -1.79160800 \\
\hline $\mathrm{C}$ & 2.63194300 & 2.79616600 & 0.07479900 & $\mathrm{O}$ & -0.09211400 & 0.75868600 & -2.90080100 \\
\hline $\mathrm{C}$ & 3.27216400 & 3.97610900 & -0.30037900 & $\mathrm{D}$ & 0.04497800 & -1.80487500 & -0.51732800 \\
\hline $\mathrm{C}$ & 4.48527400 & 3.93009000 & -0.99221000 & $\mathrm{H}$ & -0.04896000 & 1.39819900 & 0.62550000 \\
\hline $\mathrm{C}$ & 5.05139200 & 2.69517500 & -1.30661600 & $\mathrm{H}$ & -0.27147800 & -1.90921700 & 1.97069500 \\
\hline $\mathrm{C}$ & 4.41951100 & 1.51070700 & -0.91868300 & & & & \\
\hline
\end{tabular}


b1

C

H

$\mathrm{H}$

C

H

$\mathrm{H}$

C

$\mathrm{H}$

C

$\mathrm{H}$

$\mathrm{H}$

C

$\mathrm{H}$

C

$\mathrm{H}$

$\mathrm{H}$

$\mathrm{H}$

O

$\mathrm{H}$

10

$\mathrm{Ru}$

P

P

$\mathrm{N}$

C

C

C

C

C

C

C

C

C

C

C

C

C

C

C

$\begin{array}{rrr}0.08188200 & 0.36277500 & 1.26514700 \\ 1.12162200 & 0.72653500 & 1.30923400 \\ -0.43041300 & 0.76064400 & 2.14921600 \\ 0.08188200 & -1.17534300 & 1.26927000 \\ 0.59340800 & -1.54970700 & 2.16483400 \\ -0.95562300 & -1.53565800 & 1.33128700 \\ -0.57716600 & 0.92900600 & 0.00000000 \\ -1.63841000 & 0.64622200 & 0.00000000 \\ 0.08188200 & 0.36277500 & -1.26514700 \\ 1.12162200 & 0.72653500 & -1.30923400 \\ -0.43041300 & 0.76064400 & -2.14921600 \\ 0.73792400 & -1.74179600 & 0.00000000 \\ 0.68076600 & -2.83772500 & 0.00000000 \\ 0.08188200 & -1.17534300 & -1.26927000 \\ -0.95562300 & -1.53565800 & -1.33128700 \\ 0.59340800 & -1.54970700 & -2.16483400 \\ 1.80763800 & -1.48429400 & 0.00000000 \\ -0.59571400 & 2.35557300 & 0.00000000 \\ 0.32802100 & 2.65514200 & 0.00000000\end{array}$

C

C

C

C

C

C

C

C

C

C

C

C

C

$\mathrm{H}$

$\mathrm{H}$

H

$\mathrm{H}$

$\mathrm{H}$

$\mathrm{H}$

$\mathrm{H}$

$\mathrm{H}$

$\mathrm{H}$

$\mathrm{H}$

H

$\mathrm{H}$

$\mathrm{H}$

$\mathrm{H}$

H

H

$\mathrm{H}$

$\mathrm{H}$

$\mathrm{H}$

H

$\mathrm{H}$

$\mathrm{H}$

$\mathrm{H}$

$\mathrm{H}$

$\mathrm{H}$

$\mathrm{H}$

$\mathrm{H}$

$\mathrm{H}$

\begin{tabular}{|c|c|c|}
\hline 3.28470700 & 2.11500500 & -0.64 \\
\hline-2.90591400 & 1.36801000 & 1.7376950 \\
\hline 3.60625800 & 1.98131000 & 2.7758950 \\
\hline 1.74675400 & 1.37716900 & 3.3120470 \\
\hline-5.18376800 & 0.15405900 & 2.8038710 \\
\hline-4.49090500 & -0.46201800 & 1.75832700 \\
\hline-3.35162700 & 0.14205800 & 1.2093600 \\
\hline-4.52347700 & -2.00976400 & -1.30657700 \\
\hline-5.22532600 & -3.15411600 & -1.68591200 \\
\hline-4.70238600 & -4.41983200 & -1.40908300 \\
\hline 3.47765600 & -4.53494700 & -0.75047600 \\
\hline-2.77234900 & -3.38953500 & -0.37476200 \\
\hline-3.28539300 & -2.11341200 & -0.64949700 \\
\hline 2.51620200 & 1.57852200 & -1.0995160 \\
\hline 3.37151300 & 0.43023900 & -2.1412360 \\
\hline 1.17166900 & 1.30516900 & -3.1140380 \\
\hline 1.25669400 & 0.45483700 & -3.0208820 \\
\hline-3.37168100 & 0.43291700 & -2.1401360 \\
\hline-2.51495900 & 1.58005700 & -1.0982620 \\
\hline-1.25771300 & -0.45365600 & -3.0207080 \\
\hline-1.17151800 & 1.30631900 & -3.1134850 \\
\hline 2.00736400 & 1.84029000 & 1.3436210 \\
\hline 3.25273800 & 2.92875100 & \\
\hline 5.28626800 & & \\
\hline 6.06556200 & -0.32670000 & 3.2190580 \\
\hline 4.84224100 & 9400 & 1.37 \\
\hline 1.81357500 & -3.479 & \\
\hline 3.06410200 & -5.51786400 & -0.5286160 \\
\hline 5.24689000 & -5.31405300 & 1.70 \\
\hline 6.17883800 & $0.00 \mathrm{~s}$ & \\
\hline 4.95080000 & -1.03564700 & 15157160 \\
\hline-2.00817800 & 1.84151600 & 1.3437630 \\
\hline-3.25352600 & 2.92955200 & 3.173166 \\
\hline 5.28653100 & 85 & 4.1. \\
\hline 6.06528900 & -0.320 & 3.220 \\
\hline-4.84194900 & -1.41441600 & $1.3 / 45200$ \\
\hline-4.95114700 & -1.03298900 & -1.51552500 \\
\hline-6.17986500 & -3.05736700 & -2.19647800 \\
\hline-5.24866700 & -5.31117400 & -1.70608800 \\
\hline 3.06593600 & -5.51641300 & -0.53113900 \\
\hline 4100 & 7700 & 0.12792 \\
\hline
\end{tabular}




\begin{tabular}{|c|c|c|c|c|c|c|c|}
\hline $\mathrm{C}$ & -0.00009200 & -1.61328900 & 1.83552500 & $\mathrm{C}$ & 4.81834600 & -4.23948600 & -0.36201900 \\
\hline $\mathrm{O}$ & -0.00002700 & -2.16959700 & 2.85883900 & $\mathrm{C}$ & 5.13393800 & -3.23190700 & -1.27456800 \\
\hline $\mathrm{H}$ & -0.00047800 & -2.16511800 & -0.49764600 & $\mathrm{C}$ & 4.42752100 & -2.02696400 & -1.26032700 \\
\hline $\mathrm{C}$ & -1.26302000 & 4.39946000 & -1.10614400 & $\mathrm{C}$ & 3.38932900 & -1.81499000 & -0.33930800 \\
\hline $\mathrm{H}$ & -1.30651700 & 3.72918300 & -1.97851300 & $\mathrm{C}$ & -3.17913700 & 1.01977900 & 1.64155000 \\
\hline $\mathrm{H}$ & -2.15034200 & 4.18918200 & -0.49605800 & C & -4.16571200 & 1.68452500 & 2.37403100 \\
\hline $\mathrm{C}$ & -1.26597700 & 5.86081000 & -1.58635600 & C & -5.50686000 & 1.32915500 & 2.22465300 \\
\hline $\mathrm{H}$ & -2.16152600 & 6.05789300 & -2.18922700 & $\mathrm{C}$ & -5.86095300 & 0.30433900 & 1.34291100 \\
\hline $\mathrm{H}$ & -1.32662200 & 6.52786100 & -0.71355600 & C & -4.87715600 & -0.36145100 & 0.61197400 \\
\hline $\mathrm{C}$ & 0.00000000 & 4.06671500 & -0.29551100 & $\mathrm{C}$ & -3.52586000 & -0.00577600 & 0.74929400 \\
\hline $\mathrm{H}$ & -0.00057300 & 4.69321800 & 0.60822300 & $\mathrm{C}$ & -3.46804600 & -3.18825700 & -1.23250600 \\
\hline $\mathrm{C}$ & 1.26491800 & 4.39819800 & -1.10368500 & $\mathrm{C}$ & -3.84312200 & -4.53260800 & -1.15363700 \\
\hline $\mathrm{H}$ & 1.30942100 & 3.72789500 & -1.97598800 & $\mathrm{C}$ & -3.46498200 & -5.30259500 & -0.05433100 \\
\hline $\mathrm{H}$ & 2.15085700 & 4.18701900 & -0.49190200 & $\mathrm{C}$ & -2.70242700 & -4.72504500 & 0.96434200 \\
\hline $\mathrm{C}$ & 0.00309200 & 6.19248600 & -2.38685000 & $\mathrm{C}$ & -2.31779100 & -3.38801400 & 0.88004500 \\
\hline $\mathrm{H}$ & 0.00390600 & 7.25095600 & -2.67713800 & $\mathrm{C}$ & -2.70231700 & -2.59801400 & -0.21676500 \\
\hline $\mathrm{C}$ & 1.27026700 & 5.85954300 & -1.58389000 & $\mathrm{H}$ & 2.32106200 & 1.56553700 & -1.81416500 \\
\hline $\mathrm{H}$ & 1.32988200 & 6.52652900 & -0.71097100 & $\mathrm{H}$ & 3.40679100 & 0.33803800 & -2.48584400 \\
\hline $\mathrm{H}$ & 2.16718600 & 6.05572500 & -2.18501500 & $\mathrm{H}$ & 1.20659200 & 0.44491000 & -3.72330400 \\
\hline $\mathrm{H}$ & 0.00370900 & 5.61120100 & -3.32098900 & $\mathrm{H}$ & 1.45096000 & -1.14198900 & -2.98098800 \\
\hline $\mathrm{O}$ & -0.00114400 & 2.72823400 & 0.18688600 & $\mathrm{H}$ & -3.28410500 & -0.64087900 & -2.46689400 \\
\hline \multirow[t]{2}{*}{$\mathrm{H}$} & 0.00010500 & 2.06276500 & -0.55908200 & $\mathrm{H}$ & -2.62477800 & 0.87222300 & -1.85245200 \\
\hline & & & & $\mathrm{H}$ & -1.00208700 & -1.47881000 & -2.98198000 \\
\hline \multirow{2}{*}{\multicolumn{4}{|c|}{$\begin{array}{l}\text { TS5 } \\
\text { imaginary frequency }=-1068.60 \mathrm{~cm}^{-1}\end{array}$}} & $\mathrm{H}$ & -1.20949800 & 0.11509200 & -3.71836200 \\
\hline & & & & $\mathrm{H}$ & 1.86385300 & 2.22459000 & 1.09628800 \\
\hline $\mathrm{Ru}$ & 0.10576300 & -0.40801100 & 0.13443400 & $\mathrm{H}$ & 3.20346500 & 3.82351100 & 2.45226700 \\
\hline $\mathrm{P}$ & 2.43506000 & -0.23921800 & -0.24570600 & $\mathrm{H}$ & 5.61271000 & 3.37442500 & 2.88196600 \\
\hline $\mathrm{P}$ & -2.18307500 & -0.82608800 & -0.21801700 & $\mathrm{H}$ & 6.66945600 & 1.32254200 & 1.95725000 \\
\hline $\mathrm{N}$ & 0.02835400 & 0.08790400 & -1.99578600 & $\mathrm{H}$ & 5.33922800 & -0.26374600 & 0.61429600 \\
\hline $\mathrm{C}$ & 2.45791700 & 0.48980900 & -1.96174100 & $\mathrm{H}$ & 2.26685900 & -2.69627900 & 1.27597300 \\
\hline $\mathrm{C}$ & 1.27368900 & -0.07784800 & -2.75260900 & $\mathrm{H}$ & 3.53734200 & -4.81905400 & 1.27464300 \\
\hline $\mathrm{C}$ & -2.42212800 & -0.19773400 & -1.95761900 & $\mathrm{H}$ & 5.36744300 & -5.17728600 & -0.37359300 \\
\hline $\mathrm{C}$ & -1.12591400 & -0.40809200 & -2.74907300 & $\mathrm{H}$ & 5.92997900 & -3.38079100 & -1.99947100 \\
\hline $\mathrm{C}$ & 2.91632400 & 2.03751500 & 1.28742700 & $\mathrm{H}$ & 4.69729300 & -1.25305600 & -1.97258600 \\
\hline $\mathrm{C}$ & 3.67332700 & 2.93240600 & 2.04402000 & $\mathrm{H}$ & -2.13399700 & 1.29179800 & 1.75809900 \\
\hline $\mathrm{C}$ & 5.02591200 & 2.68020700 & 2.28601400 & $\mathrm{H}$ & -3.88364000 & 2.47513700 & 3.06418700 \\
\hline $\mathrm{C}$ & 5.61937300 & 1.52882200 & 1.76654200 & $\mathrm{H}$ & -6.27433600 & 1.84336100 & 2.79719000 \\
\hline $\mathrm{C}$ & 4.86700800 & 0.63251100 & 1.00403100 & $\mathrm{H}$ & -6.90368500 & 0.01944200 & 1.22895300 \\
\hline C & 3.51013400 & 0.88170700 & 0.75201800 & $\mathrm{H}$ & -5.16151100 & -1.16985500 & -0.05600500 \\
\hline C & 3.07892900 & -2.83900900 & 0.57021200 & $\mathrm{H}$ & -3.77693000 & -2.61090600 & -2.09824100 \\
\hline C & 3.79142100 & -4.03832500 & 0.56272100 & $\mathrm{H}$ & -4.43049100 & -4.97443000 & -1.95438500 \\
\hline
\end{tabular}




\begin{tabular}{|c|c|c|c|c|c|c|c|}
\hline $\mathrm{H}$ & -3.75734700 & -6.34745600 & 0.00714200 & $\mathrm{C}$ & -3.14292200 & -2.61824100 & -0.96049200 \\
\hline $\mathrm{H}$ & -2.39831800 & -5.31896600 & 1.82211200 & $\mathrm{C}$ & -3.84715700 & -3.80644800 & -1.15046300 \\
\hline $\mathrm{H}$ & -1.70435300 & -2.95400300 & 1.66404400 & $\mathrm{C}$ & -4.79400900 & -4.22056100 & -0.21021500 \\
\hline $\mathrm{C}$ & 0.16139800 & -0.70145400 & 1.97712400 & $\mathrm{C}$ & -5.03573600 & -3.43617000 & 0.91751600 \\
\hline $\mathrm{O}$ & 0.19926000 & -0.93250400 & 3.11957600 & $\mathrm{C}$ & -4.33907600 & -2.23860700 & 1.10136500 \\
\hline $\mathrm{H}$ & 0.27839500 & -1.96937600 & -0.03957500 & $\mathrm{C}$ & -3.38449100 & -1.81305600 & 0.16500200 \\
\hline $\mathrm{C}$ & -1.87646400 & 3.47220500 & -0.99881500 & $\mathrm{C}$ & 3.29345700 & 1.07200600 & -1.45681600 \\
\hline $\mathrm{H}$ & -1.81903200 & 3.19410200 & -2.06297700 & $\mathrm{C}$ & 4.32847300 & 1.78699900 & -2.06481000 \\
\hline $\mathrm{H}$ & -2.58362600 & 2.78493200 & -0.52014700 & $\mathrm{C}$ & 5.65392700 & 1.53979200 & -1.70689600 \\
\hline $\mathrm{C}$ & -2.38259900 & 4.92015300 & -0.87827600 & $\mathrm{C}$ & 5.94539700 & 0.57032500 & -0.74302600 \\
\hline $\mathrm{H}$ & -3.36560000 & 5.01712100 & -1.35731200 & $\mathrm{C}$ & 4.91376400 & -0.14917900 & -0.14139200 \\
\hline $\mathrm{H}$ & -2.53179100 & 5.16187100 & 0.18475500 & $\mathrm{C}$ & 3.57546900 & 0.09912700 & -0.48739000 \\
\hline $\mathrm{C}$ & -0.48105300 & 3.29200100 & -0.37320900 & $\mathrm{C}$ & 3.49950500 & -3.30486400 & 0.89009800 \\
\hline $\mathrm{H}$ & -0.56890100 & 3.52846900 & 0.70221900 & $\mathrm{C}$ & 3.87311000 & -4.62114700 & 0.60575300 \\
\hline $\mathrm{C}$ & 0.50457200 & 4.30263300 & -0.99352800 & $\mathrm{C}$ & 3.43939900 & -5.23422000 & -0.56940500 \\
\hline $\mathrm{H}$ & 0.63529500 & 4.04305500 & -2.05545400 & $\mathrm{C}$ & 2.62287500 & -4.52771600 & -1.45589400 \\
\hline $\mathrm{H}$ & 1.48597700 & 4.18651800 & -0.51759300 & $\mathrm{C}$ & 2.23834500 & -3.21957000 & -1.16504400 \\
\hline $\mathrm{C}$ & -1.38888900 & 5.92097000 & -1.48705200 & $\mathrm{C}$ & 2.67790500 & -2.58619100 & 0.00915400 \\
\hline $\mathrm{H}$ & -1.74802900 & 6.94875800 & -1.34542700 & $\mathrm{H}$ & -2.25235800 & 1.28739300 & 2.13692600 \\
\hline $\mathrm{C}$ & 0.01006900 & 5.75339100 & -0.87481600 & $\mathrm{H}$ & -3.45886600 & 0.06657600 & 2.59622100 \\
\hline $\mathrm{H}$ & -0.02590800 & 6.03860500 & 0.18749800 & $\mathrm{H}$ & -1.33681500 & -0.23739800 & 3.91081200 \\
\hline $\mathrm{H}$ & 0.72220400 & 6.43764800 & -1.35433500 & $\mathrm{H}$ & -1.56908400 & -1.63051300 & 2.83756100 \\
\hline $\mathrm{H}$ & -1.33002500 & 5.75643300 & -2.57346900 & $\mathrm{H}$ & 3.23322000 & -1.02200700 & 2.55383800 \\
\hline $\mathrm{O}$ & 0.02041200 & 1.97909200 & -0.48321400 & $\mathrm{H}$ & 2.51914700 & 0.55525400 & 2.21677000 \\
\hline \multirow[t]{2}{*}{$\mathrm{H}$} & -0.07291200 & 1.30545200 & -1.45618000 & $\mathrm{H}$ & 0.93668700 & -2.01899200 & 2.79845900 \\
\hline & & & & $\mathrm{H}$ & 1.13690700 & -0.64319400 & 3.89933100 \\
\hline 11 & & & & $\mathrm{H}$ & -1.85443300 & 2.10337300 & -1.26196600 \\
\hline $\mathrm{Ru}$ & -0.10858700 & -0.33261100 & -0.04134500 & $\mathrm{H}$ & -3.24820100 & 3.80467000 & -2.42451600 \\
\hline $\mathrm{P}$ & -2.44269400 & -0.23278800 & 0.31454100 & $\mathrm{H}$ & -5.72904600 & 3.66750700 & -2.30615400 \\
\hline $\mathrm{P}$ & 2.17246200 & -0.82690200 & 0.27826600 & $\mathrm{H}$ & -6.80606400 & 1.81647100 & -1.04242700 \\
\hline $\mathrm{N}$ & -0.08400400 & -0.29202200 & 2.19185900 & $\mathrm{H}$ & -5.42078400 & 0.10849400 & 0.07839500 \\
\hline $\mathrm{C}$ & -2.48377500 & 0.21687400 & 2.12255500 & $\mathrm{H}$ & -2.38826500 & -2.31634700 & -1.68024800 \\
\hline $\mathrm{C}$ & -1.37697700 & -0.55107300 & 2.85537400 & $\mathrm{H}$ & -3.64857800 & -4.41468400 & -2.02885300 \\
\hline $\mathrm{C}$ & 2.36373700 & -0.52422600 & 2.11336700 & $\mathrm{H}$ & -5.33598800 & -5.15150000 & -0.35392200 \\
\hline $\mathrm{C}$ & 1.07538500 & -0.93219100 & 2.83777100 & $\mathrm{H}$ & -5.76647000 & -3.75279600 & 1.65726500 \\
\hline $\mathrm{C}$ & -2.93673200 & 2.04357800 & -1.22726000 & $\mathrm{H}$ & -4.54917200 & -1.64260300 & 1.98453700 \\
\hline $\mathrm{C}$ & -3.72459500 & 3.00064300 & -1.87001200 & $\mathrm{H}$ & 2.26136800 & 1.27240700 & -1.72709700 \\
\hline $\mathrm{C}$ & -5.11673200 & 2.92381500 & -1.80276400 & $\mathrm{H}$ & 4.09549700 & 2.53707400 & -2.81560900 \\
\hline $\mathrm{C}$ & -5.72238700 & 1.88457800 & -1.09231600 & $\mathrm{H}$ & 6.45924500 & 2.09688500 & -2.17830100 \\
\hline $\mathrm{C}$ & -4.93835600 & 0.92433000 & -0.45214400 & $\mathrm{H}$ & 6.97696300 & 0.37091900 & -0.46471200 \\
\hline $\mathrm{C}$ & -3.53784900 & 0.99882400 & -0.50969800 & $\mathrm{H}$ & 5.15569000 & -0.91616600 & 0.58933300 \\
\hline
\end{tabular}




\begin{tabular}{|c|c|c|c|c|c|c|c|}
\hline $\mathrm{H}$ & 3.85536100 & -2.85187900 & 1.81056900 & $\mathrm{C}$ & 4.34740200 & 1.38634700 & -0.15011100 \\
\hline $\mathrm{H}$ & 4.50319600 & -5.16431200 & 1.30535700 & $\mathrm{C}$ & 3.48451300 & 0.56504700 & 0.59182200 \\
\hline $\mathrm{H}$ & 3.73099000 & -6.25729600 & -0.79139900 & $\mathrm{C}$ & 2.46808700 & -3.41313300 & 0.33187000 \\
\hline $\mathrm{H}$ & 2.27548600 & -4.99883900 & -2.37150300 & $\mathrm{C}$ & 3.12032800 & -4.64284600 & 0.45399800 \\
\hline $\mathrm{H}$ & 1.58009200 & -2.68746300 & -1.84472000 & $\mathrm{C}$ & 4.50287400 & -4.72304400 & 0.28788100 \\
\hline $\mathrm{C}$ & -0.12853600 & -0.31200500 & -1.89068400 & $\mathrm{C}$ & 5.23460400 & -3.56757000 & 0.00018700 \\
\hline $\mathrm{O}$ & -0.14502400 & -0.33925700 & -3.05762100 & $\mathrm{C}$ & 4.58457300 & -2.34033700 & -0.12223100 \\
\hline $\mathrm{H}$ & -0.27650800 & -1.93035000 & -0.13571600 & $\mathrm{C}$ & 3.19132200 & -2.24931900 & 0.03856300 \\
\hline $\mathrm{C}$ & 1.81539400 & 3.28540200 & 1.05428900 & $\mathrm{C}$ & -3.43561800 & 0.86111200 & 1.91626900 \\
\hline $\mathrm{H}$ & 1.63416600 & 3.24757700 & 2.14059900 & $\mathrm{C}$ & -4.32887200 & 1.74500000 & 2.52250400 \\
\hline $\mathrm{H}$ & 2.51714900 & 2.47796000 & 0.81617500 & $\mathrm{C}$ & -5.35089000 & 2.33385200 & 1.77493000 \\
\hline $\mathrm{C}$ & 2.43402400 & 4.64340400 & 0.68278300 & $\mathrm{C}$ & -5.47892700 & 2.03163700 & 0.41830900 \\
\hline $\mathrm{H}$ & 3.37690400 & 4.79574900 & 1.22561000 & $\mathrm{C}$ & -4.59010600 & 1.14182200 & -0.18875200 \\
\hline $\mathrm{H}$ & 2.69229700 & 4.64097100 & -0.38706300 & $\mathrm{C}$ & -3.55670900 & 0.54610400 & 0.55294300 \\
\hline $\mathrm{C}$ & 0.46587200 & 3.02160800 & 0.34709700 & $\mathrm{C}$ & -4.52362700 & -2.42227600 & 0.32922100 \\
\hline $\mathrm{H}$ & 0.66509100 & 3.03910400 & -0.74593400 & $\mathrm{C}$ & -5.10412800 & -3.68887600 & 0.42257900 \\
\hline $\mathrm{C}$ & -0.48636900 & 4.20182600 & 0.65132100 & $\mathrm{C}$ & -4.33878800 & -4.83229200 & 0.18884300 \\
\hline $\mathrm{H}$ & -0.70958600 & 4.17771600 & 1.72922900 & $\mathrm{C}$ & -2.98608400 & -4.70443400 & -0.13324000 \\
\hline $\mathrm{H}$ & -1.43935700 & 4.04148100 & 0.13384800 & $\mathrm{C}$ & -2.40127800 & -3.44036200 & -0.21601200 \\
\hline $\mathrm{C}$ & 1.46374300 & 5.80046600 & 0.96396500 & $\mathrm{C}$ & -3.16507300 & -2.28295800 & 0.00710900 \\
\hline $\mathrm{H}$ & 1.90091200 & 6.75467100 & 0.63989300 & $\mathrm{H}$ & 2.38500000 & 0.75933000 & -2.17502500 \\
\hline $\mathrm{C}$ & 0.11080400 & 5.56754200 & 0.27483500 & $\mathrm{H}$ & 3.36294700 & -0.71378000 & -2.45076300 \\
\hline $\mathrm{H}$ & 0.25213900 & 5.61400500 & -0.81598500 & $\mathrm{H}$ & 1.13011500 & -0.59696600 & -3.71294400 \\
\hline $\mathrm{H}$ & -0.58968400 & 6.37475200 & 0.52910700 & $\mathrm{H}$ & 1.30453700 & -2.04814000 & -2.71983700 \\
\hline $\mathrm{H}$ & 1.30526000 & 5.88140700 & 2.05006600 & $\mathrm{H}$ & -3.38362200 & -0.72924100 & -2.47142800 \\
\hline $\mathrm{O}$ & -0.11597400 & 1.81707100 & 0.73277300 & $\mathrm{H}$ & -2.45653200 & 0.76333100 & -2.17785000 \\
\hline \multirow[t]{2}{*}{$\mathrm{H}$} & 0.05350200 & 0.74176900 & 2.10593400 & $\mathrm{H}$ & -1.33035300 & -2.02015700 & -2.77198300 \\
\hline & & & & $\mathrm{H}$ & -1.14136300 & -0.54586500 & -3.72293700 \\
\hline 12 & & & & $\mathrm{H}$ & 2.82864000 & 0.04496100 & 2.58607800 \\
\hline $\mathrm{Ru}$ & -0.01904800 & -0.49513500 & 0.13480500 & $\mathrm{H}$ & 4.35737600 & 1.61134100 & 3.72056300 \\
\hline $\mathrm{P}$ & 2.32147500 & -0.63097900 & -0.19066500 & $\mathrm{H}$ & 5.88261800 & 3.05914000 & 2.38887700 \\
\hline $\mathrm{P}$ & -2.35661100 & -0.63132500 & -0.19916000 & $\mathrm{H}$ & 5.86654900 & 2.91081500 & -0.09381500 \\
\hline $\mathrm{N}$ & -0.01437500 & -0.56729700 & -1.93147500 & $\mathrm{H}$ & 4.35539300 & 1.33739300 & -1.23431200 \\
\hline $\mathrm{C}$ & 2.43368500 & -0.32392400 & -2.02125600 & $\mathrm{H}$ & 1.39382100 & -3.34786400 & 0.47229900 \\
\hline $\mathrm{C}$ & 1.19527200 & -0.94967000 & -2.66990000 & $\mathrm{H}$ & 2.54544200 & -5.53593200 & 0.68473800 \\
\hline $\mathrm{C}$ & -2.46384000 & -0.32338600 & -2.03630800 & $\mathrm{H}$ & 5.01065700 & -5.67886700 & 0.38673800 \\
\hline $\mathrm{C}$ & -1.21633600 & -0.92381100 & -2.68899300 & $\mathrm{H}$ & 6.31302800 & -3.62142100 & -0.12404800 \\
\hline $\mathrm{C}$ & 3.49933900 & 0.66041500 & 1.99366900 & $\mathrm{H}$ & 5.16616700 & -1.44689100 & -0.33254300 \\
\hline $\mathrm{C}$ & 4.36052800 & 1.54898300 & 2.63575800 & $\mathrm{H}$ & -2.63456800 & 0.42526800 & 2.50432800 \\
\hline $\mathrm{C}$ & 5.21602500 & 2.36223100 & 1.88805100 & $\mathrm{H}$ & -4.21888900 & 1.97976900 & 3.57765900 \\
\hline $\mathrm{C}$ & 5.20649800 & 2.27987200 & 0.49557100 & $\mathrm{H}$ & -6.04131100 & 3.02822200 & 2.24612700 \\
\hline
\end{tabular}




\begin{tabular}{|c|c|c|c|c|c|c|c|}
\hline $\mathrm{H}$ & -6.27000900 & 2.48783100 & -0.17089900 & $\mathrm{C}$ & 4.47390300 & -1.95463200 & -2.19771200 \\
\hline $\mathrm{H}$ & -4.70899800 & 0.91405400 & -1.24366700 & $\mathrm{C}$ & 5.44740600 & -2.52989500 & -1.37748000 \\
\hline $\mathrm{H}$ & -5.13206900 & -1.54269300 & 0.51388600 & $\mathrm{C}$ & 5.48037300 & -2.21505500 & -0.01864200 \\
\hline $\mathrm{H}$ & -6.15598300 & -3.77969700 & 0.68089500 & $\mathrm{C}$ & 4.54871400 & -1.32306900 & 0.51848200 \\
\hline $\mathrm{H}$ & -4.79209000 & -5.81727700 & 0.26258400 & C & 3.56682500 & -0.73953700 & -0.29664100 \\
\hline $\mathrm{H}$ & -2.38141200 & -5.58989600 & -0.31132900 & $\mathrm{C}$ & 2.52486300 & 3.08862400 & -0.80105500 \\
\hline $\mathrm{H}$ & -1.34352200 & -3.34489000 & -0.44216000 & $\mathrm{C}$ & 3.18015500 & 4.28296400 & -1.11426800 \\
\hline C & -0.00009800 & 0.10545600 & 1.91290300 & C & 4.50419500 & 4.48113200 & -0.72431400 \\
\hline $\mathrm{O}$ & 0.01856000 & 0.50331000 & 3.00977600 & $\mathrm{C}$ & 5.17802500 & 3.47536700 & -0.02550200 \\
\hline $\mathrm{H}$ & -0.04873600 & -1.90828200 & 0.82431400 & $\mathrm{C}$ & 4.52738400 & 2.28096400 & 0.27899000 \\
\hline $\mathrm{C}$ & -1.16831500 & 3.79423600 & -1.38732500 & $\mathrm{C}$ & 3.18832300 & 2.07618100 & -0.09699500 \\
\hline $\mathrm{H}$ & -1.25418800 & 4.29883800 & -2.36032900 & $\mathrm{C}$ & -3.50861100 & -1.10060000 & -1.67398400 \\
\hline $\mathrm{H}$ & -1.94738000 & 3.02086900 & -1.36057700 & $\mathrm{C}$ & -4.43976900 & -1.98598700 & -2.21667800 \\
\hline $\mathrm{C}$ & -1.38313500 & 4.80400000 & -0.24800200 & $\mathrm{C}$ & -5.43264500 & -2.54411100 & -1.40816300 \\
\hline $\mathrm{H}$ & -2.36614200 & 5.28216100 & -0.34807700 & C & -5.49070500 & -2.21034700 & -0.05452900 \\
\hline $\mathrm{H}$ & -1.39531100 & 4.26976300 & 0.71369500 & $\mathrm{C}$ & -4.56442700 & -1.31678000 & 0.48858300 \\
\hline $\mathrm{C}$ & 0.21853800 & 3.13357300 & -1.32252300 & $\mathrm{C}$ & -3.56324400 & -0.74985200 & -0.31521500 \\
\hline $\mathrm{H}$ & 0.27752400 & 2.53797200 & -0.39106600 & $\mathrm{C}$ & -4.53017200 & 2.27351700 & 0.29209100 \\
\hline $\mathrm{C}$ & 1.32472100 & 4.19358700 & -1.28594000 & $\mathrm{C}$ & -5.18173400 & 3.46885400 & -0.00687100 \\
\hline $\mathrm{H}$ & 1.32831600 & 4.71155300 & -2.25612200 & $\mathrm{C}$ & -4.51173700 & 4.47465400 & -0.70924100 \\
\hline $\mathrm{H}$ & 2.29719500 & 3.69594200 & -1.18746000 & $\mathrm{C}$ & -3.19052700 & 4.27558800 & -1.10830700 \\
\hline $\mathrm{C}$ & -0.27082300 & 5.86344900 & -0.22315000 & $\mathrm{C}$ & -2.53436600 & 3.08033800 & -0.80059400 \\
\hline $\mathrm{H}$ & -0.41407600 & 6.54682600 & 0.62414400 & $\mathrm{C}$ & -3.19396000 & 2.06767800 & -0.09308400 \\
\hline C & 1.11689900 & 5.20781500 & -0.14993800 & $\mathrm{H}$ & 2.43788100 & -0.64805300 & 2.47724200 \\
\hline $\mathrm{H}$ & 1.22220500 & 4.69518400 & 0.81780200 & $\mathrm{H}$ & 3.37460800 & 0.86443900 & 2.55942300 \\
\hline $\mathrm{H}$ & 1.90318800 & 5.97323700 & -0.18159700 & $\mathrm{H}$ & 1.22980300 & 1.00494500 & 3.86871700 \\
\hline $\mathrm{H}$ & -0.33432700 & 6.47579800 & -1.13515000 & $\mathrm{H}$ & 1.21896700 & 2.16686500 & 2.52539000 \\
\hline $\mathrm{O}$ & 0.44114000 & 2.28891700 & -2.44688100 & $\mathrm{H}$ & -3.38597400 & 0.81302700 & 2.55763600 \\
\hline \multirow[t]{2}{*}{$\mathrm{H}$} & 0.15296900 & 1.37423700 & -2.21148700 & $\mathrm{H}$ & -2.41164100 & -0.67994900 & 2.45685300 \\
\hline & & & & $\mathrm{H}$ & -1.24467300 & 2.15538600 & 2.52098700 \\
\hline \multirow{2}{*}{\multicolumn{4}{|c|}{$\begin{array}{l}\text { TS6 } \\
\text { imaginary frequency }=-421.44 \mathrm{~cm}^{-1}\end{array}$}} & $\mathrm{H}$ & -1.24733000 & 0.99248600 & 3.86431000 \\
\hline & & & & $\mathrm{H}$ & 2.77295600 & -0.64607200 & -2.30536100 \\
\hline $\mathrm{Ru}$ & -0.00045200 & 0.28071900 & 0.00673500 & $\mathrm{H}$ & 4.43641100 & -2.20038800 & -3.25550400 \\
\hline $\mathrm{P}$ & 2.32933400 & 0.48270300 & 0.32043500 & $\mathrm{H}$ & 6.17286000 & -3.22309600 & -1.79462200 \\
\hline $\mathrm{P}$ & -2.33322500 & 0.47288500 & 0.31473500 & $\mathrm{H}$ & 6.23081100 & -2.66256500 & 0.62778000 \\
\hline $\mathrm{N}$ & -0.00340400 & 0.48523100 & 2.21591800 & $\mathrm{H}$ & 4.59317300 & -1.09169400 & 1.57864600 \\
\hline $\mathrm{C}$ & 2.45330100 & 0.40753900 & 2.18302900 & $\mathrm{H}$ & 1.49089900 & 2.93559000 & -1.09167900 \\
\hline $\mathrm{C}$ & 1.22263300 & 1.09936600 & 2.77200000 & $\mathrm{H}$ & 2.65122800 & 5.05753700 & -1.66355000 \\
\hline C & -2.45663900 & 0.37827500 & 2.17489600 & $\mathrm{H}$ & 5.01262400 & 5.41087900 & -0.96596000 \\
\hline C & -1.23801200 & 1.08784800 & 2.76778700 & $\mathrm{H}$ & 6.21241500 & 3.61876200 & 0.27597200 \\
\hline C & 3.53723400 & -1.07173300 & -1.66110000 & $\mathrm{H}$ & 5.07385200 & 1.49844300 & 0.79879900 \\
\hline
\end{tabular}




\begin{tabular}{|c|c|c|c|c|c|c|c|}
\hline $\mathrm{H}$ & -2.72972800 & -0.68779100 & -2.30883200 & $\mathrm{H}$ & -2.77567800 & 0.00013400 & -0.38200300 \\
\hline $\mathrm{H}$ & -4.38280900 & -2.24674800 & -3.27000300 & $\mathrm{H}$ & -1.97592900 & -0.00008300 & 1.18738900 \\
\hline $\mathrm{H}$ & -6.15362800 & -3.23915400 & -1.82999300 & $\mathrm{C}$ & -1.01437500 & -1.26698200 & -0.28379600 \\
\hline $\mathrm{H}$ & -6.25592500 & -2.64531600 & 0.58305600 & $\mathrm{H}$ & -0.91450500 & -1.31122800 & -1.37731000 \\
\hline $\mathrm{H}$ & -4.62534100 & -1.07317700 & 1.54519100 & $\mathrm{H}$ & -1.56620500 & -2.16603300 & 0.01595600 \\
\hline $\mathrm{H}$ & -5.07398600 & 1.49138100 & 0.81512700 & $\mathrm{C}$ & 1.16008300 & -0.00002800 & 0.06869800 \\
\hline $\mathrm{H}$ & -6.21386300 & 3.61300300 & 0.30191600 & $\mathrm{C}$ & 0.39237800 & -1.28644600 & 0.35215200 \\
\hline $\mathrm{H}$ & -5.02086700 & 5.40516500 & -0.94644700 & $\mathrm{H}$ & 0.28767200 & -1.37792000 & 1.44455000 \\
\hline $\mathrm{H}$ & -2.66452200 & 5.05024200 & -1.66029100 & $\mathrm{H}$ & 0.99077500 & -2.13341000 & 0.00378300 \\
\hline $\mathrm{H}$ & -1.50240200 & 2.92688100 & -1.09803400 & $\mathrm{O}$ & 2.30096800 & -0.00004000 & -0.35352700 \\
\hline $\mathrm{C}$ & 0.00355600 & 0.06351600 & -1.83634300 & & & & \\
\hline $\mathrm{O}$ & 0.00662300 & -0.06983600 & -2.99632500 & b1- & & & \\
\hline $\mathrm{H}$ & -0.00121200 & 1.91687600 & -0.09116600 & $\mathrm{C}$ & 0.08966600 & 0.40730100 & 1.26397500 \\
\hline $\mathrm{C}$ & -1.30224200 & -3.42277200 & 0.90999400 & $\mathrm{H}$ & 1.12613400 & 0.78369800 & 1.29752300 \\
\hline $\mathrm{H}$ & -1.42988400 & -4.29315800 & 1.57509200 & $\mathrm{H}$ & -0.41276000 & 0.80208700 & 2.15947400 \\
\hline $\mathrm{H}$ & -2.18264300 & -2.79247900 & 1.05772500 & $\mathrm{C}$ & 0.08966600 & -1.13037300 & 1.27006200 \\
\hline $\mathrm{C}$ & -1.19639600 & -3.90659100 & -0.54162400 & $\mathrm{H}$ & 0.59110200 & -1.54130900 & 2.16394000 \\
\hline $\mathrm{H}$ & -2.07697300 & -4.51250700 & -0.79231800 & $\mathrm{H}$ & -0.95136700 & -1.49029600 & 1.31133800 \\
\hline $\mathrm{H}$ & -1.20992900 & -3.03831800 & -1.21160300 & $\mathrm{C}$ & -0.59835500 & 1.06356200 & 0.00000000 \\
\hline $\mathrm{C}$ & -0.05847500 & -2.70184000 & 1.45372900 & $\mathrm{H}$ & -1.64783800 & 0.56573700 & 0.00000000 \\
\hline $\mathrm{H}$ & -0.01474000 & -1.49600600 & 0.38893500 & $\mathrm{C}$ & 0.08966600 & 0.40730100 & -1.26397500 \\
\hline $\mathrm{C}$ & 1.25940800 & -3.38325200 & 1.04964900 & $\mathrm{H}$ & 1.12613400 & 0.78369800 & -1.29752300 \\
\hline $\mathrm{H}$ & 1.34303200 & -4.24841600 & 1.72834600 & $\mathrm{H}$ & -0.41276000 & 0.80208700 & -2.15947400 \\
\hline $\mathrm{H}$ & 2.09951800 & -2.72519100 & 1.28788400 & $\mathrm{C}$ & 0.74830100 & -1.69539400 & 0.00000000 \\
\hline $\mathrm{C}$ & 0.09313800 & -4.70498500 & -0.77840000 & $\mathrm{H}$ & 0.71003100 & -2.79754000 & 0.00000000 \\
\hline $\mathrm{H}$ & 0.15580500 & -5.02094300 & -1.82809800 & $\mathrm{C}$ & 0.08966600 & -1.13037300 & -1.27006200 \\
\hline $\mathrm{C}$ & 1.32537600 & -3.86988900 & -0.40353200 & $\mathrm{H}$ & -0.95136700 & -1.49029600 & -1.31133800 \\
\hline $\mathrm{H}$ & 1.38590400 & -3.00140600 & -1.06998900 & $\mathrm{H}$ & 0.59110200 & -1.54130900 & -2.16394000 \\
\hline $\mathrm{H}$ & 2.24548900 & -4.44906000 & -0.55606300 & $\mathrm{H}$ & 1.81591600 & -1.42128400 & 0.00000000 \\
\hline $\mathrm{H}$ & 0.07290000 & -5.62548100 & -0.17467400 & $\mathrm{O}$ & -0.57950000 & 2.37657300 & 0.00000000 \\
\hline $\mathrm{O}$ & -0.13171800 & -2.18243600 & 2.61139600 & & & & \\
\hline \multirow[t]{2}{*}{$\mathrm{H}$} & -0.00709500 & -0.52771700 & 2.48953700 & a1- & & & \\
\hline & & & & $\mathrm{C}$ & 1.98976800 & 1.15505800 & -0.15685300 \\
\hline b2 & & & & $\mathrm{C}$ & 0.60285800 & 1.20714200 & -0.32818900 \\
\hline $\mathrm{C}$ & -1.01431100 & 1.26701000 & -0.28370500 & $\mathrm{C}$ & -0.18956400 & 0.05853200 & -0.19426100 \\
\hline $\mathrm{H}$ & -0.91443600 & 1.31122200 & -1.37722300 & $\mathrm{C}$ & 0.44904200 & -1.15037200 & 0.10277600 \\
\hline $\mathrm{H}$ & -1.56621200 & 2.16604500 & 0.01597200 & $\mathrm{C}$ & 1.83279000 & -1.21416200 & 0.27909200 \\
\hline $\mathrm{C}$ & 0.39241400 & 1.28646300 & 0.35209200 & $\mathrm{C}$ & 2.61330400 & -0.05968000 & 0.15150800 \\
\hline $\mathrm{H}$ & 0.99088200 & 2.13330900 & 0.00353700 & $\mathrm{H}$ & 2.58882000 & 2.06019900 & -0.26567300 \\
\hline $\mathrm{H}$ & 0.28798700 & 1.37808000 & 1.44451500 & $\mathrm{H}$ & 0.12800300 & 2.15667200 & -0.57977100 \\
\hline $\mathrm{C}$ & -1.79153800 & 0.00001600 & 0.10273400 & $\mathrm{H}$ & -0.21981500 & -2.00803500 & 0.16559100 \\
\hline
\end{tabular}




\begin{tabular}{|c|c|c|c|c|c|c|c|}
\hline $\mathrm{H}$ & 2.31342000 & -2.16605200 & 0.51039700 & $\mathrm{C}$ & 2.45178100 & 5.93432800 & 0.93627400 \\
\hline $\mathrm{H}$ & 3.69435300 & -0.10470200 & 0.28201700 & $\mathrm{C}$ & 3.66201000 & 5.66520100 & 0.29124700 \\
\hline $\mathrm{C}$ & -1.74680800 & 0.00641100 & -0.38000300 & $\mathrm{C}$ & 3.85116500 & 4.43582600 & -0.34054000 \\
\hline $\mathrm{H}$ & -1.89589900 & 0.60144500 & -1.35110500 & $\mathrm{C}$ & 2.83978300 & 3.47180300 & -0.32164000 \\
\hline $\mathrm{O}$ & -2.24813900 & -1.21146000 & -0.36257600 & $\mathrm{C}$ & 1.62210500 & 3.72960000 & 0.32172200 \\
\hline $\mathrm{C}$ & -2.37799200 & 0.94622800 & 0.71842500 & $\mathrm{C}$ & -2.40841600 & 2.73743100 & -0.38710200 \\
\hline $\mathrm{H}$ & -2.18423700 & 0.51505900 & 1.71051100 & $\mathrm{C}$ & -3.49238600 & 3.31625700 & -1.04512100 \\
\hline $\mathrm{H}$ & -2.01476300 & 1.98855900 & 0.70856100 & $\mathrm{C}$ & -3.31462600 & 4.46235000 & -1.82424100 \\
\hline \multirow[t]{2}{*}{$\mathrm{H}$} & -3.46515700 & 0.95359400 & 0.56510500 & $\mathrm{C}$ & -2.04319400 & 5.02235900 & -1.94214100 \\
\hline & & & & $\mathrm{C}$ & -0.95620400 & 4.44913500 & -1.27687900 \\
\hline a1-CF3 & & & & $\mathrm{C}$ & -1.12646800 & 3.30550800 & -0.48245200 \\
\hline $\mathrm{C}$ & 2.66503400 & 1.18284500 & -0.35856300 & $\mathrm{C}$ & 4.42837100 & -1.50588700 & -0.25598400 \\
\hline $\mathrm{C}$ & 1.32986400 & 1.03558800 & -0.73549600 & $\mathrm{C}$ & 5.70170700 & -1.81177300 & -0.73367900 \\
\hline $\mathrm{C}$ & 0.63493400 & -0.13981200 & -0.43099700 & $\mathrm{C}$ & 5.93210000 & -3.01622300 & -1.40347100 \\
\hline $\mathrm{C}$ & 1.28717100 & -1.16809400 & 0.25773000 & $\mathrm{C}$ & 4.87965600 & -3.91162000 & -1.59049300 \\
\hline $\mathrm{C}$ & 2.62431500 & -1.02213600 & 0.62808900 & $\mathrm{C}$ & 3.60550500 & -3.61437900 & -1.09978300 \\
\hline $\mathrm{C}$ & 3.31550000 & 0.15254000 & 0.32326300 & $\mathrm{C}$ & 3.36597400 & -2.41158300 & -0.41917400 \\
\hline $\mathrm{H}$ & 3.19692900 & 2.09841300 & -0.60241700 & $\mathrm{C}$ & 0.93175600 & -4.67452800 & 0.65213400 \\
\hline $\mathrm{H}$ & 0.82605000 & 1.83803300 & -1.26872600 & $\mathrm{C}$ & 0.12547900 & -5.79236900 & 0.43264800 \\
\hline $\mathrm{H}$ & 0.74596700 & -2.07939500 & 0.48649400 & $\mathrm{C}$ & -0.93919900 & -5.72089500 & -0.46954500 \\
\hline $\mathrm{H}$ & 3.12679500 & -1.82764500 & 1.15686200 & $\mathrm{C}$ & -1.19453500 & -4.52732200 & -1.14696900 \\
\hline $\mathrm{H}$ & 4.35717400 & 0.26323100 & 0.61242200 & $\mathrm{C}$ & -0.39516700 & -3.40395200 & -0.91754800 \\
\hline $\mathrm{C}$ & -0.82324100 & -0.27589500 & -0.81344200 & $\mathrm{C}$ & 0.67945000 & -3.46475500 & -0.01796600 \\
\hline $\mathrm{H}$ & -1.02143700 & 0.31086800 & -1.72429700 & $\mathrm{H}$ & 0.01264600 & 3.30424800 & 2.66250900 \\
\hline $\mathrm{O}$ & -1.14580300 & -1.63951500 & -0.99781500 & $\mathrm{H}$ & -1.29512900 & 2.21581900 & 2.18220000 \\
\hline $\mathrm{H}$ & -2.10361900 & -1.69808600 & -1.14557000 & $\mathrm{H}$ & 1.57682000 & 1.34981500 & 2.86172500 \\
\hline $\mathrm{C}$ & -1.74674000 & 0.31958900 & 0.25903300 & $\mathrm{H}$ & 0.13251600 & 1.10204500 & 3.87216700 \\
\hline $\mathrm{F}$ & -3.04449200 & 0.08269300 & -0.08189400 & $\mathrm{H}$ & 2.18534500 & -2.87373900 & 2.51978400 \\
\hline $\mathrm{F}$ & -1.59148400 & 1.65423100 & 0.35195500 & $\mathrm{H}$ & 2.82706100 & -1.24303900 & 2.25686500 \\
\hline \multirow[t]{2}{*}{$\mathrm{F}$} & -1.55096400 & -0.21437400 & 1.47327800 & $\mathrm{H}$ & -0.10488700 & -2.03630700 & 2.67806200 \\
\hline & & & & $\mathrm{H}$ & 0.88537200 & -1.11589900 & 3.81210200 \\
\hline 13 & & & & $\mathrm{H}$ & 0.49361500 & 5.20961500 & 1.43014500 \\
\hline $\mathrm{Ru}$ & 0.84423200 & 0.20348400 & -0.08795000 & $\mathrm{H}$ & 2.29562300 & 6.89090900 & 1.42825800 \\
\hline $\mathrm{P}$ & 0.29354500 & 2.44178200 & 0.33481200 & $\mathrm{H}$ & 4.45156800 & 6.41215500 & 0.28164200 \\
\hline $\mathrm{P}$ & 1.70171500 & -1.94177500 & 0.23684000 & $\mathrm{H}$ & 4.78883200 & 4.22068700 & -0.84620400 \\
\hline $\mathrm{N}$ & 0.22271400 & -0.05500700 & 2.08318500 & $\mathrm{H}$ & 2.99418300 & 2.50557900 & -0.78966700 \\
\hline $\mathrm{C}$ & -0.21000900 & 2.36547400 & 2.14586800 & $\mathrm{H}$ & -2.56417100 & 1.82649500 & 0.18260100 \\
\hline $\mathrm{C}$ & 0.49447600 & 1.19334600 & 2.83554500 & $\mathrm{H}$ & -4.47362500 & 2.85639600 & -0.96244000 \\
\hline $\mathrm{C}$ & 1.96088300 & -1.89021900 & 2.09090900 & $\mathrm{H}$ & -4.15925300 & 4.90767600 & -2.34349800 \\
\hline $\mathrm{C}$ & 0.70722100 & -1.30358300 & 2.74448700 & $\mathrm{H}$ & -1.88978100 & 5.90694700 & -2.55495400 \\
\hline $\mathrm{C}$ & 1.43852800 & 4.97592300 & 0.94602700 & $\mathrm{H}$ & 0.02540800 & 4.89818900 & -1.38337900 \\
\hline
\end{tabular}




\begin{tabular}{|c|c|c|c|c|c|c|c|}
\hline $\mathrm{H}$ & 4.24233700 & -0.54988200 & 0.22493100 & $\mathrm{C}$ & -1.05989900 & -0.81976600 & 2.75002900 \\
\hline $\mathrm{H}$ & 6.51269700 & -1.10157300 & -0.59409400 & $\mathrm{C}$ & -0.83188400 & 2.58933500 & 2.00758000 \\
\hline $\mathrm{H}$ & 6.92293400 & -3.24934400 & -1.78444900 & $\mathrm{C}$ & 0.00124400 & 1.44655400 & 2.59227000 \\
\hline $\mathrm{H}$ & 5.04481800 & -4.84600500 & -2.12067600 & $\mathrm{C}$ & -3.96959900 & -3.55618700 & 1.09733400 \\
\hline $\mathrm{H}$ & 2.80004200 & -4.32398300 & -1.25440400 & $\mathrm{C}$ & -5.32705500 & -3.85386900 & 1.21399500 \\
\hline $\mathrm{H}$ & 1.76993100 & -4.75507300 & 1.33941900 & $\mathrm{C}$ & -6.28132500 & -2.99009700 & 0.66943200 \\
\hline $\mathrm{H}$ & 0.33082700 & -6.71916400 & 0.96217400 & $\mathrm{C}$ & -5.87006800 & -1.83125300 & 0.01063900 \\
\hline $\mathrm{H}$ & -1.56459300 & -6.59275300 & -0.64310300 & $\mathrm{C}$ & -4.51025200 & -1.52769900 & -0.09684900 \\
\hline $\mathrm{H}$ & -2.01690400 & -4.46605000 & -1.85518000 & $\mathrm{C}$ & -3.54414900 & -2.38517700 & 0.44562600 \\
\hline $\mathrm{H}$ & -0.60697600 & -2.46400600 & -1.41936700 & $\mathrm{C}$ & 0.31814600 & -3.66822400 & -0.60847700 \\
\hline $\mathrm{C}$ & 1.29912100 & 0.40211600 & -1.87243600 & $\mathrm{C}$ & 0.86547800 & -4.72671400 & -1.33219400 \\
\hline $\mathrm{O}$ & 1.57817400 & 0.52367800 & -2.99648400 & $\mathrm{C}$ & 0.04206100 & -5.57040500 & -2.08160700 \\
\hline $\mathrm{H}$ & -0.75029500 & -0.25339300 & -0.55990600 & $\mathrm{C}$ & -1.33369600 & -5.34429900 & -2.10413200 \\
\hline $\mathrm{H}$ & 2.36934600 & 0.63743100 & 0.48440000 & $\mathrm{C}$ & -1.88650600 & -4.29042600 & -1.37224500 \\
\hline $\mathrm{H}$ & -0.79554600 & -0.14899800 & 2.00835400 & $\mathrm{C}$ & -1.06937300 & -3.44507800 & -0.60631500 \\
\hline $\mathrm{C}$ & -6.09235800 & -2.14939900 & -1.47414400 & $\mathrm{C}$ & -3.33203700 & 3.50946600 & -0.18156400 \\
\hline $\mathrm{C}$ & -4.98418200 & -2.32618300 & -0.64170600 & $\mathrm{C}$ & -4.30754000 & 4.42549200 & -0.57220800 \\
\hline $\mathrm{C}$ & -4.59423400 & -1.32552300 & 0.25450700 & $\mathrm{C}$ & -3.94316800 & 5.60976000 & -1.21787200 \\
\hline $\mathrm{C}$ & -5.33421500 & -0.13562700 & 0.29994000 & $\mathrm{C}$ & -2.59640000 & 5.87112200 & -1.46787800 \\
\hline $\mathrm{C}$ & -6.44024800 & 0.04500600 & -0.53022700 & $\mathrm{C}$ & -1.61519400 & 4.96164600 & -1.06492000 \\
\hline $\mathrm{C}$ & -6.82367700 & -0.96253500 & -1.42033700 & $\mathrm{C}$ & -1.97051200 & 3.77313200 & -0.41019300 \\
\hline $\mathrm{H}$ & -6.37860400 & -2.93558500 & -2.16811200 & $\mathrm{C}$ & 1.39188100 & 4.43284800 & 0.42837100 \\
\hline $\mathrm{H}$ & -4.41226800 & -3.25075900 & -0.69150800 & $\mathrm{C}$ & 2.63019100 & 4.98374600 & 0.09422500 \\
\hline $\mathrm{H}$ & -5.03116300 & 0.65113900 & 0.98522300 & $\mathrm{C}$ & 3.39036400 & 4.42435100 & -0.93578000 \\
\hline $\mathrm{H}$ & -7.00631700 & 0.97215100 & -0.48290100 & $\mathrm{C}$ & 2.90827800 & 3.31083400 & -1.62671800 \\
\hline $\mathrm{H}$ & -7.68378800 & -0.82020300 & -2.06921900 & $\mathrm{C}$ & 1.67529500 & 2.75017100 & -1.28227700 \\
\hline $\mathrm{C}$ & -3.41311100 & -1.54089900 & 1.19052900 & $\mathrm{C}$ & 0.90034700 & 3.30612900 & -0.25285300 \\
\hline $\mathrm{H}$ & -2.83686600 & -2.40410800 & 0.82761000 & $\mathrm{H}$ & -1.72947900 & -2.88144400 & 2.61900500 \\
\hline $\mathrm{O}$ & -2.55983100 & -0.39135800 & 1.24090700 & $\mathrm{H}$ & -0.10074600 & -2.60286700 & 1.99176000 \\
\hline $\mathrm{H}$ & -1.99787100 & -0.36311500 & 0.41956600 & $\mathrm{H}$ & -2.06483400 & -0.40385400 & 2.86718900 \\
\hline $\mathrm{C}$ & -3.86903500 & -1.82348700 & 2.62373800 & $\mathrm{H}$ & -0.61332100 & -0.93405700 & 3.75011600 \\
\hline $\mathrm{H}$ & -4.41938500 & -0.96508900 & 3.02428100 & $\mathrm{H}$ & -0.49864300 & 3.54823600 & 2.42105800 \\
\hline $\mathrm{H}$ & -4.52679300 & -2.69820900 & 2.65539000 & $\mathrm{H}$ & -1.89154200 & 2.46819400 & 2.25114400 \\
\hline \multirow[t]{2}{*}{$\mathrm{H}$} & -3.00341100 & -2.01146000 & 3.26902900 & $\mathrm{H}$ & 1.06352000 & 1.66686100 & 2.45740100 \\
\hline & & & & $\mathrm{H}$ & -0.17315800 & 1.36248600 & 3.67325200 \\
\hline 13-CF3 & & & & $\mathrm{H}$ & -3.23861500 & -4.25090400 & 1.50324900 \\
\hline $\mathrm{Ru}$ & -1.10397600 & 0.23517000 & -0.17181100 & $\mathrm{H}$ & -5.63934400 & -4.76047700 & 1.72576900 \\
\hline $\mathrm{P}$ & -1.74648900 & -1.97433700 & 0.29281200 & $\mathrm{H}$ & -7.33940400 & -3.22235100 & 0.75819700 \\
\hline $\mathrm{P}$ & -0.72801900 & 2.51911000 & 0.14040900 & $\mathrm{H}$ & -6.60648600 & -1.15669500 & -0.41810200 \\
\hline $\mathrm{N}$ & -0.25886700 & 0.12745700 & 1.93556400 & $\mathrm{H}$ & -4.18971100 & -0.61497300 & -0.58730100 \\
\hline $\mathrm{C}$ & -1.11048300 & -2.18095800 & 2.04989600 & $\mathrm{H}$ & 0.98412000 & -3.00467400 & -0.06577600 \\
\hline
\end{tabular}




\begin{tabular}{|c|c|c|c|c|c|c|c|}
\hline $\mathrm{H}$ & 1.94127300 & -4.88127200 & -1.32100400 & $\mathrm{Ru}$ & 0.70348200 & 0.50517200 & -0.15057200 \\
\hline $\mathrm{H}$ & 0.47160400 & -6.39068200 & -2.65066500 & $\mathrm{P}$ & -0.96922600 & 2.14154900 & 0.27540100 \\
\hline $\mathrm{H}$ & -1.98399400 & -5.98642000 & -2.69254700 & $\mathrm{P}$ & 2.49774100 & -0.97644600 & 0.24819800 \\
\hline $\mathrm{H}$ & -2.95905700 & -4.13113600 & -1.40263700 & $\mathrm{~N}$ & 0.05434500 & -0.20745900 & 1.83219100 \\
\hline $\mathrm{H}$ & -3.62044100 & 2.57148100 & 0.28402300 & $\mathrm{C}$ & -1.52092700 & 1.66291000 & 1.99516100 \\
\hline $\mathrm{H}$ & -5.35552800 & 4.20743600 & -0.38334500 & $\mathrm{C}$ & -0.38451600 & 0.90733000 & 2.69490700 \\
\hline $\mathrm{H}$ & -4.70515200 & 6.31859300 & -1.53110500 & $\mathrm{C}$ & 2.43076900 & -1.00689300 & 2.10782200 \\
\hline $\mathrm{H}$ & -2.30275100 & 6.78420400 & -1.97917700 & $\mathrm{C}$ & 0.95811500 & -1.15991000 & 2.51249100 \\
\hline $\mathrm{H}$ & -0.57301700 & 5.18305400 & -1.26758600 & $\mathrm{C}$ & -1.27686800 & 4.83989600 & 1.07432000 \\
\hline $\mathrm{H}$ & 0.80484100 & 4.89560800 & 1.21710100 & $\mathrm{C}$ & -0.88842200 & 6.17369600 & 1.19376000 \\
\hline $\mathrm{H}$ & 2.99924800 & 5.85082600 & 0.63570800 & $\mathrm{C}$ & 0.33270000 & 6.59737400 & 0.66245200 \\
\hline $\mathrm{H}$ & 4.35277000 & 4.85591900 & -1.19780900 & $\mathrm{C}$ & 1.15843500 & 5.68166300 & 0.01043200 \\
\hline $\mathrm{H}$ & 3.49056600 & 2.87430400 & -2.43415200 & $\mathrm{C}$ & 0.77156900 & 4.34388300 & -0.10423500 \\
\hline $\mathrm{H}$ & 1.31006600 & 1.86595500 & -1.79674400 & $\mathrm{C}$ & -0.44824600 & 3.90516000 & 0.42837600 \\
\hline $\mathrm{C}$ & -1.75699100 & 0.31045300 & -1.90442200 & $\mathrm{C}$ & -3.31843700 & 1.06867000 & -0.80309500 \\
\hline $\mathrm{O}$ & -2.16066000 & 0.35715900 & -2.99522900 & $\mathrm{C}$ & -4.45625400 & 1.06347100 & -1.61044300 \\
\hline $\mathrm{H}$ & 0.45851900 & -0.16876000 & -0.79966200 & $\mathrm{C}$ & -4.78754500 & 2.18501600 & -2.37514800 \\
\hline $\mathrm{H}$ & -2.57753600 & 0.63234600 & 0.52717100 & $\mathrm{C}$ & -3.97272100 & 3.31681000 & -2.33201300 \\
\hline $\mathrm{H}$ & 0.65673000 & -0.30693100 & 1.78886400 & $\mathrm{C}$ & -2.83534000 & 3.33363500 & -1.52129600 \\
\hline $\mathrm{C}$ & 6.11805100 & -0.92980500 & -2.04957600 & $\mathrm{C}$ & -2.50415200 & 2.21404000 & -0.74305200 \\
\hline $\mathrm{C}$ & 5.19924400 & -0.30817000 & -1.20190500 & $\mathrm{C}$ & 4.58664700 & 0.85135300 & -0.18042400 \\
\hline $\mathrm{C}$ & 4.38186800 & -1.06996000 & -0.36112600 & $\mathrm{C}$ & 5.90219500 & 1.24033900 & -0.43404700 \\
\hline $\mathrm{C}$ & 4.49760400 & -2.46522100 & -0.37241300 & $\mathrm{C}$ & 6.88546000 & 0.27801000 & -0.67209800 \\
\hline $\mathrm{C}$ & 5.41471200 & -3.08696300 & -1.21912700 & $\mathrm{C}$ & 6.54709400 & -1.07597600 & -0.65240000 \\
\hline C & 6.22689900 & -2.32117700 & -2.05985800 & $\mathrm{C}$ & 5.23305500 & -1.46860900 & -0.39231500 \\
\hline $\mathrm{H}$ & 6.74378500 & -0.32827900 & -2.70353300 & $\mathrm{C}$ & 4.23814100 & -0.50868200 & -0.14894600 \\
\hline $\mathrm{H}$ & 5.11273300 & 0.77601600 & -1.19618500 & $\mathrm{C}$ & 2.67721900 & -3.82731300 & 0.51768200 \\
\hline $\mathrm{H}$ & 3.86359200 & -3.05468600 & 0.28178300 & $\mathrm{C}$ & 2.55096900 & -5.13303500 & 0.03766800 \\
\hline $\mathrm{H}$ & 5.49814200 & -4.17067900 & -1.22228500 & $\mathrm{C}$ & 2.12036700 & -5.36097200 & -1.27001800 \\
\hline $\mathrm{H}$ & 6.93869000 & -2.80752800 & -2.72155400 & $\mathrm{C}$ & 1.81185300 & -4.27826700 & -2.09675700 \\
\hline $\mathrm{C}$ & 3.39576600 & -0.38844100 & 0.57330900 & $\mathrm{C}$ & 1.92693500 & -2.97497700 & -1.61409700 \\
\hline $\mathrm{H}$ & 3.22018700 & 0.63994100 & 0.22927000 & $\mathrm{C}$ & 2.36585100 & -2.73111100 & -0.30213300 \\
\hline $\mathrm{O}$ & 2.17465700 & -1.08956700 & 0.70721100 & $\mathrm{H}$ & -1.83592000 & 2.53768000 & 2.57220200 \\
\hline $\mathrm{H}$ & 1.52885300 & -0.75640500 & 0.00819800 & $\mathrm{H}$ & -2.37069000 & 0.98327400 & 1.88271500 \\
\hline $\mathrm{C}$ & 3.97110400 & -0.25511900 & 1.98595500 & $\mathrm{H}$ & 0.44830000 & 1.58600800 & 2.92834400 \\
\hline $\mathrm{F}$ & 3.11502500 & 0.41555900 & 2.79984100 & $\mathrm{H}$ & -0.76617400 & 0.51254200 & 3.65037800 \\
\hline $\mathrm{F}$ & 5.12827800 & 0.43606900 & 1.97153100 & $\mathrm{H}$ & 3.06386800 & -1.78354900 & 2.55344700 \\
\hline \multirow[t]{2}{*}{$\mathrm{F}$} & 4.20578300 & -1.44925200 & 2.55979800 & $\mathrm{H}$ & 2.82257600 & -0.03538800 & 2.42879400 \\
\hline & & & & $\mathrm{H}$ & 0.63060000 & -2.17736500 & 2.25850600 \\
\hline \multicolumn{4}{|c|}{ TS7 } & $\mathrm{H}$ & 0.86569900 & -1.06521800 & 3.60381100 \\
\hline \multicolumn{4}{|c|}{ imaginary frequency $=-894.40 \mathrm{~cm}^{-1}$} & $\mathrm{H}$ & -2.23816800 & 4.52980400 & 1.47505700 \\
\hline
\end{tabular}




\begin{tabular}{|c|c|c|c|c|c|c|c|}
\hline $\mathrm{H}$ & -1.53954000 & 6.88207200 & 1.69901900 & $\mathrm{H}$ & -2.64434000 & -4.11528900 & 2.80739200 \\
\hline $\mathrm{H}$ & 0.63571200 & 7.63690200 & 0.75525700 & $\mathrm{H}$ & -1.49015300 & -2.82633200 & 3.21379000 \\
\hline $\mathrm{H}$ & 2.10724800 & 6.00452400 & -0.40964300 & & & & \\
\hline $\mathrm{H}$ & 1.42090400 & 3.63533500 & -0.60593300 & \multirow{2}{*}{\multicolumn{4}{|c|}{$\begin{array}{l}\text { TS7-CF3 } \\
\text { imaginary frequency }=-604.55 \mathrm{~cm}^{-1}\end{array}$}} \\
\hline $\mathrm{H}$ & -3.05915800 & 0.17876300 & -0.22990100 & & & & \\
\hline $\mathrm{H}$ & -5.07758300 & 0.17234700 & -1.64131000 & $\mathrm{Ru}$ & 1.10140100 & 0.36463100 & -0.29518000 \\
\hline $\mathrm{H}$ & -5.67261000 & 2.17438700 & -3.00619700 & $\mathrm{P}$ & -0.11281800 & 2.37049300 & 0.10693900 \\
\hline $\mathrm{H}$ & -4.21831800 & 4.19131800 & -2.92912400 & $\mathrm{P}$ & 2.37017200 & -1.56785100 & 0.17956200 \\
\hline $\mathrm{H}$ & -2.21223100 & 4.22177800 & -1.49796800 & $\mathrm{~N}$ & -0.01802600 & -0.27495200 & 1.48520600 \\
\hline $\mathrm{H}$ & 3.82135700 & 1.60248700 & -0.01126200 & $\mathrm{C}$ & -0.98692200 & 1.96503800 & 1.70800800 \\
\hline $\mathrm{H}$ & 6.15582600 & 2.29686100 & -0.45534900 & $\mathrm{C}$ & -0.21787200 & 0.84564500 & 2.42317900 \\
\hline $\mathrm{H}$ & 7.90835500 & 0.58186600 & -0.87781400 & $\mathrm{C}$ & 1.97418400 & -1.73904900 & 1.98675000 \\
\hline $\mathrm{H}$ & 7.30540200 & -1.83093600 & -0.84251100 & $\mathrm{C}$ & 0.46398200 & -1.50542200 & 2.14333600 \\
\hline $\mathrm{H}$ & 4.98400900 & -2.52489700 & -0.38503400 & $\mathrm{C}$ & 0.21279400 & 4.99427000 & 1.11541900 \\
\hline $\mathrm{H}$ & 3.02065500 & -3.67318200 & 1.53603300 & $\mathrm{C}$ & 0.92472700 & 6.15770500 & 1.40452200 \\
\hline $\mathrm{H}$ & 2.788666200 & -5.97052200 & 0.68811900 & $\mathrm{C}$ & 2.27456800 & 6.25703000 & 1.05669300 \\
\hline $\mathrm{H}$ & 2.02092800 & -6.37710800 & -1.64178500 & $\mathrm{C}$ & 2.90422800 & 5.18952700 & 0.41698300 \\
\hline $\mathrm{H}$ & 1.47153500 & -4.44759600 & -3.11458000 & $\mathrm{C}$ & 2.19189900 & 4.02161700 & 0.13195300 \\
\hline $\mathrm{H}$ & 1.66672800 & -2.13863300 & -2.25734300 & $\mathrm{C}$ & 0.83923600 & 3.90726900 & 0.47983900 \\
\hline $\mathrm{C}$ & 1.25627400 & 1.16262900 & -1.82171300 & $\mathrm{C}$ & -2.50623100 & 2.07991900 & -1.32272400 \\
\hline $\mathrm{O}$ & 1.62617600 & 1.59324800 & -2.83517700 & $\mathrm{C}$ & -3.47896400 & 2.44279900 & -2.25440100 \\
\hline $\mathrm{H}$ & -0.34660300 & -0.83772100 & -1.14609900 & $\mathrm{C}$ & -3.37946700 & 3.65153400 & -2.94824600 \\
\hline $\mathrm{H}$ & 1.67731400 & 1.55193400 & 0.54973600 & $\mathrm{C}$ & -2.29714000 & 4.49801800 & -2.70747200 \\
\hline $\mathrm{H}$ & -0.99152700 & -0.82255400 & 1.52505200 & $\mathrm{C}$ & -1.32167900 & 4.14429100 & -1.77207600 \\
\hline $\mathrm{C}$ & -4.93036600 & -4.54945300 & -0.87922700 & $\mathrm{C}$ & -1.42159400 & 2.93712300 & -1.06415400 \\
\hline $\mathrm{C}$ & -3.73371300 & -4.18934000 & -0.25329200 & $\mathrm{C}$ & 4.89604800 & -0.33526000 & 0.22926200 \\
\hline $\mathrm{C}$ & -3.63481800 & -3.00928700 & 0.49230600 & $\mathrm{C}$ & 6.29090100 & -0.29929300 & 0.21963800 \\
\hline $\mathrm{C}$ & -4.76899500 & -2.19141000 & 0.59613400 & $\mathrm{C}$ & 7.02254000 & -1.47835000 & 0.06610000 \\
\hline $\mathrm{C}$ & -5.96705600 & -2.54586200 & -0.02537600 & $\mathrm{C}$ & 6.35269500 & -2.69500800 & -0.07499400 \\
\hline $\mathrm{C}$ & -6.05295600 & -3.72818700 & -0.76712200 & $\mathrm{C}$ & 4.95772200 & -2.73476500 & -0.06034400 \\
\hline $\mathrm{H}$ & -4.98284600 & -5.46825400 & -1.45882200 & $\mathrm{C}$ & 4.21320000 & -1.55470100 & 0.09583100 \\
\hline $\mathrm{H}$ & -2.86036600 & -4.83210600 & -0.35014400 & $\mathrm{C}$ & 1.69760500 & -4.35726600 & 0.09947600 \\
\hline $\mathrm{H}$ & -4.69490200 & -1.26785000 & 1.16256800 & $\mathrm{C}$ & 1.33767000 & -5.53196800 & -0.56607200 \\
\hline $\mathrm{H}$ & -6.83755900 & -1.90044600 & 0.07050000 & $\mathrm{C}$ & 1.18703400 & -5.53475900 & -1.95307200 \\
\hline $\mathrm{H}$ & -6.98499400 & -4.00348400 & -1.25443500 & $\mathrm{C}$ & 1.39367600 & -4.35637300 & -2.67454800 \\
\hline $\mathrm{C}$ & -2.33960300 & -2.62774300 & 1.20949600 & $\mathrm{C}$ & 1.74187700 & -3.18149400 & -2.00943900 \\
\hline $\mathrm{H}$ & -1.53279000 & -3.23750800 & 0.75169600 & $\mathrm{C}$ & 1.90374600 & -3.16633600 & -0.61368000 \\
\hline $\mathrm{O}$ & -2.08280100 & -1.26185300 & 1.07566400 & $\mathrm{H}$ & -1.08503100 & 2.85275000 & 2.34014000 \\
\hline $\mathrm{H}$ & -0.84064400 & -0.85249700 & -0.53828500 & $\mathrm{H}$ & -1.98680500 & 1.60047700 & 1.45679000 \\
\hline $\mathrm{C}$ & -2.43203900 & -3.04455300 & 2.69506000 & $\mathrm{H}$ & 0.73586300 & 1.22977100 & 2.81655400 \\
\hline $\mathrm{H}$ & -3.23096100 & -2.48034100 & 3.19011400 & $\mathrm{H}$ & -0.81874100 & 0.51000600 & 3.28140400 \\
\hline
\end{tabular}




\begin{tabular}{|c|c|c|c|c|c|c|c|}
\hline $\mathrm{H}$ & 2.29705900 & -2.69634600 & 2.41398500 & $\mathrm{H}$ & -2.61813500 & -2.65449200 & 0.93967400 \\
\hline $\mathrm{H}$ & 2.54250900 & -0.94588500 & 2.48534100 & $\mathrm{O}$ & -2.28757600 & -0.65300400 & 0.46092700 \\
\hline $\mathrm{H}$ & -0.06505000 & -2.36132500 & 1.70000800 & $\mathrm{H}$ & -0.58784400 & -0.44324800 & -1.11532100 \\
\hline $\mathrm{H}$ & 0.20134000 & -1.50067000 & 3.20958000 & $\mathrm{C}$ & -3.55199900 & -1.41570400 & 2.35690600 \\
\hline $\mathrm{H}$ & -0.84071300 & 4.93915700 & 1.37604600 & $\mathrm{~F}$ & -4.41114900 & -2.35706400 & 2.80544700 \\
\hline $\mathrm{H}$ & 0.42562800 & 6.98650000 & 1.89950500 & $\mathrm{~F}$ & -4.13758000 & -0.21110900 & 2.52540900 \\
\hline $\mathrm{H}$ & 2.82994100 & 7.16351000 & 1.28226300 & $\mathrm{~F}$ & -2.47452100 & -1.44283100 & 3.19039900 \\
\hline $\mathrm{H}$ & 3.95239400 & 5.26067700 & 0.13913000 & & & & \\
\hline $\mathrm{H}$ & 2.68978000 & 3.19379900 & -0.35976600 & 14 & & & \\
\hline $\mathrm{H}$ & -2.58874300 & 1.12592300 & -0.80552300 & $\mathrm{Ru}$ & 0.75988800 & 0.41402100 & -0.15920800 \\
\hline $\mathrm{H}$ & -4.31188400 & 1.76981200 & -2.44065400 & $\mathrm{P}$ & -0.73323600 & 2.20748100 & 0.28803100 \\
\hline $\mathrm{H}$ & -4.13741700 & 3.92828800 & -3.67649500 & $\mathrm{P}$ & 2.44142200 & -1.18872100 & 0.27642500 \\
\hline $\mathrm{H}$ & -2.20649600 & 5.43725000 & -3.24702800 & $\mathrm{~N}$ & 0.01495500 & -0.24642600 & 1.77877100 \\
\hline $\mathrm{H}$ & -0.48609700 & 4.81406300 & -1.59701300 & $\mathrm{C}$ & -1.29115600 & 1.81052400 & 2.02609700 \\
\hline $\mathrm{H}$ & 4.32983900 & 0.58456700 & 0.33666900 & $\mathrm{C}$ & -0.25266300 & 0.88313800 & 2.67378400 \\
\hline $\mathrm{H}$ & 6.80488500 & 0.65259700 & 0.32318300 & $\mathrm{C}$ & 2.28380200 & -1.29568800 & 2.12310700 \\
\hline $\mathrm{H}$ & 8.10876000 & -1.44878500 & 0.05105100 & $\mathrm{C}$ & 0.77311500 & -1.32103500 & 2.42763000 \\
\hline $\mathrm{H}$ & 6.91519100 & -3.61651400 & -0.19976700 & $\mathrm{C}$ & -0.77567400 & 4.93756900 & 1.02876000 \\
\hline $\mathrm{H}$ & 4.44998500 & -3.68709600 & -0.17640600 & $\mathrm{C}$ & -0.26110600 & 6.22997000 & 1.12197800 \\
\hline $\mathrm{H}$ & 1.81462600 & -4.38007600 & 1.17841000 & $\mathrm{C}$ & 0.99346600 & 6.52457700 & 0.58116800 \\
\hline $\mathrm{H}$ & 1.17502700 & -6.44359100 & 0.00271600 & $\mathrm{C}$ & 1.72567700 & 5.52169000 & -0.05385500 \\
\hline $\mathrm{H}$ & 0.90602400 & -6.44856600 & -2.46941200 & $\mathrm{C}$ & 1.21211200 & 4.22501100 & -0.14236100 \\
\hline $\mathrm{H}$ & 1.27511600 & -4.34924600 & -3.75457800 & $\mathrm{C}$ & -0.04219000 & 3.91556000 & 0.40008900 \\
\hline $\mathrm{H}$ & 1.88481000 & -2.26562900 & -2.57728200 & $\mathrm{C}$ & -3.23958400 & 1.40911500 & -0.69294600 \\
\hline $\mathrm{C}$ & 2.07550800 & 0.97161500 & -1.78749600 & $\mathrm{C}$ & -4.38293200 & 1.51365300 & -1.48552300 \\
\hline $\mathrm{O}$ & 2.71010600 & 1.36108100 & -2.67967300 & $\mathrm{C}$ & -4.57189400 & 2.62518200 & -2.31069800 \\
\hline $\mathrm{H}$ & -0.00932500 & -0.63493900 & -1.59504200 & $\mathrm{C}$ & -3.60855800 & 3.63392900 & -2.34113900 \\
\hline $\mathrm{H}$ & 2.17677800 & 1.04986600 & 0.65520800 & $\mathrm{C}$ & -2.46500900 & 3.53941000 & -1.54434900 \\
\hline $\mathrm{H}$ & -1.26497000 & -0.55280000 & 0.99175500 & $\mathrm{C}$ & -2.27341500 & 2.43070900 & -0.70609400 \\
\hline $\mathrm{C}$ & -5.83927100 & -3.18302700 & -1.33325100 & $\mathrm{C}$ & 4.65167000 & 0.51018000 & -0.10392000 \\
\hline $\mathrm{C}$ & -4.72525400 & -3.05645600 & -0.49949900 & $\mathrm{C}$ & 6.00115800 & 0.81337400 & -0.28983600 \\
\hline $\mathrm{C}$ & -4.34582200 & -1.80880000 & 0.00369800 & $\mathrm{C}$ & 6.93928200 & -0.21168400 & -0.42313300 \\
\hline $\mathrm{C}$ & -5.10631300 & -0.68185800 & -0.33815800 & $\mathrm{C}$ & 6.52219300 & -1.54293700 & -0.36726700 \\
\hline $\mathrm{C}$ & -6.22075800 & -0.80533100 & -1.16713900 & $\mathrm{C}$ & 5.17409100 & -1.84899900 & -0.17709100 \\
\hline $\mathrm{C}$ & -6.59051900 & -2.05670300 & -1.66913700 & $\mathrm{C}$ & 4.22316300 & -0.82494700 & -0.03929300 \\
\hline $\mathrm{H}$ & -6.11681600 & -4.16020800 & -1.72027300 & $\mathrm{C}$ & 2.32980200 & -4.05606100 & 0.42344600 \\
\hline $\mathrm{H}$ & -4.14119200 & -3.93734300 & -0.24204300 & $\mathrm{C}$ & 2.14756100 & -5.32428600 & -0.13472400 \\
\hline $\mathrm{H}$ & -4.81901600 & 0.28894000 & 0.05093500 & $\mathrm{C}$ & 1.87230400 & -5.45947300 & -1.49567200 \\
\hline $\mathrm{H}$ & -6.80611700 & 0.07577100 & -1.41885800 & $\mathrm{C}$ & 1.77455900 & -4.32021300 & -2.29871600 \\
\hline $\mathrm{H}$ & -7.45782300 & -2.15108900 & -2.31750800 & $\mathrm{C}$ & 1.94441100 & -3.05423300 & -1.73974700 \\
\hline $\mathrm{C}$ & -3.12171900 & -1.67051000 & 0.90085100 & $\mathrm{C}$ & 2.22974700 & -2.90419300 & -0.37162400 \\
\hline
\end{tabular}




\begin{tabular}{|c|c|c|c|c|c|c|c|}
\hline $\mathrm{H}$ & -1.43601100 & 2.72650100 & 2.60765200 & $\mathrm{H}$ & -5.01191700 & -0.96200100 & 1.42593900 \\
\hline $\mathrm{H}$ & -2.25002900 & 1.28922300 & 1.95578600 & $\mathrm{H}$ & -7.26202900 & -1.32125200 & 0.42831600 \\
\hline $\mathrm{H}$ & 0.65240800 & 1.46533500 & 2.92691700 & $\mathrm{H}$ & -7.64300500 & -3.22456000 & -1.12944700 \\
\hline $\mathrm{H}$ & -0.67157600 & 0.52092900 & 3.62867900 & $\mathrm{C}$ & -2.76094300 & -2.46571500 & 1.10648400 \\
\hline $\mathrm{H}$ & 2.82464400 & -2.14517100 & 2.55895500 & $\mathrm{H}$ & -1.99722200 & -2.84482500 & 0.40543800 \\
\hline $\mathrm{H}$ & 2.73894300 & -0.37665400 & 2.50903900 & $\mathrm{O}$ & -2.55596900 & -1.08860900 & 1.35791600 \\
\hline $\mathrm{H}$ & 0.38310400 & -2.29406400 & 2.08899500 & $\mathrm{H}$ & -0.82870900 & -0.50582400 & -0.88595000 \\
\hline $\mathrm{H}$ & 0.62740100 & -1.29720300 & 3.51902800 & $\mathrm{C}$ & -2.66280000 & -3.28319200 & 2.40831900 \\
\hline $\mathrm{H}$ & -1.76028100 & 4.72755600 & 1.43790300 & $\mathrm{H}$ & -3.41649000 & -2.93376500 & 3.12234700 \\
\hline $\mathrm{H}$ & -0.83974800 & 7.00688700 & 1.61459800 & $\mathrm{H}$ & -2.82818000 & -4.35141200 & 2.22414000 \\
\hline $\mathrm{H}$ & 1.39492900 & 7.53189500 & 0.65359100 & $\mathrm{H}$ & -1.67337400 & -3.16074100 & 2.86406500 \\
\hline $\mathrm{H}$ & 2.69984200 & 5.74398000 & -0.48115300 & & & & \\
\hline $\mathrm{H}$ & 1.78861600 & 3.44845100 & -0.63205600 & $\mathrm{H}_{2}$ & & & \\
\hline $\mathrm{H}$ & -3.10199500 & 0.52569700 & -0.07211300 & $\mathrm{H}$ & 0.00000000 & 0.00000000 & 0.3713940 \\
\hline $\mathrm{H}$ & -5.12185300 & 0.71715100 & -1.45633900 & $\mathrm{H}$ & 0.00000000 & 0.00000000 & -0.37139400 \\
\hline $\mathrm{H}$ & -5.46177300 & 2.70153100 & -2.93020900 & & & & \\
\hline $\mathrm{H}$ & -3.74284200 & 4.49945300 & -2.98491600 & 15 & & & \\
\hline $\mathrm{H}$ & -1.72662000 & 4.33395600 & -1.57809700 & $\mathrm{Ru}$ & 0.31127800 & -0.51927400 & -0.12142900 \\
\hline $\mathrm{H}$ & 3.92282200 & 1.30903900 & -0.01153000 & $\mathrm{P}$ & 2.48693500 & 0.22398000 & 0.33601300 \\
\hline $\mathrm{H}$ & 6.31686200 & 1.85210300 & -0.33906600 & $\mathrm{P}$ & -1.71011800 & -1.62996400 & 0.25293000 \\
\hline $\mathrm{H}$ & 7.98899900 & 0.02507900 & -0.57508700 & $\mathrm{~N}$ & -0.06865300 & 0.26025000 & 1.97523000 \\
\hline $\mathrm{H}$ & 7.24581400 & -2.34667600 & -0.47499500 & $\mathrm{C}$ & 2.32121600 & 0.81002300 & 2.11720400 \\
\hline $\mathrm{H}$ & 4.86315500 & -2.88848400 & -0.14229700 & $\mathrm{C}$ & 1.15275500 & 0.09060300 & 2.79890600 \\
\hline $\mathrm{H}$ & 2.54792100 & -3.97525100 & 1.48365600 & $\mathrm{C}$ & -1.74489200 & -1.61753900 & 2.12252700 \\
\hline $\mathrm{H}$ & 2.22104600 & -6.20515500 & 0.49756000 & $\mathrm{C}$ & -1.32961500 & -0.22562700 & 2.60320200 \\
\hline $\mathrm{H}$ & 1.73079800 & -6.44612300 & -1.92835100 & $\mathrm{C}$ & 5.09080900 & -0.67888200 & 1.03050400 \\
\hline $\mathrm{H}$ & 1.55813100 & -4.41631900 & -3.35921300 & $\mathrm{C}$ & 6.11766700 & -1.61781600 & 1.12129000 \\
\hline $\mathrm{H}$ & 1.85028100 & -2.17240900 & -2.36849200 & $\mathrm{C}$ & 5.93414600 & -2.90572900 & 0.60995300 \\
\hline $\mathrm{C}$ & 1.39848400 & 1.02415000 & -1.82566300 & $\mathrm{C}$ & 4.72115100 & -3.24614300 & 0.01138100 \\
\hline $\mathrm{O}$ & 1.83227600 & 1.42065900 & -2.82942100 & $\mathrm{C}$ & 3.68827400 & -2.30801600 & -0.07065300 \\
\hline $\mathrm{H}$ & -0.27136100 & -1.04879000 & -0.97067600 & $\mathrm{C}$ & 3.86036900 & -1.01428400 & 0.43778800 \\
\hline $\mathrm{H}$ & 1.79749600 & 1.36835300 & 0.58236000 & $\mathrm{C}$ & 2.75126000 & 2.93299500 & -0.42089000 \\
\hline $\mathrm{H}$ & -1.57267100 & -0.88407500 & 1.51527800 & $\mathrm{C}$ & 3.31223600 & 4.00385500 & -1.11659500 \\
\hline $\mathrm{C}$ & -5.60851900 & -3.93222700 & -0.98865200 & $\mathrm{C}$ & 4.41590000 & 3.80698700 & -1.94985800 \\
\hline $\mathrm{C}$ & -4.34967200 & -3.72499400 & -0.42125900 & $\mathrm{C}$ & 4.95312600 & 2.52722000 & -2.08557600 \\
\hline $\mathrm{C}$ & -4.12323900 & -2.65788100 & 0.45630400 & $\mathrm{C}$ & 4.40293000 & 1.45448900 & -1.38003900 \\
\hline $\mathrm{C}$ & -5.18694100 & -1.79613400 & 0.75423900 & $\mathrm{C}$ & 3.30295000 & 1.64497100 & -0.52937700 \\
\hline $\mathrm{C}$ & -6.44664600 & -1.99909000 & 0.18670500 & $\mathrm{C}$ & -2.38794400 & -4.37122600 & 0.70709400 \\
\hline $\mathrm{C}$ & -6.66284200 & -3.06829900 & -0.68624800 & $\mathrm{C}$ & -2.54217300 & -5.70249400 & 0.31260600 \\
\hline $\mathrm{H}$ & -5.76313900 & -4.76329400 & -1.67242300 & $\mathrm{C}$ & -2.22626800 & -6.08849800 & -0.99016900 \\
\hline $\mathrm{H}$ & -3.53027000 & -4.39757700 & -0.66865400 & $\mathrm{C}$ & -1.74853100 & -5.13809200 & -1.89541700 \\
\hline
\end{tabular}




\begin{tabular}{|c|c|c|c|c|c|c|c|}
\hline $\mathrm{C}$ & -1.58637300 & -3.81035400 & -1.50036700 & $\mathrm{C}$ & -2.73171500 & 3.53907800 & 1.16494700 \\
\hline $\mathrm{C}$ & -1.90924000 & -3.40819700 & -0.19435300 & $\mathrm{H}$ & -2.99752700 & 2.70915100 & 0.49516700 \\
\hline $\mathrm{C}$ & -4.57309400 & -1.51097200 & 0.20579900 & $\mathrm{H}$ & -2.72211000 & 3.13574000 & 2.18611700 \\
\hline $\mathrm{C}$ & -5.79625900 & -0.96006900 & -0.17471900 & $\mathrm{C}$ & -3.77625500 & 4.66100700 & 1.03614700 \\
\hline $\mathrm{C}$ & -5.82964100 & 0.16903700 & -0.99858600 & $\mathrm{H}$ & -4.77586900 & 4.26998400 & 1.26305800 \\
\hline $\mathrm{C}$ & -4.63590100 & 0.74240800 & -1.43753100 & $\mathrm{H}$ & -3.56884800 & 5.43951900 & 1.78563100 \\
\hline $\mathrm{C}$ & -3.40890000 & 0.19635500 & -1.04783500 & $\mathrm{C}$ & -1.32391000 & 4.03395700 & 0.80413100 \\
\hline $\mathrm{C}$ & -3.36525300 & -0.93340800 & -0.21995700 & $\mathrm{H}$ & -1.02451700 & 4.79530400 & 1.53825500 \\
\hline $\mathrm{H}$ & 3.25001000 & 0.64707000 & 2.67253900 & $\mathrm{C}$ & -1.29927800 & 4.66457400 & -0.59431900 \\
\hline $\mathrm{H}$ & 2.12721600 & 1.88783800 & 2.09924000 & $\mathrm{H}$ & -1.50622400 & 3.87854800 & -1.33594400 \\
\hline $\mathrm{H}$ & 1.35672500 & -0.97928200 & 2.89764800 & $\mathrm{H}$ & -0.29120100 & 5.04079700 & -0.80690500 \\
\hline $\mathrm{H}$ & 0.99627000 & 0.50951500 & 3.80595200 & $\mathrm{C}$ & -3.75173800 & 5.29146800 & -0.36507600 \\
\hline $\mathrm{H}$ & -2.73064100 & -1.86903400 & 2.53177800 & $\mathrm{H}$ & -4.47126100 & 6.11818600 & -0.42260800 \\
\hline $\mathrm{H}$ & -1.02689700 & -2.37388400 & 2.45535700 & $\mathrm{C}$ & -2.34302300 & 5.78626800 & -0.72866800 \\
\hline $\mathrm{H}$ & -2.11235300 & 0.49256500 & 2.33589100 & $\mathrm{H}$ & -2.06973000 & 6.61818800 & -0.06247800 \\
\hline $\mathrm{H}$ & -1.23548900 & -0.21190300 & 3.69844300 & $\mathrm{H}$ & -2.32946700 & 6.18913300 & -1.74902400 \\
\hline $\mathrm{H}$ & 5.25616100 & 0.32621800 & 1.41044000 & $\mathrm{H}$ & -4.07406900 & 4.54245900 & -1.10352200 \\
\hline $\mathrm{H}$ & 7.06080100 & -1.34395100 & 1.58716700 & $\mathrm{O}$ & -0.34308100 & 2.99972800 & 0.93191500 \\
\hline $\mathrm{H}$ & 6.73465500 & -3.63784000 & 0.67861300 & $\mathrm{H}$ & -0.40933000 & 2.36629400 & 0.17008300 \\
\hline $\mathrm{H}$ & 4.57136600 & -4.24527800 & -0.38919600 & & & & \\
\hline $\mathrm{H}$ & 2.73446900 & -2.57874200 & -0.51046600 & \multirow{2}{*}{\multicolumn{4}{|c|}{$\begin{array}{l}\text { TS8 } \\
\text { imaginary frequency }=-201.69 \mathrm{~cm}^{-1}\end{array}$}} \\
\hline $\mathrm{H}$ & 1.86436300 & 3.10357500 & 0.18270300 & & & & \\
\hline $\mathrm{H}$ & 2.87783000 & 4.99521900 & -1.01316800 & $\mathrm{Ru}$ & 0.26198500 & -0.74268800 & 0.20079600 \\
\hline $\mathrm{H}$ & 4.84707000 & 4.64270400 & -2.49486100 & $\mathrm{P}$ & -2.01688100 & -1.26551700 & -0.18746500 \\
\hline $\mathrm{H}$ & 5.80425300 & 2.35769600 & -2.74008600 & $\mathrm{P}$ & 2.52239300 & -0.18064200 & -0.23581400 \\
\hline $\mathrm{H}$ & 4.83823400 & 0.46735400 & -1.49496400 & $\mathrm{~N}$ & -0.07977200 & 0.58455600 & -1.54263100 \\
\hline $\mathrm{H}$ & -2.63848500 & -4.09463300 & 1.72690900 & $\mathrm{C}$ & -2.38294400 & -0.23617300 & -1.70375300 \\
\hline $\mathrm{H}$ & -2.90858200 & -6.43603600 & 1.02626200 & $\mathrm{C}$ & -1.07906900 & 0.01131300 & -2.46736500 \\
\hline $\mathrm{H}$ & -2.34634900 & -7.12406000 & -1.29719700 & $\mathrm{C}$ & 2.37056100 & 0.22468400 & -2.04944900 \\
\hline $\mathrm{H}$ & -1.49241200 & -5.43090400 & -2.91011500 & $\mathrm{C}$ & 1.12770100 & 1.10633900 & -2.22566900 \\
\hline $\mathrm{H}$ & -1.19199200 & -3.08790600 & -2.20803000 & $\mathrm{C}$ & -3.73643000 & -3.25210900 & -1.23646100 \\
\hline $\mathrm{H}$ & -4.56242700 & -2.40602900 & 0.82266500 & $\mathrm{C}$ & -4.08150600 & -4.54642900 & -1.62272500 \\
\hline $\mathrm{H}$ & -6.72345400 & -1.41576100 & 0.16344600 & $\mathrm{C}$ & -3.17167200 & -5.59365000 & -1.45182100 \\
\hline $\mathrm{H}$ & -6.78352800 & 0.59378700 & -1.30054500 & $\mathrm{C}$ & -1.91952500 & -5.33916100 & -0.89276600 \\
\hline $\mathrm{H}$ & -4.65595400 & 1.61416200 & -2.08678200 & $\mathrm{C}$ & -1.57166800 & -4.04139200 & -0.50855700 \\
\hline $\mathrm{H}$ & -2.47186200 & 0.63887200 & -1.37662900 & $\mathrm{C}$ & -2.47455300 & -2.98277300 & -0.67632100 \\
\hline $\mathrm{C}$ & 0.61283500 & -1.04992800 & -1.86644200 & $\mathrm{C}$ & -3.39050100 & 0.59062700 & 1.38429800 \\
\hline $\mathrm{O}$ & 0.79328700 & -1.36649500 & -2.97476300 & $\mathrm{C}$ & -4.30892100 & 1.00255400 & 2.35003800 \\
\hline $\mathrm{H}$ & -0.29125600 & 0.98459900 & -0.71627100 & $\mathrm{C}$ & -5.12193500 & 0.07073700 & 3.00074200 \\
\hline $\mathrm{H}$ & 0.84823700 & -1.95449400 & 0.58011900 & $\mathrm{C}$ & -5.01166200 & -1.28337800 & 2.68381400 \\
\hline $\mathrm{H}$ & -0.19110700 & 1.26732200 & 1.82788500 & $\mathrm{C}$ & -4.10170000 & -1.70542000 & 1.71153600 \\
\hline
\end{tabular}




\begin{tabular}{|c|c|c|c|c|c|c|c|}
\hline $\mathrm{C}$ & -3.29297200 & -0.77191200 & 1.04657000 & $\mathrm{C}$ & 0.52187500 & -1.94992600 & 1.61232400 \\
\hline $\mathrm{C}$ & 4.72391300 & -1.72011300 & -1.18024000 & $\mathrm{O}$ & 0.68398500 & -2.73267300 & 2.45575500 \\
\hline $\mathrm{C}$ & 5.71227700 & -2.69894000 & -1.05367800 & $\mathrm{H}$ & 0.00056300 & 0.57582800 & 1.64195600 \\
\hline $\mathrm{C}$ & 5.83361300 & -3.42386000 & 0.13243200 & $\mathrm{H}$ & 0.58766600 & -1.92672600 & -0.81302200 \\
\hline $\mathrm{C}$ & 4.96095300 & -3.16841100 & 1.19266400 & $\mathrm{H}$ & -0.58061600 & 1.49494100 & -0.98586800 \\
\hline $\mathrm{C}$ & 3.96848000 & -2.19679800 & 1.06506500 & $\mathrm{C}$ & -0.41630000 & 4.74695400 & -0.47613500 \\
\hline $\mathrm{C}$ & 3.83817400 & -1.45919200 & -0.12315000 & $\mathrm{H}$ & -0.34738800 & 4.83979300 & 0.61871900 \\
\hline $\mathrm{C}$ & 4.66866900 & 1.41538000 & 0.76490900 & $\mathrm{H}$ & 0.57455500 & 4.42822500 & -0.82803700 \\
\hline $\mathrm{C}$ & 5.21541000 & 2.58422400 & 1.29725600 & $\mathrm{C}$ & -0.79099000 & 6.10445500 & -1.09329800 \\
\hline $\mathrm{C}$ & 4.39721900 & 3.68253200 & 1.56725400 & $\mathrm{H}$ & -0.04756000 & 6.86730000 & -0.82421600 \\
\hline $\mathrm{C}$ & 3.02809400 & 3.60641900 & 1.30516600 & $\mathrm{H}$ & -0.76649400 & 6.02388600 & -2.19096000 \\
\hline $\mathrm{C}$ & 2.47268000 & 2.43660200 & 0.78357500 & $\mathrm{C}$ & -1.44685600 & 3.63704600 & -0.78379900 \\
\hline $\mathrm{C}$ & 3.29224700 & 1.32932000 & 0.50097300 & $\mathrm{H}$ & -1.47540200 & 3.53386700 & -1.89521600 \\
\hline $\mathrm{H}$ & -3.13631700 & -0.71456400 & -2.33710800 & $\mathrm{C}$ & -2.85026900 & 4.11634800 & -0.35386200 \\
\hline $\mathrm{H}$ & -2.77204600 & 0.72217600 & -1.34953700 & $\mathrm{H}$ & -2.85388800 & 4.19160600 & 0.74439300 \\
\hline $\mathrm{H}$ & -0.70689300 & -0.92045600 & -2.91451600 & $\mathrm{H}$ & -3.58819500 & 3.34678300 & -0.61786700 \\
\hline $\mathrm{H}$ & -1.27833300 & 0.71695700 & -3.28917400 & $\mathrm{C}$ & -2.19470800 & 6.55102800 & -0.65697300 \\
\hline $\mathrm{H}$ & 3.26358900 & 0.72856300 & -2.43884500 & $\mathrm{H}$ & -2.46439600 & 7.49754600 & -1.14472100 \\
\hline $\mathrm{H}$ & 2.24511400 & -0.72827100 & -2.57565600 & $\mathrm{C}$ & -3.24066600 & 5.46991600 & -0.96919300 \\
\hline $\mathrm{H}$ & 1.33555200 & 2.10181500 & -1.81458000 & $\mathrm{H}$ & -3.32817600 & 5.36169200 & -2.06122200 \\
\hline $\mathrm{H}$ & 0.92860300 & 1.24460900 & -3.29714300 & $\mathrm{H}$ & -4.23070700 & 5.78284800 & -0.61044400 \\
\hline $\mathrm{H}$ & -4.45920200 & -2.44993800 & -1.36024600 & $\mathrm{H}$ & -2.19038300 & 6.74635800 & 0.42600200 \\
\hline $\mathrm{H}$ & -5.05977400 & -4.73778200 & -2.05561100 & $\mathrm{O}$ & -1.11755600 & 2.42615800 & -0.18004300 \\
\hline $\mathrm{H}$ & -3.44034200 & -6.60256900 & -1.75339400 & $\mathrm{H}$ & -0.33892300 & 1.03539400 & 1.11327300 \\
\hline $\mathrm{H}$ & -1.20742900 & -6.14844500 & -0.75601800 & & & & \\
\hline $\mathrm{H}$ & -0.59257300 & -3.84739700 & -0.08509000 & 16 & & & \\
\hline $\mathrm{H}$ & -2.74038200 & 1.32558200 & 0.90610900 & $\mathrm{Ru}$ & 0.39245600 & -0.68053600 & 0.25411900 \\
\hline $\mathrm{H}$ & -4.38271100 & 2.05843400 & 2.59713100 & $\mathrm{P}$ & -1.74986000 & -1.59250500 & -0.17741100 \\
\hline $\mathrm{H}$ & -5.83257600 & 0.39794700 & 3.75535000 & $\mathrm{P}$ & 2.51732800 & 0.25363100 & -0.21210200 \\
\hline $\mathrm{H}$ & -5.63322300 & -2.01669400 & 3.19113200 & $\mathrm{~N}$ & -0.21605600 & 0.66160700 & -1.36761900 \\
\hline $\mathrm{H}$ & -4.02871700 & -2.76180300 & 1.47380500 & $\mathrm{C}$ & -2.31559400 & -0.56723100 & -1.63016700 \\
\hline $\mathrm{H}$ & 4.65075100 & -1.16170300 & -2.10817600 & $\mathrm{C}$ & -1.07357900 & -0.01905500 & -2.34108500 \\
\hline $\mathrm{H}$ & 6.38709600 & -2.89192000 & -1.88338100 & $\mathrm{C}$ & 2.23676400 & 0.77457400 & -1.97547900 \\
\hline $\mathrm{H}$ & 6.60241400 & -4.18558900 & 0.22983600 & $\mathrm{C}$ & 0.84933100 & 1.44186100 & -2.01079900 \\
\hline $\mathrm{H}$ & 5.04634000 & -3.73065100 & 2.11845500 & $\mathrm{C}$ & -3.06891800 & -3.80698800 & -1.34663800 \\
\hline $\mathrm{H}$ & 3.28827300 & -2.01874700 & 1.89191400 & $\mathrm{C}$ & -3.16525100 & -5.11959400 & -1.80633600 \\
\hline $\mathrm{H}$ & 5.31684500 & 0.56925300 & 0.56024100 & $\mathrm{C}$ & -2.07411900 & -5.98485500 & -1.68612400 \\
\hline $\mathrm{H}$ & 6.28175300 & 2.63353400 & 1.50204100 & $\mathrm{C}$ & -0.89100700 & -5.53066700 & -1.10383400 \\
\hline $\mathrm{H}$ & 4.82425000 & 4.59162700 & 1.98249900 & $\mathrm{C}$ & -0.79262200 & -4.21393200 & -0.64614600 \\
\hline $\mathrm{H}$ & 2.38150100 & 4.45518200 & 1.50973200 & $\mathrm{C}$ & -1.87889400 & -3.33643700 & -0.76285200 \\
\hline $\mathrm{H}$ & 1.40264200 & 2.40132700 & 0.59394400 & $\mathrm{C}$ & -3.47940300 & -0.14645800 & 1.48950300 \\
\hline
\end{tabular}




\begin{tabular}{|c|c|c|c|c|c|c|c|}
\hline $\mathrm{C}$ & -4.45120000 & 0.01461500 & 2.47697000 & $\mathrm{H}$ & 5.88804800 & 3.46599400 & 1.63872100 \\
\hline $\mathrm{C}$ & -5.03991700 & -1.10004300 & 3.07994200 & $\mathrm{H}$ & 4.18166300 & 5.14155500 & 2.32542700 \\
\hline $\mathrm{C}$ & -4.64791600 & -2.38097800 & 2.69171200 & $\mathrm{H}$ & 1.76859800 & 4.67676200 & 1.92150600 \\
\hline $\mathrm{C}$ & -3.68086400 & -2.54996600 & 1.69749100 & $\mathrm{H}$ & 1.06660800 & 2.57494000 & 0.86670000 \\
\hline $\mathrm{C}$ & -3.09431400 & -1.43607300 & 1.07931800 & $\mathrm{C}$ & 0.88704100 & -1.89645800 & 1.60355000 \\
\hline $\mathrm{C}$ & 4.89840900 & -0.84098500 & -1.31738600 & $\mathrm{O}$ & 1.20513200 & -2.68500600 & 2.39829300 \\
\hline $\mathrm{C}$ & 6.03057600 & -1.65869900 & -1.29429500 & $\mathrm{H}$ & 0.14195900 & 0.73312900 & 1.58667300 \\
\hline $\mathrm{C}$ & 6.30138000 & -2.44977400 & -0.17710200 & $\mathrm{H}$ & 0.88889100 & -1.72053800 & -0.84691800 \\
\hline $\mathrm{C}$ & 5.43395300 & -2.42269400 & 0.91731600 & $\mathrm{H}$ & -1.15784600 & 1.81863600 & -0.50882400 \\
\hline $\mathrm{C}$ & 4.29900700 & -1.61215600 & 0.89282100 & $\mathrm{C}$ & -1.33737400 & 4.66522600 & -0.89192300 \\
\hline $\mathrm{C}$ & 4.01785000 & -0.80934000 & -0.22474700 & $\mathrm{H}$ & -1.02786300 & 5.00415300 & 0.10747500 \\
\hline $\mathrm{C}$ & 4.44068700 & 2.08081100 & 0.85389900 & $\mathrm{H}$ & -0.42756400 & 4.33591800 & -1.41020800 \\
\hline $\mathrm{C}$ & 4.83256300 & 3.27407300 & 1.46380800 & $\mathrm{C}$ & -2.00181200 & 5.82332300 & -1.65434200 \\
\hline $\mathrm{C}$ & 3.87556600 & 4.21373800 & 1.84941000 & $\mathrm{H}$ & -1.30722000 & 6.66915600 & -1.73879200 \\
\hline $\mathrm{C}$ & 2.52263600 & 3.95279100 & 1.62463600 & $\mathrm{H}$ & -2.22478300 & 5.50234900 & -2.68322600 \\
\hline $\mathrm{C}$ & 2.12581100 & 2.75644300 & 1.02523800 & $\mathrm{C}$ & -2.28812000 & 3.46968000 & -0.70701300 \\
\hline $\mathrm{C}$ & 3.08280300 & 1.80667000 & 0.62611100 & $\mathrm{H}$ & -2.54190400 & 3.07623700 & -1.70936700 \\
\hline $\mathrm{H}$ & -2.95166600 & -1.15223400 & -2.30218700 & $\mathrm{C}$ & -3.58868300 & 3.92193300 & -0.03156500 \\
\hline $\mathrm{H}$ & -2.90326000 & 0.26448700 & -1.23035300 & $\mathrm{H}$ & -3.34477100 & 4.23344300 & 0.99455400 \\
\hline $\mathrm{H}$ & -0.56298200 & -0.84442300 & -2.87135800 & $\mathrm{H}$ & -4.26879800 & 3.06473500 & 0.05213800 \\
\hline $\mathrm{H}$ & -1.40951600 & 0.68967900 & -3.11828700 & $\mathrm{C}$ & -3.30545500 & 6.26654300 & -0.97255800 \\
\hline $\mathrm{H}$ & 3.02146800 & 1.44909500 & -2.34159100 & $\mathrm{H}$ & -3.79129200 & 7.05950000 & -1.55605000 \\
\hline $\mathrm{H}$ & 2.23907800 & -0.13939500 & -2.58003600 & $\mathrm{C}$ & -4.26350700 & 5.07996600 & -0.78411400 \\
\hline $\mathrm{H}$ & 0.93796300 & 2.41882300 & -1.51214700 & $\mathrm{H}$ & -4.59638800 & 4.72691300 & -1.77189800 \\
\hline $\mathrm{H}$ & 0.59246100 & 1.66153700 & -3.05858900 & $\mathrm{H}$ & -5.16761600 & 5.40023900 & -0.25028300 \\
\hline $\mathrm{H}$ & -3.92968300 & -3.14909300 & -1.43318000 & $\mathrm{H}$ & -3.06919500 & 6.69996300 & 0.01066400 \\
\hline $\mathrm{H}$ & -4.09091600 & -5.46697600 & -2.25756100 & $\mathrm{O}$ & -1.69953300 & 2.44649200 & 0.07952500 \\
\hline $\mathrm{H}$ & -2.14865600 & -7.00787700 & -2.04531100 & $\mathrm{H}$ & -0.60006600 & 0.52405900 & 1.46269700 \\
\hline $\mathrm{H}$ & -0.03870500 & -6.19764800 & -1.00643400 & & & & \\
\hline $\mathrm{H}$ & 0.13329700 & -3.86208500 & -0.20532000 & \multirow{2}{*}{\multicolumn{4}{|c|}{$\begin{array}{l}\text { TS9 } \\
\text { imaginary frequency }=-1207.94 \mathrm{~cm}^{-1}\end{array}$}} \\
\hline $\mathrm{H}$ & -3.01446800 & 0.73636400 & 1.05286500 & & & & \\
\hline $\mathrm{H}$ & -4.74313200 & 1.01693800 & 2.77941500 & $\mathrm{Ru}$ & -0.00230100 & -0.23139700 & 0.12299800 \\
\hline $\mathrm{H}$ & -5.79369900 & -0.96978400 & 3.85201700 & $\mathrm{P}$ & 2.32701800 & -0.04203100 & 0.43356300 \\
\hline $\mathrm{H}$ & -5.09269900 & -3.25481600 & 3.16076200 & $\mathrm{P}$ & -2.33392200 & -0.05447300 & 0.43150700 \\
\hline $\mathrm{H}$ & -3.38841700 & -3.55334300 & 1.40555000 & $\mathrm{~N}$ & -0.00581700 & 0.05363600 & 2.31884300 \\
\hline $\mathrm{H}$ & 4.70683400 & -0.22789100 & -2.19248500 & $\mathrm{C}$ & 2.44841900 & -0.05286600 & 2.30093100 \\
\hline $\mathrm{H}$ & 6.69963200 & -1.67517100 & -2.15059700 & $\mathrm{C}$ & 1.20258100 & 0.63075700 & 2.87604700 \\
\hline $\mathrm{H}$ & 7.18183300 & -3.08651300 & -0.16007700 & $\mathrm{C}$ & -2.45542800 & -0.07956100 & 2.29483900 \\
\hline $\mathrm{H}$ & 5.63500200 & -3.03901700 & 1.78930800 & $\mathrm{C}$ & -1.21976400 & 0.61375300 & 2.88073900 \\
\hline $\mathrm{H}$ & 3.62496300 & -1.61387500 & 1.74330400 & $\mathrm{C}$ & 3.13866500 & -2.72188700 & 0.31463200 \\
\hline $\mathrm{H}$ & 5.19737700 & 1.36048100 & 0.56025600 & $\mathrm{C}$ & 3.93143900 & -3.79802400 & -0.08023200 \\
\hline
\end{tabular}




\begin{tabular}{|c|c|c|c|c|c|c|c|}
\hline $\mathrm{C}$ & 5.03469300 & -3.59045800 & -0.91242000 & $\mathrm{H}$ & -3.37942600 & -4.72009000 & -0.85770700 \\
\hline $\mathrm{C}$ & 5.33665100 & -2.30000300 & -1.34576600 & $\mathrm{H}$ & -5.82340500 & -4.34079200 & -1.12948800 \\
\hline $\mathrm{C}$ & 4.55013100 & -1.21788900 & -0.94162200 & $\mathrm{H}$ & -6.76872100 & -2.07106800 & -0.75632700 \\
\hline $\mathrm{C}$ & 3.44698200 & -1.41387900 & -0.09856800 & $\mathrm{H}$ & -5.29266000 & -0.20029400 & -0.11643700 \\
\hline $\mathrm{C}$ & 2.65275500 & 2.32630200 & -1.05903000 & $\mathrm{H}$ & -4.41698000 & 1.81325100 & 1.60143500 \\
\hline $\mathrm{C}$ & 3.31685300 & 3.48526000 & -1.46797600 & $\mathrm{H}$ & -5.48181600 & 3.84633400 & 0.72188600 \\
\hline $\mathrm{C}$ & 4.54751600 & 3.82482600 & -0.90538900 & $\mathrm{H}$ & -4.87428900 & 4.70790700 & -1.53090000 \\
\hline $\mathrm{C}$ & 5.11861400 & 2.99756400 & 0.06526300 & $\mathrm{H}$ & -3.16501600 & 3.51113600 & -2.88808100 \\
\hline $\mathrm{C}$ & 4.45881300 & 1.83724000 & 0.46961400 & $\mathrm{H}$ & -2.05792200 & 1.49137800 & -1.98779300 \\
\hline $\mathrm{C}$ & 3.21477500 & 1.48916500 & -0.08533900 & $\mathrm{C}$ & 0.00616500 & -0.65867400 & -1.69893800 \\
\hline $\mathrm{C}$ & -2.96351500 & -2.67700600 & -0.32467400 & $\mathrm{O}$ & 0.00727900 & -0.89389400 & -2.83879200 \\
\hline $\mathrm{C}$ & -3.80132800 & -3.73325700 & -0.68647800 & $\mathrm{H}$ & -0.03319300 & 1.31689400 & -0.30938300 \\
\hline $\mathrm{C}$ & -5.17225100 & -3.52002700 & -0.84031600 & $\mathrm{H}$ & 0.00650300 & -1.30551500 & 1.76748700 \\
\hline $\mathrm{C}$ & -5.70321600 & -2.24548600 & -0.63072400 & $\mathrm{H}$ & 0.01533800 & -1.94698900 & 1.06032000 \\
\hline $\mathrm{C}$ & -4.86855400 & -1.18873500 & -0.26461400 & & & & \\
\hline $\mathrm{C}$ & -3.48960200 & -1.39458500 & -0.10156000 & a3 & & & \\
\hline $\mathrm{C}$ & -4.13067300 & 2.17287800 & 0.61792700 & $\mathrm{C}$ & 1.54980200 & -2.00374400 & 0.03725100 \\
\hline $\mathrm{C}$ & -4.74087700 & 3.32698400 & 0.11973000 & $\mathrm{C}$ & 1.05702700 & -0.69385700 & 0.02264000 \\
\hline $\mathrm{C}$ & -4.39961400 & 3.81028400 & -1.14360200 & $\mathrm{C}$ & 1.97085200 & 0.37437300 & -0.00535100 \\
\hline $\mathrm{C}$ & -3.44043300 & 3.13837500 & -1.90515000 & $\mathrm{C}$ & 3.35163900 & 0.11522500 & -0.02837700 \\
\hline $\mathrm{C}$ & -2.82232800 & 1.99373200 & -1.40316200 & $\mathrm{C}$ & 3.82847800 & -1.19228600 & 0.00202500 \\
\hline $\mathrm{C}$ & -3.16394700 & 1.49129700 & -0.13684300 & $\mathrm{C}$ & 2.92231000 & -2.25879700 & 0.03650400 \\
\hline $\mathrm{H}$ & 2.47602500 & -1.10391300 & 2.60943000 & $\mathrm{H}$ & 0.84317400 & -2.83135500 & 0.04299900 \\
\hline $\mathrm{H}$ & 3.36701800 & 0.42448400 & 2.65649700 & $\mathrm{H}$ & -0.03389600 & -0.51895600 & -0.02022100 \\
\hline $\mathrm{H}$ & 1.21791600 & 0.52264300 & 3.97641700 & $\mathrm{H}$ & 4.03096400 & 0.96195000 & -0.07098100 \\
\hline $\mathrm{H}$ & 1.25765700 & 1.71770300 & 2.66898800 & $\mathrm{H}$ & 4.90005300 & -1.38426900 & -0.00459400 \\
\hline $\mathrm{H}$ & -3.38506800 & 0.35609000 & 2.67444400 & $\mathrm{H}$ & 3.28969800 & -3.28387900 & 0.05519200 \\
\hline $\mathrm{H}$ & -2.45490000 & -1.14023400 & 2.57174900 & $\mathrm{C}$ & 1.52650900 & 1.81448200 & -0.02987300 \\
\hline $\mathrm{H}$ & -1.28612600 & 1.70217700 & 2.68427800 & $\mathrm{O}$ & 2.31198500 & 2.68594500 & -0.40040200 \\
\hline $\mathrm{H}$ & -1.23505700 & 0.49437000 & 3.97997900 & $\mathrm{C}$ & 0.13566600 & 2.14080000 & 0.44310300 \\
\hline $\mathrm{H}$ & 2.26278300 & -2.90112700 & 0.93211500 & $\mathrm{H}$ & -0.65756900 & 1.52036100 & -0.04355600 \\
\hline $\mathrm{H}$ & 3.68072400 & -4.80148900 & 0.25371900 & $\mathrm{H}$ & 0.05631000 & 1.89731600 & 1.51291100 \\
\hline $\mathrm{H}$ & 5.64816900 & -4.43075000 & -1.22636800 & $\mathrm{H}$ & -0.04099600 & 3.21228300 & 0.30753900 \\
\hline $\mathrm{H}$ & 6.18659000 & -2.12887100 & -2.00127400 & $\mathrm{C}$ & -3.02028400 & -0.23496000 & -0.07280800 \\
\hline $\mathrm{H}$ & 4.80124000 & -0.22084000 & -1.28732200 & $\mathrm{C}$ & -3.15301700 & -0.02382100 & 1.47014900 \\
\hline $\mathrm{H}$ & 1.68609300 & 2.07158600 & -1.48067700 & $\mathrm{H}$ & -2.91360900 & 1.01882000 & 1.71842600 \\
\hline $\mathrm{H}$ & 2.86668300 & 4.12449100 & -2.22271800 & $\mathrm{H}$ & -4.15511300 & -0.24984100 & 1.87241800 \\
\hline $\mathrm{H}$ & 5.06090500 & 4.72978900 & -1.21954800 & $\mathrm{H}$ & -2.42445900 & -0.66298000 & 1.98673300 \\
\hline $\mathrm{H}$ & 6.07766600 & 3.25506000 & 0.50701600 & $\mathrm{C}$ & -4.07059500 & 0.68623500 & -0.77189100 \\
\hline $\mathrm{H}$ & 4.92457500 & 1.19552200 & 1.21267900 & $\mathrm{H}$ & -3.83414200 & 1.73565600 & -0.55156200 \\
\hline $\mathrm{H}$ & -1.89511600 & -2.84060900 & -0.21784400 & $\mathrm{H}$ & -3.99385100 & 0.55342800 & -1.85887400 \\
\hline
\end{tabular}




$\begin{array}{lrrr}\mathrm{H} & -5.11384500 & 0.49515200 & -0.46872800 \\ \mathrm{C} & -3.40544800 & -1.71716400 & -0.38328800 \\ \mathrm{H} & -3.31187800 & -1.89080000 & -1.46315800 \\ \mathrm{H} & -2.69620200 & -2.38781400 & 0.12050500 \\ \mathrm{H} & -4.42609500 & -1.99573600 & -0.07080700 \\ \mathrm{O} & -1.77150600 & 0.03552300 & -0.52269300\end{array}$

\section{TS10}

imaginary frequency $=-1070.18 \mathrm{~cm}^{-1}$

C

C

C

C

C

C

H

H

H

H

H

C

O

C

H

$\mathrm{H}$

H

C

C

H

H

$\mathrm{H}$

C

H

$\mathrm{H}$

$\mathrm{H}$

C

H

$\mathrm{H}$

H

O

$\begin{array}{rrrr}2.26209400 & -1.97329700 & 0.09449500 & \mathrm{H} \\ 1.39933900 & -0.87440300 & 0.13363400 & \mathrm{H} \\ 1.91631000 & 0.42871600 & 0.04388900 & \mathrm{H} \\ 3.29939400 & 0.60094100 & -0.10909700 & \mathrm{C} \\ 4.16005400 & -0.49598700 & -0.13189200 & \mathrm{O} \\ 3.64254900 & -1.79094500 & -0.02604700 & \mathrm{C} \\ 1.85161600 & -2.98022700 & 0.14911800 & \mathrm{H} \\ 0.32025400 & -1.01563900 & 0.17474000 & \mathrm{H}\end{array}$

$3.67229300 \quad 1.61592800 \quad-0.21494100$

$\begin{array}{lll}5.23355300 & -0.34552800 & -0.23807200\end{array}$

$\begin{array}{lll}4.30977900 & -2.65116400 & -0.04986700\end{array}$

$\begin{array}{lll}1.03126200 & 1.66675700 & 0.07862600\end{array}$

$\begin{array}{lll}1.44426000 & 2.68513200 & -0.50368400\end{array}$

$\begin{array}{lll}-0.24818700 & 1.53410400 & 0.75034700\end{array}$

$\begin{array}{lll}-1.07115500 & 0.70354700 & 0.04844400\end{array}$

$\begin{array}{lll}-0.18820600 & 0.96262300 & 1.68369900\end{array}$

$\begin{array}{lll}-0.73018400 & 2.50525900 & 0.89937600\end{array}$

$\begin{array}{lll}-3.05879500 & -0.34333300 & -0.10445200\end{array}$

$\begin{array}{lll}-3.17235000 & -0.55175000 & 1.43035200\end{array}$

$\begin{array}{lll}-2.75242400 & 0.30977900 & 1.96264400\end{array}$

$-4.21161900-0.68802300$

1.76746200

$-2.59531300-1.43802900$

1.72258700

$-3.85861200$

0.92647300

$-0.50073600$

$-3.43710800$

1.80597600

0.00057000

$-3.77023000$

1.08736700

$-1.58192400$

$-4.92701900$

0.86164400

$-0.24202700$

$-3.68360700-1.56769300$

$-0.81591800$

$\begin{array}{lll}-3.60768500 & -1.43737100 & -1.90224800\end{array}$

$-3.12207900 \quad-2.47183200 \quad-0.55009900$

$\begin{array}{lll}-4.74175000 & -1.72250700 & -0.55345000\end{array}$

$\begin{array}{lll}-1.74043900 & -0.24254400 & -0.51321700\end{array}$ a4

$\mathrm{C}$

C

C

C

C

C

$\mathrm{H}$

$\mathrm{H}$

$\mathrm{H}$

$\mathrm{H}$

$\mathrm{H}$

C

$\mathrm{O}$

C

$\mathrm{H}$

$\mathrm{H}$

\section{TS11}

imaginary frequency $=-188.25 \mathrm{~cm}^{-1}$

$\begin{array}{lrrr}\mathrm{C} & -3.28312500 & 1.48130800 & 0.23002400 \\ \mathrm{C} & -2.02773800 & 0.98745200 & -0.13654800 \\ \mathrm{C} & -1.84001900 & -0.39391100 & -0.31151800 \\ \mathrm{C} & -2.92398400 & -1.25838000 & -0.09349900 \\ \mathrm{C} & -4.18012500 & -0.76079400 & 0.24986800 \\ \mathrm{C} & -4.36596400 & 0.61635000 & 0.40991800 \\ \mathrm{H} & -3.41391000 & 2.55175700 & 0.38082600 \\ \mathrm{H} & -1.17380100 & 1.65945800 & -0.22539600 \\ \mathrm{H} & -2.74314600 & -2.32445700 & -0.19581900 \\ \mathrm{H} & -5.01492600 & -1.44413700 & 0.40075400 \\ \mathrm{H} & -5.34441400 & 1.00976600 & 0.68171500 \\ \mathrm{C} & -0.50601100 & -1.00628900 & -0.70293500 \\ \mathrm{O} & -0.32615000 & -2.21592600 & -0.42167100 \\ \mathrm{C} & 0.47318900 & -0.16353800 & -1.32340000 \\ \mathrm{H} & 0.10930800 & 0.69928900 & -1.87407500 \\ \mathrm{H} & 1.23747400 & -0.73147300 & -1.85120600 \\ \mathrm{C} & 2.34950100 & -1.03827200 & 1.24817800 \\ \mathrm{H} & 1.81666200 & -1.75184900 & 0.60877500 \\ \mathrm{H} & 2.41750200 & -1.49704400 & 2.24683300 \\ \mathrm{C} & 1.54514100 & 0.26688300 & 1.32970600 \\ \mathrm{H} & 0.51827800 & 0.07884200 & 1.66000100 \\ & & & \\ & & \end{array}$


C

$\mathrm{H}$

$\mathrm{H}$

C

$\mathrm{H}$

$\mathrm{H}$

C

C

$\mathrm{H}$

$\mathrm{H}$

O

\section{ab1}

$\mathrm{C}$

C

C

C

C

C

$\mathrm{H}$

$\mathrm{H}$

$\mathrm{H}$

$\mathrm{H}$

$\mathrm{H}$

C

O

C

$\mathrm{H}$

$\mathrm{H}$

C

$\mathrm{H}$

$\mathrm{H}$

C

$\mathrm{H}$

$\mathrm{H}$

C

$\mathrm{H}$

$\mathrm{H}$

C

$\mathrm{H}$

$\begin{array}{rrr}1.99880400 & 0.92673700 & 2.09152800 \\ 3.76036500 & -0.78793400 & 0.69521700 \\ 4.33269600 & -1.72713800 & 0.65996900 \\ 4.30690200 & -0.11438900 & 1.37630500 \\ 3.70053300 & -0.15497700 & -0.70289500 \\ 3.26339700 & -0.88347200 & -1.39685400 \\ 4.71837000 & 0.05101700 & -1.07047300 \\ 1.50708000 & 1.10854000 & 0.03402400 \\ 2.87239600 & 1.14005800 & -0.70721600 \\ 3.44866700 & 1.92653300 & -0.18946600 \\ 2.70835600 & 1.50797200 & -1.72730100 \\ 0.86694400 & 2.19637800 & 0.06046500\end{array}$

3.33867100

2.06094000

1.87881400

2.97143600

4.24462300

4.43102600

3.48127000

1.16689600

2.79150800

5.09129200

5.42551200

0.52389600

0.34066600

$-0.55931200$

$-0.16851300$

$-1.27405000$

$-2.68589000$

$-2.23286800$

$-2.90793700$

$-1.68771800$

$-0.75568700$

$-2.10273200$

$-3.99243400$

$-4.69497900$

$-4.48411100$

$-3.72807600$

$-3.37613500$
$-1.47628100$

$-0.98866000$

0.39388500

1.26571400

0.76723600

$-0.61114200$

$-2.54478700$

$-1.63501800$

2.33172800

1.44650200

$-1.00462600$

0.97750400

2.18921100

0.08467200

$-0.64791300$

0.73914000

1.12046400

1.87839300

1.62761000

$-0.02794400$

0.34897200

$-0.74341400$

0.60640200

1.43698400

$-0.07693200$

$-0.14796900$

$0.57109800 \quad-1.60821400$
$\mathrm{H}$

C

C

$\mathrm{H}$

$\mathrm{H}$

O

ab2

$\mathrm{C}$

C

C

C

C

C

$\mathrm{H}$

$\mathrm{H}$

$\mathrm{H}$

$\mathrm{H}$

$\mathrm{H}$

C

O

C

H

H

$\mathrm{H}$

C

C

$\mathrm{H}$

$\mathrm{H}$

$\mathrm{H}$

C

$\mathrm{H}$

$\mathrm{H}$

$\mathrm{H}$

C

$\mathrm{H}$

H

$\mathrm{H}$

O

C

H

$\begin{array}{lll}-4.67309700 & -0.55711400 & -1.24739600 \\ -1.33563300 & -0.88708100 & 0.04099600 \\ -2.69365900 & -1.27141800 & -0.66460900 \\ -3.12327300 & -2.05610700 & -0.02460500 \\ -2.45943900 & -1.75355300 & -1.62469500 \\ -0.62863500 & -1.96336800 & 0.33692800\end{array}$

\begin{tabular}{|c|c|c|}
\hline 2.78590500 & -2.28779000 & 0.94427300 \\
\hline 1.97737500 & -1.39132600 & 0.23936600 \\
\hline 2.55362000 & -0.28172700 & -0.40372900 \\
\hline 3.94398000 & -0.09861000 & -0.32266100 \\
\hline 4.74673400 & -0.99686100 & 0.37476100 \\
\hline 4.16720000 & -2.09861200 & 1.01280900 \\
\hline 2.32660300 & -3.13803800 & 1.44305900 \\
\hline 0.90409300 & -1.56527600 & 0.21284100 \\
\hline 4.36499700 & 0.76822700 & -0.82272900 \\
\hline 5.82266100 & -0.84068400 & 0.42546700 \\
\hline 4.78973000 & -2.80329400 & 1.56081400 \\
\hline 1.76038500 & 0.73376500 & -1.20372500 \\
\hline 2.35669800 & 1.70287500 & -1.68 \\
\hline 0.28719700 & 0.54160800 & -1.37088700 \\
\hline 1.66690200 & -0.67146700 & 0.17408900 \\
\hline-0.00215900 & -0.50078300 & -1.24795800 \\
\hline 0.01538500 & 0.85942200 & -2.3828 \\
\hline 2.67522000 & -2.35747100 & -0.09475500 \\
\hline-3.92478500 & -1.82548500 & 0.64077100 \\
\hline-4.01226300 & -0.74770100 & 0.46769100 \\
\hline-4.84870600 & -2.31741600 & 0.30357400 \\
\hline-3.81766800 & -1.98860900 & 1.72030500 \\
\hline-2.82454300 & -2.11311300 & -1.61203400 \\
\hline-2.83211500 & -1.03289900 & -1.79119900 \\
\hline-1.96710400 & -2.54161100 & -2.14665700 \\
\hline-3.74309000 & -2.56161200 & -2.01759200 \\
\hline-2.48067500 & -3.85253200 & 0.19589600 \\
\hline-1.57426000 & -4.21622400 & -0.30300000 \\
\hline-2.35974000 & -4.01424900 & 1.27399800 \\
\hline-3.33390500 & -4.44990400 & -0.15337700 \\
\hline-1.52894700 & -1.68903000 & 0.39205200 \\
\hline-1.73284900 & 3.66661400 & -0.04336000 \\
\hline-2.72309300 & 3.19585100 & -0.00792400 \\
\hline
\end{tabular}




\begin{tabular}{|c|c|c|c|c|c|c|c|}
\hline $\mathrm{H}$ & -1.85832400 & 4.67703000 & -0.46245000 & $\mathrm{H}$ & 2.85575000 & 1.53989700 & -1.85732700 \\
\hline $\mathrm{C}$ & -0.84693200 & 2.80481200 & -0.95400000 & $\mathrm{H}$ & 1.64373300 & 2.78158100 & -2.21191600 \\
\hline $\mathrm{H}$ & -1.31727100 & 2.71033400 & -1.94227100 & $\mathrm{H}$ & 3.33179500 & 3.26356800 & -1.90998600 \\
\hline $\mathrm{H}$ & 0.13620300 & 3.27657800 & -1.10066800 & $\mathrm{C}$ & 1.61336600 & 4.02765000 & 0.22208100 \\
\hline $\mathrm{C}$ & -1.16537900 & 3.74982700 & 1.38267200 & $\mathrm{H}$ & 0.69263700 & 4.19041900 & -0.35190300 \\
\hline $\mathrm{H}$ & -1.84089200 & 4.32906900 & 2.03108000 & $\mathrm{H}$ & 1.35744500 & 4.07943300 & 1.28769900 \\
\hline $\mathrm{H}$ & -0.20842900 & 4.29693600 & 1.36114400 & $\mathrm{H}$ & 2.31518800 & 4.84274000 & -0.00840800 \\
\hline $\mathrm{C}$ & -0.93150600 & 2.34713200 & 1.96532700 & $\mathrm{O}$ & 1.24135400 & 1.67409300 & 0.22395700 \\
\hline $\mathrm{H}$ & -1.89256000 & 1.82679800 & 2.05431000 & $\mathrm{C}$ & 2.19843200 & -3.45996600 & -0.00806200 \\
\hline $\mathrm{H}$ & -0.49681900 & 2.42113200 & 2.97370000 & $\mathrm{H}$ & 3.13346900 & -2.88671600 & -0.02489800 \\
\hline $\mathrm{C}$ & -0.68771500 & 1.37139300 & -0.36538800 & $\mathrm{H}$ & 2.42144700 & -4.46847700 & -0.38865900 \\
\hline $\mathrm{C}$ & -0.03192200 & 1.50777300 & 1.04705300 & $\mathrm{C}$ & 1.19558700 & -2.74757600 & -0.92681800 \\
\hline $\mathrm{H}$ & 0.96400200 & 1.97474200 & 0.98213800 & $\mathrm{H}$ & 1.62414600 & -2.64641900 & -1.93302400 \\
\hline $\mathrm{H}$ & 0.10280100 & 0.50769500 & 1.47690800 & $\mathrm{H}$ & 0.27167300 & -3.33708000 & -1.02211800 \\
\hline $\mathrm{O}$ & -1.88480100 & 0.73985200 & -0.29614600 & $\mathrm{C}$ & 1.68292100 & -3.53824700 & 1.43726500 \\
\hline & & & & $\mathrm{H}$ & 2.43713200 & -4.00611200 & 2.08834400 \\
\hline & & & & $\mathrm{H}$ & 0.79387900 & -4.18916400 & 1.47125600 \\
\hline & lency $=-65$ & $8.21 \mathrm{~cm}^{-1}$ & & $\mathrm{C}$ & 1.30900400 & -2.14346100 & 1.96144100 \\
\hline $\mathrm{C}$ & -2.89197100 & 1.99081700 & 0.90082500 & $\mathrm{H}$ & 2.20830400 & -1.51747300 & 1.99963200 \\
\hline $\mathrm{C}$ & -2.02040400 & 1.14575700 & 0.20650200 & $\mathrm{H}$ & 0.91145800 & -2.21472800 & 2.98482600 \\
\hline $\mathrm{C}$ & -2.51588500 & -0.02984300 & -0.38581200 & $\mathrm{C}$ & 0.86301200 & -1.32626900 & -0.40598700 \\
\hline $\mathrm{C}$ & -3.88271800 & -0.33224100 & -0.26633200 & $\mathrm{C}$ & 0.29335100 & -1.45241700 & 1.04003600 \\
\hline $\mathrm{C}$ & -4.74589700 & 0.51674600 & 0.41994300 & $\mathrm{H}$ & -0.64900800 & -2.02270600 & 1.04169600 \\
\hline $\mathrm{C}$ & -4.24974100 & 1.68580400 & 1.00736600 & $\mathrm{H}$ & 0.07482800 & -0.44933000 & 1.42210500 \\
\hline $\mathrm{H}$ & -2.49805300 & 2.89387600 & 1.36127400 & $\mathrm{O}$ & 2.00195100 & -0.55020700 & -0.41352100 \\
\hline $\mathrm{H}$ & -0.96341300 & 1.41081800 & 0.15319000 & & & & \\
\hline $\mathrm{H}$ & -4.23786300 & -1.24888300 & -0.72740100 & ab3 & & & \\
\hline $\mathrm{H}$ & -5.80300900 & 0.27087800 & 0.50116000 & $\mathrm{C}$ & -3.20577300 & 1.17103500 & 0.77790400 \\
\hline $\mathrm{H}$ & -4.91997300 & 2.35277500 & 1.54647500 & $\mathrm{C}$ & -2.15008600 & 0.53463600 & 0.11623600 \\
\hline $\mathrm{C}$ & -1.65203400 & -0.99767200 & -1.16571400 & $\mathrm{C}$ & -2.32278500 & -0.78037600 & -0.35226100 \\
\hline $\mathrm{O}$ & -2.15653900 & -2.03933300 & -1.58831700 & $\mathrm{C}$ & -3.55149200 & -1.43147800 & -0.14561700 \\
\hline $\mathrm{C}$ & -0.20658400 & -0.66324800 & -1.40148100 & $\mathrm{C}$ & -4.59784200 & -0.78773100 & 0.50719500 \\
\hline $\mathrm{H}$ & 1.66170200 & 0.57813300 & -0.05868900 & $\mathrm{C}$ & -4.42557100 & 0.52169800 & 0.97159200 \\
\hline $\mathrm{H}$ & -0.02877100 & 0.40979900 & -1.34027400 & $\mathrm{H}$ & -3.06293000 & 2.18590600 & 1.14204800 \\
\hline $\mathrm{H}$ & 0.05098500 & -1.00275200 & -2.41084900 & $\mathrm{H}$ & -1.20103400 & 1.06993300 & -0.00520400 \\
\hline $\mathrm{C}$ & 2.19358300 & 2.63743200 & -0.10718200 & $\mathrm{H}$ & -3.65416900 & -2.44834000 & -0.51245600 \\
\hline $\mathrm{C}$ & 3.48273100 & 2.40799400 & 0.72142700 & $\mathrm{H}$ & -5.54592000 & -1.30066200 & 0.65743000 \\
\hline $\mathrm{H}$ & 3.88305100 & 1.41077100 & 0.50634400 & $\mathrm{H}$ & -5.24102600 & 1.02960000 & 1.48357400 \\
\hline $\mathrm{H}$ & 4.26466800 & 3.15338300 & 0.51110600 & $\mathrm{C}$ & -1.24403900 & -1.54171800 & -1.08300800 \\
\hline $\mathrm{H}$ & 3.24201900 & 2.44809800 & 1.79105000 & $\mathrm{O}$ & -1.44022400 & -2.71737700 & -1.39221000 \\
\hline $\mathrm{C}$ & 2.53835600 & 2.55994800 & -1.61577400 & $\mathrm{C}$ & 0.04927000 & -0.84458600 & -1.43358000 \\
\hline
\end{tabular}




\begin{tabular}{|c|c|c|c|c|c|c|c|}
\hline $\mathrm{H}$ & 1.75776400 & 0.75142100 & -0.49786200 & $\mathrm{C}$ & 4.89618100 & -0.73110800 & -0.11025100 \\
\hline $\mathrm{H}$ & -0.06182200 & 0.24146400 & -1.44478400 & $\mathrm{H}$ & 4.24909100 & 1.25001100 & -0.66286200 \\
\hline $\mathrm{H}$ & 0.33506500 & -1.18095700 & -2.43727300 & $\mathrm{H}$ & 1.83274900 & 0.73491200 & -0.56799000 \\
\hline $\mathrm{C}$ & 1.09211200 & 3.18379400 & -0.12550900 & $\mathrm{H}$ & 2.79713700 & -3.32949800 & 0.56294000 \\
\hline $\mathrm{C}$ & 2.25538700 & 3.39652800 & 0.88377800 & $\mathrm{H}$ & 5.23633300 & -2.78550600 & 0.47397200 \\
\hline $\mathrm{H}$ & 3.11114100 & 2.77367300 & 0.59451200 & $\mathrm{H}$ & 5.95573400 & -0.48331700 & -0.14642000 \\
\hline $\mathrm{H}$ & 2.59506800 & 4.44248600 & 0.94937700 & $\mathrm{C}$ & 0.72473100 & -1.76359100 & 0.03456900 \\
\hline $\mathrm{H}$ & 1.93284600 & 3.07273700 & 1.88138300 & $\mathrm{O}$ & 0.41277100 & -2.83099600 & 0.58385700 \\
\hline $\mathrm{C}$ & 1.56907000 & 3.63715000 & -1.53418800 & $\mathrm{C}$ & -0.28059300 & -0.85732800 & -0.61606200 \\
\hline $\mathrm{H}$ & 2.41112300 & 3.01092800 & -1.85436500 & $\mathrm{H}$ & -1.35979400 & -3.04618800 & -0.12210200 \\
\hline $\mathrm{H}$ & 0.75569700 & 3.49192000 & -2.25640800 & $\mathrm{H}$ & -0.07618900 & -0.89304900 & -1.69900300 \\
\hline $\mathrm{H}$ & 1.88596000 & 4.69161900 & -1.57424300 & $\mathrm{H}$ & -0.08906500 & 0.21549000 & -0.35941300 \\
\hline $\mathrm{C}$ & -0.09861000 & 4.08402600 & 0.30344900 & $\mathrm{C}$ & 0.29354200 & 3.14669300 & 0.09244300 \\
\hline $\mathrm{H}$ & -0.92949100 & 3.95295400 & -0.40186100 & $\mathrm{C}$ & -1.12410200 & 3.79600700 & 0.05362800 \\
\hline $\mathrm{H}$ & -0.44962500 & 3.77310400 & 1.29608600 & $\mathrm{H}$ & -1.84964600 & 3.11289100 & 0.51493000 \\
\hline $\mathrm{H}$ & 0.15433200 & 5.15509200 & 0.34467000 & $\mathrm{H}$ & -1.18729100 & 4.76716100 & 0.57169500 \\
\hline $\mathrm{O}$ & 0.70294100 & 1.86712500 & -0.14423000 & $\mathrm{H}$ & -1.42410500 & 3.93902600 & -0.99240000 \\
\hline $\mathrm{C}$ & 3.09492900 & -2.84507800 & 0.06410700 & $\mathrm{C}$ & 0.68763100 & 2.94371200 & 1.58752100 \\
\hline $\mathrm{H}$ & 3.89151500 & -2.11002300 & -0.10576500 & $\mathrm{H}$ & -0.01369800 & 2.24194000 & 2.05806300 \\
\hline $\mathrm{H}$ & 3.49954200 & -3.83236900 & -0.20470900 & $\mathrm{H}$ & 1.68929600 & 2.49841100 & 1.64605100 \\
\hline $\mathrm{C}$ & 1.90979800 & -2.49286600 & -0.84747900 & $\mathrm{H}$ & 0.68994700 & 3.87428800 & 2.17880700 \\
\hline $\mathrm{H}$ & 2.24524600 & -2.44555700 & -1.89209400 & $\mathrm{C}$ & 1.29885800 & 4.16195200 & -0.53522500 \\
\hline $\mathrm{H}$ & 1.13731600 & -3.27202800 & -0.79122300 & $\mathrm{H}$ & 2.31455200 & 3.74631100 & -0.48928200 \\
\hline $\mathrm{C}$ & 2.69379100 & -2.81775200 & 1.54665200 & $\mathrm{H}$ & 1.04856200 & 4.30471200 & -1.59421300 \\
\hline $\mathrm{H}$ & 3.56656500 & -3.02823300 & 2.18293200 & $\mathrm{H}$ & 1.30821100 & 5.14770700 & -0.04170500 \\
\hline $\mathrm{H}$ & 1.96407200 & -3.62055100 & 1.74060000 & $\mathrm{O}$ & 0.30615700 & 1.97775300 & -0.60522400 \\
\hline $\mathrm{C}$ & 2.06950300 & -1.46422900 & 1.91638200 & $\mathrm{C}$ & -4.12551300 & -0.49077200 & -1.04971400 \\
\hline $\mathrm{H}$ & 2.82196400 & -0.67490100 & 1.79972400 & $\mathrm{H}$ & -4.42976300 & -1.52451000 & -1.26169800 \\
\hline $\mathrm{H}$ & 1.75768800 & -1.46273300 & 2.97089500 & $\mathrm{H}$ & -4.69075900 & 0.15709600 & -1.73486700 \\
\hline $\mathrm{C}$ & 1.27823800 & -1.13450000 & -0.48448800 & $\mathrm{C}$ & -2.61993300 & -0.35096100 & -1.32099600 \\
\hline $\mathrm{C}$ & 0.87354400 & -1.12559800 & 1.01385300 & $\mathrm{H}$ & -2.39414600 & -0.67507700 & -2.34564000 \\
\hline $\mathrm{H}$ & 0.06499100 & -1.85042200 & 1.19457400 & $\mathrm{H}$ & -2.29692500 & 0.69530000 & -1.23576700 \\
\hline $\mathrm{H}$ & 0.49884300 & -0.12499000 & 1.25203100 & $\mathrm{C}$ & -4.47348800 & -0.15366700 & 0.40832700 \\
\hline \multirow[t]{2}{*}{$\mathrm{O}$} & 2.24011000 & -0.13334800 & -0.72377100 & $\mathrm{H}$ & -5.54727900 & -0.30765600 & 0.59105200 \\
\hline & & & & $\mathrm{H}$ & -4.27135500 & 0.91252300 & 0.59224400 \\
\hline ab4 & & & & $\mathrm{C}$ & -3.63542600 & -0.99998300 & 1.37830800 \\
\hline $\mathrm{C}$ & 3.93807100 & 0.23962100 & -0.40593500 & $\mathrm{H}$ & -3.89825300 & -2.05900600 & 1.25425900 \\
\hline $\mathrm{C}$ & 2.57190400 & -0.06067700 & -0.37157900 & $\mathrm{H}$ & -3.86520700 & -0.72871200 & 2.41860500 \\
\hline $\mathrm{C}$ & 2.17052200 & -1.36448700 & -0.02444600 & $\mathrm{C}$ & -1.75968600 & -1.18410800 & -0.35431100 \\
\hline $\mathrm{C}$ & 3.13791500 & -2.33598100 & 0.28686700 & $\mathrm{C}$ & -2.13442200 & -0.82801400 & 1.10685900 \\
\hline $\mathrm{C}$ & 4.49327800 & -2.02584800 & 0.23924700 & $\mathrm{H}$ & -1.83776900 & 0.21313800 & 1.29440200 \\
\hline
\end{tabular}




\begin{tabular}{|c|c|c|c|c|c|c|c|}
\hline $\mathrm{H}$ & -1.54946900 & -1.45726000 & 1.78903200 & $\mathrm{H}$ & -2.32929400 & 0.37135100 & -1.65026600 \\
\hline \multirow[t]{2}{*}{$\mathrm{O}$} & -2.05614100 & -2.56799500 & -0.61465700 & $\mathrm{C}$ & -4.56002000 & -0.41076100 & -0.06117500 \\
\hline & & & & $\mathrm{H}$ & -5.61632500 & -0.69624200 & 0.05587000 \\
\hline \multicolumn{4}{|l|}{ TS13 } & $\mathrm{H}$ & -4.53844800 & 0.68720400 & -0.14346800 \\
\hline \multicolumn{4}{|c|}{ imaginary frequency $=-1052.95 \mathrm{~cm}^{-1}$} & $\mathrm{C}$ & -3.74441200 & -0.83911100 & 1.16852400 \\
\hline $\mathrm{C}$ & 4.05409800 & 0.18081200 & -0.94205500 & $\mathrm{H}$ & -3.83868900 & -1.92435100 & 1.30396500 \\
\hline $\mathrm{C}$ & 2.69109100 & 0.00252800 & -0.69173500 & $\mathrm{H}$ & -4.14456600 & -0.36205100 & 2.07521500 \\
\hline $\mathrm{C}$ & 2.25413100 & -1.10973000 & 0.04847300 & $\mathrm{C}$ & -1.65189300 & -1.13024500 & -0.28059800 \\
\hline $\mathrm{C}$ & 3.20363500 & -2.01755500 & 0.54112600 & $\mathrm{C}$ & -2.25926300 & -0.49061900 & 0.99477300 \\
\hline $\mathrm{C}$ & 4.56151600 & -1.84042200 & 0.28170500 & $\mathrm{H}$ & -2.14044600 & 0.59825700 & 0.92556300 \\
\hline $\mathrm{C}$ & 4.99159000 & -0.73934300 & -0.46567000 & $\mathrm{H}$ & -1.67925400 & -0.82554200 & 1.86245800 \\
\hline $\mathrm{H}$ & 4.38555400 & 1.05011900 & -1.50661900 & $\mathrm{O}$ & -1.77133700 & -2.56726600 & -0.17967300 \\
\hline $\mathrm{H}$ & 1.96370700 & 0.74382000 & -1.02174600 & & & & \\
\hline $\mathrm{H}$ & 2.84441700 & -2.85606000 & 1.13023700 & ab5 & & & \\
\hline $\mathrm{H}$ & 5.28682900 & -2.55747400 & 0.66236900 & $\mathrm{C}$ & 3.79075700 & -1.09218800 & -1.47898200 \\
\hline $\mathrm{H}$ & 6.05163700 & -0.59582500 & -0.66736900 & $\mathrm{C}$ & 2.46339600 & -0.83709900 & -1.13017300 \\
\hline $\mathrm{C}$ & 0.79371600 & -1.36328500 & 0.34490900 & $\mathrm{C}$ & 1.93390100 & -1.30240100 & 0.08612400 \\
\hline $\mathrm{O}$ & 0.51775400 & -2.13933500 & 1.29181400 & $\mathrm{C}$ & 2.79063600 & -2.00144000 & 0.95183000 \\
\hline $\mathrm{C}$ & -0.19477400 & -0.69158600 & -0.47779200 & $\mathrm{C}$ & 4.11484000 & -2.26502000 & 0.60206100 \\
\hline $\mathrm{H}$ & -1.09170300 & -2.79012700 & 0.49097000 & $\mathrm{C}$ & 4.62356500 & -1.81563000 & -0.61974300 \\
\hline $\mathrm{H}$ & 0.10507400 & -0.72341700 & -1.53408500 & $\mathrm{H}$ & 4.18076500 & -0.71300000 & -2.42226000 \\
\hline $\mathrm{H}$ & -0.10371400 & 0.62695700 & -0.39047100 & $\mathrm{H}$ & 1.84382700 & -0.23156600 & -1.78441300 \\
\hline $\mathrm{C}$ & 0.07844800 & 2.93052900 & 0.17855000 & $\mathrm{H}$ & 2.37667900 & -2.31979400 & 1.90345200 \\
\hline $\mathrm{C}$ & -1.30872900 & 3.61169100 & 0.32047500 & $\mathrm{H}$ & 4.75598400 & -2.81892100 & 1.28657400 \\
\hline $\mathrm{H}$ & -2.04290900 & 2.89492900 & 0.70790900 & $\mathrm{H}$ & 5.65852500 & -2.01305900 & -0.89335800 \\
\hline $\mathrm{H}$ & -1.29388100 & 4.48336500 & 0.99283200 & $\mathrm{C}$ & 0.50200500 & -1.09281900 & 0.53368700 \\
\hline $\mathrm{H}$ & -1.65448500 & 3.93955700 & -0.66751100 & $\mathrm{O}$ & 0.28620900 & -1.18043700 & 1.80359900 \\
\hline $\mathrm{C}$ & 0.54471600 & 2.44210900 & 1.57290600 & $\mathrm{C}$ & -0.48218100 & -0.87170300 & -0.41805600 \\
\hline $\mathrm{H}$ & -0.15827900 & 1.70394600 & 1.97632200 & $\mathrm{H}$ & -1.34984400 & -1.62983800 & 1.63640800 \\
\hline $\mathrm{H}$ & 1.52568100 & 1.95893500 & 1.49375900 & $\mathrm{H}$ & -0.23763400 & -0.96632000 & -1.47361500 \\
\hline $\mathrm{H}$ & 0.62484500 & 3.26583100 & 2.29780500 & $\mathrm{H}$ & 0.17748500 & 1.22753300 & -0.60375900 \\
\hline $\mathrm{C}$ & 1.08973100 & 3.98539500 & -0.33923900 & $\mathrm{C}$ & 0.93636100 & 2.96132800 & 0.03983900 \\
\hline $\mathrm{H}$ & 2.08631000 & 3.53438300 & -0.42110800 & $\mathrm{C}$ & -0.27730600 & 3.66046400 & 0.68299400 \\
\hline $\mathrm{H}$ & 0.78979800 & 4.31730800 & -1.34077500 & $\mathrm{H}$ & -0.91834500 & 2.92872000 & 1.18498300 \\
\hline $\mathrm{H}$ & 1.16208000 & 4.86794700 & 0.31475700 & $\mathrm{H}$ & 0.03357700 & 4.40896100 & 1.42461500 \\
\hline $\mathrm{O}$ & 0.01104600 & 1.90047500 & -0.75004100 & $\mathrm{H}$ & -0.87409700 & 4.16089100 & -0.08852700 \\
\hline $\mathrm{C}$ & -3.97969100 & -1.02532800 & -1.34475800 & $\mathrm{C}$ & 1.74896300 & 2.20999600 & 1.10747900 \\
\hline $\mathrm{H}$ & -4.10411100 & -2.11544900 & -1.30844600 & $\mathrm{H}$ & 1.15186600 & 1.42734800 & 1.58995800 \\
\hline $\mathrm{H}$ & -4.53702100 & -0.66688500 & -2.22288300 & $\mathrm{H}$ & 2.61821200 & 1.72539300 & 0.65092200 \\
\hline $\mathrm{C}$ & -2.48512300 & -0.70588800 & -1.50234500 & $\mathrm{H}$ & 2.10409100 & 2.89963400 & 1.88519800 \\
\hline $\mathrm{H}$ & -2.08510800 & -1.21662000 & -2.38851200 & $\mathrm{C}$ & 1.81824600 & 3.99670000 & -0.67332100 \\
\hline
\end{tabular}




\begin{tabular}{|c|c|c|c|c|c|c|c|}
\hline $\mathrm{H}$ & 2.67656900 & 3.50057400 & -1.13954400 & $\mathrm{C}$ & -0.25241200 & 2.73778600 & 1.26515700 \\
\hline $\mathrm{H}$ & 1.24692000 & 4.49985800 & -1.46251300 & $\mathrm{H}$ & 0.11557700 & 1.71255600 & 1.14518400 \\
\hline $\mathrm{H}$ & 2.18881200 & 4.75587700 & 0.02687800 & $\mathrm{H}$ & 0.61442000 & 3.40247200 & 1.38356000 \\
\hline $\mathrm{O}$ & 0.49466900 & 2.07869200 & -0.99225700 & $\mathrm{H}$ & -0.85144200 & 2.78339900 & 2.18340400 \\
\hline $\mathrm{C}$ & -4.28629200 & -1.56047600 & -0.89958000 & $\mathrm{C}$ & -0.28212900 & 3.09289300 & -1.23819700 \\
\hline $\mathrm{H}$ & -4.45203500 & -2.22871600 & -0.04531000 & $\mathrm{H}$ & 0.15157900 & 2.09767600 & -1.39488700 \\
\hline $\mathrm{H}$ & -4.83705200 & -1.98289100 & -1.75366000 & $\mathrm{H}$ & -0.91888000 & 3.33393500 & -2.09867400 \\
\hline $\mathrm{C}$ & -2.78074300 & -1.52542800 & -1.20106900 & $\mathrm{H}$ & 0.54141000 & 3.81955300 & -1.20607500 \\
\hline $\mathrm{H}$ & -2.40524500 & -2.53876900 & -1.39046700 & $\mathrm{C}$ & -1.69863900 & 4.54620900 & 0.26417600 \\
\hline $\mathrm{H}$ & -2.59904400 & -0.93435200 & -2.11203400 & $\mathrm{H}$ & -2.31723400 & 4.83123100 & -0.59501100 \\
\hline $\mathrm{C}$ & -4.82488100 & -0.16191500 & -0.55883100 & $\mathrm{H}$ & -2.33407500 & 4.56192500 & 1.15784800 \\
\hline $\mathrm{H}$ & -5.89481300 & -0.21428400 & -0.30653400 & $\mathrm{H}$ & -0.90423500 & 5.29306200 & 0.38726800 \\
\hline $\mathrm{H}$ & -4.74489400 & 0.48400700 & -1.44834300 & $\mathrm{O}$ & -2.24945400 & 2.28117200 & -0.04687700 \\
\hline $\mathrm{C}$ & -4.02814800 & 0.46521800 & 0.59569700 & $\mathrm{C}$ & -3.66381600 & -1.22218700 & 0.92778300 \\
\hline $\mathrm{H}$ & -4.17894200 & -0.13422900 & 1.50217700 & $\mathrm{H}$ & -3.95960500 & -0.44254300 & 0.21503600 \\
\hline $\mathrm{H}$ & -4.39891600 & 1.47926900 & 0.80760200 & $\mathrm{H}$ & -4.28073300 & -1.08028300 & 1.82730800 \\
\hline $\mathrm{C}$ & -1.95865300 & -0.91739300 & -0.04307500 & $\mathrm{C}$ & -2.17866400 & -1.02906600 & 1.27382100 \\
\hline $\mathrm{C}$ & -2.52399600 & 0.49437500 & 0.28437700 & $\mathrm{H}$ & -2.01206500 & -0.01410200 & 1.65096000 \\
\hline $\mathrm{H}$ & -2.32287100 & 1.17410600 & -0.55656100 & $\mathrm{H}$ & -1.89109600 & -1.72794000 & 2.07474000 \\
\hline $\mathrm{H}$ & -1.96631900 & 0.87700300 & 1.14799100 & $\mathrm{C}$ & -3.93224700 & -2.60402000 & 0.31158800 \\
\hline \multirow[t]{2}{*}{$\mathrm{O}$} & -2.18038900 & -1.75961000 & 1.09698300 & $\mathrm{H}$ & -4.99187100 & -2.69855200 & 0.03105700 \\
\hline & & & & $\mathrm{H}$ & -3.73887600 & -3.38317900 & 1.06726500 \\
\hline ab6 & & & & $\mathrm{C}$ & -3.02819700 & -2.84703100 & -0.90658500 \\
\hline $\mathrm{C}$ & 4.48277100 & -0.84215200 & 1.49086300 & $\mathrm{H}$ & -3.28212700 & -2.12553300 & -1.69315700 \\
\hline $\mathrm{C}$ & 3.11328100 & -0.87497100 & 1.22890400 & $\mathrm{H}$ & -3.20364200 & -3.85254200 & -1.31791800 \\
\hline $\mathrm{C}$ & 2.61271300 & -0.64127800 & -0.06430200 & $\mathrm{C}$ & -1.24708500 & -1.26265300 & 0.06452300 \\
\hline $\mathrm{C}$ & 3.54104700 & -0.35601700 & -1.07989600 & $\mathrm{C}$ & -1.54677200 & -2.66437200 & -0.54365400 \\
\hline $\mathrm{C}$ & 4.91158700 & -0.32446000 & -0.82233400 & $\mathrm{H}$ & -1.24189400 & -3.43645900 & 0.17986900 \\
\hline $\mathrm{C}$ & 5.39437000 & -0.56993200 & 0.46545300 & $\mathrm{H}$ & -0.91117900 & -2.78669200 & -1.42863100 \\
\hline $\mathrm{H}$ & 4.84105800 & -1.02221600 & 2.50335900 & $\mathrm{O}$ & -1.58433300 & -0.27290700 & -0.93041100 \\
\hline
\end{tabular}

\section{TS14}

imaginary frequency $=-74.35 \mathrm{~cm}^{-1}$

$\mathrm{H}$

$\begin{array}{lll}3.13900800 & -0.15822900 & -2.06806600\end{array}$

$\begin{array}{lll}5.60817000 & -0.10404000 & -1.63015300\end{array}$

$\begin{array}{lll}6.46284900 & -0.54238400 & 0.67113700\end{array}$

$\mathrm{C}$

$-3.40274500 \quad-2.52540000$

0.47365400

$\begin{array}{lll}1.15065300 & -0.67122200 & -0.44013500\end{array}$

C

$\begin{array}{lll}0.86687700 & -0.23306200 & -1.63677000\end{array}$

$-2.64655500-1.35558700$

0.36925500

C

$-3.26211300-0.14823100$

0.00346900

$0.21372600 \quad-1.14694900 \quad 0.44877600$

$-4.63410500 \quad-0.13816900$

$-0.28034400$

$\begin{array}{lll}-0.71105300 & -0.17718800 & -1.44821500\end{array}$

$-5.39192300-1.30177900$

$-0.15679000$

$\begin{array}{lll}0.50472700 & -1.56812600 & 1.40644200\end{array}$

C

C

$-1.95172700$

1.38366400

$-0.34076200$

$-4.77728100 \quad-2.49934400$

0.22435800

$\begin{array}{lll}-1.11757100 & 3.14030400 & 0.05484100\end{array}$

$-2.91603300-3.46038000$

0.74247300

$\begin{array}{lll}-1.56846400 & -1.36821100 & 0.51813900\end{array}$ 


\begin{tabular}{|c|c|c|c|}
\hline $\mathrm{H}$ & -5.08461600 & 0.79652100 & -0.60253800 \\
\hline $\mathrm{H}$ & -6.46035400 & -1.28100000 & -0.36328300 \\
\hline $\mathrm{H}$ & -5.36607300 & -3.41009100 & 0.31394700 \\
\hline $\mathrm{C}$ & -2.44638500 & 1.11699400 & -0.17382100 \\
\hline $\mathrm{O}$ & -2.78593500 & 1.91345600 & -1.06647200 \\
\hline $\mathrm{C}$ & -1.32499700 & 1.26323700 & 0.72706900 \\
\hline $\mathrm{H}$ & 0.51512400 & -0.16577000 & -1.06485300 \\
\hline $\mathrm{H}$ & -1.35664700 & 0.64455300 & 1.62078500 \\
\hline $\mathrm{H}$ & 2.45800500 & -0.47682300 & -0.57532100 \\
\hline $\mathrm{C}$ & 3.47448300 & -2.13818200 & -0.19300000 \\
\hline $\mathrm{C}$ & 3.04866400 & -2.16343500 & 1.29166600 \\
\hline $\mathrm{H}$ & 2.04796500 & -1.72643400 & 1.37150200 \\
\hline $\mathrm{H}$ & 3.03725400 & -3.18192100 & 1.70757400 \\
\hline $\mathrm{H}$ & 3.73957500 & -1.55382400 & 1.88786400 \\
\hline $\mathrm{C}$ & 2.51136300 & -3.02490300 & -1.01424000 \\
\hline $\mathrm{H}$ & 1.50073900 & -2.61193100 & -0.92620100 \\
\hline $\mathrm{H}$ & 2.79967700 & -3.00765600 & -2.07293400 \\
\hline $\mathrm{H}$ & 2.50237800 & -4.07022600 & -0.67221700 \\
\hline $\mathrm{C}$ & 4.91936100 & -2.63921300 & -0.34470800 \\
\hline $\mathrm{H}$ & 5.21956900 & -2.60525500 & -1.39925100 \\
\hline $\mathrm{H}$ & 5.60039200 & -1.98978700 & 0.21864300 \\
\hline $\mathrm{H}$ & 5.03709000 & -3.66929200 & 0.01938100 \\
\hline $\mathrm{O}$ & 3.44571300 & -0.81882800 & -0.68556700 \\
\hline $\mathrm{C}$ & 2.32707800 & 2.23968500 & 1.15265500 \\
\hline $\mathrm{H}$ & 2.70656000 & 1.27702600 & 0.80276100 \\
\hline $\mathrm{H}$ & 2.93164700 & 2.55205100 & 2.01758900 \\
\hline $\mathrm{C}$ & 0.86575600 & 2.06640200 & 1.59575300 \\
\hline $\mathrm{H}$ & 0.77461900 & 1.22396800 & 2.28868300 \\
\hline $\mathrm{H}$ & 0.56073400 & 2.97172100 & 2.15421900 \\
\hline $\mathrm{C}$ & 2.45485100 & 3.26864600 & 0.02048800 \\
\hline $\mathrm{H}$ & 3.50176500 & 3.33764700 & -0.30618600 \\
\hline $\mathrm{H}$ & 2.17312500 & 4.27055300 & 0.38817400 \\
\hline $\mathrm{C}$ & 1.55481600 & 2.88485100 & -1.16119200 \\
\hline $\mathrm{H}$ & 1.86995700 & 1.90770100 & -1.53944200 \\
\hline $\mathrm{H}$ & 1.64970800 & 3.62154900 & -1.97254900 \\
\hline $\mathrm{C}$ & -0.14058600 & 1.90433000 & 0.47339700 \\
\hline $\mathrm{C}$ & 0.08070100 & 2.77928700 & -0.73994100 \\
\hline $\mathrm{H}$ & -0.29112500 & 3.78665700 & -0.48081400 \\
\hline $\mathrm{H}$ & -0.55301900 & 2.44538600 & -1.56463600 \\
\hline $\mathrm{O}$ & 1.05374000 & -0.06861000 & -0.26269300 \\
\hline
\end{tabular}

\section{TS14a}

imaginary frequency $=-1720.09 \mathrm{~cm}^{-1}$

$$
\mathrm{C}
$$

C

$4.09284400-1.45850900$

0.07047700

C

$2.73629600-1.14266700$

0.15884200

C

$\begin{array}{lll}2.29845400 & 0.17944100 & -0.01015700\end{array}$

C

C

$3.25121000 \quad 1.17873500 \quad-0.25899400$

$\mathrm{H}$

$\mathrm{H}$

$\mathrm{H}$

$\mathrm{H}$

$\mathrm{H}$

C

O

C

C

$\mathrm{H}$

$\mathrm{H}$

$\mathrm{H}$

C

$\mathrm{H}$

$\mathrm{H}$

C

$\mathrm{H}$

$\mathrm{H}$

C

$\mathrm{H}$

$\mathrm{H}$

C

$\mathrm{H}$

$\mathrm{H}$

C

C

$\mathrm{H}$

$\mathrm{H}$

$\mathrm{O}$

$\begin{array}{lll}4.60445300 & 0.86389300 & -0.35749600\end{array}$

$\begin{array}{lll}5.02972700 & -0.45754900 & -0.19406300\end{array}$

$\begin{array}{lll}4.41838900 & -2.48584600 & 0.21270200\end{array}$

$\begin{array}{lll}2.02238300 & -1.92826800 & 0.38580500\end{array}$

$\begin{array}{lll}2.90063000 & 2.19973300 & -0.36854500\end{array}$

$\begin{array}{lll}5.32998800 & 1.64801400 & -0.55845600\end{array}$

$\begin{array}{lll}6.08571200 & -0.70488600 & -0.26692800\end{array}$

$\begin{array}{lll}0.84886300 & 0.60569900 & 0.08214400\end{array}$

$\begin{array}{lll}0.59675400 & 1.80546100 & 0.23303000\end{array}$

$\begin{array}{lll}-0.16363700 & -0.44973800 & 0.00167600\end{array}$

$\begin{array}{lll}-2.15966000 & -1.76315100 & 1.64513600\end{array}$

$\begin{array}{lll}-0.64355400 & -0.87252100 & 1.29347900\end{array}$

$\begin{array}{lll}0.14361100 & -1.35270300 & -0.52470900\end{array}$

$-3.93480700 \quad-1.14539100 \quad-0.61041700$

$\begin{array}{lll}-4.13121600 & -1.60335200 & 0.36954300\end{array}$

$\begin{array}{lll}-4.45080300 & -1.76515300 & -1.35381400\end{array}$

$-2.42560300 \quad-1.16433300 \quad-0.89224400$

$\begin{array}{lll}-2.01327300 & -2.17864100 & -0.81691200\end{array}$

$\begin{array}{lll}-2.24689600 & -0.84629200 & -1.93110200\end{array}$

$\begin{array}{llll}-4.48323000 & 0.28799400 & -0.61553600\end{array}$

$\begin{array}{lll}-5.55306900 & 0.28271100 & -0.37325900\end{array}$

$\begin{array}{lll}-4.39301600 & 0.70717000 & -1.62876500\end{array}$

$\begin{array}{lll}-3.71366000 & 1.16897900 & 0.37756500\end{array}$

$\begin{array}{lll}-3.88306500 & 0.80240100 & 1.39793200\end{array}$

$\begin{array}{lll}-4.08745100 & 2.19928000 & 0.34241900\end{array}$

$\begin{array}{lll}-1.60982900 & -0.20732000 & -0.04090600\end{array}$

$\begin{array}{lll}-2.20172200 & 1.17640500 & 0.10810900\end{array}$

$\begin{array}{lll}-1.98171900 & 1.69427200 & -0.83808800\end{array}$

$\begin{array}{lll}-1.65649100 & 1.73197400 & 0.86974900\end{array}$

$\begin{array}{lll}-1.80633600 & -0.85278400 & 1.67069700\end{array}$

\section{TS14b}

imaginary frequency $=-1574.25 \mathrm{~cm}^{-1}$

$\begin{array}{llll}\mathrm{C} & 4.07367400 & 1.47614700 & 0.44524000 \\ \mathrm{C} & 2.70983800 & 1.22480800 & 0.29714600\end{array}$




\begin{tabular}{|c|c|c|c|c|c|c|c|}
\hline $\mathrm{C}$ & 2.25256800 & -0.06190000 & -0.02642300 & $\mathrm{H}$ & -0.98879200 & -1.06599100 & 0.15507100 \\
\hline $\mathrm{C}$ & 3.19131300 & -1.09353000 & -0.18633800 & $\mathrm{H}$ & -5.23969500 & -0.28939800 & -0.15375100 \\
\hline $\mathrm{C}$ & 4.55351200 & -0.84269500 & -0.04122800 & $\mathrm{H}$ & -5.69555500 & -2.74758500 & -0.11757700 \\
\hline $\mathrm{C}$ & 4.99907900 & 0.44431800 & 0.27302000 & $\mathrm{H}$ & -3.79589600 & -4.34492600 & 0.06586100 \\
\hline $\mathrm{H}$ & 4.41368200 & 2.47624600 & 0.70087500 & $\mathrm{C}$ & -2.88015200 & 0.96214800 & 0.04454000 \\
\hline $\mathrm{H}$ & 1.99996700 & 2.03037700 & 0.45583700 & $\mathrm{O}$ & -3.60280200 & 1.69424300 & -0.63358000 \\
\hline $\mathrm{H}$ & 2.82428900 & -2.08630800 & -0.42352600 & $\mathrm{C}$ & -1.77534600 & 1.46355400 & 0.89614600 \\
\hline $\mathrm{H}$ & 5.26971200 & -1.64964600 & -0.17237300 & $\mathrm{H}$ & 0.63387200 & -1.03990600 & -1.38666800 \\
\hline $\mathrm{H}$ & 6.06180300 & 0.64121700 & 0.38831600 & $\mathrm{H}$ & -1.66091400 & 0.94829600 & 1.84948800 \\
\hline $\mathrm{C}$ & 0.80162100 & -0.39414500 & -0.18857100 & $\mathrm{H}$ & 2.24165800 & -0.48460400 & -0.55217900 \\
\hline $\mathrm{O}$ & 0.47303500 & -1.62366000 & -0.19995400 & $\mathrm{C}$ & 3.89796700 & -1.46860100 & -0.01558000 \\
\hline $\mathrm{C}$ & -0.17647200 & 0.62560500 & -0.42870200 & $\mathrm{C}$ & 3.40333200 & -1.68875900 & 1.43291100 \\
\hline $\mathrm{H}$ & -0.99191600 & -1.50676900 & -0.93219800 & $\mathrm{H}$ & 2.31168100 & -1.77213600 & 1.41561100 \\
\hline $\mathrm{H}$ & -0.75265400 & -0.10656900 & -1.66063700 & $\mathrm{H}$ & 3.82971100 & -2.59296400 & 1.89324300 \\
\hline $\mathrm{H}$ & 0.09379900 & 1.67504700 & -0.38814100 & $\mathrm{H}$ & 3.67283500 & -0.82427400 & 2.05305100 \\
\hline $\mathrm{C}$ & -4.07301000 & 0.81993300 & -0.61756400 & $\mathrm{C}$ & 3.52190900 & -2.70301800 & -0.86905400 \\
\hline $\mathrm{H}$ & -4.18997900 & 0.07204100 & -1.41218400 & $\mathrm{H}$ & 2.42944200 & -2.78234900 & -0.88497700 \\
\hline $\mathrm{H}$ & -4.74385400 & 1.65206900 & -0.86383300 & $\mathrm{H}$ & 3.87383200 & -2.56448400 & -1.89962600 \\
\hline $\mathrm{C}$ & -2.61969000 & 1.31966700 & -0.61638500 & $\mathrm{H}$ & 3.94902700 & -3.63859500 & -0.47794700 \\
\hline $\mathrm{H}$ & -2.33951300 & 1.71130400 & -1.60171400 & $\mathrm{C}$ & 5.42042300 & -1.25320600 & -0.03149200 \\
\hline $\mathrm{H}$ & -2.50821800 & 2.15010900 & 0.09549400 & $\mathrm{H}$ & 5.76310000 & -1.08341100 & -1.05978400 \\
\hline $\mathrm{C}$ & -4.45954000 & 0.19566200 & 0.73136300 & $\mathrm{H}$ & 5.67690200 & -0.36396800 & 0.55748800 \\
\hline $\mathrm{H}$ & -5.48382400 & -0.19491500 & 0.68738500 & $\mathrm{H}$ & 5.96499400 & -2.11466100 & 0.38074800 \\
\hline $\mathrm{H}$ & -4.45362900 & 0.97391600 & 1.50937500 & $\mathrm{O}$ & 3.30749900 & -0.31675300 & -0.55942300 \\
\hline $\mathrm{C}$ & -3.47935900 & -0.91992800 & 1.12246100 & $\mathrm{C}$ & 1.63681900 & 2.30927800 & 0.90913900 \\
\hline $\mathrm{H}$ & -3.57246900 & -1.75310000 & 0.41288500 & $\mathrm{H}$ & 1.67815300 & 1.21790800 & 0.85578800 \\
\hline $\mathrm{H}$ & -3.72824400 & -1.32086200 & 2.11287400 & $\mathrm{H}$ & 2.44723000 & 2.64627300 & 1.57065400 \\
\hline $\mathrm{C}$ & -1.62307000 & 0.24254800 & -0.19628000 & $\mathrm{C}$ & 0.27463400 & 2.73832300 & 1.49972800 \\
\hline $\mathrm{C}$ & -2.02585300 & -0.41835800 & 1.12491900 & $\mathrm{H}$ & 0.11815800 & 2.27454900 & 2.48164200 \\
\hline $\mathrm{H}$ & -1.90582600 & 0.35219800 & 1.89965500 & $\mathrm{H}$ & 0.27474600 & 3.83152300 & 1.65132700 \\
\hline $\mathrm{H}$ & -1.32659800 & -1.22280700 & 1.36587000 & $\mathrm{C}$ & 1.83722800 & 2.85547300 & -0.51196800 \\
\hline \multirow[t]{2}{*}{$\mathrm{O}$} & -1.64980900 & -0.82663200 & -1.34128300 & $\mathrm{H}$ & 2.75914400 & 2.42559900 & -0.92002500 \\
\hline & & & & $\mathrm{H}$ & 1.95787500 & 3.95251300 & -0.48646900 \\
\hline ab7 & & & & $\mathrm{C}$ & 0.64917800 & 2.47780500 & -1.40946500 \\
\hline $\mathrm{C}$ & -2.29786800 & -2.79155300 & 0.14889800 & $\mathrm{H}$ & 0.61337600 & 1.38093600 & -1.46658300 \\
\hline $\mathrm{C}$ & -2.03688500 & -1.41713200 & 0.16566700 & $\mathrm{H}$ & 0.77951200 & 2.88466700 & -2.42248400 \\
\hline $\mathrm{C}$ & -3.11644200 & -0.52198800 & 0.07391300 & $\mathrm{C}$ & -0.85143000 & 2.38279200 & 0.55771700 \\
\hline $\mathrm{C}$ & -4.42815700 & -1.00507200 & -0.05139900 & $\mathrm{C}$ & -0.69417900 & 2.97699900 & -0.81961100 \\
\hline $\mathrm{C}$ & -4.67569700 & -2.37573800 & -0.03980100 & $\mathrm{H}$ & -0.67508900 & 4.07703600 & -0.74491400 \\
\hline $\mathrm{C}$ & -3.60581500 & -3.27294800 & 0.06363900 & $\mathrm{H}$ & -1.53553200 & 2.70948000 & -1.46215000 \\
\hline $\mathrm{H}$ & -1.45937400 & -3.48286800 & 0.19421100 & $\mathrm{O}$ & 0.84511200 & -0.77333900 & -0.47607400 \\
\hline
\end{tabular}




\section{$\mathrm{H}_{2} \mathrm{O}$}

$\mathrm{O}$

$\mathrm{H}$

$\mathrm{H}$

ab8

C

$\mathrm{H}$

$\mathrm{H}$

C

$\mathrm{H}$

$\mathrm{H}$

C

$\mathrm{H}$

$\mathrm{H}$

C

H

$\mathrm{H}$

C

C

$\mathrm{H}$

$\mathrm{H}$

C

C

C

C

C

C

$\mathrm{H}$

$\mathrm{H}$

$\mathrm{H}$

H

$\mathrm{H}$

C

O

C

H

ab9

C

8

更

H

(1)

(

C

H

(

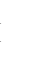

H

C

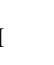

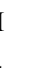

C

C

C

H

$\mathrm{H}$

H

H

C

c

$$
\begin{array}{rrr}
0.00000000 & 0.00000000 & 0.11942500 \\
0.00000000 & 0.76260700 & -0.47770000 \\
0.00000000 & -0.76260700 & -0.47770000
\end{array}
$$

C

$-0.30039800$

\begin{tabular}{|c|c|c|}
\hline-2.83098500 & 1.11466300 & 0.01529400 \\
\hline-2.24308900 & -0.16005000 & -0.00715400 \\
\hline-3.07394300 & -1.29270300 & -0.01465800 \\
\hline-4.45861200 & -1.15552000 & -0.00040000 \\
\hline-5.03471800 & 0.11855000 & 0.02165900 \\
\hline-4.66353500 & 2.24329100 & 0.04763600 \\
\hline-2.21220700 & 2.00612300 & 0.02334900 \\
\hline-2.60543000 & -2.27115900 & -0.03158900 \\
\hline-5.09124200 & -2.03897600 & -0.00618800 \\
\hline-6.11601100 & 0.22718800 & 0.03315600 \\
\hline-0.75587200 & -0.38154200 & -0.02456000 \\
\hline-0.31262500 & -1.52238900 & -0.03768900 \\
\hline 0.14976600 & 0.84748600 & -0.02948900 \\
\hline 2.15070800 & 2.37668700 & -0.82170600 \\
\hline-0.11282600 & 1.47758300 & 0.83104600 \\
\hline-0.11329400 & 1.44556200 & -0.91633000 \\
\hline 3.71596000 & -0.29442900 & -1.23912300 \\
\hline 4.21344600 & 0.68262900 & -1.29345300 \\
\hline 4.02614600 & -0.86031700 & -2.12678200 \\
\hline 2.19220400 & -0.09495800 & -1.26232100 \\
\hline 1.89334500 & 0.46973900 & -2.16036600 \\
\hline 1.68844700 & -1.06552900 & -1.31910100 \\
\hline 4.16014500 & -1.01701000 & 0.04144700 \\
\hline 5.25277100 & -1.12081600 & 0.05773800 \\
\hline 3.74657400 & -2.03654600 & 0.04353700 \\
\hline 3.67810600 & -0.26896300 & 1.29359200 \\
\hline 4.17456000 & 0.70725700 & 1.34930600 \\
\hline 3.95772300 & -0.82349300 & 2.19853900 \\
\hline 1.68221900 & 0.64636400 & -0.01034600 \\
\hline 2.15553200 & -0.05972200 & 1.27246900 \\
\hline 1.64413400 & -1.02540600 & 1.33791300 \\
\hline 1.84380800 & 0.54153000 & 2.13645600 \\
\hline 2.26487400 & 1.96489600 & 0.05033300 \\
\hline
\end{tabular}

$\begin{array}{lll}-4.21923300 & 1.25193400 \quad 0.02953900\end{array}$

$\mathrm{C}$ ab10

$\begin{array}{rrc}4.05143900 & 1.51886400 & 0.33868800 \\ 2.70703000 & 1.23407500 & 0.10061500 \\ 2.28395800 & -0.09221300 & -0.08319500 \\ 3.23449800 & -1.12577400 & -0.02529400 \\ 4.57421300 & -0.84134300 & 0.21897600 \\ 4.98559900 & 0.48285800 & 0.40132400\end{array}$ 


\begin{tabular}{|c|c|c|c|c|c|c|c|}
\hline $\mathrm{H}$ & 4.36913900 & 2.54865100 & 0.47596200 & $\mathrm{C}$ & -0.85041400 & 0.45738400 & -0.01899600 \\
\hline $\mathrm{H}$ & 1.99440700 & 2.05082100 & 0.05087100 & $\mathrm{O}$ & -0.65906300 & 1.79544500 & 0.16676700 \\
\hline $\mathrm{H}$ & 2.89488500 & -2.14517200 & -0.17508600 & $\mathrm{C}$ & 0.16929000 & -0.40239900 & -0.24769500 \\
\hline $\mathrm{H}$ & 5.30009900 & -1.64840500 & 0.26606200 & $\mathrm{H}$ & 0.27903900 & 1.97833900 & -0.07032300 \\
\hline $\mathrm{H}$ & 6.03213200 & 0.70630900 & 0.59066300 & $\mathrm{H}$ & 1.54605100 & 1.27706000 & -1.71874400 \\
\hline $\mathrm{C}$ & 0.85895000 & -0.46270100 & -0.35737900 & $\mathrm{H}$ & -0.06153800 & -1.44437800 & -0.43992800 \\
\hline $\mathrm{O}$ & 0.57209000 & -1.63557700 & -0.59176100 & $\mathrm{C}$ & 3.98653700 & -0.68626600 & -0.99055300 \\
\hline $\mathrm{C}$ & -0.20658300 & 0.62847400 & -0.37012900 & $\mathrm{H}$ & 4.16826500 & 0.27489500 & -1.48832300 \\
\hline $\mathrm{H}$ & -1.23773700 & -1.47481600 & -1.30589600 & $\mathrm{H}$ & 4.54304800 & -1.44593100 & -1.55371300 \\
\hline $\mathrm{H}$ & -0.06273200 & 1.20353500 & -1.29774100 & $\mathrm{C}$ & 2.48521500 & -1.00553600 & -1.04220600 \\
\hline $\mathrm{H}$ & -0.02483300 & 1.32739200 & 0.45503100 & $\mathrm{H}$ & 2.12351600 & -1.01908300 & -2.08090600 \\
\hline $\mathrm{C}$ & -4.10040200 & 0.90725000 & -0.44200700 & $\mathrm{H}$ & 2.30698300 & -2.01458000 & -0.64459000 \\
\hline $\mathrm{H}$ & -4.33033300 & 0.21832400 & -1.26333100 & $\mathrm{C}$ & 4.49326600 & -0.61019600 & 0.45743400 \\
\hline $\mathrm{H}$ & -4.73826600 & 1.79067700 & -0.57435000 & $\mathrm{H}$ & 5.55762700 & -0.34374600 & 0.47224400 \\
\hline $\mathrm{C}$ & -2.62344500 & 1.31752700 & -0.54388500 & $\mathrm{H}$ & 4.41264300 & -1.60452300 & 0.92255100 \\
\hline $\mathrm{H}$ & -2.41751000 & 1.75097300 & -1.53077000 & $\mathrm{C}$ & 3.67808400 & 0.40208400 & 1.27589800 \\
\hline $\mathrm{H}$ & -2.40106700 & 2.09163500 & 0.20500100 & $\mathrm{H}$ & 3.84980200 & 1.41200100 & 0.88393800 \\
\hline $\mathrm{C}$ & -4.40294000 & 0.22288500 & 0.89935700 & $\mathrm{H}$ & 4.01192100 & 0.40454700 & 2.32136000 \\
\hline $\mathrm{H}$ & -5.44943400 & -0.10687700 & 0.92949600 & $\mathrm{C}$ & 1.63285400 & -0.01471800 & -0.22383900 \\
\hline $\mathrm{H}$ & -4.28040900 & 0.95051300 & 1.71669400 & $\mathrm{C}$ & 2.17484200 & 0.08807600 & 1.21902700 \\
\hline $\mathrm{C}$ & -3.46179800 & -0.96742200 & 1.13411100 & $\mathrm{H}$ & 1.97434300 & -0.87126700 & 1.71372700 \\
\hline $\mathrm{H}$ & -3.65732400 & -1.73904000 & 0.37935500 & $\mathrm{H}$ & 1.59424500 & 0.84591300 & 1.75782200 \\
\hline $\mathrm{H}$ & -3.65313000 & -1.42072400 & 2.11518000 & $\mathrm{O}$ & 1.80338900 & 1.33085800 & -0.78179600 \\
\hline $\mathrm{C}$ & -1.67051000 & 0.12726800 & -0.32279500 & & & & \\
\hline $\mathrm{C}$ & -1.98824700 & -0.54562300 & 1.03619300 & \multicolumn{4}{|c|}{ TS15 } \\
\hline $\mathrm{H}$ & -1.75404500 & 0.16195700 & 1.84599600 & \multicolumn{4}{|c|}{ imaginary frequency $=-106.71 \mathrm{~cm}^{-1}$} \\
\hline $\mathrm{H}$ & -1.33525800 & -1.41508800 & 1.17466200 & $\mathrm{C}$ & 2.68272900 & 1.23201300 & -1.20392800 \\
\hline \multirow[t]{2}{*}{$\mathrm{O}$} & -1.90948500 & -0.77385000 & -1.40389900 & $\mathrm{C}$ & 1.62537500 & 0.33134800 & -1.06359700 \\
\hline & & & & $\mathrm{C}$ & 1.71216400 & -0.74218200 & -0.16319300 \\
\hline ab11 & & & & $\mathrm{C}$ & 2.88622300 & -0.87965500 & 0.59494700 \\
\hline $\mathrm{C}$ & -4.03447900 & -1.58998600 & 0.30776200 & $\mathrm{C}$ & 3.94837800 & 0.01014800 & 0.44783200 \\
\hline $\mathrm{C}$ & -2.68436700 & -1.24782800 & 0.29116300 & $\mathrm{C}$ & 3.85169400 & 1.07265700 & -0.45567900 \\
\hline $\mathrm{C}$ & -2.28052600 & 0.07142200 & 0.02430000 & $\mathrm{H}$ & 2.58916700 & 2.06586200 & -1.89663400 \\
\hline $\mathrm{C}$ & -3.27033000 & 1.04295800 & -0.19858400 & $\mathrm{H}$ & 0.71664600 & 0.48402700 & -1.63554600 \\
\hline $\mathrm{C}$ & -4.62165700 & 0.69918600 & -0.17849700 & $\mathrm{H}$ & 2.92842200 & -1.70510700 & 1.29878800 \\
\hline $\mathrm{C}$ & -5.01034800 & -0.61822600 & 0.07020100 & $\mathrm{H}$ & 4.85255700 & -0.11966300 & 1.04040300 \\
\hline $\mathrm{H}$ & -4.32633500 & -2.61528000 & 0.51973900 & $\mathrm{H}$ & 4.67678000 & 1.77297100 & -0.57132800 \\
\hline $\mathrm{H}$ & -1.93824200 & -2.00532200 & 0.51116700 & $\mathrm{C}$ & 0.62697900 & -1.78822500 & 0.03024700 \\
\hline $\mathrm{H}$ & -2.96718100 & 2.06625900 & -0.38921200 & $\mathrm{O}$ & 0.73729400 & -2.54905800 & 1.01239100 \\
\hline $\mathrm{H}$ & -5.37292400 & 1.46403600 & -0.35762700 & $\mathrm{C}$ & -0.49765300 & -1.83737400 & -0.89304900 \\
\hline $\mathrm{H}$ & -6.06367300 & -0.88509600 & 0.08856500 & $\mathrm{H}$ & -0.34305800 & -1.35026900 & -1.85388300 \\
\hline
\end{tabular}




\begin{tabular}{|c|c|c|c|c|c|c|c|}
\hline $\mathrm{H}$ & -0.86001800 & -2.85669400 & -1.02184800 & $\mathrm{H}$ & 2.46713100 & -4.98852300 & -0.87455700 \\
\hline $\mathrm{C}$ & -0.90374300 & 1.99750100 & 1.64447700 & $\mathrm{H}$ & 0.76599500 & -3.71118800 & 0.37727700 \\
\hline $\mathrm{C}$ & -1.14073000 & 0.66824000 & 1.28531700 & $\mathrm{H}$ & 3.04064100 & -0.11419700 & -0.15847700 \\
\hline $\mathrm{C}$ & -1.77125700 & 0.34937700 & 0.07222500 & $\mathrm{H}$ & 4.75557600 & -1.38460400 & -1.40800800 \\
\hline $\mathrm{C}$ & -2.16022000 & 1.40449200 & -0.76392800 & $\mathrm{H}$ & 4.48518700 & -3.83408000 & -1.77858400 \\
\hline $\mathrm{C}$ & -1.91745500 & 2.73404500 & -0.41476100 & $\mathrm{C}$ & 0.72601900 & -1.05446100 & 1.00970700 \\
\hline $\mathrm{C}$ & -1.28983200 & 3.03918300 & 0.79622900 & $\mathrm{C}$ & 0.72669600 & -1.57345000 & 2.47417100 \\
\hline $\mathrm{H}$ & -0.41094000 & 2.22069300 & 2.58963000 & $\mathrm{H}$ & 0.48173500 & -2.64111600 & 2.52665100 \\
\hline $\mathrm{H}$ & -0.81590500 & -0.12778300 & 1.94954600 & $\mathrm{H}$ & 1.70878900 & -1.41279600 & 2.93830300 \\
\hline $\mathrm{H}$ & -2.66900800 & 1.12292200 & -1.68174100 & $\mathrm{H}$ & -0.02967100 & -1.01477000 & 3.03424600 \\
\hline $\mathrm{H}$ & -2.22658000 & 3.53735100 & -1.08324500 & $\mathrm{C}$ & 1.67310200 & 3.37510000 & -0.36387000 \\
\hline $\mathrm{H}$ & -1.10318500 & 4.07452700 & 1.07686900 & $\mathrm{C}$ & 2.24730500 & 3.49395100 & 1.06149800 \\
\hline $\mathrm{C}$ & -2.12978100 & -1.08422200 & -0.39299000 & $\mathrm{H}$ & 3.33398700 & 3.34616200 & 1.03951900 \\
\hline $\mathrm{O}$ & -2.88473500 & -1.14309900 & -1.42784000 & $\mathrm{H}$ & 2.03912500 & 4.47848800 & 1.50167900 \\
\hline $\mathrm{C}$ & -2.54511600 & -1.99191200 & 0.79661500 & $\mathrm{H}$ & 1.81839200 & 2.72420500 & 1.71082700 \\
\hline $\mathrm{H}$ & -1.74644800 & -2.18527700 & 1.51987100 & $\mathrm{C}$ & 2.36114200 & 4.38061800 & -1.29692800 \\
\hline $\mathrm{H}$ & -3.40261000 & -1.54043900 & 1.31634300 & $\mathrm{H}$ & 3.44164600 & 4.19589200 & -1.32246300 \\
\hline $\mathrm{H}$ & -2.87281800 & -2.94845800 & 0.37578900 & $\mathrm{H}$ & 1.97486500 & 4.27167100 & -2.31684200 \\
\hline & & & & $\mathrm{H}$ & 2.18995400 & 5.41286800 & -0.96673200 \\
\hline aa1 & & & & $\mathrm{C}$ & 0.14942700 & 3.60275200 & -0.35999200 \\
\hline $\mathrm{C}$ & -5.13298100 & 0.74511700 & -0.37164800 & $\mathrm{H}$ & -0.23539100 & 3.53489400 & -1.38461100 \\
\hline $\mathrm{C}$ & -3.83541500 & 0.78021300 & 0.13877000 & $\mathrm{H}$ & -0.36538700 & 2.84240000 & 0.23960100 \\
\hline $\mathrm{C}$ & -2.99199700 & -0.34121900 & 0.06631900 & $\mathrm{H}$ & -0.10832800 & 4.59325000 & 0.04023700 \\
\hline $\mathrm{C}$ & -3.50972900 & -1.51038500 & -0.51862400 & $\mathrm{O}$ & 1.97300800 & 2.09164900 & -0.90555500 \\
\hline $\mathrm{C}$ & -4.80665100 & -1.54946100 & -1.02992900 & $\mathrm{H}$ & 1.56997600 & 1.41061200 & -0.31383200 \\
\hline $\mathrm{C}$ & -5.62851400 & -0.41971100 & -0.96305100 & $\mathrm{O}$ & 1.09756400 & 0.33604600 & 1.07637500 \\
\hline $\mathrm{H}$ & -5.76298400 & 1.63115500 & -0.30687400 & $\mathrm{H}$ & 0.19443400 & 0.75920700 & 1.30974600 \\
\hline $\mathrm{H}$ & -3.43178700 & 1.66884400 & 0.61256800 & & & & \\
\hline $\mathrm{H}$ & -2.89591700 & -2.40557200 & -0.55793800 & \multirow{2}{*}{\multicolumn{4}{|c|}{$\begin{array}{l}\text { TS16 } \\
\text { imaginary frequency }=-161.92 \mathrm{~cm}^{-1}\end{array}$}} \\
\hline $\mathrm{H}$ & -5.18156400 & -2.46918200 & -1.47643500 & & & & \\
\hline $\mathrm{H}$ & -6.64172500 & -0.45150800 & -1.35929700 & $\mathrm{C}$ & -5.21516300 & 0.81814800 & 0.56984000 \\
\hline $\mathrm{C}$ & -1.60522900 & -0.22119700 & 0.64403700 & $\mathrm{C}$ & -4.52461200 & -0.31907200 & 0.15543200 \\
\hline $\mathrm{O}$ & -1.37592300 & 0.84635200 & 1.36231200 & $\mathrm{C}$ & -3.16583400 & -0.25133600 & -0.19022000 \\
\hline $\mathrm{C}$ & -0.65838100 & -1.18519900 & 0.39089500 & $\mathrm{C}$ & -2.51581000 & 0.99213500 & -0.14904700 \\
\hline $\mathrm{H}$ & -0.84935900 & -2.01403400 & -0.28005700 & $\mathrm{C}$ & -3.21660200 & 2.13533800 & 0.24828700 \\
\hline $\mathrm{C}$ & 2.60291500 & -3.91986500 & -0.71782200 & $\mathrm{C}$ & -4.56168100 & 2.05419700 & 0.61448600 \\
\hline $\mathrm{C}$ & 1.64248800 & -3.19731900 & -0.00980900 & $\mathrm{H}$ & -6.26501800 & 0.74609000 & 0.84891000 \\
\hline $\mathrm{C}$ & 1.78150000 & -1.81661200 & 0.20753500 & $\mathrm{H}$ & -5.02246500 & -1.28052600 & 0.07410800 \\
\hline $\mathrm{C}$ & 2.92019000 & -1.18098700 & -0.30443000 & $\mathrm{H}$ & -1.45774900 & 1.05127400 & -0.41253700 \\
\hline $\mathrm{C}$ & 3.88369200 & -1.90331800 & -1.01412400 & $\mathrm{H}$ & -2.70390900 & 3.09501900 & 0.27072100 \\
\hline $\mathrm{C}$ & 3.73400200 & -3.27464400 & -1.22453700 & $\mathrm{H}$ & -5.09992600 & 2.94680400 & 0.92856200 \\
\hline
\end{tabular}




\begin{tabular}{|c|c|c|c|c|c|c|c|}
\hline $\mathrm{C}$ & -2.51326300 & -1.51091800 & -0.73026200 & $\mathrm{C}$ & 2.50846900 & 0.13627900 & 0.00829600 \\
\hline $\mathrm{O}$ & -3.27039600 & -2.30242000 & -1.32939700 & $\mathrm{C}$ & 2.47138900 & -1.15793700 & 0.55103800 \\
\hline $\mathrm{C}$ & -1.09787400 & -1.78500000 & -0.64518900 & $\mathrm{C}$ & 3.63829400 & -1.91647000 & 0.65421500 \\
\hline $\mathrm{H}$ & -0.81447100 & -2.53856200 & -1.37728800 & $\mathrm{C}$ & 4.85397300 & -1.39652500 & 0.20675800 \\
\hline $\mathrm{C}$ & 2.30887700 & -4.30525400 & 0.08004300 & $\mathrm{H}$ & 5.84778200 & 0.30380200 & -0.67648300 \\
\hline $\mathrm{C}$ & 1.15054100 & -3.52478100 & 0.04926200 & $\mathrm{H}$ & 3.76075200 & 1.66353700 & -0.82015100 \\
\hline $\mathrm{C}$ & 1.20766200 & -2.12298400 & 0.14759900 & $\mathrm{H}$ & 1.54016400 & -1.57244200 & 0.92389000 \\
\hline $\mathrm{C}$ & 2.47311900 & -1.53056500 & 0.30522400 & $\mathrm{H}$ & 3.59711600 & -2.91265900 & 1.08639200 \\
\hline $\mathrm{C}$ & 3.62934400 & -2.31052300 & 0.33651000 & $\mathrm{H}$ & 5.76055700 & -1.99142800 & 0.28055100 \\
\hline $\mathrm{C}$ & 3.55799600 & -3.70134700 & 0.22180500 & $\mathrm{C}$ & 1.30024000 & 1.03057000 & -0.10747800 \\
\hline $\mathrm{H}$ & 2.22889400 & -5.38769200 & -0.00163700 & $\mathrm{O}$ & 1.47232900 & 2.24089200 & -0.25762400 \\
\hline $\mathrm{H}$ & 0.18130500 & -4.00526800 & -0.03993900 & $\mathrm{C}$ & -0.03216700 & 0.38545100 & -0.06778400 \\
\hline $\mathrm{H}$ & 2.54774200 & -0.45242400 & 0.36593800 & $\mathrm{H}$ & -0.02041700 & -0.69821200 & -0.10193200 \\
\hline $\mathrm{H}$ & 4.59536300 & -1.82116700 & 0.44184800 & $\mathrm{C}$ & -1.24881000 & 0.98781900 & -0.00658200 \\
\hline $\mathrm{H}$ & 4.46350700 & -4.30469700 & 0.24428800 & $\mathrm{C}$ & -1.44467700 & 2.47888400 & 0.12694100 \\
\hline $\mathrm{C}$ & -0.07783200 & -1.32269500 & 0.18966300 & $\mathrm{H}$ & -1.75190600 & 2.73253800 & 1.15121200 \\
\hline $\mathrm{C}$ & -0.32356500 & -0.72835700 & 1.56644000 & $\mathrm{H}$ & -0.52363100 & 3.01361300 & -0.09339500 \\
\hline $\mathrm{H}$ & -0.47303500 & -1.55142600 & 2.28132800 & $\mathrm{H}$ & -2.24567200 & 2.82110600 & -0.53819200 \\
\hline $\mathrm{H}$ & 0.54063800 & -0.14710100 & 1.89572700 & $\mathrm{C}$ & -2.47767500 & 0.14927600 & -0.03918400 \\
\hline $\mathrm{H}$ & -1.20458300 & -0.08982500 & 1.59724500 & $\mathrm{C}$ & -3.63207100 & 0.53513900 & 0.66703700 \\
\hline $\mathrm{C}$ & 2.56067800 & 3.12718000 & -0.17646200 & $\mathrm{C}$ & -2.53209100 & -1.04213400 & -0.78761300 \\
\hline $\mathrm{C}$ & 1.90606400 & 3.10659000 & 1.21960000 & $\mathrm{C}$ & -4.78134600 & -0.25408200 & 0.65298200 \\
\hline $\mathrm{H}$ & 2.58592300 & 2.64045800 & 1.94319700 & $\mathrm{H}$ & -3.62673000 & 1.45114000 & 1.24914800 \\
\hline $\mathrm{H}$ & 1.66042900 & 4.11703800 & 1.57553200 & $\mathrm{C}$ & -3.68368000 & -1.82572000 & -0.81039500 \\
\hline $\mathrm{H}$ & 0.98704300 & 2.51318500 & 1.18263600 & $\mathrm{H}$ & -1.67383900 & -1.33704100 & -1.38379700 \\
\hline $\mathrm{C}$ & 3.88763900 & 3.89793700 & -0.13287400 & $\mathrm{C}$ & -4.81285500 & -1.43856300 & -0.08494200 \\
\hline $\mathrm{H}$ & 4.57593000 & 3.42111800 & 0.57499400 & $\mathrm{H}$ & -5.65453500 & 0.05911700 & 1.21908300 \\
\hline $\mathrm{H}$ & 4.36101100 & 3.88690600 & -1.12178000 & $\mathrm{H}$ & -3.70312700 & -2.73481600 & -1.40555900 \\
\hline $\mathrm{H}$ & 3.73949600 & 4.94194500 & 0.17279000 & $\mathrm{H}$ & -5.71144300 & -2.04918000 & -0.10295600 \\
\hline $\mathrm{C}$ & 1.59826500 & 3.76873900 & -1.19708100 & & & & \\
\hline $\mathrm{H}$ & 2.06709700 & 3.79362900 & -2.18841500 & b3 & & & \\
\hline $\mathrm{H}$ & 0.69102200 & 3.15882200 & -1.26389100 & $\mathrm{C}$ & 1.03962200 & -1.23493200 & -0.30736900 \\
\hline $\mathrm{H}$ & 1.31764000 & 4.79411600 & -0.91860500 & $\mathrm{H}$ & 1.14049800 & -1.12828900 & -1.39959900 \\
\hline $\mathrm{O}$ & 2.88061400 & 1.81036800 & -0.58675900 & $\mathrm{H}$ & 1.53001600 & -2.18845400 & -0.04429400 \\
\hline $\mathrm{H}$ & 2.01111700 & 1.28205800 & -0.61781800 & $\mathrm{C}$ & -0.44693100 & -1.24901200 & 0.07463800 \\
\hline $\mathrm{O}$ & 0.59716800 & 0.49523100 & -0.56719600 & $\mathrm{H}$ & -1.00942700 & -1.94024200 & -0.56987500 \\
\hline \multirow[t]{2}{*}{$\mathrm{H}$} & 0.42022600 & 0.24022400 & -1.48742600 & $\mathrm{H}$ & -0.56284000 & -1.64200300 & 1.09933900 \\
\hline & & & & $\mathrm{C}$ & 1.75158100 & -0.05390100 & 0.36062000 \\
\hline aa2 & & & & $\mathrm{H}$ & 2.83545300 & -0.08401800 & 0.15614100 \\
\hline $\mathrm{C}$ & 4.90267800 & -0.10654600 & -0.33090100 & $\mathrm{H}$ & 1.62515500 & -0.13534100 & 1.45136700 \\
\hline $\mathrm{C}$ & 3.74137900 & 0.65518900 & -0.42019200 & $\mathrm{C}$ & 1.14003100 & 1.27022400 & -0.12499000 \\
\hline
\end{tabular}




$\begin{array}{rrrr}\mathrm{H} & 1.55280600 & 1.46106900 & -1.14659800 \\ \mathrm{H} & 1.56227500 & 2.09246300 & 0.48638300 \\ \mathrm{C} & -1.16835700 & 0.12970200 & 0.00557800 \\ \mathrm{C} & -0.36564100 & 1.26018000 & -0.06960800 \\ \mathrm{H} & -0.87384600 & 2.22543300 & -0.12179800 \\ \mathrm{O} & -2.43773900 & 0.07572700 & 0.05696400\end{array}$

\section{TS17} imaginary frequency $=-156.79 \mathrm{~cm}^{-1}$

C

$\mathrm{H}$

H

C

H

C

H

H

C

H

H

$\mathrm{C}$

C

H

$\mathrm{H}$

$\mathrm{O}$

C

C

C

C

C

C

H

H

H

H

H

C

O

C

H

H

\begin{tabular}{|c|c|c|}
\hline 0.85889800 & -1.31082600 & -1.08203600 \\
\hline 1.14134300 & -1.61126900 & -2.11048500 \\
\hline-0.18673300 & -1.63147700 & -0.96208800 \\
\hline 0.97412700 & 0.19927500 & -0.92523400 \\
\hline 0.41587600 & 0.77484700 & -1.66590600 \\
\hline 1.73634200 & -2.08760200 & -0.08591600 \\
\hline 1.72348600 & -3.16152800 & -0.32931200 \\
\hline 1.31218600 & -1.96320200 & 0.91607900 \\
\hline 3.17296600 & -1.55240300 & -0.08067200 \\
\hline 3.62783400 & -1.69440900 & -1.07481900 \\
\hline 3.79421900 & -2.12061500 & 0.62916500 \\
\hline 2.24820300 & 0.78235800 & -0.60801800 \\
\hline 3.17181900 & -0.05983800 & 0.28291300 \\
\hline 2.78516000 & 0.04634400 & 1.30917100 \\
\hline 4.17667900 & 0.37830800 & 0.24609600 \\
\hline 2.59016700 & 1.94814400 & -0.89873000 \\
\hline-3.44597400 & 0.64082600 & -1.04808100 \\
\hline-2.18882900 & 1.04491900 & -0.59800800 \\
\hline-1.48412700 & 0.29690000 & 0.36133100 \\
\hline-2.09367700 & -0.86779400 & 0.85606500 \\
\hline-3.34881500 & -1.27870600 & 0.40472800 \\
\hline-4.03606200 & -0.52601100 & -0.55037900 \\
\hline-3.96949500 & 1.24002900 & -1.79169300 \\
\hline-1.74603800 & 1.94909900 & -1.00505600 \\
\hline-1.54855000 & -1.41876000 & 1.61619400 \\
\hline-3.79685000 & -2.18744300 & 0.80494800 \\
\hline-5.01668100 & -0.84078800 & -0.90271400 \\
\hline-0.13278500 & 0.68954400 & 0.94945800 \\
\hline 0.30712100 & -0.01010900 & 1.90527600 \\
\hline 0.14340500 & 2.19740100 & 0.98996600 \\
\hline-0.02467400 & 2.71308800 & 0.04090800 \\
\hline-0.50344700 & 2.64756800 & 1.75896100 \\
\hline
\end{tabular}

$\mathrm{H}$

ba1

C

H

$\mathrm{H}$

C

H

C

$\mathrm{H}$

$\mathrm{H}$

C

$\mathrm{H}$

$\mathrm{H}$

$\mathrm{C}$

C

$\mathrm{H}$

$\mathrm{H}$

$\mathrm{O}$

C

C

$\mathrm{H}$

$\mathrm{H}$

$\mathrm{H}$

C

$\mathrm{H}$

$\mathrm{H}$

$\mathrm{H}$

C

$\mathrm{H}$

$\mathrm{H}$

$\mathrm{H}$

O

C

C

C

C

C

C

$\mathrm{H}$

H $\begin{array}{lll}1.18442900 & 2.36768100 & 1.27148400\end{array}$

\begin{tabular}{|c|c|c|}
\hline 3.108 & 0.336 & 11 \\
\hline 3.06633600 & -0.87002100 & .1417400 \\
\hline 3.16895600 & 0.73038200 & 14474270 \\
\hline 1.88223100 & -0.60517500 & 03105660 \\
\hline 2.18945200 & -0.58992600 & 07836120 \\
\hline 4.41935200 & -0.72958100 & 0.4753670 \\
\hline 5.25780500 & -0.67732500 & 1866170 \\
\hline 4.63905100 & -0.01182400 & 0.329 \\
\hline 4.30290400 & -2.13070000 & -0.13025800 \\
\hline 4.06100800 & -2.84371200 & 6726310 \\
\hline 5.26336300 & -2.45626900 & -0.5591340 \\
\hline 1.89932900 & -1.45466100 & -0.76734200 \\
\hline 3.19632500 & -2.15370500 & -1.18952600 \\
\hline 3.55455100 & 1.67888700 & 1010 \\
\hline 2.93317300 & 3.18324200 & 1.4669560 \\
\hline 0.87237600 & 1.73713600 & 15 \\
\hline 3.90294500 & 1.04178500 & -0.0556 \\
\hline-3.62211900 & -0.25414900 & \\
\hline-2.55491800 & -0.29567000 & 1.58906400 \\
\hline-4.18929500 & -0.65453600 & \\
\hline-3.89545600 & & \\
\hline .54068600 & 2.02 & \\
\hline-2.47942100 & -2.62994100 & \\
\hline-3.723 & -3.078 & \\
\hline-4.13109800 & -2.99231300 & 0700 \\
\hline 37468300 & -0.89797300 & \\
\hline 5.56309100 & -1.44519300 & \\
\hline-5.61562600 & & \\
\hline-6.04535500 & -1.28840500 & 95000 \\
\hline-3.14856700 & -0.49919200 & \\
\hline 1.32645400 & 3.52649600 & -0.8539620 \\
\hline 1.478422 & 2.209389 & -0.408 \\
\hline 0.421 & 1.52 & \\
\hline-0.81046300 & 2.1862 & \\
\hline-0.96796700 & 3.49891200 & $-0.12543 / 0$ \\
\hline 0.10386100 & 4.18198000 & -0.7089470 \\
\hline 2.1659 & 4.03614200 & -1.324548 \\
\hline & & \\
\hline
\end{tabular}




\begin{tabular}{|c|c|c|c|c|c|c|c|}
\hline $\mathrm{H}$ & -1.66263400 & 1.65487500 & 0.73375300 & $\mathrm{H}$ & -6.01689100 & -1.40911400 & -0.56752900 \\
\hline $\mathrm{H}$ & -1.93528300 & 3.98876500 & -0.02539100 & $\mathrm{O}$ & -3.20100900 & -0.52439100 & 0.99735800 \\
\hline $\mathrm{H}$ & -0.01884700 & 5.20600100 & -1.05731000 & $\mathrm{C}$ & 1.19380100 & 3.97865800 & -0.57130800 \\
\hline $\mathrm{C}$ & 0.56174500 & 0.06066900 & 0.72177300 & $\mathrm{C}$ & 1.61171600 & 2.72565900 & -0.11707800 \\
\hline $\mathrm{C}$ & 0.38717500 & 0.08313400 & 2.25786000 & $\mathrm{C}$ & 0.68497300 & 1.74887800 & 0.28664500 \\
\hline $\mathrm{H}$ & 1.14689200 & 0.69915800 & 2.75177400 & $\mathrm{C}$ & -0.68301200 & 2.06525600 & 0.22910500 \\
\hline $\mathrm{H}$ & -0.59880700 & 0.48189400 & 2.52152000 & $\mathrm{C}$ & -1.09786400 & 3.31704800 & -0.22680600 \\
\hline $\mathrm{H}$ & 0.45742400 & -0.94135600 & 2.63944800 & $\mathrm{C}$ & -0.16734200 & 4.28012400 & -0.62815200 \\
\hline $\mathrm{O}$ & -0.54773500 & -0.69390800 & 0.21706700 & $\mathrm{H}$ & 1.93316400 & 4.71652700 & -0.87755000 \\
\hline \multirow[t]{2}{*}{$\mathrm{H}$} & -0.15232300 & -1.17421900 & -0.62561300 & $\mathrm{H}$ & 2.67272100 & 2.49477600 & -0.06278800 \\
\hline & & & & $\mathrm{H}$ & -1.40277800 & 1.30575600 & 0.51166300 \\
\hline \multicolumn{4}{|c|}{ TS18 } & $\mathrm{H}$ & -2.16227500 & 3.53717300 & -0.27503100 \\
\hline \multicolumn{4}{|c|}{ imaginary frequency $=-73.36 \mathrm{~cm}^{-1}$} & $\mathrm{H}$ & -0.50039300 & 5.25339700 & -0.98386800 \\
\hline $\mathrm{C}$ & 2.11935500 & -0.21930400 & -1.35905300 & $\mathrm{C}$ & 1.21712600 & 0.47253500 & 0.87312400 \\
\hline $\mathrm{H}$ & 2.98099900 & 0.35700500 & -1.73919000 & $\mathrm{C}$ & 0.97498500 & 0.35705500 & 2.36071400 \\
\hline $\mathrm{H}$ & 1.21845000 & 0.30396300 & -1.68693200 & $\mathrm{H}$ & 1.69532800 & 0.98040200 & 2.91098800 \\
\hline $\mathrm{C}$ & 2.11845200 & -0.29595600 & 0.16583600 & $\mathrm{H}$ & -0.03323200 & 0.71004900 & 2.59083200 \\
\hline $\mathrm{H}$ & -2.17193400 & -0.65624600 & 0.79830300 & $\mathrm{H}$ & 1.10372500 & -0.66296500 & 2.72079000 \\
\hline $\mathrm{C}$ & 2.14953000 & -1.61113300 & -2.00125900 & $\mathrm{O}$ & -0.77089800 & -0.81113300 & 0.34251900 \\
\hline $\mathrm{H}$ & 2.12817200 & -1.52336600 & -3.09693100 & $\mathrm{H}$ & -0.40036700 & -1.54180900 & 0.86255600 \\
\hline $\mathrm{H}$ & 1.23945400 & -2.14472500 & -1.69494800 & & & & \\
\hline $\mathrm{C}$ & 3.39957400 & -2.36767100 & -1.54732600 & ba2 & & & \\
\hline $\mathrm{H}$ & 4.28982800 & -1.79688900 & -1.85376000 & $\mathrm{C}$ & 3.33867100 & -1.47628100 & 0.21051100 \\
\hline $\mathrm{H}$ & 3.47710800 & -3.34876500 & -2.03814200 & $\mathrm{C}$ & 2.06094000 & -0.98866000 & -0.08386900 \\
\hline $\mathrm{C}$ & 2.91180000 & -1.33831200 & 0.81427900 & $\mathrm{C}$ & 1.87881400 & 0.39388500 & -0.25370000 \\
\hline $\mathrm{C}$ & 3.39145900 & -2.54145500 & -0.02411000 & $\mathrm{C}$ & 2.97143600 & 1.26571400 & -0.12017100 \\
\hline $\mathrm{H}$ & 2.69781500 & -3.35546400 & 0.23848500 & $\mathrm{C}$ & 4.24462300 & 0.76723600 & 0.14484800 \\
\hline $\mathrm{H}$ & 4.37123800 & -2.84488200 & 0.36442600 & $\mathrm{C}$ & 4.43102600 & -0.61114200 & 0.30782900 \\
\hline $\mathrm{O}$ & 3.19677400 & -1.36039400 & 2.01996400 & $\mathrm{H}$ & 3.48127000 & -2.54478700 & 0.36442400 \\
\hline $\mathrm{C}$ & -3.89402800 & -1.13205900 & -0.06827300 & $\mathrm{H}$ & 1.16689600 & -1.63501800 & -0.09433000 \\
\hline $\mathrm{C}$ & -3.52299100 & -0.45324000 & -1.40529800 & $\mathrm{H}$ & 2.79150800 & 2.33172800 & -0.22758500 \\
\hline $\mathrm{H}$ & -2.43921700 & -0.52484400 & -1.54224300 & $\mathrm{H}$ & 5.09129200 & 1.44650200 & 0.23152600 \\
\hline $\mathrm{H}$ & -4.03085900 & -0.91555300 & -2.26485800 & $\mathrm{H}$ & 5.42551200 & -1.00462600 & 0.51465600 \\
\hline $\mathrm{H}$ & -3.79633000 & 0.60925600 & -1.37337900 & $\mathrm{C}$ & 0.52389600 & 0.97750400 & -0.55801500 \\
\hline $\mathrm{C}$ & -3.52798800 & -2.63184900 & -0.14213100 & $\mathrm{O}$ & 0.34066600 & 2.18921100 & -0.37910700 \\
\hline $\mathrm{H}$ & -2.44622500 & -2.71316200 & -0.29373300 & $\mathrm{C}$ & -0.55931200 & 0.08467200 & -1.06933300 \\
\hline $\mathrm{H}$ & -3.78311100 & -3.12525300 & 0.80450600 & $\mathrm{H}$ & -0.16851300 & -0.64791300 & -1.78249200 \\
\hline $\mathrm{H}$ & -4.04710000 & -3.15473100 & -0.95882300 & $\mathrm{H}$ & -1.27405000 & 0.73914000 & -1.57746600 \\
\hline $\mathrm{C}$ & -5.39404100 & -0.96262400 & 0.22009900 & $\mathrm{C}$ & -2.68589000 & 1.12046400 & 1.08519600 \\
\hline $\mathrm{H}$ & -5.64811500 & -1.43606500 & 1.17650400 & $\mathrm{H}$ & -2.23286800 & 1.87839300 & 0.43227500 \\
\hline $\mathrm{H}$ & -5.64114800 & 0.10322100 & 0.29788900 & $\mathrm{H}$ & -2.90793700 & 1.62761000 & 2.03814300 \\
\hline
\end{tabular}




\begin{tabular}{|c|c|c|c|c|c|c|c|}
\hline $\mathrm{C}$ & -1.68771800 & -0.02794400 & 1.30560700 & $\mathrm{H}$ & -3.14148000 & 1.67984400 & 0.60977600 \\
\hline $\mathrm{H}$ & -0.75568700 & 0.34897200 & 1.74606400 & $\mathrm{C}$ & -2.38933800 & -0.07014800 & 1.63302300 \\
\hline $\mathrm{H}$ & -2.10273200 & -0.74341400 & 2.03204800 & $\mathrm{H}$ & -2.64454200 & -1.13475200 & 1.72826900 \\
\hline C & -3.99243400 & 0.60640200 & 0.45934900 & $\mathrm{H}$ & -2.50024500 & 0.38240800 & 2.63093700 \\
\hline $\mathrm{H}$ & -4.69497900 & 1.43698400 & 0.28841600 & $\mathrm{C}$ & -0.83726000 & -0.65545300 & -0.24603600 \\
\hline $\mathrm{H}$ & -4.48411100 & -0.07693200 & 1.17067900 & $\mathrm{C}$ & -0.93253500 & 0.03234400 & 1.13856200 \\
\hline $\mathrm{C}$ & -3.72807600 & -0.14796900 & -0.85364300 & $\mathrm{H}$ & -0.64150400 & 1.08900000 & 1.08394400 \\
\hline $\mathrm{H}$ & -3.37613500 & 0.57109800 & -1.60821400 & $\mathrm{H}$ & -0.26366200 & -0.47996200 & 1.84067600 \\
\hline $\mathrm{H}$ & -4.67309700 & -0.55711400 & -1.24739600 & $\mathrm{O}$ & -0.86461500 & -1.92376700 & -0.25802600 \\
\hline $\mathrm{C}$ & -1.33563300 & -0.88708100 & 0.04099600 & & & & \\
\hline $\mathrm{C}$ & -2.69365900 & -1.27141800 & -0.66460900 & bb1 & & & \\
\hline $\mathrm{H}$ & -3.12327300 & -2.05610700 & -0.02460500 & C & -1.50856400 & 1.65016600 & -1.36375700 \\
\hline $\mathrm{H}$ & -2.45943900 & -1.75355300 & -1.62469500 & $\mathrm{H}$ & -2.61964200 & 1.65490700 & -1.36266500 \\
\hline \multirow[t]{2}{*}{$\mathrm{O}$} & -0.62863500 & -1.96336800 & 0.33692800 & $\mathrm{H}$ & -1.23743900 & 1.09496600 & -2.27529500 \\
\hline & & & & $\mathrm{C}$ & -0.94838200 & 0.97838900 & -0.12568000 \\
\hline \multicolumn{4}{|c|}{ TS19 } & $\mathrm{C}$ & -1.02962400 & 3.09964900 & -1.53904200 \\
\hline \multicolumn{4}{|c|}{ imaginary frequency $=-132.91 \mathrm{~cm}^{-1}$} & $\mathrm{H}$ & -1.60774000 & 3.59508400 & -2.33411900 \\
\hline $\mathrm{C}$ & 1.87962000 & -1.31689700 & -0.81092600 & $\mathrm{H}$ & 0.02166400 & 3.09690800 & -1.86336200 \\
\hline $\mathrm{H}$ & 2.60963300 & -1.44129300 & -1.63225600 & $\mathrm{C}$ & -1.13556000 & 3.87176000 & -0.22227100 \\
\hline $\mathrm{H}$ & 1.21098600 & -2.19198200 & -0.83113900 & $\mathrm{H}$ & -2.18292900 & 3.85747000 & 0.11666600 \\
\hline $\mathrm{C}$ & 1.01632800 & -0.08962400 & -1.02952900 & $\mathrm{H}$ & -0.86567400 & 4.92994200 & -0.36302600 \\
\hline $\mathrm{H}$ & 0.63558500 & -0.00622300 & -2.04771400 & $\mathrm{C}$ & -0.39552800 & 1.69227100 & 0.91862600 \\
\hline C & 2.64284300 & -1.29256800 & 0.52120200 & $\mathrm{C}$ & -0.24422300 & 3.21480200 & 0.83561800 \\
\hline $\mathrm{H}$ & 3.35642200 & -2.12943200 & 0.56739100 & $\mathrm{H}$ & 0.81336500 & 3.45379500 & 0.63820400 \\
\hline $\mathrm{H}$ & 1.93027200 & -1.44244800 & 1.34436100 & $\mathrm{H}$ & -0.45680300 & 3.61181400 & 1.83723500 \\
\hline $\mathrm{C}$ & 3.36876000 & 0.04317000 & 0.71810200 & $\mathrm{O}$ & 0.06051200 & 1.16920100 & 2.02556000 \\
\hline $\mathrm{H}$ & 4.09242300 & 0.18200700 & -0.10080800 & $\mathrm{C}$ & -1.43240800 & -2.84279700 & -0.97202500 \\
\hline $\mathrm{H}$ & 3.95340100 & 0.04281700 & 1.65186300 & $\mathrm{H}$ & -0.63465100 & -3.25332800 & -0.34194900 \\
\hline $\mathrm{C}$ & 1.38827900 & 1.18592500 & -0.48596800 & $\mathrm{H}$ & -1.40550000 & -3.39408500 & -1.92375300 \\
\hline C & 2.36297300 & 1.20298700 & 0.71300500 & $\mathrm{C}$ & -1.14718500 & -1.35305300 & -1.22185200 \\
\hline $\mathrm{H}$ & 1.76394900 & 1.16686100 & 1.63612400 & $\mathrm{H}$ & -0.15990500 & -1.23334600 & -1.68416400 \\
\hline $\mathrm{H}$ & 2.86371700 & 2.17927100 & 0.70718800 & $\mathrm{H}$ & -1.89205300 & -0.96307200 & -1.92925500 \\
\hline $\mathrm{O}$ & 0.90874300 & 2.28196000 & -0.84994500 & $\mathrm{C}$ & -2.78843000 & -3.04829100 & -0.27897500 \\
\hline $\mathrm{C}$ & -3.19891700 & 0.01868900 & -0.77553400 & $\mathrm{H}$ & -2.95903900 & -4.11680500 & -0.07859400 \\
\hline $\mathrm{H}$ & -3.50070800 & -1.03838800 & -0.76533300 & $\mathrm{H}$ & -3.59508600 & -2.72578300 & -0.95810400 \\
\hline $\mathrm{H}$ & -3.87212400 & 0.54141300 & -1.47298000 & $\mathrm{C}$ & -2.86800500 & -2.23677700 & 1.02293400 \\
\hline $\mathrm{C}$ & -1.73726600 & 0.09264700 & -1.26065000 & $\mathrm{H}$ & -2.12707000 & -2.62539900 & 1.73225900 \\
\hline $\mathrm{H}$ & -1.64582600 & -0.40102200 & -2.23608300 & $\mathrm{H}$ & -3.85871800 & -2.35706700 & 1.48761800 \\
\hline $\mathrm{H}$ & -1.41605900 & 1.13645600 & -1.36965200 & C & -1.18848600 & -0.52297800 & 0.08099700 \\
\hline $\mathrm{C}$ & -3.35479700 & 0.59965000 & 0.64037500 & C & -2.56388100 & -0.75087000 & 0.77716600 \\
\hline $\mathrm{H}$ & -4.39540500 & 0.49555100 & 0.98545200 & $\mathrm{H}$ & -3.35740500 & -0.30676400 & 0.15514800 \\
\hline
\end{tabular}




\begin{tabular}{|c|c|c|c|c|c|c|c|}
\hline $\mathrm{H}$ & -2.55005900 & -0.19981100 & 1.72473400 & $\mathrm{H}$ & -1.50550300 & 2.16827100 & 2.08387600 \\
\hline $\mathrm{C}$ & 3.19851800 & -0.66755800 & -0.13015100 & $\mathrm{C}$ & 0.27460900 & 3.55016100 & 0.31637000 \\
\hline $\mathrm{C}$ & 3.26842000 & -0.05546900 & 1.28200900 & $\mathrm{H}$ & 1.20576200 & 4.10808100 & 0.14357000 \\
\hline $\mathrm{H}$ & 3.50879400 & -0.83844900 & 2.01221200 & $\mathrm{H}$ & -0.46477200 & 4.27848300 & 0.69220500 \\
\hline $\mathrm{H}$ & 4.04591800 & 0.71924200 & 1.34160500 & $\mathrm{C}$ & -0.22891400 & 2.93073900 & -0.99051200 \\
\hline $\mathrm{H}$ & 2.31151100 & 0.40077800 & 1.56455200 & $\mathrm{H}$ & 0.52405300 & 2.22256900 & -1.35288300 \\
\hline $\mathrm{C}$ & 4.55289600 & -1.28174900 & -0.51365100 & $\mathrm{H}$ & -0.38354300 & 3.70505300 & -1.75621100 \\
\hline $\mathrm{H}$ & 4.83878200 & -2.05025100 & 0.21431300 & $\mathrm{C}$ & -1.43631200 & 1.09381000 & 0.28724800 \\
\hline $\mathrm{H}$ & 4.48462900 & -1.75878800 & -1.49880400 & $\mathrm{C}$ & -1.54291800 & 2.15481900 & -0.78877500 \\
\hline $\mathrm{H}$ & 5.34272700 & -0.52057300 & -0.54796800 & $\mathrm{H}$ & -2.34275400 & 2.85885800 & -0.49793200 \\
\hline $\mathrm{C}$ & 2.79238900 & 0.40778800 & -1.15719300 & $\mathrm{H}$ & -1.88023800 & 1.71368400 & -1.72538000 \\
\hline $\mathrm{H}$ & 2.75362500 & -0.03126200 & -2.16207000 & $\mathrm{C}$ & 3.92973400 & -0.93927400 & -0.25868300 \\
\hline $\mathrm{H}$ & 1.79820100 & 0.80137300 & -0.91510900 & $\mathrm{C}$ & 3.28196500 & -2.20276300 & -0.87058300 \\
\hline $\mathrm{H}$ & 3.50402500 & 1.24538100 & -1.17246400 & $\mathrm{H}$ & 3.43885000 & -2.21319200 & -1.95702800 \\
\hline $\mathrm{O}$ & 2.27481600 & -1.74952300 & -0.16391700 & $\mathrm{H}$ & 3.68918400 & -3.13468200 & -0.45101100 \\
\hline $\mathrm{H}$ & 1.40724400 & -1.44966300 & 0.21349000 & $\mathrm{H}$ & 2.20485800 & -2.15791500 & -0.67679900 \\
\hline $\mathrm{O}$ & -0.17293900 & -1.07886600 & 0.94198900 & $\mathrm{C}$ & 5.43253400 & -0.90094000 & -0.58007700 \\
\hline $\mathrm{H}$ & 0.00843100 & -0.29540200 & 1.58707800 & $\mathrm{H}$ & 5.58230400 & -0.87860900 & -1.66658600 \\
\hline & & & & $\mathrm{H}$ & 5.88088900 & 0.00862600 & -0.16168200 \\
\hline & & & & $\mathrm{H}$ & 5.96345000 & -1.77229400 & -0.17160600 \\
\hline & uency $=-48$ & $.72 \mathrm{~cm}^{-1}$ & & C & 3.70946200 & -0.94674900 & 1.27117000 \\
\hline $\mathrm{C}$ & -1.74834400 & -1.17345100 & 1.28523600 & $\mathrm{H}$ & 4.16836100 & -0.05482600 & 1.71653900 \\
\hline $\mathrm{H}$ & -2.20468300 & -0.95575100 & 2.26605800 & $\mathrm{H}$ & 2.63244900 & -0.91224600 & 1.46447500 \\
\hline $\mathrm{H}$ & -0.66033300 & -1.12591500 & 1.39967600 & $\mathrm{H}$ & 4.13692000 & -1.83732800 & 1.75612700 \\
\hline $\mathrm{C}$ & -2.12218900 & -0.10346200 & 0.25593700 & $\mathrm{O}$ & 3.36049600 & 0.21557700 & -0.82296300 \\
\hline $\mathrm{C}$ & -2.12833100 & -2.60562500 & 0.89011700 & $\mathrm{H}$ & 2.32531300 & 0.16566700 & -0.62900500 \\
\hline $\mathrm{H}$ & -1.95293200 & -3.27908700 & 1.74047400 & $\mathrm{O}$ & 0.89347000 & -0.01898000 & -0.25265900 \\
\hline $\mathrm{H}$ & -1.46785500 & -2.94476400 & 0.07878800 & $\mathrm{H}$ & 0.46150300 & -0.39426500 & -1.03671300 \\
\hline $\mathrm{C}$ & -3.57886800 & -2.67989900 & 0.41744000 & & & & \\
\hline $\mathrm{H}$ & -4.24377300 & -2.31288800 & 1.21427700 & bb2 & & & \\
\hline $\mathrm{H}$ & -3.88277800 & -3.71574600 & 0.20719500 & $\mathrm{C}$ & 1.35527200 & -1.44769600 & -0.38290600 \\
\hline $\mathrm{C}$ & -3.16167300 & -0.38646400 & -0.73833800 & $\mathrm{H}$ & 1.45249700 & -1.50097900 & -1.47928200 \\
\hline $\mathrm{C}$ & -3.73417800 & -1.81479500 & -0.83417300 & $\mathrm{H}$ & 0.82001600 & -2.35153000 & -0.08294500 \\
\hline $\mathrm{H}$ & -3.20685400 & -2.30031700 & -1.67072600 & C & 0.56690200 & -0.19290800 & -0.01702200 \\
\hline $\mathrm{H}$ & -4.77948400 & -1.71496100 & -1.14865000 & C & 2.76996600 & -1.47170900 & 0.22160000 \\
\hline $\mathrm{O}$ & -3.64710300 & 0.44214700 & -1.52230800 & $\mathrm{H}$ & 3.29173300 & -2.37881100 & -0.10829700 \\
\hline C & 0.50804800 & 2.44607500 & 1.35280800 & $\mathrm{H}$ & 2.69994100 & -1.52541100 & 1.31732700 \\
\hline $\mathrm{H}$ & 1.31183300 & 1.79474700 & 1.00285100 & $\mathrm{C}$ & 3.55780600 & -0.22348500 & -0.18302900 \\
\hline $\mathrm{H}$ & 0.79837100 & 2.88078900 & 2.32134900 & $\mathrm{H}$ & 3.68335800 & -0.20980500 & -1.27494200 \\
\hline $\mathrm{C}$ & -0.73965000 & 1.57027700 & 1.55020000 & $\mathrm{H}$ & 4.56641800 & -0.23904100 & 0.24793900 \\
\hline $\mathrm{H}$ & -0.49335200 & 0.73524300 & 2.20588100 & $\mathrm{C}$ & 1.34598000 & 1.08698500 & -0.14594200 \\
\hline
\end{tabular}




\begin{tabular}{|c|c|c|c|c|c|c|c|}
\hline $\mathrm{C}$ & 2.81837100 & 1.04599800 & 0.26481700 & $\mathrm{C}$ & 3.51676600 & -3.35289900 & -0.39182400 \\
\hline $\mathrm{H}$ & 2.83385400 & 1.09541400 & 1.36546700 & $\mathrm{C}$ & 3.28942500 & -2.12134100 & 0.24031900 \\
\hline $\mathrm{H}$ & 3.29293100 & 1.95950900 & -0.10620600 & $\mathrm{C}$ & 0.64940300 & 4.48349300 & 0.64681900 \\
\hline $\mathrm{O}$ & 0.85318000 & 2.13698100 & -0.53660700 & $\mathrm{C}$ & 0.85797900 & 5.83290100 & 0.94101800 \\
\hline $\mathrm{C}$ & -2.72305800 & 1.23587500 & -0.16315500 & $\mathrm{C}$ & -0.21426900 & 6.72574200 & 0.94092500 \\
\hline $\mathrm{H}$ & -2.34437600 & 1.44678100 & -1.17183400 & $\mathrm{C}$ & -1.50018400 & 6.26494800 & 0.64747800 \\
\hline $\mathrm{H}$ & -3.29721000 & 2.11617500 & 0.15211300 & $\mathrm{C}$ & -1.71135300 & 4.91706500 & 0.35814700 \\
\hline $\mathrm{C}$ & -1.51619000 & 1.04098300 & 0.78146500 & $\mathrm{C}$ & -0.63697600 & 4.01149800 & 0.35016500 \\
\hline $\mathrm{H}$ & -0.90166800 & 1.93864800 & 0.78688200 & $\mathrm{C}$ & -3.72007200 & 1.69556600 & -0.14546700 \\
\hline $\mathrm{H}$ & -1.90200200 & 0.88175800 & 1.80157200 & $\mathrm{C}$ & -4.94892700 & 1.43414900 & 0.46869100 \\
\hline $\mathrm{C}$ & -2.81470900 & -1.27768700 & -0.49480300 & $\mathrm{C}$ & -5.04397700 & 1.39896900 & 1.85926700 \\
\hline $\mathrm{H}$ & -2.44897200 & -1.23745900 & -1.53010100 & $\mathrm{C}$ & -3.90248900 & 1.61873200 & 2.63684400 \\
\hline $\mathrm{H}$ & -3.45235500 & -2.16761600 & -0.41747300 & $\mathrm{C}$ & -2.67563100 & 1.86556400 & 2.02384500 \\
\hline $\mathrm{C}$ & -0.72872100 & -0.19329800 & 0.38602400 & $\mathrm{C}$ & -2.56902800 & 1.91606000 & 0.62280400 \\
\hline $\mathrm{C}$ & -1.60113600 & -1.43115700 & 0.45495700 & $\mathrm{H}$ & 2.96957300 & -1.38972900 & -2.99392400 \\
\hline $\mathrm{H}$ & -1.97839000 & -1.54390600 & 1.48319900 & $\mathrm{H}$ & 1.60232400 & -2.16434500 & -2.16717900 \\
\hline $\mathrm{H}$ & -1.06346400 & -2.34994500 & 0.21186700 & $\mathrm{H}$ & 1.65456100 & 0.75526600 & -3.11585900 \\
\hline $\mathrm{C}$ & -3.62119300 & -0.00881300 & -0.18528400 & $\mathrm{H}$ & 0.76045600 & -0.58675900 & -3.86916700 \\
\hline $\mathrm{H}$ & -4.42824400 & 0.11339700 & -0.91915500 & $\mathrm{H}$ & -2.02775000 & 2.81447600 & -2.24649600 \\
\hline \multirow[t]{2}{*}{$\mathrm{H}$} & -4.10524700 & -0.12156000 & 0.79639700 & $\mathrm{H}$ & -0.26259100 & 2.89110800 & -2.29448800 \\
\hline & & & & $\mathrm{H}$ & -1.99517400 & 0.35487500 & -2.31557900 \\
\hline \multirow{2}{*}{\multicolumn{3}{|c|}{$\begin{array}{l}\text { TS21 } \\
\text { imaginary frequency }=-462.66 \mathrm{~cm}^{-1}\end{array}$}} & & $\mathrm{H}$ & -0.96726300 & 0.93467300 & -3.62055000 \\
\hline & & & & $\mathrm{H}$ & 3.67923300 & 2.16024700 & -0.34987800 \\
\hline $\mathrm{Ru}$ & 0.85098900 & 0.68210900 & 0.04876400 & $\mathrm{H}$ & 5.85176400 & 3.26321000 & -0.84215700 \\
\hline $\mathrm{P}$ & 2.69754000 & -0.60750800 & -0.63889400 & $\mathrm{H}$ & 7.72256100 & 1.94539600 & -1.81771200 \\
\hline$P$ & -0.89494300 & 2.23390900 & -0.08774900 & $\mathrm{H}$ & 7.41688900 & -0.48385800 & -2.26442700 \\
\hline $\mathrm{N}$ & 0.04190300 & 0.04442900 & -1.96677900 & $\mathrm{H}$ & 5.27970600 & -1.58309100 & -1.72710600 \\
\hline $\mathrm{C}$ & 2.12785400 & -1.21383900 & -2.31587400 & $\mathrm{H}$ & 3.33419000 & -1.07952300 & 2.13362700 \\
\hline $\mathrm{C}$ & 1.15312700 & -0.19760000 & -2.91751800 & $\mathrm{H}$ & 4.16252700 & -3.01909700 & 3.41577100 \\
\hline $\mathrm{C}$ & -1.11093300 & 2.29607600 & -1.94121400 & $\mathrm{H}$ & 4.55637300 & -5.19719700 & 2.27623200 \\
\hline $\mathrm{C}$ & -1.06185900 & 0.88273500 & -2.52780800 & $\mathrm{H}$ & 4.13408100 & -5.40334900 & -0.16598100 \\
\hline $\mathrm{C}$ & 4.50373700 & 1.58629700 & -0.75928700 & $\mathrm{H}$ & 3.33842700 & -3.46693300 & -1.45700500 \\
\hline $\mathrm{C}$ & 5.72472500 & 2.20318000 & -1.04635500 & $\mathrm{H}$ & 1.48134800 & 3.78747200 & 0.64122000 \\
\hline $\mathrm{C}$ & 6.77401700 & 1.46482000 & -1.59256400 & $\mathrm{H}$ & 1.85980200 & 6.18323300 & 1.17533400 \\
\hline $\mathrm{C}$ & 6.60233400 & 0.10080200 & -1.84472900 & $\mathrm{H}$ & -0.05140400 & 7.77509400 & 1.17267900 \\
\hline $\mathrm{C}$ & 5.38785300 & -0.51645800 & -1.54963800 & $\mathrm{H}$ & -2.34063800 & 6.95417900 & 0.64870000 \\
\hline $\mathrm{C}$ & 4.31915400 & 0.22115800 & -1.01091200 & $\mathrm{H}$ & -2.71800100 & 4.56736500 & 0.14748600 \\
\hline $\mathrm{C}$ & 3.52555500 & -2.01859500 & 1.62159800 & $\mathrm{H}$ & -3.67474800 & 1.71958200 & -1.22958700 \\
\hline $\mathrm{C}$ & 3.98505700 & -3.11669500 & 2.34810800 & $\mathrm{H}$ & -5.82666400 & 1.25152800 & -0.14482800 \\
\hline C & 4.20594400 & -4.33938600 & 1.70865000 & $\mathrm{H}$ & -5.99949500 & 1.19891800 & 2.33665700 \\
\hline $\mathrm{C}$ & 3.96903000 & -4.45498100 & 0.33868900 & $\mathrm{H}$ & -3.96741100 & 1.59244100 & 3.72142000 \\
\hline
\end{tabular}




\begin{tabular}{|c|c|c|c|c|c|c|c|}
\hline $\mathrm{H}$ & -1.79146000 & 2.01978400 & 2.63747000 & $\mathrm{P}$ & 2.69754000 & -0.60750800 & -0.63889400 \\
\hline $\mathrm{C}$ & 1.47310200 & 1.11256400 & 1.73648200 & $\mathrm{P}$ & -0.89494300 & 2.23390900 & -0.08774900 \\
\hline $\mathrm{O}$ & 1.85673100 & 1.35726000 & 2.81203100 & $\mathrm{~N}$ & 0.04190300 & 0.04442900 & -1.96677900 \\
\hline $\mathrm{H}$ & -0.13503900 & -0.65474900 & 0.68472800 & $\mathrm{C}$ & 2.12785400 & -1.21383900 & -2.31587400 \\
\hline $\mathrm{H}$ & 1.64520500 & 1.92636400 & -0.72606200 & $\mathrm{C}$ & 1.15312700 & -0.19760000 & -2.91751800 \\
\hline $\mathrm{H}$ & -0.39241200 & -0.86942100 & -1.76744100 & $\mathrm{C}$ & -1.11093300 & 2.29607600 & -1.94121400 \\
\hline $\mathrm{C}$ & -1.54371700 & -2.94377600 & 3.41912900 & $\mathrm{C}$ & -1.06185900 & 0.88273500 & -2.52780800 \\
\hline $\mathrm{H}$ & -2.58543300 & -3.19851000 & 3.18083300 & $\mathrm{C}$ & 4.50373700 & 1.58629700 & -0.75928700 \\
\hline $\mathrm{H}$ & -1.50247200 & -2.74713000 & 4.49881600 & $\mathrm{C}$ & 5.72472500 & 2.20318000 & -1.04635500 \\
\hline $\mathrm{C}$ & -1.14259100 & -1.66541600 & 2.64772300 & $\mathrm{C}$ & 6.77401700 & 1.46482000 & -1.59256400 \\
\hline $\mathrm{H}$ & -1.82691800 & -0.84395000 & 2.88443000 & $\mathrm{C}$ & 6.60233400 & 0.10080200 & -1.84472900 \\
\hline $\mathrm{H}$ & -0.14008000 & -1.35802800 & 2.96594700 & $\mathrm{C}$ & 5.38785300 & -0.51645800 & -1.54963800 \\
\hline $\mathrm{C}$ & -0.63040800 & -4.12346700 & 3.05350200 & $\mathrm{C}$ & 4.31915400 & 0.22115800 & -1.01091200 \\
\hline $\mathrm{H}$ & -0.96314600 & -5.03487500 & 3.56798700 & $\mathrm{C}$ & 3.52555500 & -2.01859500 & 1.62159800 \\
\hline $\mathrm{H}$ & 0.38845700 & -3.91432000 & 3.41210500 & $\mathrm{C}$ & 3.98505700 & -3.11669500 & 2.34810800 \\
\hline $\mathrm{C}$ & -0.58824000 & -4.35054500 & 1.53514200 & $\mathrm{C}$ & 4.20594400 & -4.33938600 & 1.70865000 \\
\hline $\mathrm{H}$ & -1.57372500 & -4.68223300 & 1.18233000 & $\mathrm{C}$ & 3.96903000 & -4.45498100 & 0.33868900 \\
\hline $\mathrm{H}$ & 0.12486400 & -5.14873300 & 1.29070700 & $\mathrm{C}$ & 3.51676600 & -3.35289900 & -0.39182400 \\
\hline $\mathrm{C}$ & -1.13849200 & -1.93814400 & 1.14791300 & $\mathrm{C}$ & 3.28942500 & -2.12134100 & 0.24031900 \\
\hline $\mathrm{C}$ & -0.17962700 & -3.06350300 & 0.77903300 & $\mathrm{C}$ & 0.64940300 & 4.48349300 & 0.64681900 \\
\hline $\mathrm{H}$ & 0.83513300 & -2.79216700 & 1.07992500 & $\mathrm{C}$ & 0.85797900 & 5.83290100 & 0.94101800 \\
\hline $\mathrm{H}$ & -0.18622100 & -3.23646500 & -0.29738400 & $\mathrm{C}$ & -0.21426900 & 6.72574200 & 0.94092500 \\
\hline $\mathrm{C}$ & -6.42626200 & -2.41194900 & -1.01385200 & $\mathrm{C}$ & -1.50018400 & 6.26494800 & 0.64747800 \\
\hline $\mathrm{C}$ & -5.12763500 & -2.32566100 & -0.51111900 & $\mathrm{C}$ & -1.71135300 & 4.91706500 & 0.35814700 \\
\hline $\mathrm{C}$ & -4.03118200 & -2.18807000 & -1.37751500 & $\mathrm{C}$ & -0.63697600 & 4.01149800 & 0.35016500 \\
\hline $\mathrm{C}$ & -4.27027000 & -2.16029900 & -2.76062800 & $\mathrm{C}$ & -3.72007200 & 1.69556600 & -0.14546700 \\
\hline $\mathrm{C}$ & -5.56665100 & -2.24219000 & -3.26491800 & $\mathrm{C}$ & -4.94892700 & 1.43414900 & 0.46869100 \\
\hline $\mathrm{C}$ & -6.65118600 & -2.36502000 & -2.39192200 & $\mathrm{C}$ & -5.04397700 & 1.39896900 & 1.85926700 \\
\hline $\mathrm{H}$ & -7.26319800 & -2.52548600 & -0.32926100 & $\mathrm{C}$ & -3.90248900 & 1.61873200 & 2.63684400 \\
\hline $\mathrm{H}$ & -4.96358100 & -2.38807800 & 0.55966500 & $\mathrm{C}$ & -2.67563100 & 1.86556400 & 2.02384500 \\
\hline $\mathrm{H}$ & -3.41386400 & -2.08217300 & -3.42236800 & $\mathrm{C}$ & -2.56902800 & 1.91606000 & 0.62280400 \\
\hline $\mathrm{H}$ & -5.73347900 & -2.21504700 & -4.33901700 & $\mathrm{H}$ & 2.96957300 & -1.38972900 & -2.99392400 \\
\hline $\mathrm{H}$ & -7.66335600 & -2.43321800 & -2.78267600 & $\mathrm{H}$ & 1.60232400 & -2.16434500 & -2.16717900 \\
\hline $\mathrm{C}$ & -2.60226800 & -2.10010300 & -0.89693100 & $\mathrm{H}$ & 1.65456100 & 0.75526600 & -3.11585900 \\
\hline $\mathrm{O}$ & -1.69450800 & -2.25286300 & -1.75937100 & $\mathrm{H}$ & 0.76045600 & -0.58675900 & -3.86916700 \\
\hline $\mathrm{C}$ & -2.36052900 & -1.79564800 & 0.47930800 & $\mathrm{H}$ & -2.02775000 & 2.81447600 & -2.24649600 \\
\hline \multirow[t]{2}{*}{$\mathrm{H}$} & -3.16735100 & -1.31217200 & 1.02312200 & $\mathrm{H}$ & -0.26259100 & 2.89110800 & -2.29448800 \\
\hline & & & & $\mathrm{H}$ & -1.99517400 & 0.35487500 & -2.31557900 \\
\hline \multicolumn{4}{|c|}{ TS21-D } & $\mathrm{H}$ & -0.96726300 & 0.93467300 & -3.62055000 \\
\hline \multicolumn{4}{|c|}{ imaginary frequency $=-418.20 \mathrm{~cm}^{-1}$} & $\mathrm{H}$ & 3.67923300 & 2.16024700 & -0.34987800 \\
\hline $\mathrm{Ru}$ & 0.85098900 & 0.68210900 & 0.04876400 & $\mathrm{H}$ & 5.85176400 & 3.26321000 & -0.84215700 \\
\hline
\end{tabular}




\begin{tabular}{|c|c|c|c|c|c|c|c|}
\hline $\mathrm{H}$ & 7.72256100 & 1.94539600 & -1.81771200 & $\mathrm{C}$ & -4.03118200 & -2.18807000 & -1.37751500 \\
\hline $\mathrm{H}$ & 7.41688900 & -0.48385800 & -2.26442700 & $\mathrm{C}$ & -4.27027000 & -2.16029900 & -2.76062800 \\
\hline $\mathrm{H}$ & 5.27970600 & -1.58309100 & -1.72710600 & $\mathrm{C}$ & -5.56665100 & -2.24219000 & -3.26491800 \\
\hline $\mathrm{H}$ & 3.33419000 & -1.07952300 & 2.13362700 & $\mathrm{C}$ & -6.65118600 & -2.36502000 & -2.39192200 \\
\hline $\mathrm{H}$ & 4.16252700 & -3.01909700 & 3.41577100 & $\mathrm{H}$ & -7.26319800 & -2.52548600 & -0.32926100 \\
\hline $\mathrm{H}$ & 4.55637300 & -5.19719700 & 2.27623200 & $\mathrm{H}$ & -4.96358100 & -2.38807800 & 0.55966500 \\
\hline $\mathrm{H}$ & 4.13408100 & -5.40334900 & -0.16598100 & $\mathrm{H}$ & -3.41386400 & -2.08217300 & -3.42236800 \\
\hline $\mathrm{H}$ & 3.33842700 & -3.46693300 & -1.45700500 & $\mathrm{H}$ & -5.73347900 & -2.21504700 & -4.33901700 \\
\hline $\mathrm{H}$ & 1.48134800 & 3.78747200 & 0.64122000 & $\mathrm{H}$ & -7.66335600 & -2.43321800 & -2.78267600 \\
\hline $\mathrm{H}$ & 1.85980200 & 6.18323300 & 1.17533400 & $\mathrm{C}$ & -2.60226800 & -2.10010300 & -0.89693100 \\
\hline $\mathrm{H}$ & -0.05140400 & 7.77509400 & 1.17267900 & $\mathrm{O}$ & -1.69450800 & -2.25286300 & -1.75937100 \\
\hline $\mathrm{H}$ & -2.34063800 & 6.95417900 & 0.64870000 & $\mathrm{C}$ & -2.36052900 & -1.79564800 & 0.47930800 \\
\hline $\mathrm{H}$ & -2.71800100 & 4.56736500 & 0.14748600 & $\mathrm{H}$ & -3.16735100 & -1.31217200 & 1.02312200 \\
\hline $\mathrm{H}$ & -3.67474800 & 1.71958200 & -1.22958700 & & & & \\
\hline $\mathrm{H}$ & -5.82666400 & 1.25152800 & -0.14482800 & & & & \\
\hline $\mathrm{H}$ & -5.99949500 & 1.19891800 & 2.33665700 & 17 & & & \\
\hline $\mathrm{H}$ & -3.96741100 & 1.59244100 & 3.72142000 & $\mathrm{Ru}$ & -0.46205500 & 0.86353300 & 0.00944400 \\
\hline $\mathrm{H}$ & -1.79146000 & 2.01978400 & 2.63747000 & $\mathrm{P}$ & -2.65813800 & 0.19662200 & 0.60620900 \\
\hline $\mathrm{C}$ & 1.47310200 & 1.11256400 & 1.73648200 & $\mathrm{P}$ & 1.60713700 & 1.99530900 & 0.08527500 \\
\hline $\mathrm{O}$ & 1.85673100 & 1.35726000 & 2.81203100 & $\mathrm{~N}$ & -0.19984800 & 0.90169800 & 2.23097300 \\
\hline D & -0.13503900 & -0.65474900 & 0.68472800 & $\mathrm{C}$ & -2.47351700 & -0.00383700 & 2.45561200 \\
\hline $\mathrm{H}$ & 1.64520500 & 1.92636400 & -0.72606200 & $\mathrm{C}$ & -1.46501000 & 1.01988400 & 2.98800600 \\
\hline $\mathrm{H}$ & -0.39241200 & -0.86942100 & -1.76744100 & $\mathrm{C}$ & 1.46083900 & 2.73071800 & 1.79822200 \\
\hline $\mathrm{C}$ & -1.54371700 & -2.94377600 & 3.41912900 & $\mathrm{C}$ & 0.95198000 & 1.67119700 & 2.78201400 \\
\hline $\mathrm{H}$ & -2.58543300 & -3.19851000 & 3.18083300 & $\mathrm{C}$ & -3.88526800 & 2.66343700 & -0.08634300 \\
\hline $\mathrm{H}$ & -1.50247200 & -2.74713000 & 4.49881600 & $\mathrm{C}$ & -4.95421900 & 3.55968900 & -0.17591200 \\
\hline $\mathrm{C}$ & -1.14259100 & -1.66541600 & 2.64772300 & $\mathrm{C}$ & -6.22078800 & 3.18939700 & 0.27474900 \\
\hline $\mathrm{H}$ & -1.82691800 & -0.84395000 & 2.88443000 & $\mathrm{C}$ & -6.41961000 & 1.91426200 & 0.81194600 \\
\hline $\mathrm{H}$ & -0.14008000 & -1.35802800 & 2.96594700 & $\mathrm{C}$ & -5.35522100 & 1.01868400 & 0.89761300 \\
\hline $\mathrm{C}$ & -0.63040800 & -4.12346700 & 3.05350200 & $\mathrm{C}$ & -4.07146300 & 1.38517400 & 0.45596600 \\
\hline $\mathrm{H}$ & -0.96314600 & -5.03487500 & 3.56798700 & $\mathrm{C}$ & -3.53131800 & -1.56239500 & -1.36577400 \\
\hline $\mathrm{H}$ & 0.38845700 & -3.91432000 & 3.41210500 & $\mathrm{C}$ & -4.13802600 & -2.70811900 & -1.87803800 \\
\hline $\mathrm{C}$ & -0.58824000 & -4.35054500 & 1.53514200 & $\mathrm{C}$ & -4.62870200 & -3.68926200 & -1.01326900 \\
\hline $\mathrm{H}$ & -1.57372500 & -4.68223300 & 1.18233000 & $\mathrm{C}$ & -4.51041500 & -3.51714400 & 0.36590800 \\
\hline $\mathrm{H}$ & 0.12486400 & -5.14873300 & 1.29070700 & $\mathrm{C}$ & -3.91163900 & -2.36493300 & 0.88143600 \\
\hline $\mathrm{C}$ & -1.13849200 & -1.93814400 & 1.14791300 & $\mathrm{C}$ & -3.41816600 & -1.37344800 & 0.02116300 \\
\hline $\mathrm{C}$ & -0.17962700 & -3.06350300 & 0.77903300 & $\mathrm{C}$ & 0.84854600 & 4.04484700 & -1.70809700 \\
\hline $\mathrm{H}$ & 0.83513300 & -2.79216700 & 1.07992500 & $\mathrm{C}$ & 1.05135100 & 5.19034500 & -2.48063000 \\
\hline $\mathrm{H}$ & -0.18622100 & -3.23646500 & -0.29738400 & $\mathrm{C}$ & 2.31051900 & 5.78900200 & -2.53244700 \\
\hline $\mathrm{C}$ & -6.42626200 & -2.41194900 & -1.01385200 & $\mathrm{C}$ & 3.36982100 & 5.23507700 & -1.81013800 \\
\hline $\mathrm{C}$ & -5.12763500 & -2.32566100 & -0.51111900 & $\mathrm{C}$ & 3.17021700 & 4.08927500 & -1.04017600 \\
\hline
\end{tabular}




\begin{tabular}{|c|c|c|c|c|c|c|c|}
\hline $\mathrm{C}$ & 1.90425700 & 3.48346600 & -0.97440000 & $\mathrm{H}$ & 2.85478200 & -4.50614700 & -2.42889000 \\
\hline $\mathrm{C}$ & 4.27654200 & 1.35573800 & 0.94216700 & $\mathrm{H}$ & 2.78667400 & -4.32236700 & -4.17591100 \\
\hline $\mathrm{C}$ & 5.50772400 & 0.71561700 & 0.78648100 & $\mathrm{C}$ & 1.62939100 & -2.83770400 & -3.08840500 \\
\hline $\mathrm{C}$ & 5.71642900 & -0.14833500 & -0.28992900 & $\mathrm{H}$ & 2.46048400 & -2.12215200 & -3.02262700 \\
\hline $\mathrm{C}$ & 4.68327300 & -0.38514500 & -1.19922800 & $\mathrm{H}$ & 1.05904600 & -2.57115700 & -3.99115300 \\
\hline $\mathrm{C}$ & 3.44776500 & 0.24117600 & -1.03510200 & $\mathrm{C}$ & 1.04772600 & -5.30290600 & -3.30970200 \\
\hline $\mathrm{C}$ & 3.23535200 & 1.13456500 & 0.02688700 & $\mathrm{H}$ & 1.46440900 & -6.31673400 & -3.38213600 \\
\hline $\mathrm{H}$ & -3.43222900 & 0.09152800 & 2.97550000 & $\mathrm{H}$ & 0.45256400 & -5.14359000 & -4.22223100 \\
\hline $\mathrm{H}$ & -2.08222000 & -1.01284300 & 2.62383400 & $\mathrm{C}$ & 0.12848400 & -5.18300800 & -2.08379900 \\
\hline $\mathrm{H}$ & -1.84784900 & 2.04085200 & 2.87951100 & $\mathrm{H}$ & 0.69121900 & -5.44811900 & -1.17775400 \\
\hline $\mathrm{H}$ & -1.29054400 & 0.83915000 & 4.05971300 & $\mathrm{H}$ & -0.69844700 & -5.90224800 & -2.16001400 \\
\hline $\mathrm{H}$ & 2.39250700 & 3.18583700 & 2.15335900 & $\mathrm{C}$ & 0.70318700 & -2.68942100 & -1.85614000 \\
\hline $\mathrm{H}$ & 0.72367700 & 3.53397500 & 1.69258100 & $\mathrm{C}$ & -0.42270200 & -3.75685700 & -1.93799300 \\
\hline $\mathrm{H}$ & 1.75102500 & 0.95898200 & 3.01517600 & $\mathrm{H}$ & -1.06145500 & -3.53370200 & -2.80751500 \\
\hline $\mathrm{H}$ & 0.65851400 & 2.14830100 & 3.72518000 & $\mathrm{H}$ & -1.05725000 & -3.67771400 & -1.04901500 \\
\hline $\mathrm{H}$ & -2.89923300 & 2.95033300 & -0.43635000 & $\mathrm{C}$ & 4.01212300 & -2.66266000 & 3.00934500 \\
\hline $\mathrm{H}$ & -4.79327300 & 4.54668200 & -0.60161500 & $\mathrm{C}$ & 3.30176800 & -2.45847000 & 1.82596300 \\
\hline $\mathrm{H}$ & -7.05179300 & 3.88625900 & 0.20399200 & $\mathrm{C}$ & 1.90536500 & -2.29637600 & 1.83401900 \\
\hline $\mathrm{H}$ & -7.40567300 & 1.61519200 & 1.15764100 & $\mathrm{C}$ & 1.25212100 & -2.34188500 & 3.07933800 \\
\hline $\mathrm{H}$ & -5.52901100 & 0.02242900 & 1.29562100 & $\mathrm{C}$ & 1.95892300 & -2.55031100 & 4.26447900 \\
\hline $\mathrm{H}$ & -3.13350900 & -0.81776800 & -2.04956800 & $\mathrm{C}$ & 3.34639800 & -2.70968300 & 4.23677900 \\
\hline $\mathrm{H}$ & -4.21595100 & -2.83967500 & -2.95365800 & $\mathrm{H}$ & 5.09351700 & -2.77396400 & 2.97330300 \\
\hline $\mathrm{H}$ & -5.09395100 & -4.58610100 & -1.41311100 & $\mathrm{H}$ & 3.83297900 & -2.39676900 & 0.88179700 \\
\hline $\mathrm{H}$ & -4.88250000 & -4.27920500 & 1.04556400 & $\mathrm{H}$ & 0.17023200 & -2.24608900 & 3.10189300 \\
\hline $\mathrm{H}$ & -3.83307700 & -2.25122000 & 1.95814700 & $\mathrm{H}$ & 1.42448600 & -2.59906500 & 5.21095300 \\
\hline $\mathrm{H}$ & -0.13003100 & 3.57915200 & -1.67309100 & $\mathrm{H}$ & 3.90093900 & -2.86732300 & 5.15846200 \\
\hline $\mathrm{H}$ & 0.22394500 & 5.60844500 & -3.04782500 & $\mathrm{C}$ & 1.10863200 & -2.07361700 & 0.57733500 \\
\hline $\mathrm{H}$ & 2.46872000 & 6.67796500 & -3.13735100 & $\mathrm{O}$ & 0.05962400 & -1.26519400 & 0.71684000 \\
\hline $\mathrm{H}$ & 4.35563200 & 5.69097700 & -1.85047600 & $\mathrm{C}$ & 1.45628100 & -2.75020800 & -0.55137500 \\
\hline $\mathrm{H}$ & 4.00791000 & 3.65975400 & -0.49936900 & $\mathrm{H}$ & 2.29734500 & -3.43464900 & -0.48256600 \\
\hline $\mathrm{H}$ & 4.14086100 & 2.03095800 & 1.78175700 & & & & \\
\hline $\mathrm{H}$ & 6.30258200 & 0.89520100 & 1.50552300 & 18 & & & \\
\hline $\mathrm{H}$ & 6.67700200 & -0.64105500 & -0.41484200 & $\mathrm{Ru}$ & -1.07077600 & 0.34109600 & 0.06519500 \\
\hline $\mathrm{H}$ & 4.83182300 & -1.06891000 & -2.03031400 & $\mathrm{P}$ & 0.43081100 & 2.16880300 & -0.30268700 \\
\hline $\mathrm{H}$ & 2.64121200 & 0.03081300 & -1.73065100 & $\mathrm{P}$ & -2.71813600 & -1.27361400 & -0.48784700 \\
\hline $\mathrm{C}$ & -0.68178400 & 0.78777300 & -1.82874400 & $\mathrm{~N}$ & -0.14846200 & -0.36340000 & -1.82279900 \\
\hline $\mathrm{O}$ & -0.84463400 & 0.77401900 & -2.98270100 & $\mathrm{C}$ & 1.24867600 & 1.64221800 & -1.89349900 \\
\hline $\mathrm{H}$ & 0.21479200 & -1.70936200 & -1.91319100 & $\mathrm{C}$ & 0.27657300 & 0.76407400 & -2.68544100 \\
\hline $\mathrm{H}$ & -0.98467000 & 2.38053200 & -0.03958100 & $\mathrm{C}$ & -2.36410400 & -1.46851100 & -2.30929300 \\
\hline $\mathrm{H}$ & 0.05721100 & -0.09884700 & 2.16677100 & $\mathrm{C}$ & -0.85233000 & -1.44967000 & -2.55452100 \\
\hline $\mathrm{C}$ & 2.17967400 & -4.26417600 & -3.26198500 & $\mathrm{C}$ & -1.71584500 & 4.03892900 & -0.50252600 \\
\hline
\end{tabular}




\begin{tabular}{|c|c|c|c|c|c|c|c|}
\hline $\mathrm{C}$ & -2.28181400 & 5.27309700 & -0.83572500 & $\mathrm{H}$ & -4.19085800 & 1.17524700 & 0.26052500 \\
\hline $\mathrm{C}$ & -1.49946100 & 6.26926100 & -1.41820200 & $\mathrm{H}$ & -6.59037900 & 1.78385900 & 0.22496500 \\
\hline $\mathrm{C}$ & -0.14249000 & 6.03303100 & -1.65662200 & $\mathrm{H}$ & -8.28530300 & 0.12558400 & -0.52455900 \\
\hline $\mathrm{C}$ & 0.42310100 & 4.80595400 & -1.31561700 & $\mathrm{H}$ & -7.56203400 & -2.14924900 & -1.22520900 \\
\hline $\mathrm{C}$ & -0.35937900 & 3.78671800 & -0.74379400 & $\mathrm{H}$ & -5.17304500 & -2.76456100 & -1.16471200 \\
\hline $\mathrm{C}$ & 1.47781100 & 3.67562000 & 1.81431400 & $\mathrm{H}$ & -1.66274800 & -3.96226900 & -1.38236800 \\
\hline $\mathrm{C}$ & 2.46436400 & 4.14011500 & 2.68221500 & $\mathrm{H}$ & -1.64976900 & -6.16784500 & -0.30590400 \\
\hline $\mathrm{C}$ & 3.78135600 & 3.69271200 & 2.54968200 & $\mathrm{H}$ & -2.71113700 & -6.48847200 & 1.92066400 \\
\hline $\mathrm{C}$ & 4.09740200 & 2.76609100 & 1.55666600 & $\mathrm{H}$ & -3.79420800 & -4.55705400 & 3.05832400 \\
\hline $\mathrm{C}$ & 3.10918400 & 2.28737700 & 0.69234400 & $\mathrm{H}$ & -3.81366300 & -2.33701000 & 1.98680900 \\
\hline $\mathrm{C}$ & 1.79023800 & 2.75084800 & 0.80230300 & $\mathrm{C}$ & -1.84114600 & 1.00806500 & 1.63116500 \\
\hline $\mathrm{C}$ & -4.92641900 & 0.44918400 & -0.06628700 & $\mathrm{O}$ & -2.34247100 & 1.45107300 & 2.58203500 \\
\hline $\mathrm{C}$ & -6.28116600 & 0.79057700 & -0.08891300 & $\mathrm{H}$ & 4.40893600 & -0.98274600 & -2.25706900 \\
\hline $\mathrm{C}$ & -7.23176000 & -0.14018900 & -0.50760700 & $\mathrm{H}$ & -2.03309100 & 1.24412300 & -0.79836000 \\
\hline $\mathrm{C}$ & -6.82597900 & -1.41859500 & -0.90075200 & $\mathrm{H}$ & 0.77430300 & -0.82843600 & -1.48985200 \\
\hline $\mathrm{C}$ & -5.47545800 & -1.76262500 & -0.87295400 & $\mathrm{C}$ & 7.30188100 & -1.74483300 & -0.44911500 \\
\hline $\mathrm{C}$ & -4.50878700 & -0.82956600 & -0.45856600 & $\mathrm{H}$ & 6.95205300 & -1.67827800 & 0.59096900 \\
\hline $\mathrm{C}$ & -2.13324100 & -4.07406400 & -0.41126500 & $\mathrm{H}$ & 8.31535300 & -1.32028600 & -0.46027200 \\
\hline $\mathrm{C}$ & -2.12492200 & -5.33124100 & 0.19881500 & $\mathrm{C}$ & 6.37650500 & -0.92070900 & -1.36192300 \\
\hline $\mathrm{C}$ & -2.72012500 & -5.51086800 & 1.44692700 & $\mathrm{H}$ & 6.33635600 & 0.12132700 & -1.01455000 \\
\hline $\mathrm{C}$ & -3.32614300 & -4.42610900 & 2.08649400 & $\mathrm{H}$ & 6.81481900 & -0.89813000 & -2.37154600 \\
\hline $\mathrm{C}$ & -3.33243900 & -3.17061100 & 1.48117500 & $\mathrm{C}$ & 7.34014400 & -3.22270000 & -0.87068200 \\
\hline $\mathrm{C}$ & -2.73910000 & -2.97784200 & 0.22037300 & $\mathrm{H}$ & 7.96637600 & -3.79958400 & -0.17633100 \\
\hline $\mathrm{H}$ & 1.57750400 & 2.50561300 & -2.47966100 & $\mathrm{H}$ & 7.81679800 & -3.30167800 & -1.86020300 \\
\hline $\mathrm{H}$ & 2.12305100 & 1.03283100 & -1.64601800 & $\mathrm{C}$ & 5.92521000 & -3.81913500 & -0.94601800 \\
\hline $\mathrm{H}$ & -0.59732400 & 1.33500800 & -3.02089600 & $\mathrm{H}$ & 5.48320900 & -3.83700900 & 0.06009300 \\
\hline $\mathrm{H}$ & 0.79362900 & 0.37466000 & -3.57411600 & $\mathrm{H}$ & 5.97305200 & -4.86244100 & -1.28718700 \\
\hline $\mathrm{H}$ & -2.82838500 & -2.36991900 & -2.72684100 & $\mathrm{C}$ & 4.94730300 & -1.50227100 & -1.45129900 \\
\hline $\mathrm{H}$ & -2.83906800 & -0.60250700 & -2.78429100 & $\mathrm{C}$ & 5.02298000 & -2.99487400 & -1.87524700 \\
\hline $\mathrm{H}$ & -0.39428000 & -2.38770800 & -2.22329600 & $\mathrm{H}$ & 5.41995100 & -3.05510500 & -2.90210100 \\
\hline $\mathrm{H}$ & -0.65406800 & -1.36781700 & -3.63129400 & $\mathrm{H}$ & 4.00621900 & -3.40029000 & -1.89880800 \\
\hline $\mathrm{H}$ & -2.33054900 & 3.26818300 & -0.05204500 & $\mathrm{C}$ & 1.83973500 & -0.47443100 & 3.45498400 \\
\hline $\mathrm{H}$ & -3.33546500 & 5.45160000 & -0.63820500 & $\mathrm{C}$ & 2.58287900 & -0.59624200 & 2.28074100 \\
\hline $\mathrm{H}$ & -1.94032800 & 7.22714500 & -1.68097300 & $\mathrm{C}$ & 2.03490200 & -1.17138000 & 1.12166000 \\
\hline $\mathrm{H}$ & 0.47647400 & 6.80684700 & -2.10276800 & $\mathrm{C}$ & 0.69420200 & -1.61766800 & 1.19279600 \\
\hline $\mathrm{H}$ & 1.48519300 & 4.64695900 & -1.48216700 & $\mathrm{C}$ & -0.04796100 & -1.50829400 & 2.37990000 \\
\hline $\mathrm{H}$ & 0.46216000 & 4.04790600 & 1.91712400 & $\mathrm{C}$ & 0.51958100 & -0.92607600 & 3.51603700 \\
\hline $\mathrm{H}$ & 2.20480100 & 4.85663400 & 3.45697700 & $\mathrm{H}$ & 2.29761100 & -0.01949600 & 4.33028200 \\
\hline $\mathrm{H}$ & 4.55336900 & 4.06126000 & 3.21997200 & $\mathrm{H}$ & 3.60094000 & -0.22402500 & 2.26133500 \\
\hline $\mathrm{H}$ & 5.11548200 & 2.40055500 & 1.45154900 & $\mathrm{H}$ & 0.30723600 & -2.18184700 & 0.35093300 \\
\hline $\mathrm{H}$ & 3.38107600 & 1.52780400 & -0.03371700 & $\mathrm{H}$ & -1.04881400 & -1.92618500 & 2.42469500 \\
\hline
\end{tabular}




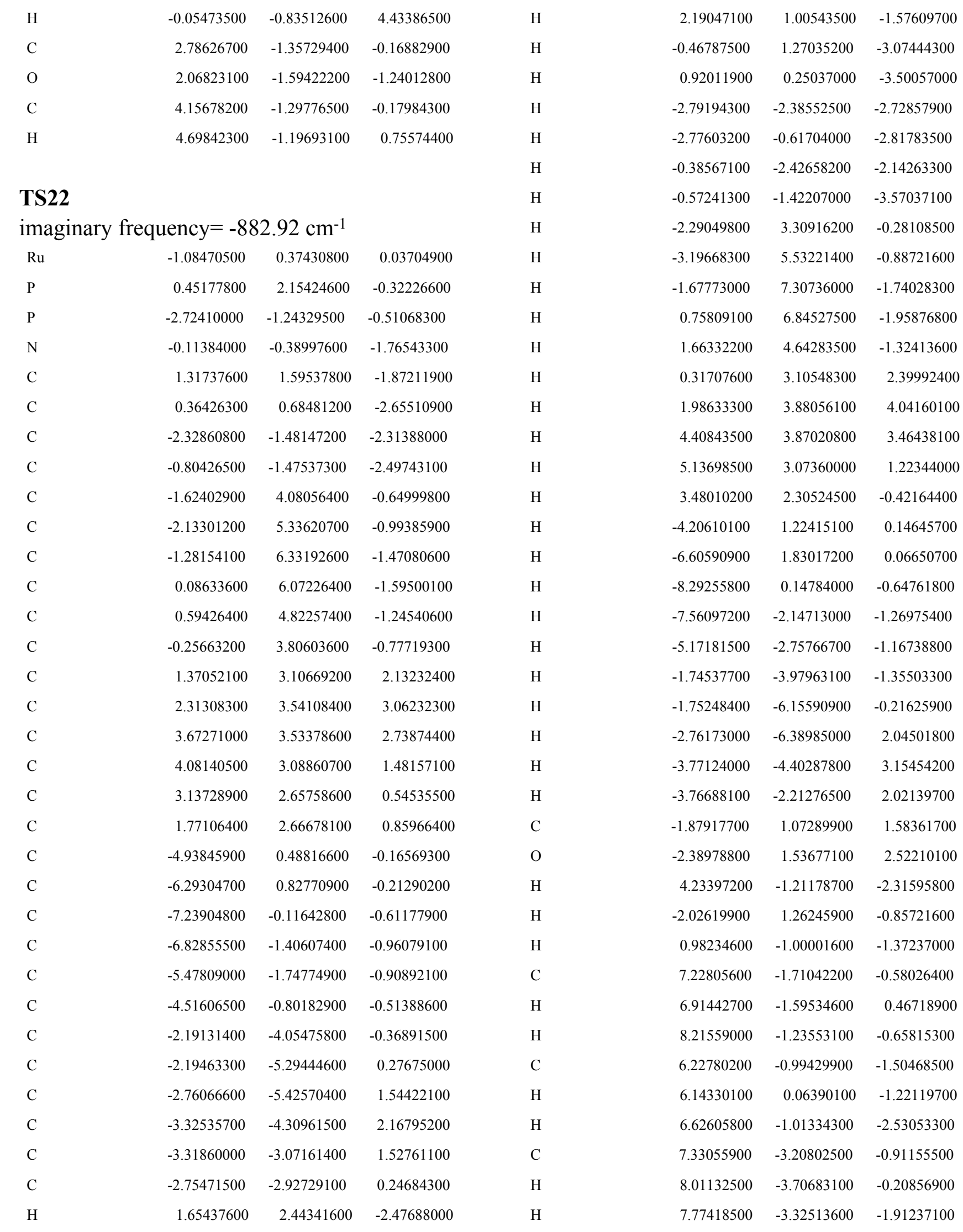




\begin{tabular}{|c|c|c|c|c|c|c|c|}
\hline $\mathrm{C}$ & 5.94838200 & -3.88066000 & -0.89343100 & $\mathrm{C}$ & 0.61230200 & -5.44243600 & 1.85575200 \\
\hline $\mathrm{H}$ & 5.54664800 & -3.86143000 & 0.12951900 & $\mathrm{C}$ & 0.63526800 & -4.48143000 & 0.84254500 \\
\hline $\mathrm{H}$ & 6.03945500 & -4.93866400 & -1.17412500 & $\mathrm{C}$ & 1.50580100 & -3.38351500 & 0.92109200 \\
\hline $\mathrm{C}$ & 4.82859300 & -1.65334000 & -1.50533200 & $\mathrm{C}$ & 3.92734500 & 3.26456400 & 0.15360800 \\
\hline $\mathrm{C}$ & 4.96906900 & -3.16456400 & -1.83476400 & $\mathrm{C}$ & 5.01493100 & 4.14245000 & 0.14629300 \\
\hline $\mathrm{H}$ & 5.33112100 & -3.26852700 & -2.87031100 & $\mathrm{C}$ & 4.83953200 & 5.47266700 & -0.23498800 \\
\hline $\mathrm{H}$ & 3.97772800 & -3.62860700 & -1.79442900 & $\mathrm{C}$ & 3.57096700 & 5.92905800 & -0.60529100 \\
\hline $\mathrm{C}$ & 1.89353900 & -0.34200000 & 3.42770200 & $\mathrm{C}$ & 2.48394600 & 5.05637800 & -0.59245900 \\
\hline $\mathrm{C}$ & 2.58536500 & -0.50201700 & 2.22795600 & $\mathrm{C}$ & 2.65223200 & 3.71199700 & -0.21693000 \\
\hline $\mathrm{C}$ & 1.99763900 & -1.15247600 & 1.12959900 & $\mathrm{C}$ & -1.40153400 & 3.31628500 & 0.46777100 \\
\hline $\mathrm{C}$ & 0.67250400 & -1.61902000 & 1.27734000 & $\mathrm{C}$ & -2.35738900 & 3.92276900 & 1.28572000 \\
\hline $\mathrm{C}$ & -0.02124800 & -1.45570100 & 2.48453000 & $\mathrm{C}$ & -1.96486600 & 4.63504600 & 2.41988500 \\
\hline $\mathrm{C}$ & 0.58648600 & -0.81447100 & 3.56627800 & $\mathrm{C}$ & -0.60849500 & 4.73458900 & 2.73738100 \\
\hline $\mathrm{H}$ & 2.37720800 & 0.16783900 & 4.25711700 & $\mathrm{C}$ & 0.34970200 & 4.13218200 & 1.92173700 \\
\hline $\mathrm{H}$ & 3.58741800 & -0.09759500 & 2.13399100 & $\mathrm{C}$ & -0.03484000 & 3.42168900 & 0.77228000 \\
\hline $\mathrm{H}$ & 0.24113600 & -2.21098200 & 0.47766400 & $\mathrm{H}$ & 0.07519600 & -3.21981300 & -2.01033900 \\
\hline $\mathrm{H}$ & -1.02017400 & -1.86884200 & 2.58439900 & $\mathrm{H}$ & -0.78261200 & -2.34682400 & -0.71666400 \\
\hline $\mathrm{H}$ & 0.04950600 & -0.68720400 & 4.50223500 & $\mathrm{H}$ & 0.61430300 & -1.16585600 & -3.17772000 \\
\hline $\mathrm{C}$ & 2.71796100 & -1.39384000 & -0.15659100 & $\mathrm{H}$ & -1.08899100 & -1.03706600 & -2.73303000 \\
\hline $\mathrm{O}$ & 1.96680800 & -1.65190700 & -1.23287000 & $\mathrm{H}$ & 0.03582900 & 3.51580200 & -2.20046900 \\
\hline $\mathrm{C}$ & 4.07645100 & -1.40607200 & -0.21921600 & $\mathrm{H}$ & 1.56451500 & 2.72220000 & -2.59107900 \\
\hline \multirow[t]{2}{*}{$\mathrm{H}$} & 4.64420000 & -1.29678800 & 0.69959600 & $\mathrm{H}$ & -1.18328200 & 1.50872500 & -2.45164400 \\
\hline & & & & $\mathrm{H}$ & 0.18683700 & 1.11060300 & -3.47646800 \\
\hline 19 & & & & $\mathrm{H}$ & 3.29754600 & -0.74093400 & -2.32769200 \\
\hline $\mathrm{Ru}$ & 1.49911100 & 0.23436900 & 0.03169900 & $\mathrm{H}$ & 5.01398400 & -1.51227700 & -3.94161600 \\
\hline $\mathrm{P}$ & 1.59707300 & -2.09231300 & -0.38538400 & $\mathrm{H}$ & 5.67051100 & -3.91149000 & -4.01076100 \\
\hline $\mathrm{P}$ & 1.22831700 & 2.54183600 & -0.24376300 & $\mathrm{H}$ & 4.58432300 & -5.53114300 & -2.46658100 \\
\hline $\mathrm{N}$ & 0.21145100 & 0.17458800 & -1.57657100 & $\mathrm{H}$ & 2.85750300 & -4.76811400 & -0.87949000 \\
\hline $\mathrm{C}$ & 0.05991700 & -2.29909700 & -1.41615300 & $\mathrm{H}$ & 3.01585700 & -2.41415900 & 2.12533500 \\
\hline $\mathrm{C}$ & -0.06896200 & -1.06538400 & -2.31483100 & $\mathrm{H}$ & 2.98660300 & -4.12398700 & 3.90464700 \\
\hline $\mathrm{C}$ & 0.63940800 & 2.62043000 & -2.01286600 & $\mathrm{H}$ & 1.44116100 & -6.06844000 & 3.74561200 \\
\hline $\mathrm{C}$ & -0.09611600 & 1.33426000 & -2.43376600 & $\mathrm{H}$ & -0.06925900 & -6.28578600 & 1.78167200 \\
\hline $\mathrm{C}$ & 3.56801600 & -1.79152600 & -2.37237400 & $\mathrm{H}$ & -0.03119100 & -4.59277200 & -0.00709600 \\
\hline $\mathrm{C}$ & 4.53957500 & -2.22770000 & -3.27488800 & $\mathrm{H}$ & 4.06281900 & 2.22765400 & 0.44429900 \\
\hline $\mathrm{C}$ & 4.90830100 & -3.57353000 & -3.31356500 & $\mathrm{H}$ & 5.99787700 & 3.78323100 & 0.43910100 \\
\hline $\mathrm{C}$ & 4.29799800 & -4.48275000 & -2.44740100 & $\mathrm{H}$ & 5.68594600 & 6.15439900 & -0.24210500 \\
\hline $\mathrm{C}$ & 3.32060300 & -4.05034600 & -1.54959300 & $\mathrm{H}$ & 3.42855100 & 6.96528600 & -0.90053500 \\
\hline $\mathrm{C}$ & 2.94256300 & -2.69914500 & -1.50276900 & $\mathrm{H}$ & 1.49871400 & 5.42487900 & -0.86598700 \\
\hline $\mathrm{C}$ & 2.34935300 & -3.26673500 & 2.03798400 & $\mathrm{H}$ & -1.73758200 & 2.74197600 & -0.38910400 \\
\hline $\mathrm{C}$ & 2.32873400 & -4.22997900 & 3.04632600 & $\mathrm{H}$ & -3.41052500 & 3.83006500 & 1.03429500 \\
\hline $\mathrm{C}$ & 1.45991700 & -5.32019200 & 2.95762900 & $\mathrm{H}$ & -2.71056400 & 5.10513500 & 3.05538800 \\
\hline
\end{tabular}




\begin{tabular}{|c|c|c|c|c|c|c|c|}
\hline $\mathrm{H}$ & -0.29299200 & 5.28360800 & 3.62071700 & $\mathrm{H}$ & -4.29752200 & 0.31401700 & -1.59666400 \\
\hline $\mathrm{H}$ & 1.40135900 & 4.22420800 & 2.17712700 & $\mathrm{H}$ & -4.85252400 & 1.75200900 & -0.74510800 \\
\hline $\mathrm{C}$ & 2.11617800 & 0.25436500 & 1.80050300 & $\mathrm{C}$ & -2.73336100 & 1.33342700 & -0.50578400 \\
\hline $\mathrm{O}$ & 2.44212700 & 0.26255300 & 2.92129700 & $\mathrm{H}$ & -2.43347400 & 1.93314900 & -1.37647600 \\
\hline $\mathrm{H}$ & -4.38947800 & 0.16295300 & -2.38768800 & $\mathrm{H}$ & -2.65067300 & 1.99564500 & 0.37055100 \\
\hline $\mathrm{H}$ & 3.02831600 & 0.36748200 & -0.33674700 & $\mathrm{C}$ & -4.63349700 & -0.00957100 & 0.51552700 \\
\hline $\mathrm{H}$ & -1.60324900 & 0.18963800 & -0.65801900 & $\mathrm{H}$ & -5.66144200 & -0.36203300 & 0.36055700 \\
\hline $\mathrm{C}$ & -7.53424700 & -1.18023300 & -1.66183100 & $\mathrm{H}$ & -4.64418300 & 0.59024200 & 1.43813000 \\
\hline $\mathrm{H}$ & -7.35552800 & -1.70678000 & -0.71332400 & $\mathrm{C}$ & -3.68079500 & -1.20034300 & 0.69801100 \\
\hline $\mathrm{H}$ & -8.21233100 & -1.82002500 & -2.24190900 & $\mathrm{H}$ & -3.76026200 & -1.86536700 & -0.17445200 \\
\hline $\mathrm{C}$ & -6.20778700 & -1.00502100 & -2.42107900 & $\mathrm{H}$ & -3.97831500 & -1.79600400 & 1.57065400 \\
\hline $\mathrm{H}$ & -5.73159900 & -1.98062400 & -2.58430900 & $\mathrm{C}$ & -2.22204700 & -0.74114800 & 0.84831800 \\
\hline $\mathrm{H}$ & -6.42643000 & -0.59245300 & -3.41723000 & $\mathrm{H}$ & -2.11998200 & -0.16497300 & 1.78215200 \\
\hline $\mathrm{C}$ & -8.20081600 & 0.17332700 & -1.36861600 & $\mathrm{H}$ & -1.56038400 & -1.60938700 & 0.93272500 \\
\hline $\mathrm{H}$ & -9.11871100 & 0.02671400 & -0.78429500 & $\mathrm{C}$ & 3.95564000 & 1.42021900 & 0.49193600 \\
\hline $\mathrm{H}$ & -8.50582600 & 0.63755100 & -2.31886900 & $\mathrm{C}$ & 2.60160800 & 1.16217600 & 0.27588300 \\
\hline $\mathrm{C}$ & -7.24124500 & 1.12177200 & -0.63229600 & $\mathrm{C}$ & 2.17564800 & -0.11653600 & -0.11594000 \\
\hline $\mathrm{H}$ & -7.02255500 & 0.71771800 & 0.36633100 & $\mathrm{C}$ & 3.13247800 & -1.13033300 & -0.28719700 \\
\hline $\mathrm{H}$ & -7.71994000 & 2.09735300 & -0.47514600 & $\mathrm{C}$ & 4.48272000 & -0.87372200 & -0.06919700 \\
\hline $\mathrm{C}$ & -5.22074300 & -0.05087700 & -1.70498400 & $\mathrm{C}$ & 4.89742600 & 0.40359800 & 0.32117800 \\
\hline $\mathrm{C}$ & -5.92852300 & 1.29944500 & -1.41009200 & $\mathrm{H}$ & 4.27483400 & 2.41405100 & 0.79370200 \\
\hline $\mathrm{H}$ & -6.14763700 & 1.79456400 & -2.36879100 & $\mathrm{H}$ & 1.88207200 & 1.96327800 & 0.41178600 \\
\hline $\mathrm{H}$ & -5.24194500 & 1.95663600 & -0.86320300 & $\mathrm{H}$ & 2.78748500 & -2.11269600 & -0.59270800 \\
\hline $\mathrm{C}$ & -2.62241300 & -3.02395200 & 2.67659900 & $\mathrm{H}$ & 5.21415100 & -1.66618000 & -0.20288800 \\
\hline $\mathrm{C}$ & -3.15259100 & -2.43355000 & 1.53068200 & $\mathrm{H}$ & 5.95169300 & 0.60549800 & 0.49137200 \\
\hline $\mathrm{C}$ & -2.78547100 & -1.12801900 & 1.15811100 & $\mathrm{C}$ & 0.73455200 & -0.45837500 & -0.37027500 \\
\hline $\mathrm{C}$ & -1.85563500 & -0.44094400 & 1.95907800 & $\mathrm{O}$ & 0.42780300 & -1.58046600 & -0.74822000 \\
\hline $\mathrm{C}$ & -1.31949000 & -1.03716000 & 3.10125200 & $\mathrm{C}$ & -0.32330300 & 0.62544300 & -0.17105100 \\
\hline $\mathrm{C}$ & -1.70179800 & -2.32941700 & 3.46588900 & $\mathrm{H}$ & -0.10924300 & 1.42460400 & -0.89811900 \\
\hline $\mathrm{H}$ & -2.91634100 & -4.03535500 & 2.94564000 & $\mathrm{H}$ & -0.18385900 & 1.08699600 & 0.81719300 \\
\hline $\mathrm{H}$ & -3.84475400 & -2.98808300 & 0.90345500 & $\mathrm{C}$ & -1.77128700 & 0.14211200 & -0.33219500 \\
\hline $\mathrm{H}$ & -1.57255100 & 0.57428100 & 1.69920200 & $\mathrm{H}$ & -1.81517800 & -0.47199700 & -1.24238000 \\
\hline $\mathrm{H}$ & -0.59932500 & -0.48980900 & 3.70300900 & & & & \\
\hline $\mathrm{H}$ & -1.27696200 & -2.79644400 & 4.35001800 & \multicolumn{4}{|c|}{ TS23 } \\
\hline $\mathrm{C}$ & -3.36239600 & -0.48369100 & -0.04751200 & \multicolumn{4}{|c|}{ imaginary frequency $=-503.43 \mathrm{~cm}^{-1}$} \\
\hline $\mathrm{O}$ & -2.56546400 & 0.41207800 & -0.71917500 & $\mathrm{Ru}$ & -0.01578900 & 0.70799800 & -0.15645600 \\
\hline $\mathrm{C}$ & -4.62834400 & -0.67782200 & -0.46579000 & $\mathrm{P}$ & 2.26091100 & 0.93924000 & -0.80975700 \\
\hline $\mathrm{H}$ & -5.26411200 & -1.30158800 & 0.15526600 & $\mathrm{P}$ & -2.30299700 & 1.21482800 & -0.18417500 \\
\hline
\end{tabular}

H

C

O

C

$\mathrm{H}$

pro

C $\begin{array}{lll}-5.26411200 & -1.30158800 & 0.15526600\end{array}$

$\begin{array}{lll}-4.19338500 & 0.87853100 & -0.65825900\end{array}$

\section{TS23}

imaginary frequency $=-503.43 \mathrm{~cm}^{-1}$

$\begin{array}{lrrr}\mathrm{Ru} & -0.01578900 & 0.70799800 & -0.15645600 \\ \mathrm{P} & 2.26091100 & 0.93924000 & -0.80975700 \\ \mathrm{P} & -2.30299700 & 1.21482800 & -0.18417500 \\ \mathrm{~N} & -0.29484700 & 0.48856700 & -2.35508800 \\ \mathrm{C} & 2.10229100 & 0.92638000 & -2.68077100 \\ \mathrm{C} & 0.68297100 & 1.31710900 & -3.10217400\end{array}$




\begin{tabular}{|c|c|c|c|c|c|c|c|}
\hline $\mathrm{C}$ & -2.51389300 & 1.60844000 & -1.99885200 & $\mathrm{H}$ & 6.62492200 & -2.66349300 & 0.23553000 \\
\hline $\mathrm{C}$ & -1.70161500 & 0.61161500 & -2.82894300 & $\mathrm{H}$ & 6.45907400 & -0.50474000 & 1.46686100 \\
\hline $\mathrm{C}$ & 4.19686000 & 2.98353500 & -1.18190500 & $\mathrm{H}$ & 4.60969500 & 1.04980300 & 0.99878700 \\
\hline $\mathrm{C}$ & 4.80808100 & 4.20896100 & -0.91897900 & $\mathrm{H}$ & -0.96671300 & 3.48172900 & 1.14486700 \\
\hline $\mathrm{C}$ & 4.30559500 & 5.04031800 & 0.08555100 & $\mathrm{H}$ & -1.78924600 & 5.57714500 & 2.18475300 \\
\hline $\mathrm{C}$ & 3.19246400 & 4.63683500 & 0.82283800 & $\mathrm{H}$ & -4.22865900 & 6.06153500 & 2.24992600 \\
\hline $\mathrm{C}$ & 2.57258300 & 3.41449000 & 0.54906200 & $\mathrm{H}$ & -5.83523100 & 4.42424700 & 1.28469100 \\
\hline $\mathrm{C}$ & 3.06263300 & 2.57422100 & -0.45911900 & $\mathrm{H}$ & -5.01765600 & 2.32668900 & 0.28446400 \\
\hline $\mathrm{C}$ & 3.75063200 & -1.45477500 & -1.14474600 & $\mathrm{H}$ & -4.77169600 & 0.04152700 & -1.56601700 \\
\hline $\mathrm{C}$ & 4.81475700 & -2.32184800 & -0.89055200 & $\mathrm{H}$ & -6.54936900 & -1.43630500 & -0.73243000 \\
\hline $\mathrm{C}$ & 5.79816700 & -1.98502700 & 0.04157700 & $\mathrm{H}$ & -6.47928900 & -2.29673400 & 1.59948000 \\
\hline $\mathrm{C}$ & 5.70665800 & -0.77397600 & 0.73007900 & $\mathrm{H}$ & -4.56622100 & -1.69816300 & 3.07695100 \\
\hline $\mathrm{C}$ & 4.65416600 & 0.10448200 & 0.46737100 & $\mathrm{H}$ & -2.75763000 & -0.24199200 & 2.23464500 \\
\hline $\mathrm{C}$ & 3.67237200 & -0.21732100 & -0.48481000 & $\mathrm{C}$ & 0.21506300 & 0.84607600 & 1.67797600 \\
\hline $\mathrm{C}$ & -2.03023100 & 3.68879000 & 1.18080700 & $\mathrm{O}$ & 0.33882300 & 0.95608500 & 2.83310500 \\
\hline $\mathrm{C}$ & -2.49777000 & 4.87097000 & 1.75971200 & $\mathrm{H}$ & -0.05465400 & -1.11473800 & -0.20875400 \\
\hline $\mathrm{C}$ & -3.86551600 & 5.14241000 & 1.79751300 & $\mathrm{H}$ & 0.04551100 & 2.33875200 & -0.36886900 \\
\hline $\mathrm{C}$ & -4.76739800 & 4.22333000 & 1.25609500 & $\mathrm{H}$ & -0.00314000 & -0.50244700 & -2.48042400 \\
\hline $\mathrm{C}$ & -4.30241700 & 3.03982000 & 0.68331000 & $\mathrm{C}$ & 3.21134200 & -3.98564600 & 2.62040600 \\
\hline $\mathrm{C}$ & -2.92593200 & 2.75997700 & 0.63258900 & $\mathrm{H}$ & 3.87898200 & -3.26579800 & 2.12863400 \\
\hline $\mathrm{C}$ & -4.71168700 & -0.32999900 & -0.54756200 & $\mathrm{H}$ & 3.84331800 & -4.79665300 & 3.00624100 \\
\hline $\mathrm{C}$ & -5.72590300 & -1.16802900 & -0.07583600 & $\mathrm{C}$ & 2.22315000 & -4.54834800 & 1.58007800 \\
\hline $\mathrm{C}$ & -5.68458200 & -1.65359400 & 1.23073800 & $\mathrm{H}$ & 2.76535200 & -4.99708600 & 0.73964000 \\
\hline $\mathrm{C}$ & -4.61165800 & -1.31650900 & 2.06034700 & $\mathrm{H}$ & 1.65116900 & -5.36198000 & 2.05703600 \\
\hline $\mathrm{C}$ & -3.59341800 & -0.49196600 & 1.58582700 & $\mathrm{C}$ & 2.46669200 & -3.28882800 & 3.76697200 \\
\hline $\mathrm{C}$ & -3.63954000 & 0.02789000 & 0.28087100 & $\mathrm{H}$ & 3.18186500 & -2.85408500 & 4.47749100 \\
\hline $\mathrm{H}$ & 2.83833200 & 1.58925100 & -3.14509200 & $\mathrm{H}$ & 1.88081000 & -4.03349000 & 4.32778600 \\
\hline $\mathrm{H}$ & 2.30599000 & -0.09433100 & -3.01481900 & $\mathrm{C}$ & 1.52934600 & -2.20218400 & 3.22568500 \\
\hline $\mathrm{H}$ & 0.48542800 & 2.37267500 & -2.89351200 & $\mathrm{H}$ & 2.12672300 & -1.41489400 & 2.74822500 \\
\hline $\mathrm{H}$ & 0.56686500 & 1.15432100 & -4.18521400 & $\mathrm{H}$ & 0.97629500 & -1.72284900 & 4.04226800 \\
\hline $\mathrm{H}$ & -3.56154900 & 1.62732900 & -2.31965500 & $\mathrm{C}$ & 1.23919100 & -3.50430900 & 1.07917900 \\
\hline $\mathrm{H}$ & -2.12027200 & 2.62379700 & -2.11646700 & $\mathrm{C}$ & 0.52971200 & -2.76353600 & 2.18988800 \\
\hline $\mathrm{H}$ & -2.15112300 & -0.38277800 & -2.75298400 & $\mathrm{H}$ & -0.14332300 & -3.46729400 & 2.70885300 \\
\hline $\mathrm{H}$ & -1.71759900 & 0.89831300 & -3.89032900 & $\mathrm{H}$ & -0.08620800 & -1.95681100 & 1.78877000 \\
\hline $\mathrm{H}$ & 4.61952400 & 2.33581900 & -1.94539600 & $\mathrm{C}$ & -3.27562700 & -3.98027000 & -0.33119900 \\
\hline $\mathrm{H}$ & 5.67943500 & 4.51174000 & -1.49377900 & $\mathrm{C}$ & -2.00614900 & -3.43577900 & -0.14386000 \\
\hline $\mathrm{H}$ & 4.78307100 & 5.99428100 & 0.29340800 & $\mathrm{C}$ & -1.25467100 & -2.98188300 & -1.23623400 \\
\hline $\mathrm{H}$ & 2.79998900 & 5.27332000 & 1.61154400 & $\mathrm{C}$ & -1.80622000 & -3.09996800 & -2.52112600 \\
\hline $\mathrm{H}$ & 1.69960700 & 3.11035500 & 1.11500100 & $\mathrm{C}$ & -3.07807400 & -3.64340300 & -2.71065300 \\
\hline $\mathrm{H}$ & 2.96200200 & -1.76116300 & -1.82564200 & $\mathrm{C}$ & -3.81851400 & -4.08712300 & -1.61444000 \\
\hline $\mathrm{H}$ & 4.86757400 & -3.27024200 & -1.41927800 & $\mathrm{H}$ & -3.84447000 & -4.32166800 & 0.52920600 \\
\hline
\end{tabular}




\begin{tabular}{|c|c|c|c|c|c|c|c|}
\hline $\mathrm{H}$ & -1.60056900 & -3.36592000 & 0.85781600 & $\mathrm{C}$ & 1.16107800 & 2.85625400 & -0.57696700 \\
\hline $\mathrm{H}$ & -1.20072000 & -2.79158600 & -3.36696600 & $\mathrm{H}$ & -2.01852000 & -2.70175000 & 2.62086700 \\
\hline $\mathrm{H}$ & -3.48094900 & -3.73773800 & -3.71652300 & $\mathrm{H}$ & -0.49714600 & -2.46362300 & 1.74053300 \\
\hline $\mathrm{H}$ & -4.80526600 & -4.52058200 & -1.75677900 & $\mathrm{H}$ & -2.13854800 & -0.35645500 & 3.22844100 \\
\hline $\mathrm{C}$ & 0.19132400 & -2.49575500 & -1.14027900 & $\mathrm{H}$ & -0.45572700 & -0.82873400 & 3.48067100 \\
\hline $\mathrm{O}$ & 0.72012700 & -2.08591500 & -2.22313800 & $\mathrm{H}$ & -0.05979400 & 3.71663100 & 2.13343800 \\
\hline $\mathrm{C}$ & 1.09472400 & -3.31907300 & -0.24358700 & $\mathrm{H}$ & -1.71324200 & 3.11508700 & 1.96957500 \\
\hline \multirow[t]{2}{*}{$\mathrm{H}$} & 1.73858300 & -3.92033000 & -0.88806900 & $\mathrm{H}$ & 0.78705100 & 1.50731500 & 2.65376600 \\
\hline & & & & $\mathrm{H}$ & -0.75070300 & 1.59441700 & 3.50317600 \\
\hline 20 & & & & $\mathrm{H}$ & -3.88556100 & -3.73493500 & 1.85376900 \\
\hline $\mathrm{Ru}$ & -1.47049200 & 0.44694400 & -0.18180300 & $\mathrm{H}$ & -6.28863300 & -3.99143600 & 2.33464900 \\
\hline $\mathrm{P}$ & -2.32190500 & -1.68661100 & 0.36424800 & $\mathrm{H}$ & -7.92376400 & -2.33137000 & 1.46272300 \\
\hline $\mathrm{P}$ & -0.59446000 & 2.61692600 & -0.05780900 & $\mathrm{H}$ & -7.12319700 & -0.40556800 & 0.10729500 \\
\hline $\mathrm{N}$ & -0.78114100 & 0.37406200 & 1.76360100 & $\mathrm{H}$ & -4.70050200 & -0.13232600 & -0.35210300 \\
\hline $\mathrm{C}$ & -1.45894300 & -2.00512400 & 1.98778100 & $\mathrm{H}$ & 0.24236300 & -2.77376200 & -0.47391900 \\
\hline $\mathrm{C}$ & -1.21977500 & -0.67078900 & 2.70115300 & $\mathrm{H}$ & 0.90909400 & -4.64633200 & -1.92800700 \\
\hline $\mathrm{C}$ & -0.66143800 & 2.87078700 & 1.77982400 & $\mathrm{H}$ & -0.81639300 & -6.07773600 & -3.01230700 \\
\hline $\mathrm{C}$ & -0.30328000 & 1.55989500 & 2.49700600 & $\mathrm{H}$ & -3.22761100 & -5.61361900 & -2.61335100 \\
\hline $\mathrm{C}$ & -4.58500700 & -2.99229600 & 1.47958700 & $\mathrm{H}$ & -3.90787600 & -3.75888200 & -1.13781800 \\
\hline $\mathrm{C}$ & -5.94549400 & -3.14499500 & 1.74554900 & $\mathrm{H}$ & -3.19176200 & 2.99610100 & -1.32488900 \\
\hline $\mathrm{C}$ & -6.86368500 & -2.21315200 & 1.25422600 & $\mathrm{H}$ & -4.39545100 & 4.98055800 & -2.20718600 \\
\hline $\mathrm{C}$ & -6.41444600 & -1.13273900 & 0.49456100 & $\mathrm{H}$ & -3.31534500 & 7.21997500 & -2.14376300 \\
\hline $\mathrm{C}$ & -5.05117800 & -0.97764000 & 0.23131700 & $\mathrm{H}$ & -1.02632800 & 7.46146900 & -1.20172500 \\
\hline $\mathrm{C}$ & -4.11953000 & -1.90265600 & 0.72290800 & $\mathrm{H}$ & 0.17455300 & 5.48613000 & -0.33720600 \\
\hline $\mathrm{C}$ & -0.52431300 & -3.39924000 & -0.92585000 & $\mathrm{H}$ & 1.88370900 & 3.83049500 & 1.21776400 \\
\hline $\mathrm{C}$ & -0.14694000 & -4.45575700 & -1.75406600 & $\mathrm{H}$ & 4.17762800 & 4.13512200 & 0.38240900 \\
\hline $\mathrm{C}$ & -1.11491200 & -5.25869800 & -2.36310200 & $\mathrm{H}$ & 4.80142400 & 3.34362600 & -1.89188600 \\
\hline $\mathrm{C}$ & -2.46682300 & -4.99907500 & -2.13890700 & $\mathrm{H}$ & 3.09585300 & 2.22297600 & -3.31848800 \\
\hline $\mathrm{C}$ & -2.85212400 & -3.94764500 & -1.30335000 & $\mathrm{H}$ & 0.79689100 & 1.89307200 & -2.47693000 \\
\hline $\mathrm{C}$ & -1.88627100 & -3.14318400 & -0.68218900 & $\mathrm{C}$ & -1.61782300 & 0.30300500 & -2.04819800 \\
\hline $\mathrm{C}$ & -2.72517000 & 3.97576700 & -1.29306000 & $\mathrm{O}$ & -1.66032100 & 0.20827300 & -3.20994500 \\
\hline $\mathrm{C}$ & -3.39886700 & 5.09435300 & -1.78890500 & $\mathrm{H}$ & 2.90336200 & 0.00325300 & 0.21666400 \\
\hline $\mathrm{C}$ & -2.79227600 & 6.35038300 & -1.75460600 & $\mathrm{H}$ & -2.92084900 & 1.06130000 & -0.23571500 \\
\hline $\mathrm{C}$ & -1.50624400 & 6.48654200 & -1.22512500 & $\mathrm{H}$ & 0.84889800 & -0.59394000 & 1.18505200 \\
\hline $\mathrm{C}$ & -0.83146100 & 5.37075300 & -0.73088000 & $\mathrm{C}$ & 5.07557800 & -3.79486700 & -2.31314000 \\
\hline $\mathrm{C}$ & -1.43710300 & 4.10359600 & -0.75409900 & $\mathrm{H}$ & 4.02672200 & -4.10578600 & -2.42188600 \\
\hline $\mathrm{C}$ & 2.13313100 & 3.47739700 & 0.22202000 & $\mathrm{H}$ & 5.69248900 & -4.67915000 & -2.51878900 \\
\hline $\mathrm{C}$ & 3.43794000 & 3.64983300 & -0.24907000 & $\mathrm{C}$ & 5.30776700 & -3.31641800 & -0.86500700 \\
\hline $\mathrm{C}$ & 3.78811600 & 3.20516900 & -1.52463300 & $\mathrm{H}$ & 5.04067100 & -4.10536900 & -0.15236700 \\
\hline $\mathrm{C}$ & 2.83028600 & 2.57706700 & -2.32610200 & $\mathrm{H}$ & 6.38493100 & -3.12113700 & -0.73481400 \\
\hline $\mathrm{C}$ & 1.53058800 & 2.39664500 & -1.85335400 & $\mathrm{C}$ & 5.38181400 & -2.67966600 & -3.32362300 \\
\hline
\end{tabular}




\begin{tabular}{|c|c|c|c|c|c|c|c|}
\hline $\mathrm{H}$ & 5.16674800 & -3.02085900 & -4.34444100 & C & -2.05247400 & -0.95159500 & -0.74929900 \\
\hline $\mathrm{H}$ & 6.45724100 & -2.44782400 & -3.29097600 & $\mathrm{H}$ & -2.05310600 & -1.98148500 & -0.35712100 \\
\hline $\mathrm{C}$ & 4.58038500 & -1.40820000 & -3.00861000 & $\mathrm{H}$ & -1.40385700 & -0.95559300 & -1.63048600 \\
\hline $\mathrm{H}$ & 3.50939700 & -1.60749500 & -3.15223100 & $\mathrm{C}$ & 3.46491700 & -1.71263300 & -0.05436700 \\
\hline $\mathrm{H}$ & 4.85001800 & -0.60475200 & -3.70652600 & $\mathrm{C}$ & 2.26342800 & -1.18142900 & -0.52402300 \\
\hline $\mathrm{C}$ & 4.53325600 & -2.04731200 & -0.56804700 & $\mathrm{C}$ & 1.95457400 & 0.17047600 & -0.32841900 \\
\hline $\mathrm{C}$ & 4.80865700 & -0.93394300 & -1.55643100 & $\mathrm{C}$ & 2.87514800 & 0.98410700 & 0.34477600 \\
\hline $\mathrm{H}$ & 5.86182700 & -0.62447600 & -1.45456800 & $\mathrm{C}$ & 4.07858600 & 0.45475600 & 0.81449600 \\
\hline $\mathrm{H}$ & 4.20217400 & -0.04657700 & -1.35562100 & $\mathrm{C}$ & 4.37712200 & -0.89496100 & 0.61745100 \\
\hline $\mathrm{C}$ & 4.59226500 & 1.15601500 & 3.67274500 & $\mathrm{H}$ & 3.69215500 & -2.76301200 & -0.21782600 \\
\hline $\mathrm{C}$ & 4.14284600 & 0.73863900 & 2.41831500 & $\mathrm{H}$ & 1.55769000 & -1.82186400 & -1.04928100 \\
\hline $\mathrm{C}$ & 3.27334600 & -0.35237100 & 2.29622400 & $\mathrm{H}$ & 2.64202700 & 2.03508600 & 0.48954100 \\
\hline $\mathrm{C}$ & 2.85713200 & -1.01585000 & 3.45858100 & $\mathrm{H}$ & 4.78431200 & 1.09802100 & 1.33413400 \\
\hline $\mathrm{C}$ & 3.30501800 & -0.60049200 & 4.71343400 & $\mathrm{H}$ & 5.31505200 & -1.30656100 & 0.98072400 \\
\hline $\mathrm{C}$ & 4.17576100 & 0.48628300 & 4.82522800 & $\mathrm{C}$ & 0.62575500 & 0.74177300 & -0.81814400 \\
\hline $\mathrm{H}$ & 5.26518100 & 2.00661000 & 3.75056300 & $\mathrm{O}$ & 0.75214800 & 2.11575800 & -1.20269400 \\
\hline $\mathrm{H}$ & 4.46531200 & 1.26654900 & 1.52362100 & $\mathrm{C}$ & -0.41033500 & 0.70109600 & 0.27622600 \\
\hline $\mathrm{H}$ & 2.17249800 & -1.85393200 & 3.36815200 & $\mathrm{H}$ & -0.16301000 & 1.34366900 & 1.12170500 \\
\hline $\mathrm{H}$ & 2.97327800 & -1.12538300 & 5.60600500 & & & & \\
\hline $\mathrm{H}$ & 4.52327300 & 0.81101600 & 5.80256500 & \multirow{2}{*}{\multicolumn{4}{|c|}{$\begin{array}{l}\text { TS24 } \\
\text { imaginary frequency }=-613.11 \mathrm{~cm}^{-1}\end{array}$}} \\
\hline $\mathrm{C}$ & 2.81997400 & -0.83596700 & 0.91890500 & & & & \\
\hline $\mathrm{O}$ & 1.47715000 & -1.32115000 & 0.94326400 & $\mathrm{Ru}$ & 0.88937400 & 0.59285600 & -0.09935500 \\
\hline $\mathrm{C}$ & 3.68057600 & -1.99020400 & 0.46508800 & $\mathrm{P}$ & 2.75005500 & -0.74530700 & -0.65702200 \\
\hline \multirow[t]{2}{*}{$\mathrm{H}$} & 3.57037300 & -2.87476600 & 1.09402900 & $\mathrm{P}$ & -0.85173700 & 2.14026000 & -0.39681000 \\
\hline & & & & $\mathrm{N}$ & 0.13649500 & -0.17764400 & -2.08916300 \\
\hline bpro1 & & & & $\mathrm{C}$ & 2.23875500 & -1.42551600 & -2.32414200 \\
\hline $\mathrm{H}$ & 0.29384500 & 0.14326200 & -1.67817000 & $\mathrm{C}$ & 1.27751400 & -0.44227800 & -2.99802800 \\
\hline $\mathrm{H}$ & 1.50923000 & 2.17349700 & -1.80872600 & $\mathrm{C}$ & -0.98429300 & 2.07485900 & -2.25824600 \\
\hline $\mathrm{C}$ & -3.89692000 & 0.43347500 & 1.12303900 & $\mathrm{C}$ & -0.96496200 & 0.61431100 & -2.71372500 \\
\hline $\mathrm{H}$ & -3.90367400 & 1.45893300 & 0.72814800 & $\mathrm{C}$ & 5.46228900 & -0.69429600 & -1.49209300 \\
\hline $\mathrm{H}$ & -4.54901400 & 0.42783600 & 2.00577500 & $\mathrm{C}$ & 6.68939700 & -0.09435500 & -1.76976300 \\
\hline $\mathrm{C}$ & -2.45641400 & 0.06440900 & 1.53329400 & $\mathrm{C}$ & 6.86348300 & 1.27573200 & -1.55459100 \\
\hline $\mathrm{H}$ & -2.06738300 & 0.78538200 & 2.26139400 & $\mathrm{C}$ & 5.80417500 & 2.03748500 & -1.06256200 \\
\hline $\mathrm{H}$ & -2.48076400 & -0.91564500 & 2.03739000 & $\mathrm{C}$ & 4.57059100 & 1.43764800 & -0.79373100 \\
\hline $\mathrm{C}$ & -4.43388500 & -0.52791100 & 0.05272700 & $\mathrm{C}$ & 4.38348600 & 0.06671400 & -1.00897100 \\
\hline $\mathrm{H}$ & -5.44094000 & -0.22477600 & -0.26112000 & $\mathrm{C}$ & 3.57647800 & -2.01659000 & 1.68159000 \\
\hline $\mathrm{H}$ & -4.53084200 & -1.53508000 & 0.48561800 & $\mathrm{C}$ & 4.01938600 & -3.07140300 & 2.47846800 \\
\hline $\mathrm{C}$ & -3.49584900 & -0.58724700 & -1.16145300 & $\mathrm{C}$ & 4.20007700 & -4.34148600 & 1.92449900 \\
\hline $\mathrm{H}$ & -3.48543200 & 0.39108100 & -1.66153300 & $\mathrm{C}$ & 3.94026500 & -4.54716100 & 0.56962900 \\
\hline $\mathrm{H}$ & -3.86448400 & -1.31542400 & -1.89521300 & $\mathrm{C}$ & 3.50347000 & -3.48881900 & -0.23174800 \\
\hline $\mathrm{C}$ & -1.53848600 & -0.02154800 & 0.32979500 & $\mathrm{C}$ & 3.31552600 & -2.21105300 & 0.31453000 \\
\hline
\end{tabular}




\begin{tabular}{|c|c|c|c|c|c|c|c|}
\hline $\mathrm{C}$ & 0.69999600 & 4.44488600 & 0.09765500 & $\mathrm{O}$ & 1.93280200 & 1.58117900 & 2.55320500 \\
\hline $\mathrm{C}$ & 0.91148900 & 5.80872600 & 0.30832600 & $\mathrm{H}$ & -0.15673800 & -0.69949000 & 0.63679700 \\
\hline $\mathrm{C}$ & -0.17170300 & 6.68665800 & 0.36833500 & $\mathrm{H}$ & 1.70750000 & 1.75246100 & -0.95256500 \\
\hline $\mathrm{C}$ & -1.46996100 & 6.19496600 & 0.21670700 & $\mathrm{H}$ & -0.27592800 & -1.09603100 & -1.86446800 \\
\hline $\mathrm{C}$ & -1.68393900 & 4.83246300 & 0.00625000 & $\mathrm{C}$ & -4.98577600 & -2.71250800 & -3.83677800 \\
\hline $\mathrm{C}$ & -0.59999500 & 3.94154300 & -0.06045900 & $\mathrm{C}$ & -3.76883400 & -2.59652600 & -3.16832600 \\
\hline $\mathrm{C}$ & -3.70748000 & 1.83653300 & -0.55091200 & $\mathrm{C}$ & -3.72156000 & -2.46257700 & -1.77133300 \\
\hline $\mathrm{C}$ & -4.96510800 & 1.61567800 & 0.01656200 & $\mathrm{C}$ & -4.93158400 & -2.47095900 & -1.05859000 \\
\hline $\mathrm{C}$ & -5.08709100 & 1.41256200 & 1.39162400 & $\mathrm{C}$ & -6.15112500 & -2.59011600 & -1.72529100 \\
\hline $\mathrm{C}$ & -3.94530900 & 1.41799800 & 2.19709400 & $\mathrm{C}$ & -6.18359900 & -2.70667400 & -3.11694000 \\
\hline $\mathrm{C}$ & -2.68877200 & 1.62290600 & 1.62857000 & $\mathrm{H}$ & -5.00219300 & -2.81192900 & -4.91942700 \\
\hline $\mathrm{C}$ & -2.55444600 & 1.84675500 & 0.24851000 & $\mathrm{H}$ & -2.82747100 & -2.61557000 & -3.70742000 \\
\hline $\mathrm{H}$ & 3.10468100 & -1.62299500 & -2.96444100 & $\mathrm{H}$ & -4.92213600 & -2.40549400 & 0.02435200 \\
\hline $\mathrm{H}$ & 1.71163600 & -2.37165900 & -2.15702600 & $\mathrm{H}$ & -7.07749600 & -2.60094700 & -1.15635100 \\
\hline $\mathrm{H}$ & 1.77679000 & 0.50601700 & -3.22118100 & $\mathrm{H}$ & -7.13453800 & -2.80087900 & -3.63524500 \\
\hline $\mathrm{H}$ & 0.91573600 & -0.87496700 & -3.94293500 & $\mathrm{C}$ & -2.36511100 & -2.34823800 & -1.11444700 \\
\hline $\mathrm{H}$ & -1.86753000 & 2.59467200 & -2.64682000 & $\mathrm{O}$ & -1.35817600 & -2.60946000 & -1.83097100 \\
\hline $\mathrm{H}$ & -0.10161000 & 2.60989800 & -2.62281800 & $\mathrm{C}$ & -2.30082500 & -1.90587400 & 0.24101900 \\
\hline $\mathrm{H}$ & -1.90451300 & 0.12894500 & -2.43513600 & $\mathrm{H}$ & -3.16830200 & -1.37632800 & 0.61815400 \\
\hline $\mathrm{H}$ & -0.88878000 & 0.55857100 & -3.80804100 & $\mathrm{C}$ & -1.17101200 & -1.95827200 & 1.07769800 \\
\hline $\mathrm{H}$ & 5.35091100 & -1.76541300 & -1.63861700 & $\mathrm{C}$ & -0.12804200 & -3.05231100 & 0.87925100 \\
\hline $\mathrm{H}$ & 7.51177600 & -0.69691000 & -2.14661000 & $\mathrm{H}$ & -0.54155600 & -3.98529700 & 1.28916300 \\
\hline $\mathrm{H}$ & 7.82178800 & 1.74299600 & -1.76580400 & $\mathrm{H}$ & 0.08380500 & -3.20721900 & -0.17642100 \\
\hline $\mathrm{H}$ & 5.93325100 & 3.10224400 & -0.88640500 & $\mathrm{H}$ & 0.79331700 & -2.83269700 & 1.41897500 \\
\hline $\mathrm{H}$ & 3.74070300 & 2.03043800 & -0.42491300 & $\mathrm{C}$ & -1.38804300 & -1.62309100 & 2.53539800 \\
\hline $\mathrm{H}$ & 3.42387300 & -1.03658700 & 2.12647900 & $\mathrm{C}$ & -0.34573800 & -1.13595800 & 3.34113700 \\
\hline $\mathrm{H}$ & 4.21511600 & -2.90333900 & 3.53405200 & $\mathrm{C}$ & -2.61968800 & -1.89165600 & 3.15583900 \\
\hline $\mathrm{H}$ & 4.53724800 & -5.16578000 & 2.54701400 & $\mathrm{C}$ & -0.53443300 & -0.89049300 & 4.69979800 \\
\hline $\mathrm{H}$ & 4.07452400 & -5.53261600 & 0.13137000 & $\mathrm{H}$ & 0.61442700 & -0.92525600 & 2.88545700 \\
\hline $\mathrm{H}$ & 3.30750000 & -3.67396900 & -1.28357300 & $\mathrm{C}$ & -2.81099600 & -1.65451200 & 4.51932600 \\
\hline $\mathrm{H}$ & 1.54079200 & 3.76078500 & 0.05296700 & $\mathrm{H}$ & -3.42886700 & -2.31450800 & 2.57001100 \\
\hline $\mathrm{H}$ & 1.92444200 & 6.18239200 & 0.43328200 & $\mathrm{C}$ & -1.77189200 & -1.14515400 & 5.29762900 \\
\hline $\mathrm{H}$ & -0.00665200 & 7.74748000 & 0.53781800 & $\mathrm{H}$ & 0.28728800 & -0.49480600 & 5.29117800 \\
\hline $\mathrm{H}$ & -2.31929200 & 6.87136200 & 0.26682900 & $\mathrm{H}$ & -3.77419800 & -1.87810400 & 4.97169600 \\
\hline $\mathrm{H}$ & -2.69921600 & 4.46219500 & -0.09773800 & $\mathrm{H}$ & -1.91966600 & -0.95600900 & 6.35779100 \\
\hline $\mathrm{H}$ & -3.64004400 & 1.99848200 & -1.62219900 & & & & \\
\hline $\mathrm{H}$ & -5.84623500 & 1.60107200 & -0.61925200 & \multicolumn{4}{|c|}{ TS25 } \\
\hline $\mathrm{H}$ & -6.06586300 & 1.24361900 & 1.83288000 & \multicolumn{4}{|c|}{ imaginary frequency $=-240.80 \mathrm{~cm}^{-1}$} \\
\hline $\mathrm{H}$ & -4.02654800 & 1.24104700 & 3.26545000 & $\mathrm{R}$ & -0.81391700 & 0.76596600 & 0.03519300 \\
\hline $\mathrm{H}$ & -1.80444700 & 1.59077600 & 2.25848900 & $\mathrm{P}$ & -2.81553000 & -0.45238100 & 0.38740600 \\
\hline $\mathrm{C}$ & 1.52211100 & 1.20598100 & 1.52699000 & $\mathrm{P}$ & 0.93223100 & 2.30787900 & 0.39093300 \\
\hline
\end{tabular}

\section{TS25}

imaginary frequency $=-240.80 \mathrm{~cm}^{-1}$

$\begin{array}{lrrc}\mathrm{Ru} & -0.81391700 & 0.76596600 & 0.03519300 \\ \mathrm{P} & -2.81553000 & -0.45238100 & 0.38740600 \\ \mathrm{P} & 0.93223100 & 2.30787900 & 0.39093300\end{array}$




\begin{tabular}{|c|c|c|c|c|c|c|c|}
\hline $\mathrm{N}$ & -0.66531400 & 0.54408000 & 2.18209200 & $\mathrm{H}$ & -3.59433400 & 2.07585400 & -0.90886000 \\
\hline $\mathrm{C}$ & -2.74074000 & -0.77284700 & 2.22403900 & $\mathrm{H}$ & -2.47670700 & -1.40375600 & -2.32980000 \\
\hline $\mathrm{C}$ & -1.93361800 & 0.34866200 & 2.89245600 & $\mathrm{H}$ & -2.88597900 & -3.55688300 & -3.46293400 \\
\hline $\mathrm{C}$ & 0.59957700 & 2.71694600 & 2.17785000 & $\mathrm{H}$ & -3.71783200 & -5.51382500 & -2.16962200 \\
\hline $\mathrm{C}$ & 0.29268500 & 1.39985000 & 2.90886300 & $\mathrm{H}$ & -4.14659000 & -5.28644600 & 0.26960600 \\
\hline $\mathrm{C}$ & -5.63573200 & -0.19933200 & 0.57583900 & $\mathrm{H}$ & -3.76127300 & -3.13650800 & 1.40307100 \\
\hline $\mathrm{C}$ & -6.86201800 & 0.43110900 & 0.37248000 & $\mathrm{H}$ & -1.15221900 & 4.32884400 & 0.09377000 \\
\hline $\mathrm{C}$ & -6.91617000 & 1.65789600 & -0.29528300 & $\mathrm{H}$ & -1.27216000 & 6.51326100 & -1.04411700 \\
\hline $\mathrm{C}$ & -5.73947700 & 2.24583500 & -0.75840500 & $\mathrm{H}$ & 0.68447600 & 7.36728000 & -2.32467100 \\
\hline $\mathrm{C}$ & -4.50915900 & 1.61645600 & -0.54947600 & $\mathrm{H}$ & 2.76870600 & 6.01292500 & -2.43274500 \\
\hline $\mathrm{C}$ & -4.44233700 & 0.38987200 & 0.12461500 & $\mathrm{H}$ & 2.90775600 & 3.84989100 & -1.26000700 \\
\hline $\mathrm{C}$ & -2.85567400 & -2.24575400 & -1.75803400 & $\mathrm{H}$ & 3.46110600 & 3.30067500 & 1.68415000 \\
\hline $\mathrm{C}$ & -3.08237100 & -3.46428200 & -2.39822400 & $\mathrm{H}$ & 5.80682500 & 2.56575800 & 1.50517700 \\
\hline $\mathrm{C}$ & -3.54845500 & -4.56263900 & -1.67229000 & $\mathrm{H}$ & 6.41502300 & 0.67044100 & 0.0149060 \\
\hline $\mathrm{C}$ & -3.78900800 & -4.43504700 & -0.30362900 & $\mathrm{H}$ & 4.63731500 & -0.50624300 & -1.28117200 \\
\hline $\mathrm{C}$ & -3.56948600 & -3.21356100 & 0.33695000 & $\mathrm{H}$ & 2.28599400 & 0.20286700 & -1.07307100 \\
\hline $\mathrm{C}$ & -3.10190300 & -2.10373700 & -0.38297200 & $\mathrm{C}$ & -0.84623500 & 0.82479800 & -1.83547500 \\
\hline $\mathrm{C}$ & -0.28225400 & 4.70305600 & -0.43833900 & $\mathrm{O}$ & -0.87457100 & 0.87029300 & -3.00085000 \\
\hline $\mathrm{C}$ & -0.35450400 & 5.93266500 & -1.09071000 & $\mathrm{H}$ & 0.48711400 & -2.31643600 & -1.33829000 \\
\hline $\mathrm{C}$ & 0.74332800 & 6.41184800 & -1.81021700 & $\mathrm{H}$ & -1.67884400 & 2.07926600 & 0.04021200 \\
\hline $\mathrm{C}$ & 1.91156800 & 5.65291800 & -1.86978200 & $\mathrm{H}$ & -0.04681500 & -0.71462900 & 1.73709000 \\
\hline $\mathrm{C}$ & 1.98985000 & 4.42546900 & -1.20669700 & $\mathrm{C}$ & 1.86837400 & -2.13401100 & 4.89428900 \\
\hline $\mathrm{C}$ & 0.89560900 & 3.93746900 & -0.47804700 & $\mathrm{C}$ & 1.27189200 & -2.15171900 & 3.63388800 \\
\hline $\mathrm{C}$ & 3.70798800 & 2.45882900 & 1.04313500 & $\mathrm{C}$ & 2.04860300 & -2.05677300 & 2.46565600 \\
\hline $\mathrm{C}$ & 5.03682500 & 2.05044700 & 0.93690000 & $\mathrm{C}$ & 3.44071600 & -1.92419600 & 2.60239300 \\
\hline $\mathrm{C}$ & 5.37762600 & 0.98402900 & 0.09881000 & $\mathrm{C}$ & 4.03705800 & -1.90732000 & 3.86323900 \\
\hline $\mathrm{C}$ & 4.38617300 & 0.32479000 & -0.62850800 & $\mathrm{C}$ & 3.25476200 & -2.01369400 & 5.01531400 \\
\hline $\mathrm{C}$ & 3.05313700 & 0.72849400 & -0.51426800 & $\mathrm{H}$ & 1.24956200 & -2.22071000 & 5.78407800 \\
\hline $\mathrm{C}$ & 2.69976900 & 1.80008600 & 0.31743800 & $\mathrm{H}$ & 0.19482500 & -2.26043300 & 3.54781000 \\
\hline $\mathrm{H}$ & -3.73763200 & -0.86629000 & 2.66752200 & $\mathrm{H}$ & 4.04927600 & -1.80827000 & 1.71098600 \\
\hline $\mathrm{H}$ & -2.21099100 & -1.72276000 & 2.35093000 & $\mathrm{H}$ & 5.11549700 & -1.79726000 & 3.94579400 \\
\hline $\mathrm{H}$ & -2.53798400 & 1.27167600 & 2.92288200 & $\mathrm{H}$ & 3.71965800 & -1.99655600 & 5.99758900 \\
\hline $\mathrm{H}$ & -1.74935900 & 0.06057700 & 3.94124800 & $\mathrm{C}$ & 1.40616300 & -2.11335600 & 1.12208400 \\
\hline $\mathrm{H}$ & 1.42558500 & 3.26433200 & 2.64823700 & $\mathrm{O}$ & 0.20833800 & -1.47898300 & 0.99235100 \\
\hline $\mathrm{H}$ & -0.28237500 & 3.36716100 & 2.18182000 & $\mathrm{C}$ & 1.94915400 & -2.82304000 & 0.11076700 \\
\hline $\mathrm{H}$ & 1.23527900 & 0.85195000 & 3.05349700 & $\mathrm{H}$ & 2.86674600 & -3.36619600 & 0.31984700 \\
\hline $\mathrm{H}$ & -0.08364400 & 1.63292100 & 3.91506600 & $\mathrm{C}$ & 1.34149600 & -2.99653200 & -1.26164400 \\
\hline $\mathrm{H}$ & -5.61198000 & -1.16364000 & 1.07664300 & $\mathrm{C}$ & 0.80210000 & -4.43557400 & -1.44674700 \\
\hline $\mathrm{H}$ & -7.77565400 & -0.03674000 & 0.72977900 & $\mathrm{H}$ & 1.60398700 & -5.17638800 & -1.34169500 \\
\hline $\mathrm{H}$ & -7.87258100 & 2.14825300 & -0.45652400 & $\mathrm{H}$ & 0.03878900 & -4.65204700 & -0.69212500 \\
\hline $\mathrm{H}$ & -5.77420600 & 3.19601500 & -1.28484200 & $\mathrm{H}$ & 0.35483600 & -4.56186100 & -2.43988800 \\
\hline
\end{tabular}




$\begin{array}{llll}\mathrm{C} & 2.32358500 & -2.63783300 & -2.37584600 \\ \mathrm{C} & 1.99101400 & -1.67155400 & -3.33597100 \\ \mathrm{C} & 3.57154900 & -3.27286400 & -2.48645000 \\ \mathrm{C} & 2.87480700 & -1.34305100 & -4.36795700 \\ \mathrm{H} & 1.02884600 & -1.16824600 & -3.27768200 \\ \mathrm{C} & 4.45706100 & -2.94927300 & -3.51548100 \\ \mathrm{H} & 3.85529900 & -4.03219200 & -1.76152000 \\ \mathrm{C} & 4.11261800 & -1.97979300 & -4.46174500 \\ \mathrm{H} & 2.59161100 & -0.58815900 & -5.09722200 \\ \mathrm{H} & 5.41654900 & -3.45702300 & -3.58061300 \\ \mathrm{H} & 4.80171400 & -1.72693600 & -5.26338800\end{array}$

\section{bpro2}

$\mathrm{H}$

C

C

C

C

C

C

$\mathrm{H}$

$\mathrm{H}$

$\mathrm{H}$

H

H

C

O

C

C

$\mathrm{H}$

H

H

C

C

C

C

H

C

$\mathrm{H}$

C

$\mathrm{H}$

$\begin{array}{lrrr}\mathrm{H} & -4.40771600 & -2.21015900 & 1.48335600 \\ \mathrm{H} & -5.83708200 & -1.80666100 & -0.51391900 \\ \mathrm{C} & -0.02902000 & 0.12837400 & 0.10624800 \\ \mathrm{H} & 0.06069900 & -0.47474500 & 1.02002500 \\ \mathrm{H} & -0.15128600 & -0.59630500 & -0.71192700\end{array}$

\section{TS26}

imaginary frequency $=-624.08 \mathrm{~cm}^{-1}$

\begin{tabular}{|c|c|c|c|}
\hline $\mathrm{Ru}$ & 0.51663000 & 0.61065700 & -0.09013000 \\
\hline $\mathrm{P}$ & 2.68958900 & -0.15251900 & -0.59875400 \\
\hline $\mathrm{P}$ & -1.35448600 & 2.01873500 & -0.30847000 \\
\hline $\mathrm{N}$ & 0.23269600 & 0.36192900 & -2.31258100 \\
\hline $\mathrm{C}$ & 2.56877700 & -0.42068800 & -2.44672000 \\
\hline $\mathrm{C}$ & 1.51810000 & 0.52772400 & -3.03047300 \\
\hline $\mathrm{C}$ & -1.21823300 & 2.41451700 & -2.12861500 \\
\hline $\mathrm{C}$ & -0.88981800 & 1.14176200 & -2.91107800 \\
\hline $\mathrm{C}$ & 3.96063000 & 2.27100000 & 0.20001900 \\
\hline $\mathrm{C}$ & 5.04267700 & 3.14688000 & 0.32492300 \\
\hline $\mathrm{C}$ & 6.29242000 & 2.79758400 & -0.18536200 \\
\hline $\mathrm{C}$ & 6.46079500 & 1.56096100 & -0.81425500 \\
\hline $\mathrm{C}$ & 5.38397800 & 0.68318400 & -0.92825600 \\
\hline $\mathrm{C}$ & 4.11566900 & 1.03080200 & -0.43139300 \\
\hline $\mathrm{C}$ & 3.42611900 & -1.90306300 & 1.44698100 \\
\hline $\mathrm{C}$ & 4.02067300 & -3.03007500 & 2.01382500 \\
\hline $\mathrm{C}$ & 4.66440800 & -3.96767900 & 1.20270200 \\
\hline $\mathrm{C}$ & 4.71214000 & -3.77058200 & -0.17777600 \\
\hline $\mathrm{C}$ & 4.12328700 & -2.63854100 & -0.74630300 \\
\hline $\mathrm{C}$ & 3.47449200 & -1.69067200 & 0.05935400 \\
\hline $\mathrm{C}$ & -0.25504700 & 4.12372900 & 1.24415500 \\
\hline $\mathrm{C}$ & -0.23841300 & 5.40465800 & 1.80198300 \\
\hline $\mathrm{C}$ & -1.30316100 & 6.27923400 & 1.58475600 \\
\hline $\mathrm{C}$ & -2.39182100 & 5.86690800 & 0.81178200 \\
\hline $\mathrm{C}$ & -2.41400800 & 4.58520900 & 0.26329900 \\
\hline $\mathrm{C}$ & -1.34170900 & 3.69973800 & 0.46774800 \\
\hline $\mathrm{C}$ & -4.06130500 & 1.37962100 & -1.05556200 \\
\hline $\mathrm{C}$ & -5.38517300 & 1.03690300 & -0.76305100 \\
\hline $\mathrm{C}$ & -5.78701500 & 0.84869200 & 0.55892800 \\
\hline $\mathrm{C}$ & -4.85753400 & 1.00362800 & 1.59144300 \\
\hline $\mathrm{C}$ & -3.53862700 & 1.34573100 & 1.29931800 \\
\hline $\mathrm{C}$ & -3.12159600 & 1.54459700 & -0.02856600 \\
\hline $\mathrm{H}$ & 3.53336300 & -0.27619800 & -2.94458200 \\
\hline
\end{tabular}


$\mathrm{H}$

H

H

H

H

C

O

H

H

H

C

C

H

H

C

H

$\mathrm{H}$

C

$\mathrm{H}$

\begin{tabular}{|c|c|c|c|c|c|c|c|}
\hline $\mathrm{H}$ & 2.25162400 & -1.45650400 & -2.61268600 & $\mathrm{H}$ & -5.57127700 & -3.11979600 & -0.51767400 \\
\hline $\mathrm{H}$ & 1.84046500 & 1.56955800 & -2.93032400 & $\mathrm{C}$ & -0.94528700 & -2.55999400 & -0.00798600 \\
\hline $\mathrm{H}$ & 1.38411000 & 0.31063700 & -4.10109900 & $\mathrm{C}$ & 0.33398500 & -3.18317800 & -0.56426600 \\
\hline $\mathrm{H}$ & -2.11606700 & 2.90135600 & -2.52686200 & $\mathrm{H}$ & 1.18112300 & -2.97272600 & 0.08691400 \\
\hline $\mathrm{H}$ & -0.39563800 & 3.13384600 & -2.19792000 & $\mathrm{H}$ & 0.20683100 & -4.27382700 & -0.61487100 \\
\hline $\mathrm{H}$ & -1.75321500 & 0.47103400 & -2.92969400 & $\mathrm{H}$ & 0.53829200 & -2.83690400 & -1.57313900 \\
\hline $\mathrm{H}$ & -0.65576700 & 1.39007600 & -3.95540600 & $\mathrm{C}$ & -1.11059400 & -2.80646200 & 1.47784100 \\
\hline $\mathrm{H}$ & 2.98430500 & 2.54993800 & 0.58011900 & $\mathrm{C}$ & -0.86556600 & -4.08679000 & 2.00250500 \\
\hline $\mathrm{H}$ & 4.90374200 & 4.10404800 & 0.82084900 & $\mathrm{C}$ & -1.54131000 & -1.80914400 & 2.36416000 \\
\hline $\mathrm{H}$ & 7.13273000 & 3.48048300 & -0.09143600 & $\mathrm{C}$ & -1.04425700 & -4.35736400 & 3.36095400 \\
\hline $\mathrm{H}$ & 7.43261500 & 1.27672700 & -1.20947900 & $\mathrm{H}$ & -0.53838200 & -4.88654400 & 1.34536600 \\
\hline $\mathrm{H}$ & 5.54062700 & -0.28574300 & -1.39439100 & $\mathrm{C}$ & -1.71443900 & -2.07384500 & 3.72239300 \\
\hline $\mathrm{H}$ & 2.90718500 & -1.19352800 & 2.08548400 & $\mathrm{H}$ & -1.72347800 & -0.81434800 & 1.97545500 \\
\hline $\mathrm{H}$ & 3.97025700 & -3.18038100 & 3.08872200 & $\mathrm{C}$ & -1.46696200 & -3.35111000 & 4.22948000 \\
\hline $\mathrm{H}$ & 5.12060200 & -4.84977400 & 1.64389400 & $\mathrm{H}$ & -0.84812200 & -5.35818900 & 3.73807700 \\
\hline $\mathrm{H}$ & 5.20550100 & -4.49843100 & -0.81673300 & $\mathrm{H}$ & -2.03610800 & -1.27617200 & 4.38747200 \\
\hline $\mathrm{H}$ & 4.16973800 & -2.50752100 & -1.82354900 & $\mathrm{H}$ & -1.59841200 & -3.55782500 & 5.28849500 \\
\hline $\mathrm{H}$ & 0.57599700 & 3.44728000 & 1.40686300 & $\mathrm{C}$ & -3.49145100 & -2.69504800 & -0.10579100 \\
\hline $\mathrm{H}$ & 0.60831000 & 5.71529600 & 2.40842900 & $\mathrm{H}$ & -3.63969400 & -1.85170300 & 0.57966600 \\
\hline $\mathrm{H}$ & -1.28904100 & 7.27581600 & 2.01826000 & $\mathrm{H}$ & -3.50738100 & -3.59183000 & 0.53122200 \\
\hline $\mathrm{H}$ & -3.22684600 & 6.54136100 & 0.64084800 & $\mathrm{C}$ & -2.06034500 & -2.52152800 & -2.22767500 \\
\hline $\mathrm{H}$ & -3.27573200 & 4.26929300 & -0.31820300 & $\mathrm{O}$ & -1.08552500 & -2.07129200 & -2.88625600 \\
\hline
\end{tabular}

\section{TS27}

$\begin{array}{lrrr}\text { imaginary frequency }=-442.24 \mathrm{~cm}^{-1} & \\ \mathrm{Ru} & -1.13356100 & 0.56693900 & 0.11501000 \\ \mathrm{P} & 0.32693200 & 2.40651300 & -0.17927200 \\ \mathrm{P} & -2.65262000 & -1.14830500 & -0.47252800 \\ \mathrm{~N} & -0.10371500 & -0.12105400 & -1.67948500 \\ \mathrm{C} & 1.25376000 & 1.92437300 & -1.72920800 \\ \mathrm{C} & 0.39176400 & 0.95407800 & -2.54703500 \\ \mathrm{C} & -2.26045000 & -1.25979000 & -2.29345500 \\ \mathrm{C} & -0.72790800 & -1.23193900 & -2.41949100 \\ \mathrm{C} & -1.75721900 & 4.30118400 & -0.47230900 \\ \mathrm{C} & -2.28919400 & 5.56173400 & -0.75779600 \\ \mathrm{C} & -1.44898200 & 6.61047000 & -1.13023200 \\ \mathrm{C} & -0.06973100 & 6.39833800 & -1.21149400 \\ \mathrm{C} & 0.46159900 & 5.14261700 & -0.92290100 \\ \mathrm{C} & -0.37674700 & 4.07575300 & -0.55614200 \\ \mathrm{C} & 1.29681700 & 2.78386400 & 2.41869500 \\ \mathrm{C} & 2.24599200 & 3.09086400 & 3.39429500\end{array}$




\begin{tabular}{|c|c|c|c|c|c|c|c|}
\hline $\mathrm{C}$ & 3.55615700 & 3.40406200 & 3.02579500 & $\mathrm{H}$ & -2.02957000 & -6.59614200 & 1.24428000 \\
\hline $\mathrm{C}$ & 3.91137000 & 3.41123700 & 1.67627800 & $\mathrm{H}$ & -4.22055800 & -5.84266500 & 0.34106000 \\
\hline $\mathrm{C}$ & 2.96060000 & 3.10941400 & 0.69852800 & $\mathrm{H}$ & -4.53094800 & -3.50222800 & -0.37708800 \\
\hline $\mathrm{C}$ & 1.64073200 & 2.79422700 & 1.05772900 & $\mathrm{C}$ & -1.88116000 & 1.07079000 & 1.75210200 \\
\hline $\mathrm{C}$ & -4.99947900 & -0.35748300 & 0.84128400 & $\mathrm{O}$ & -2.34958100 & 1.38444000 & 2.77382400 \\
\hline $\mathrm{C}$ & -6.36684000 & -0.11018700 & 0.96178100 & $\mathrm{H}$ & 2.13460800 & -1.72085000 & -1.48932700 \\
\hline $\mathrm{C}$ & -7.21600100 & -0.30910400 & -0.12940800 & $\mathrm{H}$ & -2.25315100 & 1.41282000 & -0.61163100 \\
\hline $\mathrm{C}$ & -6.69087700 & -0.75923900 & -1.34112300 & $\mathrm{H}$ & 0.81469500 & -0.57851000 & -0.62399000 \\
\hline $\mathrm{C}$ & -5.32237700 & -1.01394100 & -1.46137800 & $\mathrm{C}$ & 2.67423300 & -2.36446800 & 0.47532000 \\
\hline $\mathrm{C}$ & -4.46020400 & -0.81627900 & -0.37165000 & $\mathrm{C}$ & 1.63017000 & -1.44692100 & 2.58783900 \\
\hline $\mathrm{C}$ & -1.28103500 & -3.33148600 & 0.62807000 & $\mathrm{H}$ & 0.55462300 & -1.40083000 & 2.80450300 \\
\hline $\mathrm{C}$ & -1.10660900 & -4.65817200 & 1.02857200 & $\mathrm{H}$ & 2.04012400 & -0.48459500 & 2.92955300 \\
\hline $\mathrm{C}$ & -2.16196500 & -5.56550000 & 0.92566200 & $\mathrm{C}$ & 2.28730800 & -2.60758100 & 3.34370700 \\
\hline $\mathrm{C}$ & -3.39264000 & -5.14245700 & 0.41747000 & $\mathrm{H}$ & 1.65992700 & -3.50662300 & 3.25629300 \\
\hline $\mathrm{C}$ & -3.56708300 & -3.81944100 & 0.00963300 & $\mathrm{H}$ & 2.34877200 & -2.37158000 & 4.41341800 \\
\hline $\mathrm{C}$ & -2.51024900 & -2.89979000 & 0.10705300 & $\mathrm{C}$ & 3.67132800 & -2.90511300 & 2.76192400 \\
\hline $\mathrm{H}$ & 1.53676000 & 2.80180800 & -2.32002600 & $\mathrm{H}$ & 4.30051700 & -2.00787900 & 2.84633300 \\
\hline $\mathrm{H}$ & 2.16790000 & 1.41059800 & -1.41454900 & $\mathrm{H}$ & 4.17128500 & -3.70063000 & 3.32918900 \\
\hline $\mathrm{H}$ & -0.43129200 & 1.50611800 & -3.03091600 & $\mathrm{C}$ & 2.86383400 & -2.40407700 & -1.03754300 \\
\hline $\mathrm{H}$ & 1.01467800 & 0.54119500 & -3.35831800 & $\mathrm{C}$ & 2.55365100 & -3.80130200 & -1.60921400 \\
\hline $\mathrm{H}$ & -2.68820000 & -2.15744200 & -2.75809700 & $\mathrm{H}$ & 2.58223900 & -3.80345100 & -2.70499600 \\
\hline $\mathrm{H}$ & -2.69391100 & -0.37232300 & -2.76863600 & $\mathrm{H}$ & 3.26202500 & -4.55824900 & -1.25409300 \\
\hline $\mathrm{H}$ & -0.33568800 & -2.18663300 & -2.03861200 & $\mathrm{H}$ & 1.55047200 & -4.11250800 & -1.29862200 \\
\hline $\mathrm{H}$ & -0.45459400 & -1.19216100 & -3.48406900 & $\mathrm{C}$ & 4.24487500 & -1.86126000 & -1.43787200 \\
\hline $\mathrm{H}$ & -2.41248000 & 3.48542500 & -0.18583300 & $\mathrm{C}$ & 5.11684300 & -2.53615200 & -2.30150000 \\
\hline $\mathrm{H}$ & -3.36214100 & 5.71971100 & -0.68762500 & $\mathrm{C}$ & 4.65386500 & -0.61201500 & -0.93889900 \\
\hline $\mathrm{H}$ & -1.86317400 & 7.59031500 & -1.35289800 & $\mathrm{C}$ & 6.35092200 & -1.98321500 & -2.66059100 \\
\hline $\mathrm{H}$ & 0.59216700 & 7.21247600 & -1.49501100 & $\mathrm{H}$ & 4.84114100 & -3.50499300 & -2.70546800 \\
\hline $\mathrm{H}$ & 1.53763700 & 4.99861500 & -0.97096200 & $\mathrm{C}$ & 5.88016300 & -0.05555700 & -1.29752900 \\
\hline $\mathrm{H}$ & 0.28551200 & 2.52567200 & 2.71807500 & $\mathrm{H}$ & 4.00325500 & -0.08303400 & -0.24689500 \\
\hline $\mathrm{H}$ & 1.96208400 & 3.07766000 & 4.44317100 & $\mathrm{C}$ & 6.73804000 & -0.74039700 & -2.16316000 \\
\hline $\mathrm{H}$ & 4.29680200 & 3.63682300 & 3.78616600 & $\mathrm{H}$ & 7.00892300 & -2.53150300 & -3.33051200 \\
\hline $\mathrm{H}$ & 4.92976300 & 3.65034500 & 1.38106800 & $\mathrm{H}$ & 6.17063000 & 0.91220500 & -0.89527300 \\
\hline $\mathrm{H}$ & 3.25930000 & 3.11844000 & -0.34567100 & $\mathrm{H}$ & 7.69673100 & -0.31058000 & -2.44175200 \\
\hline $\mathrm{H}$ & -4.34819300 & -0.17987000 & 1.69136700 & $\mathrm{C}$ & 3.55164000 & -3.30506000 & 1.28664200 \\
\hline $\mathrm{H}$ & -6.76691100 & 0.24592800 & 1.90724400 & $\mathrm{H}$ & 4.55197200 & -3.34045200 & 0.83307000 \\
\hline $\mathrm{H}$ & -8.28030400 & -0.11019000 & -0.03637700 & $\mathrm{H}$ & 3.16565100 & -4.33637100 & 1.22582000 \\
\hline $\mathrm{H}$ & -7.34396600 & -0.91336900 & -2.19611600 & $\mathrm{C}$ & 1.80962400 & -1.52375700 & 1.08667200 \\
\hline $\mathrm{H}$ & -4.93265100 & -1.36717600 & -2.41125100 & $\mathrm{O}$ & 1.03103600 & -0.59806600 & 0.45605000 \\
\hline $\mathrm{H}$ & -0.46087600 & -2.62812100 & 0.72051000 & & & & \\
\hline $\mathrm{H}$ & -0.14645000 & -4.97517900 & 1.42671600 & bpro3 & & & \\
\hline
\end{tabular}




\begin{tabular}{|c|c|c|c|c|c|c|c|}
\hline C & 3.18082700 & 0.15485300 & 0.63379900 & $\mathrm{C}$ & 1.11309200 & 0.59689500 & -2.78119600 \\
\hline $\mathrm{H}$ & 4.20224000 & -0.22796900 & 0.71738700 & $\mathrm{C}$ & -2.33096600 & 0.91688800 & -2.25833600 \\
\hline $\mathrm{H}$ & 2.74204500 & 0.15534200 & 1.64334500 & $\mathrm{C}$ & -1.29474400 & -0.08566100 & -2.77064600 \\
\hline $\mathrm{C}$ & 3.14210200 & 1.59131800 & 0.07058900 & $\mathrm{C}$ & 2.07711200 & 3.95134800 & -0.11068500 \\
\hline $\mathrm{H}$ & 3.70157900 & 1.61917500 & -0.87486000 & $\mathrm{C}$ & 2.59492200 & 5.24678200 & -0.19651800 \\
\hline $\mathrm{H}$ & 3.65678500 & 2.27246800 & 0.75911400 & $\mathrm{C}$ & 3.92932100 & 5.44869900 & -0.54723400 \\
\hline C & 1.70007500 & 2.05386500 & -0.18001500 & $\mathrm{C}$ & 4.75073900 & 4.34772300 & -0.80523000 \\
\hline $\mathrm{H}$ & 1.17134800 & 2.14820700 & 0.77867800 & $\mathrm{C}$ & 4.23676600 & 3.05545100 & -0.71068400 \\
\hline $\mathrm{H}$ & 1.69973900 & 3.05446800 & -0.62987400 & C & 2.88964400 & 2.84072100 & -0.37059900 \\
\hline C & 0.07046000 & -2.04248800 & 1.18003100 & $\mathrm{C}$ & 3.42141900 & 0.32419400 & 2.11902600 \\
\hline $\mathrm{H}$ & -0.62806700 & -2.17997100 & 2.01207700 & $\mathrm{C}$ & 4.40352100 & -0.25046100 & 2.92576300 \\
\hline $\mathrm{H}$ & -0.18425400 & -2.76983400 & 0.40123800 & $\mathrm{C}$ & 5.50447300 & -0.88692100 & 2.34769000 \\
\hline $\mathrm{H}$ & 1.07749000 & -2.28561500 & 1.53465700 & $\mathrm{C}$ & 5.61971900 & -0.94134100 & 0.95827100 \\
\hline C & -1.44731200 & -0.22581200 & 0.29896900 & $\mathrm{C}$ & 4.64171900 & -0.35681400 & 0.14933300 \\
\hline C & -2.15773100 & -0.90490500 & -0.70348300 & $\mathrm{C}$ & 3.53028300 & 0.28379400 & 0.71933100 \\
\hline C & -2.10505600 & 0.79677900 & 0.99543800 & $\mathrm{C}$ & -2.70637500 & 3.54736200 & 0.59897200 \\
\hline C & -3.47938600 & -0.57074600 & -0.99866500 & $\mathrm{C}$ & -3.41155400 & 4.72821500 & 0.84571700 \\
\hline $\mathrm{H}$ & -1.67589900 & -1.70541900 & -1.25946400 & $\mathrm{C}$ & -4.74897100 & 4.84020300 & 0.46547000 \\
\hline C & -3.42909500 & 1.13452000 & 0.70484000 & $\mathrm{C}$ & -5.38492500 & 3.76291800 & -0.15736700 \\
\hline $\mathrm{H}$ & -1.57471000 & 1.33349300 & 1.77941100 & $\mathrm{C}$ & -4.68530700 & 2.58023600 & -0.39379600 \\
\hline C & -4.12153400 & 0.45126100 & -0.29491200 & $\mathrm{C}$ & -3.33431500 & 2.46095000 & -0.02428100 \\
\hline $\mathrm{H}$ & -4.00960200 & -1.11080800 & -1.77890100 & $\mathrm{C}$ & -4.15762500 & -1.32585700 & -0.81165100 \\
\hline $\mathrm{H}$ & -3.91690400 & 1.93105200 & 1.26085000 & $\mathrm{C}$ & -5.09076900 & -2.27412400 & -0.38309600 \\
\hline $\mathrm{H}$ & -5.15144100 & 0.71093400 & -0.52450500 & $\mathrm{C}$ & -5.54322500 & -2.27025600 & 0.93571900 \\
\hline C & 0.95561600 & 1.07604000 & -1.10272300 & $\mathrm{C}$ & -5.05557600 & -1.31355900 & 1.83096400 \\
\hline $\mathrm{H}$ & -0.07481700 & 1.40964600 & -1.26711000 & $\mathrm{C}$ & -4.12420100 & -0.36889200 & 1.40512600 \\
\hline $\mathrm{H}$ & 1.44608400 & 1.08108300 & -2.08631400 & $\mathrm{C}$ & -3.66767300 & -0.35832000 & 0.07548600 \\
\hline C & 2.37923000 & -0.80869400 & -0.23409900 & $\mathrm{H}$ & 3.23641000 & 0.97160100 & -2.53748100 \\
\hline $\mathrm{O}$ & 2.85416800 & -1.85118000 & -0.64764900 & $\mathrm{H}$ & 2.69153700 & -0.60215700 & -1.93265800 \\
\hline $\mathrm{C}$ & -0.01043400 & -0.59850700 & 0.64397600 & $\mathrm{H}$ & 0.86368100 & 1.65009600 & -2.94048800 \\
\hline $\mathrm{H}$ & 0.30785600 & 0.07164000 & 1.45294300 & $\mathrm{H}$ & 1.21253000 & 0.11602600 & -3.76644600 \\
\hline C & 0.94831400 & -0.38153200 & -0.57412700 & $\mathrm{H}$ & -3.30920300 & 0.72352200 & -2.71410700 \\
\hline \multirow[t]{2}{*}{$\mathrm{H}$} & 0.62072700 & -1.05415400 & -1.37519000 & $\mathrm{H}$ & -2.04142900 & 1.94128600 & -2.51570600 \\
\hline & & & & $\mathrm{H}$ & -1.66112500 & -1.10896700 & -2.66099900 \\
\hline \multicolumn{4}{|c|}{ TS28 } & $\mathrm{H}$ & -1.10134100 & 0.09137100 & -3.83713200 \\
\hline \multicolumn{4}{|c|}{ imaginary frequency $=-454.98 \mathrm{~cm}^{-1}$} & $\mathrm{H}$ & 1.03535100 & 3.79086200 & 0.14556400 \\
\hline $\mathrm{R}$ & -0.09618400 & 0.78869000 & 0.07258000 & $\mathrm{H}$ & 1.95041500 & 6.09710400 & 0.01075800 \\
\hline $\mathrm{P}$ & 2.20507000 & 1.11534900 & -0.26516500 & $\mathrm{H}$ & 4.33100200 & 6.45629200 & -0.61593400 \\
\hline $\mathrm{P}$ & -2.40187900 & 0.90392900 & -0.39049000 & $\mathrm{H}$ & 5.79383400 & 4.49520400 & -1.07290400 \\
\hline $\mathrm{N}$ & 0.00110000 & -0.02215400 & -2.01642200 & $\mathrm{H}$ & 4.89663400 & 2.21073500 & -0.88844900 \\
\hline C & 2.42684000 & 0.45890900 & -2.00757700 & $\mathrm{H}$ & 2.55861600 & 0.79626300 & 2.58006500 \\
\hline
\end{tabular}




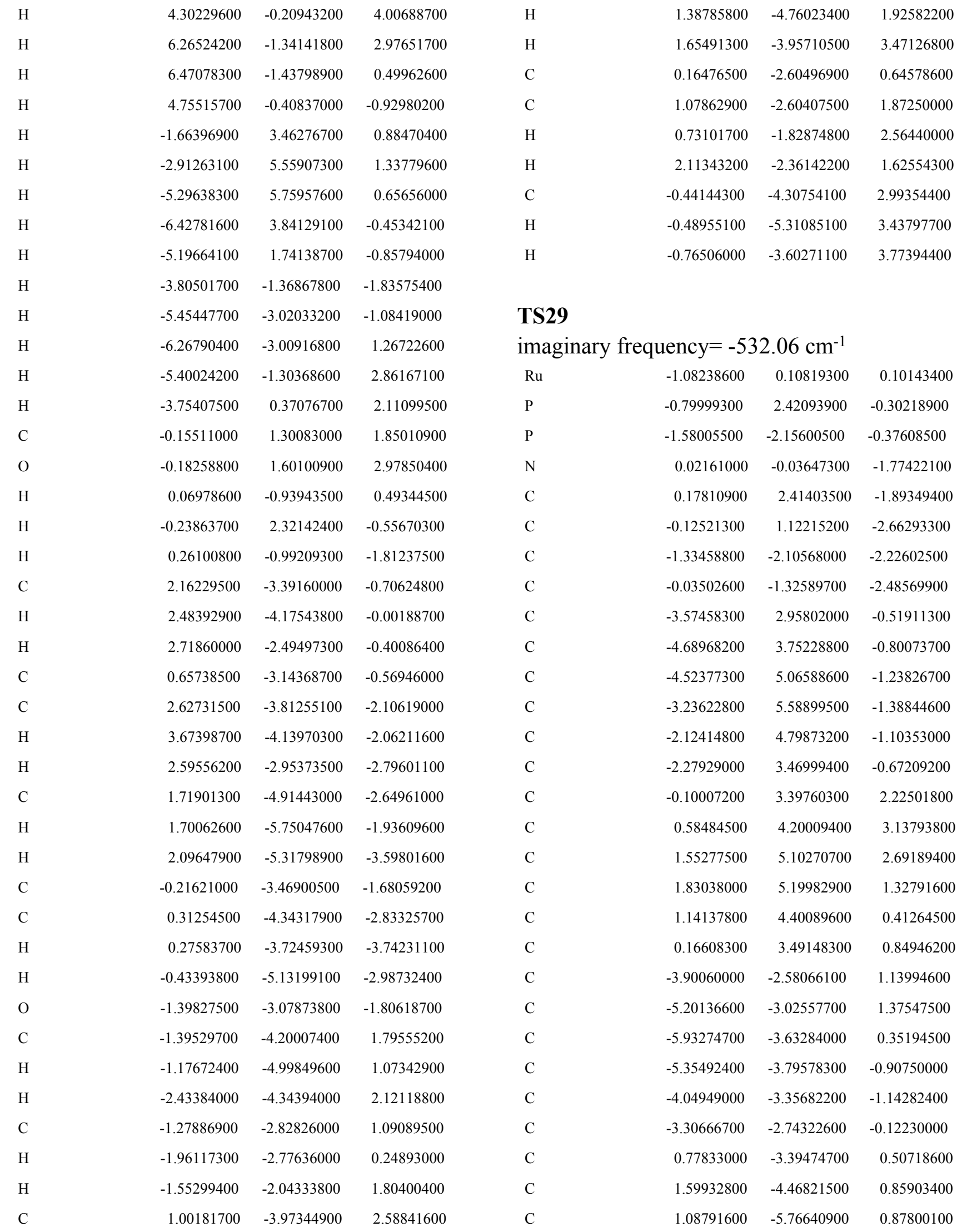




\begin{tabular}{|c|c|c|c|c|c|c|c|}
\hline $\mathrm{C}$ & -0.24881800 & -5.98846300 & 0.53888400 & $\mathrm{H}$ & 5.25393000 & -1.77995200 & 3.18696200 \\
\hline $\mathrm{C}$ & -1.06895600 & -4.91891200 & 0.17757200 & $\mathrm{H}$ & 3.73171300 & -2.47437200 & 2.62883900 \\
\hline $\mathrm{C}$ & -0.56265100 & -3.60896700 & 0.15388600 & $\mathrm{C}$ & 3.56206500 & -0.40547400 & 3.20608600 \\
\hline $\mathrm{H}$ & -0.03041700 & 3.29970100 & -2.50289700 & $\mathrm{H}$ & 4.17471600 & 0.50638400 & 3.16857400 \\
\hline $\mathrm{H}$ & 1.23670400 & 2.42375900 & -1.61554800 & $\mathrm{H}$ & 3.33174000 & -0.58978800 & 4.26276700 \\
\hline $\mathrm{H}$ & -1.13649000 & 1.18032900 & -3.09986700 & $\mathrm{C}$ & 2.47190500 & -0.25307000 & 0.91402800 \\
\hline $\mathrm{H}$ & 0.57932200 & 1.05295900 & -3.50926300 & $\mathrm{C}$ & 2.27014500 & -0.19099100 & 2.41245600 \\
\hline $\mathrm{H}$ & -1.29910400 & -3.10922600 & -2.66901400 & $\mathrm{H}$ & 1.51691800 & -0.94221600 & 2.69753600 \\
\hline $\mathrm{H}$ & -2.18892900 & -1.56173100 & -2.64486800 & $\mathrm{H}$ & 1.82191600 & 0.78223500 & 2.64901500 \\
\hline $\mathrm{H}$ & 0.81110400 & -1.95495400 & -2.17028000 & $\mathrm{O}$ & 1.37809400 & 0.23969200 & 0.26182000 \\
\hline $\mathrm{H}$ & 0.08345400 & -1.17752700 & -3.56907000 & $\mathrm{C}$ & 5.55272700 & 1.17480600 & -1.57623000 \\
\hline $\mathrm{H}$ & -3.70512400 & 1.93523800 & -0.18165700 & $\mathrm{H}$ & 5.83961100 & 1.18285900 & -0.51530900 \\
\hline $\mathrm{H}$ & -5.68740300 & 3.33970100 & -0.67640700 & $\mathrm{H}$ & 5.72609100 & 2.19209300 & -1.95213400 \\
\hline $\mathrm{H}$ & -5.39118700 & 5.68281900 & -1.45793300 & $\mathrm{C}$ & 4.06593400 & 0.81669600 & -1.70934500 \\
\hline $\mathrm{H}$ & -3.09887500 & 6.61411700 & -1.72253500 & $\mathrm{H}$ & 3.44885400 & 1.53891800 & -1.16292200 \\
\hline $\mathrm{H}$ & -1.13003500 & 5.22601700 & -1.20473400 & $\mathrm{H}$ & 3.78686600 & 0.89636400 & -2.77208300 \\
\hline $\mathrm{H}$ & -0.83909200 & 2.68853600 & 2.58590000 & $\mathrm{C}$ & 6.18179900 & -1.26261200 & -1.84514200 \\
\hline $\mathrm{H}$ & 0.36649700 & 4.11348100 & 4.19894400 & $\mathrm{H}$ & 6.57100800 & -1.35585300 & -0.82458400 \\
\hline $\mathrm{H}$ & 2.09063600 & 5.72307000 & 3.40379000 & $\mathrm{H}$ & 6.74997000 & -1.97615300 & -2.45706300 \\
\hline $\mathrm{H}$ & 2.58568600 & 5.89594700 & 0.97248000 & $\mathrm{C}$ & 3.72663100 & -0.62008300 & -1.21815600 \\
\hline $\mathrm{H}$ & 1.37533100 & 4.49073500 & -0.64401700 & $\mathrm{C}$ & 4.68769700 & -1.63728700 & -1.88773400 \\
\hline $\mathrm{H}$ & -3.35102100 & -2.09293700 & 1.93889600 & $\mathrm{H}$ & 4.39343800 & -1.70956300 & -2.94524400 \\
\hline $\mathrm{H}$ & -5.64570300 & -2.88986600 & 2.35779400 & $\mathrm{H}$ & 4.53414600 & -2.63857600 & -1.46484900 \\
\hline $\mathrm{H}$ & -6.94842100 & -3.97351200 & 0.53454200 & $\mathrm{C}$ & 6.42839300 & 0.17060900 & -2.33953000 \\
\hline $\mathrm{H}$ & -5.91767300 & -4.26490600 & -1.71035300 & $\mathrm{H}$ & 7.49082600 & 0.42822400 & -2.23805300 \\
\hline $\mathrm{H}$ & -3.61536600 & -3.49683900 & -2.12810800 & $\mathrm{H}$ & 6.19211600 & 0.23384200 & -3.41280100 \\
\hline $\mathrm{H}$ & 1.18576000 & -2.38935000 & 0.50979100 & & & & \\
\hline $\mathrm{H}$ & 2.63676400 & -4.28278800 & 1.12414400 & bpro4 & & & \\
\hline $\mathrm{H}$ & 1.72456100 & -6.60089000 & 1.16022400 & $\mathrm{C}$ & 0.68833500 & -1.22412300 & -0.21667900 \\
\hline $\mathrm{H}$ & -0.65619100 & -6.99610000 & 0.55762700 & $\mathrm{H}$ & 0.53589900 & -1.04598600 & -1.28912900 \\
\hline $\mathrm{H}$ & -2.10703800 & -5.10595500 & -0.07947900 & $\mathrm{H}$ & -0.09489000 & -1.92012100 & 0.09953300 \\
\hline $\mathrm{C}$ & -1.85302200 & 0.22292700 & 1.79962500 & $\mathrm{C}$ & 2.06895700 & -1.86611200 & -0.01131400 \\
\hline $\mathrm{O}$ & -2.33551000 & 0.29310000 & 2.85993700 & $\mathrm{H}$ & 2.12486800 & -2.81017000 & -0.56778800 \\
\hline $\mathrm{H}$ & 2.73502000 & -0.84807800 & -1.62709000 & $\mathrm{H}$ & 2.20107900 & -2.12046900 & 1.05083000 \\
\hline $\mathrm{H}$ & -2.53116100 & 0.23435100 & -0.52117900 & $\mathrm{C}$ & 3.19372800 & -0.92431200 & -0.45675000 \\
\hline $\mathrm{H}$ & 1.11458100 & 0.05732500 & -0.79798900 & $\mathrm{H}$ & 3.12024500 & -0.75703900 & -1.54038700 \\
\hline $\mathrm{C}$ & 4.73911600 & -1.25221000 & 1.14885600 & $\mathrm{H}$ & 4.17692000 & -1.37518100 & -0.27529100 \\
\hline $\mathrm{H}$ & 5.58371800 & -0.54335200 & 1.15576400 & $\mathrm{C}$ & 1.70398900 & 1.03996800 & 0.15883200 \\
\hline $\mathrm{H}$ & 5.13527000 & -2.16579700 & 0.68650800 & $\mathrm{C}$ & 3.09833000 & 0.43118300 & 0.27071600 \\
\hline $\mathrm{C}$ & 3.59570800 & -0.70435800 & 0.30669200 & $\mathrm{H}$ & 3.31188600 & 0.27792200 & 1.33966400 \\
\hline $\mathrm{C}$ & 4.35136700 & -1.56617700 & 2.60017700 & $\mathrm{H}$ & 3.82099800 & 1.15760000 & -0.11246800 \\
\hline
\end{tabular}




\begin{tabular}{|c|c|c|c|c|c|c|c|}
\hline $\mathrm{O}$ & 1.54225200 & 2.18860500 & -0.21235600 & $\mathrm{C}$ & 1.18702300 & 3.03058700 & 0.96625000 \\
\hline $\mathrm{C}$ & -2.26267100 & -0.09753900 & -1.46305500 & $\mathrm{C}$ & -5.18649800 & -0.12572900 & -0.70258700 \\
\hline $\mathrm{H}$ & -1.63404100 & -0.96682500 & -1.69397100 & $\mathrm{C}$ & -6.57827900 & -0.04378800 & -0.68104600 \\
\hline $\mathrm{H}$ & -2.72455200 & 0.20537000 & -2.41205100 & $\mathrm{C}$ & -7.34193400 & -1.13244000 & -0.25300300 \\
\hline $\mathrm{C}$ & -1.40781800 & 1.06517700 & -0.92563100 & $\mathrm{C}$ & -6.70260800 & -2.30378300 & 0.15215500 \\
\hline $\mathrm{H}$ & -0.60267000 & 1.31109300 & -1.62693600 & $\mathrm{C}$ & -5.30869300 & -2.39378200 & 0.11881900 \\
\hline $\mathrm{H}$ & -2.04842700 & 1.95784500 & -0.88509500 & $\mathrm{C}$ & -4.53302500 & -1.30880800 & -0.31682200 \\
\hline $\mathrm{C}$ & -2.72960500 & -0.87940600 & 0.89846800 & $\mathrm{C}$ & -2.54202400 & -4.22189200 & -0.51264900 \\
\hline $\mathrm{H}$ & -2.10895300 & -1.77926800 & 0.78982600 & $\mathrm{C}$ & -2.21735700 & -5.47740000 & 0.00484400 \\
\hline $\mathrm{H}$ & -3.51826400 & -1.13867800 & 1.61708500 & $\mathrm{C}$ & -1.62612400 & -5.58237400 & 1.26610800 \\
\hline $\mathrm{C}$ & -1.89797500 & 0.28863600 & 1.45498100 & $\mathrm{C}$ & -1.35337900 & -4.42691100 & 2.00142200 \\
\hline $\mathrm{H}$ & -2.59502500 & 1.10699800 & 1.68851300 & $\mathrm{C}$ & -1.66136600 & -3.17011800 & 1.47512400 \\
\hline $\mathrm{H}$ & -1.43526800 & 0.00523700 & 2.41054900 & $\mathrm{C}$ & -2.26716800 & -3.05184700 & 0.21533500 \\
\hline $\mathrm{C}$ & -3.34657600 & -0.51980400 & -0.46053800 & $\mathrm{H}$ & 0.56405100 & 3.23086500 & -2.36936400 \\
\hline $\mathrm{H}$ & -3.92408300 & -1.36556200 & -0.85568800 & $\mathrm{H}$ & 1.57029900 & 1.99614000 & -1.61037400 \\
\hline $\mathrm{H}$ & -4.05791800 & 0.30875800 & -0.32372300 & $\mathrm{H}$ & -1.19251800 & 1.52683900 & -2.86247400 \\
\hline $\mathrm{C}$ & 0.54068100 & 0.11464700 & 0.55648000 & $\mathrm{H}$ & 0.38534300 & 0.97721300 & -3.48824000 \\
\hline $\mathrm{H}$ & 0.72872800 & -0.12728400 & 1.61755800 & $\mathrm{H}$ & -2.64022000 & -2.36431000 & -2.69485100 \\
\hline $\mathrm{C}$ & -0.82165500 & 0.85624700 & 0.49500500 & $\mathrm{H}$ & -2.90381300 & -0.61388500 & -2.68080500 \\
\hline \multirow[t]{2}{*}{$\mathrm{H}$} & -0.58087500 & 1.85954600 & 0.86472500 & $\mathrm{H}$ & -0.27693600 & -2.06867200 & -2.04003000 \\
\hline & & & & $\mathrm{H}$ & -0.58947700 & -1.14052300 & -3.51004400 \\
\hline 21 & & & & $\mathrm{H}$ & -0.10828100 & 5.05642400 & -1.79998500 \\
\hline $\mathrm{Ru}$ & -1.41425100 & 0.51739600 & 0.17750100 & $\mathrm{H}$ & -1.43569100 & 7.11774700 & -1.80406500 \\
\hline $\mathrm{P}$ & -0.25470300 & 2.53368600 & -0.09457500 & $\mathrm{H}$ & -3.31616900 & 7.40690600 & -0.19992800 \\
\hline $\mathrm{P}$ & -2.68161200 & -1.35232600 & -0.39196000 & $\mathrm{H}$ & -3.85551600 & 5.57821400 & 1.40276100 \\
\hline $\mathrm{N}$ & -0.30844800 & -0.00230100 & -1.72775500 & $\mathrm{H}$ & -2.55183400 & 3.48697000 & 1.38377300 \\
\hline $\mathrm{C}$ & 0.53497200 & 2.30537700 & -1.78724100 & $\mathrm{H}$ & 1.09190100 & 1.18992400 & 2.06596500 \\
\hline $\mathrm{C}$ & -0.18690900 & 1.20790300 & -2.57559400 & $\mathrm{H}$ & 3.03865800 & 1.74094500 & 3.52558300 \\
\hline $\mathrm{C}$ & -2.32344200 & -1.42432900 & -2.22883700 & $\mathrm{H}$ & 4.23550100 & 3.90255300 & 3.23344800 \\
\hline $\mathrm{C}$ & -0.82349000 & -1.20672900 & -2.43779200 & $\mathrm{H}$ & 3.46796300 & 5.51327000 & 1.50105200 \\
\hline $\mathrm{C}$ & -0.92588700 & 5.15074100 & -1.09139800 & $\mathrm{H}$ & 1.52385000 & 4.97861700 & 0.08360100 \\
\hline $\mathrm{C}$ & -1.67702700 & 6.32889600 & -1.09603400 & $\mathrm{H}$ & -4.59102400 & 0.73454300 & -0.99491400 \\
\hline $\mathrm{C}$ & -2.73117500 & 6.49090300 & -0.19718900 & $\mathrm{H}$ & -7.06591900 & 0.87869300 & -0.98592100 \\
\hline $\mathrm{C}$ & -3.03284100 & 5.46547100 & 0.70159900 & $\mathrm{H}$ & -8.42644100 & -1.06388400 & -0.22649700 \\
\hline $\mathrm{C}$ & -2.28987800 & 4.28506800 & 0.69764700 & $\mathrm{H}$ & -7.28605400 & -3.15312200 & 0.49851000 \\
\hline $\mathrm{C}$ & -1.22012500 & 4.11131500 & -0.19550300 & $\mathrm{H}$ & -4.83143100 & -3.31308500 & 0.43982700 \\
\hline $\mathrm{C}$ & 1.62304800 & 2.13139300 & 1.94964100 & $\mathrm{H}$ & -3.02607200 & -4.16235400 & -1.48382100 \\
\hline $\mathrm{C}$ & 2.71679600 & 2.44421600 & 2.76134500 & $\mathrm{H}$ & -2.43204000 & -6.37228900 & -0.57377100 \\
\hline $\mathrm{C}$ & 3.38657900 & 3.65743200 & 2.60015400 & $\mathrm{H}$ & -1.37971500 & -6.55993700 & 1.67234100 \\
\hline $\mathrm{C}$ & 2.95565900 & 4.56261900 & 1.62658300 & $\mathrm{H}$ & -0.89429100 & -4.50152200 & 2.98379900 \\
\hline $\mathrm{C}$ & 1.86094800 & 4.25401400 & 0.81905600 & $\mathrm{H}$ & -1.41655400 & -2.26650100 & 2.02568100 \\
\hline
\end{tabular}




\begin{tabular}{|c|c|c|c|c|c|c|c|}
\hline C & -2.24744100 & 0.89697100 & 1.78189500 & $\mathrm{Ru}$ & -0.80777700 & 0.57519700 & -0.00892000 \\
\hline $\mathrm{O}$ & -2.76224700 & 1.12983000 & 2.80409200 & $\mathrm{P}$ & -2.45288700 & -0.99621900 & 0.62807300 \\
\hline $\mathrm{H}$ & -0.18470400 & -0.33341800 & 0.99901400 & $\mathrm{P}$ & 0.36619800 & 2.59865900 & 0.15534600 \\
\hline $\mathrm{H}$ & -2.54468700 & 1.32927600 & -0.80013100 & $\mathrm{~N}$ & -0.21752800 & 0.37296100 & 2.14075000 \\
\hline $\mathrm{H}$ & 0.63988600 & -0.23037600 & -1.41903600 & $\mathrm{C}$ & -1.94536600 & -1.35016700 & 2.38386800 \\
\hline C & 2.41268900 & -5.31801800 & 0.67892000 & $\mathrm{C}$ & -1.33758800 & -0.08594800 & 3.00056200 \\
\hline $\mathrm{H}$ & 1.68303900 & -5.40009000 & -0.13963300 & $\mathrm{C}$ & 0.27636600 & 2.83871400 & 2.00716800 \\
\hline $\mathrm{H}$ & 2.03459800 & -5.94973300 & 1.49283600 & $\mathrm{C}$ & 0.56480300 & 1.50856100 & 2.70967800 \\
\hline C & 2.48428700 & -3.85503600 & 1.14371100 & $\mathrm{C}$ & -4.93576900 & -0.27975700 & 1.89317000 \\
\hline $\mathrm{H}$ & 1.48799800 & -3.50465000 & 1.43840400 & $\mathrm{C}$ & -6.23158500 & 0.24655000 & 1.88672800 \\
\hline $\mathrm{H}$ & 3.12194400 & -3.79047100 & 2.03950600 & $\mathrm{C}$ & -6.78066400 & 0.74443300 & 0.70642600 \\
\hline C & 3.77946800 & -5.82671200 & 0.19727200 & $\mathrm{C}$ & -6.02456700 & 0.72115200 & -0.46932100 \\
\hline $\mathrm{H}$ & 3.69465600 & -6.85513600 & -0.17696200 & $\mathrm{C}$ & -4.72925400 & 0.20837300 & -0.45976700 \\
\hline $\mathrm{H}$ & 4.47479300 & -5.86035700 & 1.04953900 & $\mathrm{C}$ & -4.16788300 & -0.30579000 & 0.72149900 \\
\hline C & 4.35947000 & -4.91064800 & -0.89039700 & $\mathrm{C}$ & -2.17644400 & -3.00962000 & -1.31555500 \\
\hline $\mathrm{H}$ & 3.72614700 & -4.96923100 & -1.78804900 & $\mathrm{C}$ & -2.47040700 & -4.23534300 & -1.91919600 \\
\hline $\mathrm{H}$ & 5.35696800 & -5.25649300 & -1.19058500 & $\mathrm{C}$ & -3.37212000 & -5.11348900 & -1.31956000 \\
\hline C & 4.43115000 & -3.44855000 & -0.42089200 & $\mathrm{C}$ & -3.98711600 & -4.76524000 & -0.11291300 \\
\hline $\mathrm{H}$ & 5.15151800 & -3.36981900 & 0.40846100 & $\mathrm{C}$ & -3.70488500 & -3.53826600 & 0.48380400 \\
\hline $\mathrm{H}$ & 4.82085000 & -2.81725700 & -1.22987400 & $\mathrm{C}$ & -2.79239600 & -2.65018300 & -0.11111100 \\
\hline C & 7.03516900 & 0.66915900 & 0.33536500 & $\mathrm{C}$ & -1.69333400 & 4.17585900 & -0.97767200 \\
\hline C & 5.80003900 & 0.02871100 & 0.43831500 & $\mathrm{C}$ & -2.26955400 & 5.36103200 & -1.44033100 \\
\hline C & 4.84112000 & 0.16183300 & -0.57916200 & $\mathrm{C}$ & -1.53658200 & 6.54811200 & -1.43690700 \\
\hline C & 5.15115800 & 0.95111800 & -1.70180600 & $\mathrm{C}$ & -0.21991200 & 6.54649100 & -0.97069800 \\
\hline C & 6.38323900 & 1.58707100 & -1.80543400 & $\mathrm{C}$ & 0.35919100 & 5.36345900 & -0.51199400 \\
\hline C & 7.32954700 & 1.44787100 & -0.78468100 & $\mathrm{C}$ & -0.37307600 & 4.16407100 & -0.50403700 \\
\hline $\mathrm{H}$ & 7.76648700 & 0.56023300 & 1.13136400 & $\mathrm{C}$ & 3.13426000 & 3.32170700 & 0.53397800 \\
\hline $\mathrm{H}$ & 5.59087600 & -0.56931900 & 1.31791200 & $\mathrm{C}$ & 4.44981400 & 3.47039700 & 0.08467900 \\
\hline $\mathrm{H}$ & 4.40558100 & 1.04572200 & -2.48384300 & $\mathrm{C}$ & 4.78980600 & 3.11333100 & -1.22013700 \\
\hline $\mathrm{H}$ & 6.61011200 & 2.19159700 & -2.67935900 & $\mathrm{C}$ & 3.81073700 & 2.59659000 & -2.07328800 \\
\hline $\mathrm{H}$ & 8.29252700 & 1.94545000 & -0.86322700 & $\mathrm{C}$ & 2.50209800 & 2.43646100 & -1.62095600 \\
\hline C & 3.49673800 & -0.49654100 & -0.53684000 & $\mathrm{C}$ & 2.14252300 & 2.80665900 & -0.31387900 \\
\hline o & 2.68780500 & -0.27106300 & -1.43936000 & $\mathrm{H}$ & -2.75291700 & -1.75022500 & 3.00495600 \\
\hline C & 3.11122800 & -1.46636500 & 0.56499700 & $\mathrm{H}$ & -1.16197000 & -2.11344700 & 2.30756800 \\
\hline $\mathrm{H}$ & 2.10533500 & -1.17447200 & 0.89460300 & $\mathrm{H}$ & -2.07797900 & 0.71695200 & 3.08061500 \\
\hline $\mathrm{H}$ & 3.77767600 & -1.40458000 & 1.43022400 & $\mathrm{H}$ & -0.96559700 & -0.31095800 & 4.01181400 \\
\hline C & 3.06030500 & -2.92981900 & 0.05594300 & $\mathrm{H}$ & 0.93807900 & 3.63058600 & 2.37671400 \\
\hline \multirow[t]{2}{*}{$\mathrm{H}$} & 2.37350700 & -2.94478500 & -0.80357300 & $\mathrm{H}$ & -0.75351300 & 3.15789600 & 2.19787500 \\
\hline & & & & $\mathrm{H}$ & 1.62535400 & 1.25652100 & 2.60184400 \\
\hline \multicolumn{4}{|c|}{ TS30 } & $\mathrm{H}$ & 0.36579100 & 1.59941300 & 3.78612300 \\
\hline \multicolumn{4}{|c|}{ imaginary frequency $=-443.02 \mathrm{~cm}^{-1}$} & $\mathrm{H}$ & -4.53492300 & -0.66287000 & 2.82652800 \\
\hline
\end{tabular}




\begin{tabular}{|c|c|c|c|c|c|c|c|}
\hline $\mathrm{H}$ & -6.80865200 & 0.26368800 & 2.80780000 & $\mathrm{C}$ & 1.35979600 & -2.80474400 & -0.58872100 \\
\hline $\mathrm{H}$ & -7.78845800 & 1.15096800 & 0.70070000 & $\mathrm{C}$ & 1.07888200 & -4.08374800 & -0.09113800 \\
\hline $\mathrm{H}$ & -6.44243800 & 1.10952700 & -1.39442300 & $\mathrm{C}$ & 0.96981800 & -5.17600200 & -0.95368600 \\
\hline $\mathrm{H}$ & -4.14401400 & 0.21587400 & -1.37526100 & $\mathrm{C}$ & 1.12894800 & -5.00174400 & -2.32955500 \\
\hline $\mathrm{H}$ & -1.45987200 & -2.33714800 & -1.77525400 & $\mathrm{H}$ & 1.50252500 & -3.57736700 & -3.90929200 \\
\hline $\mathrm{H}$ & -1.98066800 & -4.50287400 & -2.85041100 & $\mathrm{H}$ & 1.68928000 & -1.64500200 & -2.37742500 \\
\hline $\mathrm{H}$ & -3.59837700 & -6.06758800 & -1.78863400 & $\mathrm{H}$ & 0.96848300 & -4.20230200 & 0.98173300 \\
\hline $\mathrm{H}$ & -4.69249200 & -5.44577400 & 0.35704900 & $\mathrm{H}$ & 0.76161300 & -6.16399300 & -0.55044800 \\
\hline $\mathrm{H}$ & -4.21069900 & -3.26487600 & 1.40623400 & $\mathrm{H}$ & 1.05003700 & -5.85189700 & -3.00268900 \\
\hline $\mathrm{H}$ & -2.26507600 & 3.25430000 & -0.97644300 & $\mathrm{C}$ & 1.58817900 & -1.67945000 & 0.40905900 \\
\hline $\mathrm{H}$ & -3.29234600 & 5.35179200 & -1.80772300 & $\mathrm{O}$ & 1.24700800 & -1.88101500 & 1.61684400 \\
\hline $\mathrm{H}$ & -1.98535300 & 7.46910900 & -1.79985400 & $\mathrm{C}$ & 2.85028300 & -0.81860100 & 0.18773800 \\
\hline $\mathrm{H}$ & 0.36016000 & 7.46570400 & -0.96890200 & $\mathrm{H}$ & 2.66542500 & 0.15382000 & 0.6521230 \\
\hline $\mathrm{H}$ & 1.38952100 & 5.37508800 & -0.16942600 & $\mathrm{H}$ & 3.02737900 & -0.62783000 & -0.87637500 \\
\hline $\mathrm{H}$ & 2.89357500 & 3.61499300 & 1.55148300 & $\mathrm{C}$ & 4.11321700 & -1.42821700 & 0.84376000 \\
\hline $\mathrm{H}$ & 5.20602000 & 3.86767900 & 0.75665000 & $\mathrm{H}$ & 3.82608200 & -1.72346200 & 1.86383800 \\
\hline $\mathrm{H}$ & 5.81172200 & 3.23224900 & -1.56973900 & & & & \\
\hline $\mathrm{H}$ & 4.06852000 & 2.31092200 & -3.08959300 & 22 & & & \\
\hline $\mathrm{H}$ & 1.75095400 & 2.01474500 & -2.28399400 & $\mathrm{Ru}$ & 0.81407400 & 0.70507200 & -0.01067300 \\
\hline $\mathrm{C}$ & -1.21324300 & 0.64775900 & -1.81693400 & $\mathrm{P}$ & 2.43740100 & -0.93506600 & -0.61367500 \\
\hline $\mathrm{O}$ & -1.46051500 & 0.68371400 & -2.95643100 & $\mathrm{P}$ & -0.45035800 & 2.68335700 & -0.20782800 \\
\hline $\mathrm{H}$ & 0.53667600 & -0.62456600 & -0.23152700 & $\mathrm{~N}$ & 0.23642000 & 0.44021500 & -2.11943900 \\
\hline $\mathrm{H}$ & -2.01354000 & 1.61354200 & 0.40646700 & $\mathrm{C}$ & 1.98684000 & -1.24973000 & -2.39247800 \\
\hline $\mathrm{H}$ & 0.43040400 & -0.44208900 & 2.06688800 & $\mathrm{C}$ & 1.36430700 & 0.01777600 & -2.98485400 \\
\hline $\mathrm{C}$ & 6.49542900 & -0.90507500 & 1.62148400 & $\mathrm{C}$ & -0.40038100 & 2.87333300 & -2.06300500 \\
\hline $\mathrm{H}$ & 6.27150700 & -1.16386100 & 2.66734300 & $\mathrm{C}$ & -0.61898400 & 1.50273200 & -2.71332600 \\
\hline $\mathrm{H}$ & 7.26820500 & -0.12511200 & 1.65224100 & $\mathrm{C}$ & 4.62166700 & 0.75109200 & 0.06417900 \\
\hline $\mathrm{C}$ & 5.22285100 & -0.36204200 & 0.95314300 & $\mathrm{C}$ & 5.95946900 & 1.15403300 & 0.04894900 \\
\hline $\mathrm{H}$ & 4.84857800 & 0.50801300 & 1.50898900 & $\mathrm{C}$ & 6.89674900 & 0.44748600 & -0.70419200 \\
\hline $\mathrm{H}$ & 5.47017500 & 0.00413900 & -0.05516700 & $\mathrm{C}$ & 6.49345700 & -0.67384900 & -1.43376900 \\
\hline $\mathrm{C}$ & 7.02275100 & -2.15245000 & 0.89767100 & $\mathrm{C}$ & 5.16028100 & -1.08094900 & -1.40953300 \\
\hline $\mathrm{H}$ & 7.90286800 & -2.55338200 & 1.41772800 & $\mathrm{C}$ & 4.20297400 & -0.36700300 & -0.66932200 \\
\hline $\mathrm{H}$ & 7.35704400 & -1.86988700 & -0.11210800 & $\mathrm{C}$ & 2.58971800 & -2.71493200 & 1.53642700 \\
\hline $\mathrm{C}$ & 5.92976100 & -3.22462600 & 0.78608300 & $\mathrm{C}$ & 2.82165500 & -3.94324000 & 2.15481600 \\
\hline $\mathrm{H}$ & 5.68010300 & -3.59016400 & 1.79349100 & $\mathrm{C}$ & 3.08702300 & -5.07701400 & 1.38514500 \\
\hline $\mathrm{H}$ & 6.30219600 & -4.09179700 & 0.22499100 & $\mathrm{C}$ & 3.11242500 & -4.98000900 & -0.00684400 \\
\hline $\mathrm{C}$ & 4.65653000 & -2.67688800 & 0.12009000 & $\mathrm{C}$ & 2.88404100 & -3.75141100 & -0.62762000 \\
\hline $\mathrm{H}$ & 4.87913000 & -2.41401700 & -0.92609600 & $\mathrm{C}$ & 2.62830600 & -2.60410000 & 0.13880800 \\
\hline $\mathrm{H}$ & 3.89369100 & -3.46098700 & 0.08464800 & $\mathrm{C}$ & 1.52990600 & 4.37592300 & 0.89956000 \\
\hline $\mathrm{C}$ & 1.38806000 & -3.72440700 & -2.83803900 & $\mathrm{C}$ & 2.05072500 & 5.59876600 & 1.32858900 \\
\hline $\mathrm{C}$ & 1.50448700 & -2.63651500 & -1.97369800 & $\mathrm{C}$ & 1.26924900 & 6.75303600 & 1.2723690 \\
\hline
\end{tabular}




\begin{tabular}{|c|c|c|c|c|c|c|c|}
\hline $\mathrm{C}$ & -0.03911500 & 6.68087100 & 0.78794900 & $\mathrm{H}$ & -0.38970200 & -0.45405900 & -2.00403800 \\
\hline $\mathrm{C}$ & -0.56217500 & 5.46005700 & 0.36295300 & $\mathrm{C}$ & -6.28171100 & -1.49679300 & -1.82163300 \\
\hline $\mathrm{C}$ & 0.21934700 & 4.29303700 & 0.40814700 & $\mathrm{H}$ & -5.93766900 & -1.95760800 & -2.75978300 \\
\hline $\mathrm{C}$ & -3.26321400 & 3.17087900 & -0.55833200 & $\mathrm{H}$ & -7.08938100 & -0.80305600 & -2.09298800 \\
\hline $\mathrm{C}$ & -4.57897200 & 3.23502100 & -0.09135200 & $\mathrm{C}$ & -5.11363800 & -0.72904600 & -1.18284400 \\
\hline $\mathrm{C}$ & -4.86759600 & 2.92411000 & 1.23739900 & $\mathrm{H}$ & -4.73130200 & 0.02214500 & -1.88786300 \\
\hline $\mathrm{C}$ & -3.83622900 & 2.54120900 & 2.09920900 & $\mathrm{H}$ & -5.48112400 & -0.16981700 & -0.30778200 \\
\hline $\mathrm{C}$ & -2.52582300 & 2.46538900 & 1.63120400 & $\mathrm{C}$ & -6.81180200 & -2.59574800 & -0.88929900 \\
\hline $\mathrm{C}$ & -2.22080000 & 2.78709600 & 0.29771300 & $\mathrm{H}$ & -7.61128000 & -3.16400200 & -1.38358900 \\
\hline $\mathrm{H}$ & 2.83800600 & -1.58520400 & -2.99286300 & $\mathrm{H}$ & -7.26296300 & -2.12880000 & -0.00041200 \\
\hline $\mathrm{H}$ & 1.21710400 & -2.02942200 & -2.37079300 & $\mathrm{C}$ & -5.67900400 & -3.53251700 & -0.44646200 \\
\hline $\mathrm{H}$ & 2.10016300 & 0.82627300 & -3.06446200 & $\mathrm{H}$ & -5.30719200 & -4.08649500 & -1.32147900 \\
\hline $\mathrm{H}$ & 0.99438800 & -0.20338700 & -3.99746500 & $\mathrm{H}$ & -6.05996700 & -4.28416200 & 0.25783400 \\
\hline $\mathrm{H}$ & -1.11026300 & 3.61792300 & -2.44172800 & $\mathrm{C}$ & -4.51427300 & -2.75668600 & 0.19027100 \\
\hline $\mathrm{H}$ & 0.60763500 & 3.24319900 & -2.28148300 & $\mathrm{H}$ & -4.86417500 & -2.28540000 & 1.12295000 \\
\hline $\mathrm{H}$ & -1.66088900 & 1.18908300 & -2.58136100 & $\mathrm{H}$ & -3.71875500 & -3.45169100 & 0.47412300 \\
\hline $\mathrm{H}$ & -0.44140800 & 1.57137000 & -3.79499700 & $\mathrm{C}$ & -1.08997500 & -3.65405300 & 2.85717700 \\
\hline $\mathrm{H}$ & 3.89532400 & 1.30791900 & 0.64552700 & $\mathrm{C}$ & -1.22489700 & -2.54263300 & 2.02527100 \\
\hline $\mathrm{H}$ & 6.26555600 & 2.02260300 & 0.62613800 & $\mathrm{C}$ & -1.16470100 & -2.67654500 & 0.63122000 \\
\hline $\mathrm{H}$ & 7.93642800 & 0.76350000 & -0.72021200 & $\mathrm{C}$ & -0.94331900 & -3.94608700 & 0.08806200 \\
\hline $\mathrm{H}$ & 7.21823400 & -1.23534800 & -2.01740600 & $\mathrm{C}$ & -0.81065500 & -5.06253200 & 0.91752600 \\
\hline $\mathrm{H}$ & 4.87205600 & -1.97130600 & -1.96132700 & $\mathrm{C}$ & -0.88836700 & -4.92233400 & 2.30434000 \\
\hline $\mathrm{H}$ & 2.36877300 & -1.84183500 & 2.14338600 & $\mathrm{H}$ & -1.14335200 & -3.53210200 & 3.93675900 \\
\hline $\mathrm{H}$ & 2.78226900 & -4.01523400 & 3.23796600 & $\mathrm{H}$ & -1.37823200 & -1.55853100 & 2.46399800 \\
\hline $\mathrm{H}$ & 3.26306500 & -6.03460800 & 1.86777500 & $\mathrm{H}$ & -0.89213100 & -4.03210600 & -0.99234900 \\
\hline $\mathrm{H}$ & 3.30586100 & -5.86146100 & -0.61263700 & $\mathrm{H}$ & -0.64651500 & -6.04448400 & 0.47938900 \\
\hline $\mathrm{H}$ & 2.90050100 & -3.69703200 & -1.71207400 & $\mathrm{H}$ & -0.78840900 & -5.79120200 & 2.95047200 \\
\hline $\mathrm{H}$ & 2.13838500 & 3.47953400 & 0.94507100 & $\mathrm{C}$ & -1.39507500 & -1.48592300 & -0.31132400 \\
\hline $\mathrm{H}$ & 3.06659300 & 5.64522500 & 1.71182100 & $\mathrm{O}$ & -1.09610900 & -1.71315500 & -1.58811000 \\
\hline $\mathrm{H}$ & 1.67399500 & 7.70373100 & 1.60913200 & $\mathrm{C}$ & -2.80752400 & -0.84260100 & -0.12239400 \\
\hline $\mathrm{H}$ & -0.65590900 & 7.57471700 & 0.74623300 & $\mathrm{H}$ & -2.76584700 & 0.12971800 & -0.62666800 \\
\hline $\mathrm{H}$ & -1.58693500 & 5.41494000 & 0.00604300 & $\mathrm{H}$ & -3.00986900 & -0.63166500 & 0.93652300 \\
\hline $\mathrm{H}$ & -3.06366400 & 3.41890000 & -1.59632200 & $\mathrm{C}$ & -3.96267100 & -1.65877500 & -0.74282800 \\
\hline $\mathrm{H}$ & -5.37628900 & 3.52699100 & -0.76944200 & $\mathrm{H}$ & -3.55761000 & -2.13784100 & -1.64554900 \\
\hline $\mathrm{H}$ & -5.89078700 & 2.97447300 & 1.59935100 & & & & \\
\hline $\mathrm{H}$ & -4.05408100 & 2.29162900 & 3.13401200 & 23 & & & \\
\hline $\mathrm{H}$ & -1.73299800 & 2.14744800 & 2.30355800 & $\mathrm{Ru}$ & -0.33862200 & 0.84196100 & 0.07844800 \\
\hline $\mathrm{C}$ & 1.22971700 & 0.83181400 & 1.80316300 & $\mathrm{P}$ & -2.56219300 & 0.18960000 & -0.42005700 \\
\hline $\mathrm{O}$ & 1.48642800 & 0.90987600 & 2.93658100 & $\mathrm{P}$ & 1.84855100 & 1.68466400 & -0.17402900 \\
\hline $\mathrm{H}$ & -0.66179700 & -0.62992400 & 0.16943400 & $\mathrm{~N}$ & -0.05402500 & 0.35242600 & -2.08222100 \\
\hline $\mathrm{H}$ & 1.98203400 & 1.72877700 & -0.38414600 & $\mathrm{C}$ & -2.32112200 & -0.58440600 & -2.10032100 \\
\hline
\end{tabular}




\begin{tabular}{|c|c|c|c|c|c|c|c|}
\hline $\mathrm{C}$ & -1.29076100 & 0.24025600 & -2.87919900 & $\mathrm{H}$ & -4.35785100 & 4.83631800 & -0.76846500 \\
\hline $\mathrm{C}$ & 2.29674300 & 1.03533000 & -1.86802900 & $\mathrm{H}$ & -6.63706600 & 4.11366400 & -1.45435200 \\
\hline $\mathrm{C}$ & 1.05673400 & 1.03552500 & -2.76785500 & $\mathrm{H}$ & -7.15131000 & 1.68793000 & -1.66356300 \\
\hline $\mathrm{C}$ & -3.77100400 & -0.74840900 & 1.90715700 & $\mathrm{H}$ & -5.41193800 & 0.00636500 & -1.18448500 \\
\hline $\mathrm{C}$ & -4.47110400 & -1.64608300 & 2.71164100 & $\mathrm{H}$ & 2.14981700 & 0.03078300 & 2.2098320 \\
\hline $\mathrm{C}$ & -4.88645100 & -2.87600300 & 2.19376300 & $\mathrm{H}$ & 4.06937300 & -0.57329700 & 3.63657700 \\
\hline $\mathrm{C}$ & -4.59921400 & -3.19995300 & 0.86810500 & $\mathrm{H}$ & 6.34525500 & 0.29511800 & 3.10557600 \\
\hline $\mathrm{C}$ & -3.90309300 & -2.29852700 & 0.05818700 & $\mathrm{H}$ & 6.67115300 & 1.78046600 & $1.1377570 \mathrm{C}$ \\
\hline $\mathrm{C}$ & -3.48335100 & -1.06166200 & 0.56751400 & $\mathrm{H}$ & 4.74634100 & 2.41285600 & -0.27113900 \\
\hline $\mathrm{C}$ & -3.59681800 & 2.82867000 & -0.60837900 & $\mathrm{H}$ & 3.11006800 & 3.64008100 & -2.14403200 \\
\hline $\mathrm{C}$ & -4.58842400 & 3.77896700 & -0.86927000 & $\mathrm{H}$ & 3.25624600 & 6.09034900 & -2.15409600 \\
\hline $\mathrm{C}$ & -5.86644000 & 3.37408400 & -1.25305200 & $\mathrm{H}$ & 2.24435500 & 7.41828800 & -0.31038100 \\
\hline $\mathrm{C}$ & -6.15544300 & 2.01144900 & -1.37201100 & $\mathrm{H}$ & 1.06007800 & 6.24927800 & $1.5421350 \mathrm{C}$ \\
\hline $\mathrm{C}$ & -5.16876600 & 1.06305500 & -1.10872500 & $\mathrm{H}$ & 0.86807900 & 3.78838600 & 1.53435000 \\
\hline $\mathrm{C}$ & -3.87431300 & 1.46071900 & -0.72926200 & $\mathrm{C}$ & -0.57370800 & 1.21362800 & 1.87859000 \\
\hline $\mathrm{C}$ & 3.13522700 & 0.41936700 & 1.97526900 & $\mathrm{O}$ & -0.74087600 & 1.49815400 & 2.99659600 \\
\hline $\mathrm{C}$ & 4.22517600 & 0.07632900 & 2.78017400 & $\mathrm{H}$ & -0.74969200 & 2.37072900 & -0.20941800 \\
\hline $\mathrm{C}$ & 5.49759900 & 0.56125000 & 2.47943500 & $\mathrm{C}$ & 1.31281800 & -4.90293500 & -3.99797500 \\
\hline $\mathrm{C}$ & 5.68196400 & 1.39592700 & 1.37251300 & $\mathrm{H}$ & 0.69420800 & -4.14971600 & -4.50971300 \\
\hline $\mathrm{C}$ & 4.59538600 & 1.74393400 & 0.57204300 & $\mathrm{H}$ & 1.05005600 & -5.87276200 & -4.44086200 \\
\hline $\mathrm{C}$ & 3.31142100 & 1.25230800 & 0.86283900 & $\mathrm{C}$ & 0.97856300 & -4.89494400 & -2.49773600 \\
\hline $\mathrm{C}$ & 2.65486500 & 4.19406000 & -1.32866800 & $\mathrm{H}$ & -0.09301300 & -5.08494900 & -2.34896000 \\
\hline $\mathrm{C}$ & 2.74243800 & 5.58945000 & -1.33764900 & $\mathrm{H}$ & 1.50976800 & -5.72396600 & -2.00441700 \\
\hline $\mathrm{C}$ & 2.17449700 & 6.33384200 & -0.30469400 & $\mathrm{C}$ & 2.79536900 & -4.58799400 & -4.24771900 \\
\hline $\mathrm{C}$ & 1.50996400 & 5.67743400 & 0.73493300 & $\mathrm{H}$ & 3.00244600 & -4.54748900 & -5.32531200 \\
\hline $\mathrm{C}$ & 1.41000900 & 4.28735500 & 0.73638000 & $\mathrm{H}$ & 3.41104700 & -5.40432500 & -3.84132200 \\
\hline $\mathrm{C}$ & 1.98870800 & 3.52475200 & -0.29227300 & $\mathrm{C}$ & 3.20290200 & -3.26947800 & -3.57350200 \\
\hline $\mathrm{H}$ & -1.91305800 & -1.58440200 & -1.91939200 & $\mathrm{H}$ & 2.67481000 & -2.43703200 & -4.06449300 \\
\hline $\mathrm{H}$ & -3.25860800 & -0.66995800 & -2.65942700 & $\mathrm{H}$ & 4.27565700 & -3.08400700 & -3.71737000 \\
\hline $\mathrm{H}$ & -1.08303900 & -0.24027500 & -3.84839900 & $\mathrm{C}$ & 2.86043200 & -3.27337800 & -2.07471000 \\
\hline $\mathrm{H}$ & -1.66843000 & 1.24907500 & -3.08331600 & $\mathrm{H}$ & 3.46749200 & -4.04052300 & -1.56884500 \\
\hline $\mathrm{H}$ & 3.12646200 & 1.57191700 & -2.33890900 & $\mathrm{H}$ & 3.14644000 & -2.31488600 & -1.62262000 \\
\hline $\mathrm{H}$ & 2.63368000 & 0.00523900 & -1.70340800 & $\mathrm{C}$ & 1.78185700 & -3.16847700 & 4.05507600 \\
\hline $\mathrm{H}$ & 0.74292900 & 2.06140400 & -2.99353000 & $\mathrm{C}$ & 1.94476400 & -2.88788000 & 2.69728100 \\
\hline $\mathrm{H}$ & 1.28624400 & 0.53962000 & -3.72452400 & $\mathrm{C}$ & 0.86200200 & -2.47020900 & 1.90851400 \\
\hline $\mathrm{H}$ & -3.43691700 & 0.19668600 & 2.32698700 & $\mathrm{C}$ & -0.39087300 & -2.34284800 & 2.51593800 \\
\hline $\mathrm{H}$ & -4.68627300 & -1.38743200 & 3.74488900 & $\mathrm{C}$ & -0.55973300 & -2.62115500 & 3.87468300 \\
\hline $\mathrm{H}$ & -5.42606000 & -3.57880800 & 2.82271900 & $\mathrm{C}$ & 0.52453300 & -3.03415000 & 4.65094700 \\
\hline $\mathrm{H}$ & -4.91341900 & -4.15671800 & 0.45948700 & $\mathrm{H}$ & 2.63483000 & -3.49098300 & 4.64849200 \\
\hline $\mathrm{H}$ & -3.68875200 & -2.57230500 & -0.96978700 & $\mathrm{H}$ & 2.92810500 & -2.99065500 & 2.24069700 \\
\hline $\mathrm{H}$ & -2.60132100 & 3.14090700 & -0.30902400 & $\mathrm{H}$ & -1.22750900 & -2.01914300 & 1.90863600 \\
\hline
\end{tabular}




\begin{tabular}{|c|c|c|c|c|c|c|c|}
\hline $\mathrm{H}$ & -1.54340500 & -2.51298100 & 4.32587400 & $\mathrm{C}$ & -4.37277900 & 3.47623300 & -0.00807000 \\
\hline $\mathrm{H}$ & 0.39418200 & -3.24729900 & 5.70913200 & $\mathrm{C}$ & -4.64781300 & 3.19455000 & 1.33011100 \\
\hline $\mathrm{C}$ & 1.02120200 & -3.59350700 & -0.31492000 & $\mathrm{C}$ & -3.61886900 & 2.76821200 & 2.17419800 \\
\hline $\mathrm{H}$ & 0.00675100 & -3.99639000 & -0.18713000 & $\mathrm{C}$ & -2.32444200 & 2.62074400 & 1.67942200 \\
\hline $\mathrm{H}$ & 1.70236800 & -4.28724100 & 0.19955400 & $\mathrm{C}$ & -2.03271900 & 2.91220800 & 0.33596300 \\
\hline $\mathrm{C}$ & 1.37061000 & -3.57034500 & -1.81207900 & $\mathrm{H}$ & 2.70104200 & -1.79685400 & -2.96402300 \\
\hline $\mathrm{H}$ & 0.77607300 & -2.77047800 & -2.28074000 & $\mathrm{H}$ & 1.07709400 & -2.14590100 & -2.30099100 \\
\hline $\mathrm{O}$ & 0.07833200 & -1.38576200 & -0.15370500 & $\mathrm{H}$ & 2.10031300 & 0.63274400 & -3.09910200 \\
\hline $\mathrm{H}$ & 0.22886200 & -0.59708800 & -1.75400900 & $\mathrm{H}$ & 0.88877800 & -0.34796800 & -3.94541000 \\
\hline $\mathrm{C}$ & 1.04045800 & -2.21263700 & 0.40747400 & $\mathrm{H}$ & -0.94352800 & 3.63385400 & -2.44564100 \\
\hline $\mathrm{H}$ & 2.06328900 & -1.79297700 & 0.29219400 & $\mathrm{H}$ & 0.75796000 & 3.17685100 & -2.31184300 \\
\hline & & & & $\mathrm{H}$ & -1.61495700 & 1.23321700 & -2.52020800 \\
\hline TS & & & & $\mathrm{H}$ & -0.39496800 & 1.52091100 & -3.75783100 \\
\hline $\mathrm{im}$ & uency $=-6$ & $2.32 \mathrm{~cm}^{-1}$ & & $\mathrm{H}$ & 3.99386800 & 1.06017700 & 0.66609200 \\
\hline $\mathrm{Ru}$ & 0.87465300 & 0.67024000 & 0.00502500 & $\mathrm{H}$ & 6.39568300 & 1.65246700 & 0.59590600 \\
\hline $\mathrm{P}$ & 2.38752400 & -1.07290000 & -0.59348300 & $\mathrm{H}$ & 7.96256600 & 0.34102500 & -0.82381500 \\
\hline $\mathrm{P}$ & -0.28236300 & 2.71296600 & -0.20791400 & $\mathrm{H}$ & 7.10691300 & -1.58654000 & -2.14501300 \\
\hline $\mathrm{N}$ & 0.24922000 & 0.39850500 & -2.06379300 & $\mathrm{H}$ & 4.72841000 & -2.20062800 & -2.04288900 \\
\hline $\mathrm{C}$ & 1.88218000 & -1.40522600 & -2.35284000 & $\mathrm{H}$ & 2.28355200 & -1.90658400 & 2.18897800 \\
\hline $\mathrm{C}$ & 1.31139000 & -0.11742400 & -2.95515800 & $\mathrm{H}$ & 2.59232600 & -4.07046100 & 3.33647600 \\
\hline $\mathrm{C}$ & -0.26271300 & 2.86315400 & -2.06496300 & $\mathrm{H}$ & 2.97435100 & -6.14322800 & 2.01587100 \\
\hline $\mathrm{C}$ & -0.55769900 & 1.48547400 & -2.67192800 & $\mathrm{H}$ & 3.02836200 & -6.03182300 & -0.46779900 \\
\hline $\mathrm{C}$ & 4.67376600 & 0.48072700 & 0.05146400 & $\mathrm{H}$ & 2.72888600 & -3.87695300 & -1.61964600 \\
\hline $\mathrm{C}$ & 6.02969800 & 0.81452000 & 0.00843500 & $\mathrm{H}$ & 2.35995900 & 3.38235200 & 0.90611100 \\
\hline $\mathrm{C}$ & 6.90868400 & 0.07851600 & -0.78585700 & $\mathrm{H}$ & 3.41766600 & 5.50656300 & 1.61750400 \\
\hline $\mathrm{C}$ & 6.42823100 & -1.00275000 & -1.52874500 & $\mathrm{H}$ & 2.14255500 & 7.63826300 & 1.48698400 \\
\hline $\mathrm{C}$ & 5.07652900 & -1.34097900 & -1.47722100 & $\mathrm{H}$ & -0.20054400 & 7.62468400 & 0.65074100 \\
\hline $\mathrm{C}$ & 4.17770700 & -0.59671800 & -0.69506900 & $\mathrm{H}$ & -1.26029700 & 5.50687100 & -0.03641000 \\
\hline $\mathrm{C}$ & 2.46090600 & -2.80394800 & 1.60346000 & $\mathrm{H}$ & -2.88302600 & 3.56453400 & -1.54736500 \\
\hline $\mathrm{C}$ & 2.63385300 & -4.02679300 & 2.25178100 & $\mathrm{H}$ & -5.16884200 & 3.80144600 & -0.67235700 \\
\hline $\mathrm{C}$ & 2.84468300 & -5.19016600 & 1.51015400 & $\mathrm{H}$ & -5.65898000 & 3.30114600 & 1.71307600 \\
\hline $\mathrm{C}$ & 2.87629500 & -5.12784000 & 0.11616400 & $\mathrm{H}$ & -3.82653500 & 2.54124000 & 3.21629200 \\
\hline $\mathrm{C}$ & 2.70751300 & -3.90513200 & -0.53423000 & $\mathrm{H}$ & -1.53398500 & 2.27106800 & 2.33872500 \\
\hline $\mathrm{C}$ & 2.50501000 & -2.72876300 & 0.20365600 & $\mathrm{C}$ & 1.31502900 & 0.79919400 & 1.81841800 \\
\hline $\mathrm{C}$ & 1.80248900 & 4.31065300 & 0.84700300 & $\mathrm{O}$ & 1.58781400 & 0.88200400 & 2.94802400 \\
\hline $\mathrm{C}$ & 2.39650200 & 5.51020900 & 1.24573100 & $\mathrm{H}$ & -0.77096300 & -0.60772900 & 0.22990100 \\
\hline $\mathrm{C}$ & 1.68091400 & 6.70557700 & 1.17388800 & $\mathrm{H}$ & 2.09343800 & 1.61000100 & -0.37301800 \\
\hline $\mathrm{C}$ & 0.36512000 & 6.69824500 & 0.70435900 & $\mathrm{H}$ & -0.49838200 & -0.52439000 & -1.90164400 \\
\hline $\mathrm{C}$ & -0.23071100 & 5.50097500 & 0.30940900 & $\mathrm{C}$ & -6.40085600 & -1.10629100 & -1.79319400 \\
\hline $\mathrm{C}$ & 0.48402700 & 4.29262300 & 0.37047500 & $\mathrm{H}$ & -6.07105300 & -1.54518900 & -2.74684500 \\
\hline $\mathrm{C}$ & -3.07268700 & 3.33950500 & -0.50239700 & $\mathrm{H}$ & -7.17106200 & -0.36331100 & -2.04202800 \\
\hline
\end{tabular}




\begin{tabular}{|c|c|c|c|c|c|c|c|}
\hline $\mathrm{C}$ & -5.20276400 & -0.42344600 & -1.11542900 & $\mathrm{C}$ & -3.78636500 & 0.12502100 & -1.30274500 \\
\hline $\mathrm{H}$ & -4.77673500 & 0.33495600 & -1.78663300 & $\mathrm{H}$ & -3.91137500 & 1.21754600 & -1.27581000 \\
\hline $\mathrm{H}$ & -5.55033000 & 0.11827800 & -0.22134700 & $\mathrm{H}$ & -4.13555100 & -0.20347800 & -2.29015600 \\
\hline $\mathrm{C}$ & -6.99300000 & -2.21451600 & -0.91027600 & $\mathrm{C}$ & -2.29622300 & -0.21405800 & -1.13338200 \\
\hline $\mathrm{H}$ & -7.81395800 & -2.72291700 & -1.43363700 & $\mathrm{H}$ & -2.15276500 & -1.29730100 & -1.27424700 \\
\hline $\mathrm{H}$ & -7.43007000 & -1.76192400 & -0.00706700 & $\mathrm{H}$ & -1.71658800 & 0.28376100 & -1.92047400 \\
\hline $\mathrm{C}$ & -5.91177700 & -3.22257600 & -0.49591000 & $\mathrm{C}$ & 3.83384300 & -1.61608600 & -0.20329600 \\
\hline $\mathrm{H}$ & -5.55825500 & -3.75930700 & -1.38907500 & $\mathrm{C}$ & 2.51810500 & -1.19624900 & -0.39580700 \\
\hline $\mathrm{H}$ & -6.33619800 & -3.98181400 & 0.17457800 & $\mathrm{C}$ & 2.15538800 & 0.14649900 & -0.20867100 \\
\hline $\mathrm{C}$ & -4.71749300 & -2.53043100 & 0.18112400 & $\mathrm{C}$ & 3.15061200 & 1.05938800 & 0.15708600 \\
\hline $\mathrm{H}$ & -5.05427400 & -2.07840200 & 1.12809000 & $\mathrm{C}$ & 4.46871000 & 0.64062100 & 0.35623100 \\
\hline $\mathrm{H}$ & -3.95998300 & -3.27413400 & 0.44519900 & $\mathrm{C}$ & 4.81535400 & -0.69872100 & 0.18073600 \\
\hline $\mathrm{C}$ & -1.31368600 & -3.77166300 & 2.73168400 & $\mathrm{H}$ & 4.09341000 & -2.66078000 & -0.35485800 \\
\hline $\mathrm{C}$ & -1.40036600 & -2.60055900 & 1.97930300 & $\mathrm{H}$ & 1.76933600 & -1.92258800 & -0.70307200 \\
\hline $\mathrm{C}$ & -1.36047300 & -2.64074100 & 0.57832700 & $\mathrm{H}$ & 2.89176200 & 2.10780200 & 0.26240000 \\
\hline $\mathrm{C}$ & -1.21090700 & -3.87986900 & -0.05265800 & $\mathrm{H}$ & 5.22573800 & 1.36680800 & 0.64162900 \\
\hline $\mathrm{C}$ & -1.12647900 & -5.05570100 & 0.69752100 & $\mathrm{H}$ & 5.84059900 & -1.02534900 & 0.33304800 \\
\hline $\mathrm{C}$ & -1.18181900 & -5.00741400 & 2.09139700 & $\mathrm{C}$ & -0.28360500 & -0.18534400 & 0.45860000 \\
\hline $\mathrm{H}$ & -1.34943100 & -3.72131800 & 3.81760900 & $\mathrm{H}$ & -0.00376400 & -0.01302100 & 1.51039200 \\
\hline $\mathrm{H}$ & -1.50121700 & -1.64280800 & 2.48653400 & $\mathrm{H}$ & -0.14901500 & -1.26182400 & 0.28964400 \\
\hline $\mathrm{H}$ & -1.17562300 & -3.89709200 & -1.13684700 & $\mathrm{C}$ & -1.76487200 & 0.18299400 & 0.25841300 \\
\hline $\mathrm{H}$ & -1.01773700 & -6.01224500 & 0.19124900 & $\mathrm{H}$ & -1.85122300 & 1.27576200 & 0.34807500 \\
\hline $\mathrm{H}$ & -1.11831600 & -5.92229900 & 2.67549400 & $\mathrm{O}$ & 0.54782900 & 2.00602400 & -0.22908400 \\
\hline $\mathrm{C}$ & -1.52838700 & -1.37159300 & -0.26427400 & $\mathrm{H}$ & 0.62678700 & 2.17850800 & 0.72407400 \\
\hline $\mathrm{O}$ & -1.24763700 & -1.54182600 & -1.57755400 & $\mathrm{C}$ & 0.71023600 & 0.60085600 & -0.42081100 \\
\hline $\mathrm{C}$ & -2.91582300 & -0.69129200 & -0.04349300 & $\mathrm{H}$ & 0.45709600 & 0.44949600 & -1.47699300 \\
\hline $\mathrm{H}$ & -2.83623200 & 0.30622600 & -0.49059000 & & & & \\
\hline $\mathrm{H}$ & -3.10870700 & -0.53349100 & 1.02674200 & c1 & & & \\
\hline $\mathrm{C}$ & -4.10320900 & -1.42570000 & -0.70402300 & $\mathrm{C}$ & 2.31848000 & -0.17512200 & 0.03423700 \\
\hline \multirow[t]{2}{*}{$\mathrm{H}$} & -3.71597900 & -1.88877900 & -1.62271200 & $\mathrm{C}$ & 1.74546300 & 1.09711800 & 0.00419900 \\
\hline & & & & $\mathrm{C}$ & 0.36014300 & 1.28545700 & -0.05012000 \\
\hline bpro5 & & & & $\mathrm{C}$ & -0.50014800 & 0.16312400 & -0.06316000 \\
\hline $\mathrm{C}$ & -4.12537400 & -0.11488700 & 1.19586600 & $\mathrm{C}$ & 0.06280900 & -1.13348400 & -0.01684400 \\
\hline $\mathrm{H}$ & -4.26677000 & 0.96647500 & 1.34091500 & $\mathrm{C}$ & 1.45567900 & -1.27159100 & 0.02390000 \\
\hline $\mathrm{H}$ & -4.71354000 & -0.61365100 & 1.97705100 & $\mathrm{C}$ & -0.15654600 & 2.71194400 & -0.09096900 \\
\hline $\mathrm{C}$ & -2.63587600 & -0.45475500 & 1.35917200 & $\mathrm{H}$ & -0.72703800 & 2.91940500 & -1.00495400 \\
\hline $\mathrm{H}$ & -2.28178000 & -0.13157100 & 2.34780900 & $\mathrm{H}$ & -0.80981700 & 2.94787500 & 0.75831600 \\
\hline $\mathrm{H}$ & -2.50902200 & -1.54838200 & 1.32787400 & $\mathrm{H}$ & 0.67894000 & 3.41817100 & -0.06206700 \\
\hline $\mathrm{C}$ & -4.64190400 & -0.50843900 & -0.19598700 & $\mathrm{C}$ & 3.81835900 & -0.35862600 & 0.04751700 \\
\hline $\mathrm{H}$ & -5.69300300 & -0.21365000 & -0.31043200 & $\mathrm{H}$ & 4.21995700 & -0.43265500 & -0.97228900 \\
\hline $\mathrm{H}$ & -4.61115000 & -1.60379400 & -0.29662700 & $\mathrm{H}$ & 4.32161300 & 0.48457200 & 0.53322500 \\
\hline
\end{tabular}




\begin{tabular}{|c|c|c|c|c|c|c|c|}
\hline $\mathrm{H}$ & 4.10589000 & -1.27459600 & 0.57530600 & $\mathrm{C}$ & -5.04699800 & -2.65288200 & 0.22648700 \\
\hline $\mathrm{C}$ & -0.76332900 & -2.40543900 & 0.01837600 & $\mathrm{C}$ & -4.26807700 & -1.65381500 & -0.37590400 \\
\hline $\mathrm{H}$ & -1.66983600 & -2.31481100 & -0.57977500 & $\mathrm{C}$ & -2.26275500 & -4.47729700 & -0.07040200 \\
\hline $\mathrm{H}$ & -0.17496700 & -3.24973700 & -0.35651500 & $\mathrm{C}$ & -1.90497800 & -5.66548900 & 0.56716200 \\
\hline $\mathrm{H}$ & -1.05980700 & -2.65749600 & 1.04610700 & $\mathrm{C}$ & -1.27183700 & -5.62766600 & 1.81240400 \\
\hline $\mathrm{C}$ & -2.70205300 & 0.06113000 & 1.23130900 & $\mathrm{C}$ & -0.99745900 & -4.39872400 & 2.41345600 \\
\hline $\mathrm{H}$ & -2.58414200 & -0.99221500 & 1.49814000 & $\mathrm{C}$ & -1.34756700 & -3.20852100 & 1.77114400 \\
\hline $\mathrm{H}$ & -2.28845100 & 0.67036700 & 2.04294800 & $\mathrm{C}$ & -1.98657000 & -3.23304700 & 0.52348800 \\
\hline $\mathrm{H}$ & -3.77716200 & 0.27952500 & 1.16734600 & $\mathrm{H}$ & 0.76094700 & 2.87805300 & -2.45788600 \\
\hline $\mathrm{H}$ & 2.39323100 & 1.97170500 & 0.01569800 & $\mathrm{H}$ & 1.82877000 & 1.82825500 & -1.52155200 \\
\hline $\mathrm{H}$ & 1.87569000 & -2.27575500 & 0.05105800 & $\mathrm{H}$ & -0.76263400 & 0.99199500 & -2.93969100 \\
\hline $\mathrm{O}$ & -2.57085600 & -0.36203000 & -1.18668400 & $\mathrm{H}$ & 0.89642100 & 0.46877500 & -3.30779100 \\
\hline $\mathrm{H}$ & -3.53261200 & -0.23726800 & -1.15117600 & $\mathrm{H}$ & -2.11624300 & -2.87877300 & -2.43784000 \\
\hline $\mathrm{C}$ & -2.00847400 & 0.38990300 & -0.09972900 & $\mathrm{H}$ & -2.43151000 & -1.14978200 & -2.66053500 \\
\hline \multirow[t]{2}{*}{$\mathrm{H}$} & -2.18694300 & 1.45267700 & -0.30019700 & $\mathrm{H}$ & 0.15828400 & -2.41249000 & -1.58903900 \\
\hline & & & & $\mathrm{H}$ & -0.02856000 & -1.67568800 & -3.18371300 \\
\hline 24 & & & & $\mathrm{H}$ & -1.90668700 & 3.14426300 & -2.39090300 \\
\hline $\mathrm{Ru}$ & -1.28408800 & 0.36382600 & 0.18086100 & $\mathrm{H}$ & -3.24220600 & 5.15804900 & -2.87676000 \\
\hline $\mathrm{P}$ & -0.14071900 & 2.36075300 & -0.18112100 & $\mathrm{H}$ & -3.28652400 & 7.05119800 & -1.26072600 \\
\hline $\mathrm{P}$ & -2.42073100 & -1.62629200 & -0.28467500 & $\mathrm{H}$ & -1.95063700 & 6.90992900 & 0.83400600 \\
\hline $\mathrm{N}$ & 0.03804700 & -0.33083400 & -1.52834200 & $\mathrm{H}$ & -0.56266500 & 4.92627900 & 1.29111600 \\
\hline $\mathrm{C}$ & 0.78180300 & 2.00937800 & -1.79162200 & $\mathrm{H}$ & 0.71831800 & 1.60551700 & 2.48489000 \\
\hline $\mathrm{C}$ & 0.21360200 & 0.77425200 & -2.49886500 & $\mathrm{H}$ & 2.50950100 & 2.41197500 & 4.01789700 \\
\hline $\mathrm{C}$ & -1.87817800 & -1.87813600 & -2.05957000 & $\mathrm{H}$ & 4.03775300 & 4.23420700 & 3.29052300 \\
\hline $\mathrm{C}$ & -0.37061400 & -1.62854900 & -2.13999400 & $\mathrm{H}$ & 3.75346100 & 5.26441400 & 1.04463500 \\
\hline $\mathrm{C}$ & -1.88404800 & 3.97945700 & -1.69708100 & $\mathrm{H}$ & 1.94507000 & 4.49750300 & -0.45202900 \\
\hline $\mathrm{C}$ & -2.65393000 & 5.11043100 & -1.96393100 & $\mathrm{H}$ & -4.32876400 & 0.20201600 & -1.47170500 \\
\hline $\mathrm{C}$ & -2.68015700 & 6.17276600 & -1.05655400 & $\mathrm{H}$ & -6.79556400 & 0.23820500 & -1.67213300 \\
\hline $\mathrm{C}$ & -1.93036100 & 6.09383700 & 0.11648600 & $\mathrm{H}$ & -8.16072400 & -1.55608500 & -0.61578500 \\
\hline $\mathrm{C}$ & -1.14635200 & 4.96694700 & 0.37710100 & $\mathrm{H}$ & -7.02815900 & -3.39519000 & 0.61928800 \\
\hline $\mathrm{C}$ & -1.10809000 & 3.89797800 & -0.52970500 & $\mathrm{H}$ & -4.57182700 & -3.46304400 & 0.76862900 \\
\hline $\mathrm{C}$ & 1.37845700 & 2.41421500 & 2.18374000 & $\mathrm{H}$ & -2.77242800 & -4.52516300 & -1.02922400 \\
\hline $\mathrm{C}$ & 2.39191600 & 2.86347100 & 3.03627100 & $\mathrm{H}$ & -2.12146800 & -6.61952100 & 0.09336300 \\
\hline $\mathrm{C}$ & 3.24911000 & 3.88619300 & 2.62850800 & $\mathrm{H}$ & -0.99292900 & -6.55340300 & 2.30900000 \\
\hline $\mathrm{C}$ & 3.08961300 & 4.46597700 & 1.36593700 & $\mathrm{H}$ & -0.50337900 & -4.36302900 & 3.38081800 \\
\hline $\mathrm{C}$ & 2.07320000 & 4.02484100 & 0.51860400 & $\mathrm{H}$ & -1.11049400 & -2.24826100 & 2.21986500 \\
\hline $\mathrm{C}$ & 1.20814000 & 2.99069000 & 0.91784900 & $\mathrm{C}$ & -2.36131400 & 0.94671000 & 1.57242700 \\
\hline $\mathrm{C}$ & -4.91915400 & -0.60589200 & -1.04938100 & $\mathrm{O}$ & -3.03211200 & 1.30948500 & 2.45198600 \\
\hline $\mathrm{C}$ & -6.30956900 & -0.57864000 & -1.14479400 & $\mathrm{H}$ & -0.16773100 & -0.30139800 & 1.31275200 \\
\hline $\mathrm{C}$ & -7.07621100 & -1.58377200 & -0.54970500 & $\mathrm{H}$ & -2.31387000 & 0.98150300 & -1.00496900 \\
\hline $\mathrm{C}$ & -6.44121000 & -2.61516000 & 0.14123500 & $\mathrm{H}$ & 0.94245900 & -0.50871500 & -1.07587800 \\
\hline
\end{tabular}




\begin{tabular}{|c|c|c|c|c|c|c|c|}
\hline C & 6.62131200 & -1.85178300 & -1.60772500 & $\mathrm{C}$ & -0.20754600 & 6.11824400 & -1.85474800 \\
\hline C & 6.42345600 & -0.57752000 & -1.07303600 & C & -0.14836900 & 5.96066800 & -0.46847400 \\
\hline C & 5.33147600 & -0.27893500 & -0.25060500 & $\mathrm{C}$ & 0.19554300 & 4.72665600 & 0.08388500 \\
\hline C & 4.39387000 & -1.29021300 & 0.06617400 & $\mathrm{C}$ & 0.48647400 & 3.62931300 & -0.74218400 \\
\hline C & 4.59260200 & -2.59256400 & -0.44809700 & $\mathrm{C}$ & 1.86936300 & 1.88868600 & 2.63603600 \\
\hline C & 5.69375400 & -2.84019000 & -1.27779100 & $\mathrm{C}$ & 2.78669000 & 2.17905700 & 3.64966800 \\
\hline C & 5.21274900 & 1.13838700 & 0.27812800 & $\mathrm{C}$ & 3.88238600 & 3.00112600 & 3.38700300 \\
\hline $\mathrm{H}$ & 4.29476100 & 1.63788600 & -0.05526000 & $\mathrm{C}$ & 4.06013000 & 3.53790100 & 2.10820100 \\
\hline $\mathrm{H}$ & 5.21121800 & 1.17970500 & 1.37383700 & $\mathrm{C}$ & 3.14413400 & 3.25189600 & 1.09683000 \\
\hline $\mathrm{H}$ & 6.05633800 & 1.74350100 & -0.06884800 & $\mathrm{C}$ & 2.04001600 & 2.41913000 & 1.35024900 \\
\hline $\mathrm{C}$ & 7.78336000 & -2.14245300 & -2.52922800 & $\mathrm{C}$ & -4.60340700 & 1.05054900 & -1.10504600 \\
\hline $\mathrm{H}$ & 7.52320100 & -1.94038900 & -3.57736700 & $\mathrm{C}$ & -5.86166300 & 1.54036300 & -1.45755900 \\
\hline $\mathrm{H}$ & 8.65281700 & -1.52128600 & -2.28699200 & $\mathrm{C}$ & -6.97906900 & 0.70484600 & -1.41104300 \\
\hline $\mathrm{H}$ & 8.09020500 & -3.19236600 & -2.46891600 & $\mathrm{C}$ & -6.83271800 & -0.62473100 & -1.01127100 \\
\hline C & 3.69117700 & -3.76907700 & -0.12266100 & $\mathrm{C}$ & -5.57503300 & -1.11899400 & -0.66257400 \\
\hline $\mathrm{H}$ & 2.65775800 & -3.46229500 & 0.03580700 & $\mathrm{C}$ & -4.44452100 & -0.28758800 & -0.71030500 \\
\hline $\mathrm{H}$ & 3.72166100 & -4.50634800 & -0.93268800 & $\mathrm{C}$ & -3.22317900 & -3.70141800 & 0.17214600 \\
\hline $\mathrm{H}$ & 4.03216000 & -4.28421600 & 0.78615700 & $\mathrm{C}$ & -3.45684700 & -4.79734700 & 1.00601900 \\
\hline C & 3.32507400 & -1.56089100 & 2.37524500 & $\mathrm{C}$ & -3.54035300 & -4.62177700 & 2.38768600 \\
\hline $\mathrm{H}$ & 3.33657800 & -2.65331700 & 2.33892300 & $\mathrm{C}$ & -3.38427300 & -3.34627300 & 2.93501300 \\
\hline $\mathrm{H}$ & 4.24393300 & -1.22264900 & 2.86868900 & C & -3.14016400 & -2.25376400 & 2.10388800 \\
\hline $\mathrm{H}$ & 2.47307900 & -1.24753500 & 2.99090500 & $\mathrm{C}$ & -3.06271500 & -2.41562900 & 0.71100600 \\
\hline $\mathrm{H}$ & 7.13646700 & 0.21185500 & -1.30438100 & $\mathrm{H}$ & 2.40092600 & 1.72537700 & -1.91297100 \\
\hline $\mathrm{H}$ & 5.82911200 & -3.84610800 & -1.67239900 & $\mathrm{H}$ & 2.61761200 & 0.49541400 & -0.66527100 \\
\hline $\mathrm{O}$ & 1.99026700 & -1.35079300 & 0.33118500 & $\mathrm{H}$ & 0.35805800 & 0.58178400 & -2.73426600 \\
\hline $\mathrm{H}$ & 1.22438000 & -1.01019500 & 0.86135800 & $\mathrm{H}$ & 1.64812300 & -0.62360600 & -2.56740700 \\
\hline C & 3.21236700 & -0.94847000 & 0.97081400 & $\mathrm{H}$ & -2.61857400 & -2.36888000 & -2.34569800 \\
\hline \multirow[t]{2}{*}{$\mathrm{H}$} & 3.18073100 & 0.13816200 & 1.09859600 & $\mathrm{H}$ & -2.20944600 & -0.67187300 & -2.63078500 \\
\hline & & & & $\mathrm{H}$ & -0.48654500 & -2.70819800 & -1.10176900 \\
\hline \multirow{2}{*}{\multicolumn{4}{|c|}{$\begin{array}{l}\text { TS32 } \\
\text { imaginary frequency }=-991.70 \mathrm{~cm}^{-1}\end{array}$}} & $\mathrm{H}$ & -0.12291200 & -2.05738200 & -2.69739900 \\
\hline & & & & $\mathrm{H}$ & 0.62034200 & 2.96129900 & -2.79672600 \\
\hline $\mathrm{R}$ & -1.02086800 & 0.56519800 & 0.28981600 & $\mathrm{H}$ & 0.03147500 & 5.14493700 & -3.76494600 \\
\hline $\mathrm{P}$ & 0.84389500 & 1.98632000 & 0.01517300 & $\mathrm{H}$ & -0.47353900 & 7.08010000 & -2.28466700 \\
\hline $\mathrm{P}$ & -2.75511200 & -0.91429900 & -0.31100500 & $\mathrm{H}$ & -0.36852900 & 6.79942900 & 0.18646200 \\
\hline $\mathrm{N}$ & 0.15916200 & -0.71996000 & -1.06382700 & $\mathrm{H}$ & 0.24410400 & 4.62111000 & 1.16409600 \\
\hline C & 1.86674800 & 1.04970800 & -1.23630000 & $\mathrm{H}$ & 1.01946500 & 1.24566800 & 2.84140200 \\
\hline C & 0.98838400 & 0.05399700 & -2.00304400 & $\mathrm{H}$ & 2.64230300 & 1.76114500 & 4.64215900 \\
\hline C & -2.07456400 & -1.50902000 & -1.93660300 & $\mathrm{H}$ & 4.59631200 & 3.22624600 & 4.17476400 \\
\hline C & -0.58528600 & -1.81526300 & -1.73010600 & $\mathrm{H}$ & 4.91134500 & 4.18065000 & 1.89998300 \\
\hline C & 0.41675800 & 3.79626400 & -2.13303900 & $\mathrm{H}$ & 3.28300900 & 3.68461000 & 0.10972000 \\
\hline C & 0.07549200 & 5.03256100 & -2.68479500 & $\mathrm{H}$ & -3.73936200 & 1.70710600 & -1.12849800 \\
\hline
\end{tabular}




\begin{tabular}{|c|c|c|c|c|c|c|c|}
\hline $\mathrm{H}$ & -5.96821100 & 2.57907200 & -1.75877500 & $\mathrm{H}$ & 2.85280700 & -0.95501900 & 1.50404400 \\
\hline $\mathrm{H}$ & -7.95984300 & 1.08917900 & -1.67842700 & & & & \\
\hline $\mathrm{H}$ & -7.69878400 & -1.27983700 & -0.96639000 & 25 & & & \\
\hline $\mathrm{H}$ & -5.47741900 & -2.15267200 & -0.34606000 & $\mathrm{Ru}$ & 0.94819900 & 0.63601100 & 0.31085100 \\
\hline $\mathrm{H}$ & -3.16536400 & -3.86041300 & -0.90028200 & $\mathrm{P}$ & 2.48524500 & -1.08371700 & -0.21859200 \\
\hline $\mathrm{H}$ & -3.57027000 & -5.78779600 & 0.57346100 & $\mathrm{P}$ & -0.65858900 & 2.31771700 & -0.09934300 \\
\hline $\mathrm{H}$ & -3.71958400 & -5.47541500 & 3.03567400 & $\mathrm{~N}$ & -0.24447600 & -0.48198700 & -1.12310400 \\
\hline $\mathrm{H}$ & -3.44118200 & -3.20297500 & 4.01057600 & $\mathrm{C}$ & 1.65335100 & -1.92212100 & -1.66730600 \\
\hline $\mathrm{H}$ & -2.99706300 & -1.26805800 & 2.53817400 & $\mathrm{C}$ & 0.56185700 & -0.99461600 & -2.22738500 \\
\hline $\mathrm{C}$ & -2.01905800 & 1.70307800 & 1.40328400 & $\mathrm{C}$ & -1.38783800 & 1.69187800 & -1.68703900 \\
\hline $\mathrm{O}$ & -2.64843600 & 2.42158200 & 2.06414400 & $\mathrm{C}$ & -1.48943400 & 0.15834800 & -1.55432300 \\
\hline $\mathrm{H}$ & -0.48140000 & -0.38969600 & 1.89665400 & $\mathrm{C}$ & 4.22480800 & 0.11093000 & -2.08801500 \\
\hline $\mathrm{H}$ & -1.56042500 & 1.38945800 & -0.96586300 & $\mathrm{C}$ & 5.45354200 & 0.56432100 & -2.56601500 \\
\hline $\mathrm{H}$ & 0.85759900 & -1.31153000 & -0.21289600 & $\mathrm{C}$ & 6.61227900 & 0.38738100 & -1.80546600 \\
\hline $\mathrm{C}$ & 5.36116000 & -2.76706600 & -2.17195100 & $\mathrm{C}$ & 6.53213200 & -0.24753200 & -0.56623400 \\
\hline $\mathrm{C}$ & 5.60712600 & -1.75597600 & -1.24043600 & $\mathrm{C}$ & 5.30320900 & -0.71154600 & -0.09049600 \\
\hline $\mathrm{C}$ & 4.74193100 & -1.50678400 & -0.16864400 & $\mathrm{C}$ & 4.13505700 & -0.54068700 & -0.84689300 \\
\hline $\mathrm{C}$ & 3.56827000 & -2.28550700 & -0.00800300 & $\mathrm{C}$ & 2.71149400 & -2.28500800 & 2.31545700 \\
\hline $\mathrm{C}$ & 3.34206600 & -3.35172400 & -0.90989400 & $\mathrm{C}$ & 3.03868000 & -3.30799500 & 3.20887700 \\
\hline $\mathrm{C}$ & 4.22943200 & -3.55833500 & -1.97516200 & $\mathrm{C}$ & 3.57109000 & -4.50664300 & 2.73399500 \\
\hline $\mathrm{C}$ & 5.13509800 & -0.41681000 & 0.81287200 & $\mathrm{C}$ & 3.78306800 & -4.67924400 & 1.36321000 \\
\hline $\mathrm{H}$ & 4.42641100 & 0.41985700 & 0.83554300 & $\mathrm{C}$ & 3.46400800 & -3.65540300 & 0.47229400 \\
\hline $\mathrm{H}$ & 5.20494300 & -0.79402900 & 1.83995100 & $\mathrm{C}$ & 2.91925100 & -2.44757200 & 0.93965900 \\
\hline $\mathrm{H}$ & 6.11500200 & -0.00576000 & 0.54771200 & $\mathrm{C}$ & 1.18373300 & 4.37071100 & -0.69284000 \\
\hline $\mathrm{C}$ & 6.28207500 & -2.98916900 & -3.34986700 & $\mathrm{C}$ & 1.56029100 & 5.67874900 & -1.00593600 \\
\hline $\mathrm{H}$ & 5.98728800 & -2.37702100 & -4.21376000 & $\mathrm{C}$ & 0.59984600 & 6.68862000 & -1.07326800 \\
\hline $\mathrm{H}$ & 7.31717200 & -2.72392900 & -3.10616500 & $\mathrm{C}$ & -0.74208500 & 6.38820200 & -0.82549900 \\
\hline $\mathrm{H}$ & 6.26996400 & -4.03495300 & -3.67682800 & $\mathrm{C}$ & -1.12064000 & 5.08334400 & -0.51154700 \\
\hline $\mathrm{C}$ & 2.20858700 & -4.34685400 & -0.74402000 & $\mathrm{C}$ & -0.16102200 & 4.05894200 & -0.44566000 \\
\hline $\mathrm{H}$ & 1.39678300 & -3.92208700 & -0.15419400 & $\mathrm{C}$ & -3.38345200 & 2.18750600 & 0.79084800 \\
\hline $\mathrm{H}$ & 1.82618800 & -4.66777200 & -1.72115000 & $\mathrm{C}$ & -4.38399100 & 2.32067000 & 1.75860200 \\
\hline $\mathrm{H}$ & 2.56882800 & -5.25162100 & -0.23381600 & $\mathrm{C}$ & -4.07127000 & 2.79052200 & 3.03364800 \\
\hline $\mathrm{C}$ & 2.82362600 & -2.91279200 & 2.34061300 & $\mathrm{C}$ & -2.74934500 & 3.12429500 & 3.34284900 \\
\hline $\mathrm{H}$ & 2.60412700 & -3.94904700 & 2.06350600 & $\mathrm{C}$ & -1.74973000 & 2.98309200 & 2.38259000 \\
\hline $\mathrm{H}$ & 3.85916200 & -2.86591900 & 2.70201000 & $\mathrm{C}$ & -2.05424000 & 2.51838100 & 1.09047000 \\
\hline $\mathrm{H}$ & 2.15333300 & -2.63401600 & 3.16209600 & $\mathrm{H}$ & 2.38126000 & -2.21685300 & -2.43060100 \\
\hline $\mathrm{H}$ & 6.50128800 & -1.14311300 & -1.34617600 & $\mathrm{H}$ & 1.18286200 & -2.82140200 & -1.25794200 \\
\hline $\mathrm{H}$ & 4.03023900 & -4.37913700 & -2.66372900 & $\mathrm{H}$ & 1.02972200 & -0.20125400 & -2.84115600 \\
\hline $\mathrm{O}$ & 1.24047800 & -2.00292700 & 0.77940800 & $\mathrm{H}$ & -0.06497400 & -1.58593400 & -2.91899800 \\
\hline $\mathrm{H}$ & 0.06307600 & -0.85810400 & 1.54091500 & $\mathrm{H}$ & -2.34454800 & 2.16912200 & -1.93505000 \\
\hline $\mathrm{C}$ & 2.59179500 & -1.96641400 & 1.14566400 & $\mathrm{H}$ & -0.66696000 & 1.96419200 & -2.46656900 \\
\hline
\end{tabular}




\begin{tabular}{|c|c|c|c|c|c|c|c|}
\hline $\mathrm{H}$ & -2.27662600 & -0.07565900 & -0.82387500 & $\mathrm{C}$ & -3.46847200 & -2.10346500 & 1.90930400 \\
\hline $\mathrm{H}$ & -1.83248700 & -0.26188600 & -2.51272800 & $\mathrm{H}$ & -2.38010700 & -2.10061600 & 1.96178000 \\
\hline $\mathrm{H}$ & 3.33004400 & 0.27415000 & -2.68201200 & $\mathrm{H}$ & -3.85328100 & -1.17655000 & 2.34969100 \\
\hline $\mathrm{H}$ & 5.50516600 & 1.05984300 & -3.53207500 & $\mathrm{H}$ & -3.83522200 & -2.93076400 & 2.53316500 \\
\hline $\mathrm{H}$ & 7.56923100 & 0.74445800 & -2.17652700 & $\mathrm{C}$ & -1.93217900 & -4.83759100 & 0.58959600 \\
\hline $\mathrm{H}$ & 7.42679800 & -0.38751700 & 0.03474700 & $\mathrm{H}$ & -2.35532500 & -4.63720000 & 1.57832900 \\
\hline $\mathrm{H}$ & 5.25924200 & -1.21224800 & 0.87157400 & $\mathrm{H}$ & -2.56083300 & -5.58560300 & 0.09264900 \\
\hline $\mathrm{H}$ & 2.28782800 & -1.35655400 & 2.68726800 & $\mathrm{H}$ & -0.92829900 & -5.25393600 & 0.72721300 \\
\hline $\mathrm{H}$ & 2.87024100 & -3.16835800 & 4.27321800 & $\mathrm{H}$ & -5.48859100 & -2.64481300 & -3.06476700 \\
\hline $\mathrm{H}$ & 3.81971900 & -5.30553600 & 3.42752300 & $\mathrm{H}$ & -5.74548800 & -1.16595200 & 0.94398000 \\
\hline $\mathrm{H}$ & 4.19748400 & -5.61150300 & 0.98859400 & $\mathrm{O}$ & -0.93846800 & -2.67708300 & 0.40611700 \\
\hline $\mathrm{H}$ & 3.64201300 & -3.79737200 & -0.59036500 & $\mathrm{H}$ & 0.17231700 & -0.60537100 & 1.63256100 \\
\hline $\mathrm{H}$ & 1.93243500 & 3.58737400 & -0.63635900 & $\mathrm{C}$ & -1.84489900 & -3.55460000 & -0.24577900 \\
\hline $\mathrm{H}$ & 2.60672500 & 5.90669600 & -1.19021000 & $\mathrm{H}$ & -1.41609000 & -3.83824500 & -1.21684700 \\
\hline $\mathrm{H}$ & 0.89443800 & 7.70697800 & -1.31280100 & & & & \\
\hline $\mathrm{H}$ & -1.49446100 & 7.17111600 & -0.87281800 & d1 & & & \\
\hline $\mathrm{H}$ & -2.16486800 & 4.86284500 & -0.30890200 & $\mathrm{C}$ & -0.19135900 & 1.16840900 & 1.25685200 \\
\hline $\mathrm{H}$ & -3.65213200 & 1.81481300 & -0.19178100 & $\mathrm{H}$ & -1.11394700 & 1.75965400 & 1.27269100 \\
\hline $\mathrm{H}$ & -5.40783400 & 2.05495600 & 1.50938300 & $\mathrm{H}$ & 0.37397600 & 1.41908400 & 2.16530000 \\
\hline $\mathrm{H}$ & -4.85013600 & 2.89372100 & 3.78431500 & $\mathrm{C}$ & 0.63921100 & 1.50535800 & -0.00000100 \\
\hline $\mathrm{H}$ & -2.49603400 & 3.48980700 & 4.33439800 & $\mathrm{H}$ & 0.88928800 & 2.57464100 & -0.00000100 \\
\hline $\mathrm{H}$ & -0.72354000 & 3.23666400 & 2.63756900 & $\mathrm{C}$ & -0.53938300 & -0.33490000 & 1.25993400 \\
\hline $\mathrm{C}$ & 2.04114700 & 1.59074300 & 1.51831600 & $\mathrm{H}$ & -1.14235900 & -0.57601100 & 2.14527700 \\
\hline $\mathrm{O}$ & 2.72986500 & 2.20597900 & 2.22498500 & $\mathrm{C}$ & -1.36190100 & -0.67498700 & 0.00000000 \\
\hline $\mathrm{H}$ & -0.28340800 & 0.02617900 & 1.71003400 & $\mathrm{H}$ & -1.58492400 & -1.75319900 & 0.00000100 \\
\hline $\mathrm{H}$ & 1.69886900 & 1.35719200 & -0.89595500 & $\mathrm{C}$ & -0.19135700 & 1.16840700 & -1.25685400 \\
\hline $\mathrm{H}$ & -0.74730800 & -1.88045300 & -0.18573900 & $\mathrm{H}$ & 0.37398000 & 1.41907800 & -2.16530300 \\
\hline $\mathrm{C}$ & -5.77802200 & -1.81752200 & -1.10143900 & $\mathrm{H}$ & -1.11394500 & 1.75965200 & -1.27269700 \\
\hline $\mathrm{C}$ & -5.06140600 & -2.52479400 & -2.07029100 & $\mathrm{C}$ & -0.53938200 & -0.33490200 & -1.25993400 \\
\hline $\mathrm{C}$ & -3.80683600 & -3.08231500 & -1.80393500 & $\mathrm{H}$ & -1.14235800 & -0.57601200 & -2.14527600 \\
\hline $\mathrm{C}$ & -3.22874900 & -2.94250300 & -0.51797200 & $\mathrm{C}$ & 0.75951900 & -1.16978300 & -1.25639600 \\
\hline $\mathrm{C}$ & -3.95424900 & -2.25208400 & 0.47982300 & $\mathrm{H}$ & 0.52391900 & -2.24369300 & -1.27642600 \\
\hline $\mathrm{C}$ & -5.20369700 & -1.69876000 & 0.16349400 & $\mathrm{H}$ & 1.34010800 & -0.95611900 & -2.16431000 \\
\hline $\mathrm{C}$ & -3.11702500 & -3.83428900 & -2.92795300 & $\mathrm{C}$ & 1.93735100 & 0.66996300 & -0.00000100 \\
\hline $\mathrm{H}$ & -2.16635300 & -3.36886700 & -3.21853800 & $\mathrm{H}$ & 2.54460500 & 0.91493200 & 0.88282800 \\
\hline $\mathrm{H}$ & -2.89460100 & -4.87455000 & -2.66121400 & $\mathrm{H}$ & 2.54460400 & 0.91493100 & -0.88283100 \\
\hline $\mathrm{H}$ & -3.75479500 & -3.85517900 & -3.81752500 & $\mathrm{C}$ & 0.75952000 & -1.16977900 & 1.25639900 \\
\hline $\mathrm{C}$ & -7.11779900 & -1.19502700 & -1.42194500 & $\mathrm{H}$ & 1.34010800 & -0.95611100 & 2.16431200 \\
\hline $\mathrm{H}$ & -7.00463000 & -0.27264000 & -2.00808300 & $\mathrm{H}$ & 0.52392100 & -2.24369000 & 1.27643300 \\
\hline $\mathrm{H}$ & -7.74513300 & -1.87301000 & -2.01265500 & $\mathrm{C}$ & 1.59016600 & -0.83433300 & 0.00000100 \\
\hline $\mathrm{H}$ & -7.66756500 & -0.93794100 & -0.51015600 & $\mathrm{H}$ & 2.51404300 & -1.42794600 & 0.00000100 \\
\hline
\end{tabular}




\begin{tabular}{|c|c|c|c|c|c|c|c|}
\hline $\mathrm{O}$ & -2.58985600 & 0.07059900 & -0.00000100 & $\mathrm{H}$ & -1.70880700 & 2.30936500 & 2.47652500 \\
\hline \multirow[t]{2}{*}{$\mathrm{H}$} & -3.32647100 & -0.55470000 & 0.00000500 & $\mathrm{H}$ & 1.25397600 & 1.54944600 & 2.24290100 \\
\hline & & & & $\mathrm{H}$ & 0.20826000 & 1.18281900 & 3.61674700 \\
\hline 26 & & & & $\mathrm{H}$ & -3.05091600 & -4.43704500 & 1.37447000 \\
\hline $\mathrm{Ru}$ & -1.15516200 & 0.21473800 & -0.15231200 & $\mathrm{H}$ & -5.41865700 & -5.04384900 & 1.69643600 \\
\hline $\mathrm{P}$ & -1.70341800 & -2.02608700 & 0.26130900 & $\mathrm{H}$ & -7.21861500 & -3.49719400 & 0.94781400 \\
\hline $\mathrm{P}$ & -0.80211200 & 2.48774800 & 0.23943300 & $\mathrm{H}$ & -6.61944500 & -1.32635400 & -0.10869400 \\
\hline $\mathrm{N}$ & -0.11958800 & 0.01769400 & 1.85926500 & $\mathrm{H}$ & -4.23324400 & -0.69218400 & -0.37701100 \\
\hline $\mathrm{C}$ & -0.97917500 & -2.28358400 & 1.98025500 & $\mathrm{H}$ & 1.03981800 & -3.00468300 & -0.17186900 \\
\hline $\mathrm{C}$ & -0.86189100 & -0.94274100 & 2.71061000 & $\mathrm{H}$ & 2.00266200 & -4.82134300 & -1.51122400 \\
\hline $\mathrm{C}$ & -0.69008800 & 2.46428500 & 2.10909500 & $\mathrm{H}$ & 0.53783000 & -6.27499800 & -2.90498700 \\
\hline $\mathrm{C}$ & 0.22315100 & 1.30869100 & 2.52523400 & $\mathrm{H}$ & -1.92107600 & -5.88767800 & -2.91634600 \\
\hline $\mathrm{C}$ & -3.82616300 & -3.74088000 & 1.06422700 & $\mathrm{H}$ & -2.90365600 & -4.10661800 & -1.52989700 \\
\hline $\mathrm{C}$ & -5.16458400 & -4.09208300 & 1.23701400 & $\mathrm{H}$ & -3.63720900 & 2.51298800 & 0.85466900 \\
\hline $\mathrm{C}$ & -6.17511300 & -3.22323800 & 0.81552900 & $\mathrm{H}$ & -5.47593200 & 4.12087300 & 0.45901900 \\
\hline $\mathrm{C}$ & -5.83887000 & -2.00561300 & 0.22374200 & $\mathrm{H}$ & -5.04690700 & 6.22277300 & -0.80449000 \\
\hline $\mathrm{C}$ & -4.49746800 & -1.64914300 & 0.05987000 & $\mathrm{H}$ & -2.75409500 & 6.70768100 & -1.64003200 \\
\hline $\mathrm{C}$ & -3.47595500 & -2.51168500 & 0.47801800 & $\mathrm{H}$ & -0.91070700 & 5.13809300 & -1.19225400 \\
\hline $\mathrm{C}$ & 0.37495900 & -3.65095500 & -0.73767500 & $\mathrm{H}$ & 0.63899700 & 4.96971200 & 1.17360900 \\
\hline $\mathrm{C}$ & 0.92606800 & -4.67056800 & -1.51295700 & $\mathrm{H}$ & 2.74051800 & 6.03858500 & 0.45597100 \\
\hline $\mathrm{C}$ & 0.10513800 & -5.48488900 & -2.29693200 & $\mathrm{H}$ & 4.05134200 & 5.08168000 & -1.42893200 \\
\hline $\mathrm{C}$ & -1.27244000 & -5.26878400 & -2.30174300 & $\mathrm{H}$ & 3.24489100 & 3.02711700 & -2.57694800 \\
\hline $\mathrm{C}$ & -1.82921700 & -4.25662700 & -1.51596700 & $\mathrm{H}$ & 1.15653800 & 1.91213800 & -1.80729000 \\
\hline $\mathrm{C}$ & -1.01470100 & -3.44200100 & -0.71496600 & $\mathrm{C}$ & -1.95258600 & 0.35758500 & -1.81820500 \\
\hline $\mathrm{C}$ & -3.43681900 & 3.44966500 & 0.34260900 & $\mathrm{O}$ & -2.44509200 & 0.44620200 & -2.86982800 \\
\hline $\mathrm{C}$ & -4.47475800 & 4.34841900 & 0.10201700 & $\mathrm{H}$ & 0.34710200 & -0.12812700 & -0.92302600 \\
\hline $\mathrm{C}$ & -4.23492500 & 5.52738000 & -0.60869300 & $\mathrm{H}$ & -2.57162200 & 0.54077100 & 0.70319200 \\
\hline $\mathrm{C}$ & -2.94956300 & 5.79968000 & -1.07546000 & $\mathrm{H}$ & 0.77225200 & -0.42250000 & 1.60630300 \\
\hline $\mathrm{C}$ & -1.90393500 & 4.90782100 & -0.82243600 & $\mathrm{C}$ & 4.62542000 & -2.12140400 & -0.98049100 \\
\hline $\mathrm{C}$ & -2.13271300 & 3.72577500 & -0.10295900 & $\mathrm{H}$ & 3.72571000 & -2.74608200 & -0.93750000 \\
\hline $\mathrm{C}$ & 1.21160400 & 4.52503400 & 0.36375500 & $\mathrm{H}$ & 5.13541600 & -2.35241600 & -1.92602200 \\
\hline $\mathrm{C}$ & 2.39519600 & 5.13911700 & -0.04725100 & $\mathrm{C}$ & 5.55000300 & -2.43269600 & 0.21530800 \\
\hline $\mathrm{C}$ & 3.13117500 & 4.60127400 & -1.10668100 & $\mathrm{H}$ & 5.83317300 & -3.49374000 & 0.19681300 \\
\hline $\mathrm{C}$ & 2.67941100 & 3.44765300 & -1.74953400 & $\mathrm{C}$ & 4.22957800 & -0.63103700 & -0.95478300 \\
\hline $\mathrm{C}$ & 1.49981600 & 2.82578700 & -1.33019200 & $\mathrm{H}$ & 3.56242100 & -0.40662500 & -1.79819700 \\
\hline $\mathrm{C}$ & 0.75082700 & 3.35802900 & -0.27076900 & $\mathrm{C}$ & 3.49337200 & -0.29845900 & 0.36258100 \\
\hline $\mathrm{H}$ & -1.58549500 & -2.98533000 & 2.56133100 & $\mathrm{H}$ & 3.26379800 & 0.77914400 & 0.37333100 \\
\hline $\mathrm{H}$ & 0.01596900 & -2.72622000 & 1.86167500 & $\mathrm{C}$ & 4.79945700 & -2.12073100 & 1.52776400 \\
\hline $\mathrm{H}$ & -1.85075400 & -0.52402200 & 2.91782500 & $\mathrm{H}$ & 5.43636800 & -2.35154900 & 2.39326400 \\
\hline $\mathrm{H}$ & -0.33875800 & -1.08833600 & 3.66932300 & $\mathrm{H}$ & 3.90209500 & -2.74471800 & 1.60890700 \\
\hline $\mathrm{H}$ & -0.31916200 & 3.40783900 & 2.52570900 & $\mathrm{C}$ & 4.40606500 & -0.62851100 & 1.55763300 \\
\hline
\end{tabular}




\begin{tabular}{|c|c|c|c|c|c|c|c|}
\hline $\mathrm{H}$ & 3.86287600 & -0.40450800 & 2.48591400 & $\mathrm{C}$ & -3.42278000 & -4.32784900 & 0.08744900 \\
\hline $\mathrm{C}$ & 5.67681000 & 0.24341100 & 1.46651500 & $\mathrm{C}$ & -3.21006500 & -2.96552300 & -0.17515200 \\
\hline $\mathrm{H}$ & 5.41159200 & 1.30918800 & 1.50853000 & $\mathrm{C}$ & 0.14903300 & -4.55661500 & -0.12967900 \\
\hline $\mathrm{H}$ & 6.32647500 & 0.04243600 & 2.32946400 & $\mathrm{C}$ & 0.95844800 & -5.47802700 & 0.53868800 \\
\hline $\mathrm{C}$ & 6.81852800 & -1.55755800 & 0.12555900 & $\mathrm{C}$ & 1.17951900 & -5.35154500 & 1.91081700 \\
\hline $\mathrm{H}$ & 7.36881000 & -1.78478500 & -0.79834700 & $\mathrm{C}$ & 0.59112800 & -4.29764000 & 2.61345800 \\
\hline $\mathrm{H}$ & 7.49382300 & -1.78368500 & 0.96293300 & $\mathrm{C}$ & -0.20778300 & -3.37091600 & 1.94424500 \\
\hline $\mathrm{C}$ & 5.49858400 & 0.24487500 & -1.04174500 & $\mathrm{C}$ & -0.44361400 & -3.49011300 & 0.56470400 \\
\hline $\mathrm{H}$ & 6.02106100 & 0.04977200 & -1.98830700 & $\mathrm{H}$ & 0.06640300 & 3.21316500 & -2.34349000 \\
\hline $\mathrm{H}$ & 5.22505400 & 1.30947700 & -1.04152500 & $\mathrm{H}$ & 1.29361000 & 2.36017800 & -1.37450700 \\
\hline $\mathrm{C}$ & 6.42581900 & -0.06477100 & 0.15262200 & $\mathrm{H}$ & -1.00053700 & 1.02658700 & -2.91289300 \\
\hline $\mathrm{H}$ & 7.32884200 & 0.55710500 & 0.08955800 & $\mathrm{H}$ & 0.73377300 & 0.95639900 & -3.28134200 \\
\hline $\mathrm{O}$ & 2.27278000 & -1.03361100 & 0.50260600 & $\mathrm{H}$ & -0.97804500 & -3.23866500 & -2.46582400 \\
\hline \multirow[t]{2}{*}{$\mathrm{H}$} & 1.62295500 & -0.71153000 & -0.17891600 & $\mathrm{H}$ & -1.90703100 & -1.73567700 & -2.55134900 \\
\hline & & & & $\mathrm{H}$ & 1.05150400 & -1.98943500 & -1.77001800 \\
\hline \multirow{2}{*}{\multicolumn{4}{|c|}{$\begin{array}{l}\text { TS33 } \\
\text { imaginary frequency }=-843.36 \mathrm{~cm}^{-1}\end{array}$}} & $\mathrm{H}$ & 0.44669100 & -1.29496400 & -3.27085700 \\
\hline & & & & $\mathrm{H}$ & -1.07036000 & 5.03196100 & -1.46164600 \\
\hline $\mathrm{Ru}$ & -1.16179900 & 0.06236400 & 0.21596800 & $\mathrm{H}$ & -3.00462600 & 6.41579700 & -2.10619300 \\
\hline $\mathrm{P}$ & -0.81231800 & 2.38117100 & -0.17172800 & $\mathrm{H}$ & -5.31795900 & 5.63357300 & -1.62802200 \\
\hline $\mathrm{P}$ & -1.52007700 & -2.22678900 & -0.23727400 & $\mathrm{H}$ & -5.67135200 & 3.44385000 & -0.50237400 \\
\hline $\mathrm{N}$ & 0.17550900 & -0.10005500 & -1.52927400 & $\mathrm{H}$ & -3.73010600 & 2.03882500 & 0.11561800 \\
\hline $\mathrm{C}$ & 0.25167300 & 2.34251200 & -1.70710200 & $\mathrm{H}$ & 1.90152000 & 2.19413000 & 0.86971400 \\
\hline $\mathrm{C}$ & 0.00160100 & 1.03155300 & -2.46134200 & $\mathrm{H}$ & 3.17352800 & 3.49767000 & 2.53660700 \\
\hline $\mathrm{C}$ & -1.07189800 & -2.23095100 & -2.04372500 & $\mathrm{H}$ & 2.11830700 & 5.42556600 & 3.70689200 \\
\hline $\mathrm{C}$ & 0.21978600 & -1.41768700 & -2.20249700 & $\mathrm{H}$ & -0.23195000 & 6.04921900 & 3.17980800 \\
\hline $\mathrm{C}$ & -2.07489000 & 4.66834000 & -1.26262000 & $\mathrm{H}$ & -1.50264500 & 4.78278100 & 1.48962900 \\
\hline $\mathrm{C}$ & -3.16731900 & 5.45775800 & -1.61965500 & $\mathrm{H}$ & -4.16884100 & -1.08352500 & -0.61106600 \\
\hline $\mathrm{C}$ & -4.46628800 & 5.01852800 & -1.34977200 & $\mathrm{H}$ & -6.45865800 & -2.02372900 & -0.62582600 \\
\hline $\mathrm{C}$ & -4.66444900 & 3.79001300 & -0.71975100 & $\mathrm{H}$ & -6.81710500 & -4.44258400 & -0.16514000 \\
\hline $\mathrm{C}$ & -3.56979900 & 2.99638000 & -0.36693500 & $\mathrm{H}$ & -4.86403000 & -5.91109800 & 0.29900100 \\
\hline $\mathrm{C}$ & -2.26238200 & 3.42323200 & -0.63631800 & $\mathrm{H}$ & -2.58096200 & -4.98033200 & 0.29536400 \\
\hline $\mathrm{C}$ & 1.43643000 & 3.05285300 & 1.35520400 & $\mathrm{H}$ & -0.01522600 & -4.67827500 & -1.19598500 \\
\hline $\mathrm{C}$ & 2.15000600 & 3.78227800 & 2.30666400 & $\mathrm{H}$ & 1.41654100 & -6.29295100 & -0.01536300 \\
\hline $\mathrm{C}$ & 1.55784300 & 4.86374000 & 2.96408800 & $\mathrm{H}$ & 1.81123100 & -6.06759900 & 2.42929000 \\
\hline $\mathrm{C}$ & 0.24047500 & 5.21462800 & 2.66815900 & $\mathrm{H}$ & 0.76219400 & -4.18993800 & 3.68098400 \\
\hline $\mathrm{C}$ & -0.47973900 & 4.49593500 & 1.71114100 & $\mathrm{H}$ & -0.64594200 & -2.54223700 & 2.49384900 \\
\hline $\mathrm{C}$ & 0.11467100 & 3.41699700 & 1.03964900 & $\mathrm{C}$ & -2.32723000 & 0.23615800 & 1.67889600 \\
\hline $\mathrm{C}$ & -4.32023600 & -2.14253900 & -0.42588200 & $\mathrm{O}$ & -3.07961300 & 0.34418900 & 2.55735200 \\
\hline $\mathrm{C}$ & -5.61007800 & -2.67375000 & -0.42978100 & $\mathrm{H}$ & 0.26730200 & -0.27545100 & 1.52398200 \\
\hline $\mathrm{C}$ & -5.81137100 & -4.03096400 & -0.17027500 & $\mathrm{H}$ & -2.40897000 & 0.24707800 & -0.75786500 \\
\hline $\mathrm{C}$ & -4.71549000 & -4.85493500 & 0.09019900 & $\mathrm{H}$ & 1.26718500 & 0.07808300 & -0.98162800 \\
\hline
\end{tabular}




\begin{tabular}{|c|c|c|c|c|c|c|c|}
\hline $\mathrm{C}$ & 5.07600200 & 1.13098100 & 0.45635400 & $\mathrm{C}$ & -3.40926900 & 3.14958600 & -0.44901400 \\
\hline $\mathrm{H}$ & 4.33608700 & 1.93693900 & 0.39365400 & $\mathrm{C}$ & -2.07288300 & 3.50438400 & -0.67608200 \\
\hline $\mathrm{H}$ & 5.80244700 & 1.41053900 & 1.23390100 & $\mathrm{C}$ & 1.54263600 & 3.05133100 & 1.42532900 \\
\hline $\mathrm{C}$ & 5.79094200 & 0.96763900 & -0.90156500 & $\mathrm{C}$ & 2.23824800 & 3.76867800 & 2.39908000 \\
\hline $\mathrm{H}$ & 6.28890200 & 1.90893300 & -1.17359900 & $\mathrm{C}$ & 1.63592400 & 4.84671300 & 3.05238300 \\
\hline $\mathrm{C}$ & 4.37756900 & -0.19050400 & 0.83734500 & $\mathrm{C}$ & 0.32747700 & 5.20485000 & 2.72767100 \\
\hline $\mathrm{H}$ & 3.85998700 & -0.07200900 & 1.79904300 & $\mathrm{C}$ & -0.37246100 & 4.49733000 & 1.74757400 \\
\hline $\mathrm{C}$ & 3.31857900 & -0.55899600 & -0.23621300 & $\mathrm{C}$ & 0.23008500 & 3.42020200 & 1.08000100 \\
\hline $\mathrm{H}$ & 2.91680400 & -1.55905000 & 0.03818500 & $\mathrm{C}$ & -4.47430300 & -1.78533200 & -0.59232200 \\
\hline $\mathrm{C}$ & 4.74628200 & 0.60291200 & -1.97755400 & $\mathrm{C}$ & -5.79782300 & -2.20379600 & -0.73680400 \\
\hline $\mathrm{H}$ & 5.23094300 & 0.51051400 & -2.96075200 & $\mathrm{C}$ & -6.12030400 & -3.55856700 & -0.64119300 \\
\hline $\mathrm{H}$ & 3.99398300 & 1.39648200 & -2.05405000 & $\mathrm{C}$ & -5.11225500 & -4.49465000 & -0.40249500 \\
\hline $\mathrm{C}$ & 4.05459000 & -0.72136800 & -1.59863300 & $\mathrm{C}$ & -3.78748100 & -4.07945200 & -0.26184500 \\
\hline $\mathrm{H}$ & 3.31309300 & -0.98099200 & -2.36851300 & $\mathrm{C}$ & -3.45264700 & -2.71912700 & -0.35910400 \\
\hline $\mathrm{C}$ & 5.10591900 & -1.84571500 & -1.49405100 & $\mathrm{C}$ & -0.17286500 & -4.54483100 & 0.09743200 \\
\hline $\mathrm{H}$ & 4.61868000 & -2.79827900 & -1.23934800 & $\mathrm{C}$ & 0.44881100 & -5.52403500 & 0.87712400 \\
\hline $\mathrm{H}$ & 5.60315000 & -1.99245000 & -2.46413400 & $\mathrm{C}$ & 0.37933300 & -5.46200300 & 2.26899400 \\
\hline $\mathrm{C}$ & 6.84067000 & -0.15916500 & -0.79838900 & $\mathrm{C}$ & -0.31115300 & -4.41298500 & 2.88151500 \\
\hline $\mathrm{H}$ & 7.59969600 & 0.09965600 & -0.04594900 & $\mathrm{C}$ & -0.92130200 & -3.42948000 & 2.10420500 \\
\hline $\mathrm{H}$ & 7.36783800 & -0.27425300 & -1.75668800 & $\mathrm{C}$ & -0.86547500 & -3.48418800 & 0.70076200 \\
\hline $\mathrm{C}$ & 5.43045600 & -1.31402500 & 0.94109900 & $\mathrm{H}$ & 0.24581300 & 3.12504400 & -2.32878900 \\
\hline $\mathrm{H}$ & 6.16229900 & -1.07545100 & 1.72663800 & $\mathrm{H}$ & 1.44865700 & 2.31011700 & -1.30594700 \\
\hline $\mathrm{H}$ & 4.94942300 & -2.25901600 & 1.23174000 & $\mathrm{H}$ & -0.82494200 & 0.97102100 & -2.86529100 \\
\hline $\mathrm{C}$ & 6.14747200 & -1.48399100 & -0.41479400 & $\mathrm{H}$ & 0.91805500 & 0.80145600 & -3.12816300 \\
\hline $\mathrm{H}$ & 6.89666100 & -2.28463200 & -0.34017900 & $\mathrm{H}$ & -0.97539500 & -3.23831300 & -2.37104700 \\
\hline $\mathrm{O}$ & 2.29489100 & 0.38991600 & -0.28223300 & $\mathrm{H}$ & -1.77618600 & -1.67361300 & -2.59089300 \\
\hline \multirow[t]{2}{*}{$\mathrm{H}$} & 0.79674400 & -0.02522500 & 0.99825500 & $\mathrm{H}$ & 1.01836100 & -2.09747100 & -1.39929700 \\
\hline & & & & $\mathrm{H}$ & 0.69235700 & -1.42043200 & -2.99157700 \\
\hline 27 & & & & $\mathrm{H}$ & -0.77031100 & 5.03904900 & -1.47380900 \\
\hline $\mathrm{Ru}$ & -1.18328000 & 0.11002600 & 0.26501300 & $\mathrm{H}$ & -2.60558500 & 6.51499300 & -2.19931900 \\
\hline $\mathrm{P}$ & -0.69157100 & 2.39686000 & -0.15161800 & $\mathrm{H}$ & -4.97225700 & 5.85981500 & -1.79602900 \\
\hline $\mathrm{P}$ & -1.70451400 & -2.13814900 & -0.24331100 & $\mathrm{H}$ & -5.47858000 & 3.70422800 & -0.66270200 \\
\hline $\mathrm{N}$ & 0.24507500 & -0.14215600 & -1.35674900 & $\mathrm{H}$ & -3.63647600 & 2.20808000 & 0.03795100 \\
\hline $\mathrm{C}$ & 0.41199700 & 2.27963300 & -1.65364800 & $\mathrm{H}$ & 2.02413100 & 2.20084200 & 0.94627100 \\
\hline $\mathrm{C}$ & 0.15048100 & 0.93402100 & -2.34575300 & $\mathrm{H}$ & 3.25542500 & 3.47839200 & 2.64941100 \\
\hline $\mathrm{C}$ & -1.04782700 & -2.21636700 & -1.97794500 & $\mathrm{H}$ & 2.18120400 & 5.39977800 & 3.81275900 \\
\hline $\mathrm{C}$ & 0.30399700 & -1.47593600 & -1.96241600 & $\mathrm{H}$ & -0.15397200 & 6.03715600 & 3.23460400 \\
\hline $\mathrm{C}$ & -1.79898700 & 4.73121000 & -1.30660800 & $\mathrm{H}$ & -1.38830500 & 4.79139800 & 1.50443200 \\
\hline $\mathrm{C}$ & -2.83525200 & 5.57235100 & -1.70963500 & $\mathrm{H}$ & -4.22747300 & -0.73040500 & -0.65642900 \\
\hline $\mathrm{C}$ & -4.16416900 & 5.20447300 & -1.48196800 & $\mathrm{H}$ & -6.57767200 & -1.46832700 & -0.91567200 \\
\hline $\mathrm{C}$ & -4.44800000 & 3.99516800 & -0.84778600 & $\mathrm{H}$ & -7.15209300 & -3.88312500 & -0.74678000 \\
\hline
\end{tabular}




\begin{tabular}{|c|c|c|c|c|c|c|c|}
\hline $\mathrm{H}$ & -5.35597900 & -5.55076200 & -0.32204600 & $\mathrm{C}$ & 3.33313900 & -1.79890400 & 0.55357600 \\
\hline $\mathrm{H}$ & -3.01488200 & -4.81752400 & -0.06988100 & $\mathrm{H}$ & 3.98212300 & -2.26651200 & 1.30682900 \\
\hline $\mathrm{H}$ & -0.10929600 & -4.61598000 & -0.98374300 & $\mathrm{H}$ & 2.71578100 & -2.59593800 & 0.11884900 \\
\hline $\mathrm{H}$ & 0.98758800 & -6.33422100 & 0.39293600 & $\mathrm{C}$ & 4.17815300 & -1.11847900 & -0.54165700 \\
\hline $\mathrm{H}$ & 0.86348700 & -6.22373100 & 2.87402900 & $\mathrm{H}$ & 4.81461800 & -1.86734700 & -1.03150200 \\
\hline $\mathrm{H}$ & -0.36807200 & -4.35563500 & 3.96512900 & $\mathrm{C}$ & 2.42709400 & -0.74481200 & 1.24160200 \\
\hline $\mathrm{H}$ & -1.44235200 & -2.60801700 & 2.58952300 & $\mathrm{H}$ & 1.81677600 & -1.22526800 & 2.01591000 \\
\hline $\mathrm{C}$ & -2.42858800 & 0.36635100 & 1.65829700 & $\mathrm{C}$ & 3.32268900 & 0.34956100 & 1.87955400 \\
\hline $\mathrm{O}$ & -3.23360300 & 0.52133000 & 2.48370800 & $\mathrm{H}$ & 3.97214800 & -0.10968800 & 2.63755100 \\
\hline $\mathrm{H}$ & 0.17087800 & -0.60254700 & 1.50111600 & $\mathrm{H}$ & 2.69730200 & 1.09014100 & 2.39488000 \\
\hline $\mathrm{H}$ & -2.33045900 & 0.38054000 & -0.80580000 & $\mathrm{C}$ & 5.06018800 & -0.02284100 & 0.09412300 \\
\hline $\mathrm{H}$ & 1.82361200 & 0.13376100 & -0.64742600 & $\mathrm{H}$ & 5.67911000 & 0.45599800 & -0.67706500 \\
\hline $\mathrm{C}$ & 5.54169800 & 0.94712400 & 0.19846800 & $\mathrm{H}$ & 5.74915700 & -0.46981100 & 0.82434000 \\
\hline $\mathrm{H}$ & 4.87327900 & 1.80799700 & 0.08472800 & $\mathrm{C}$ & 4.16704200 & 1.03027300 & 0.78400700 \\
\hline $\mathrm{H}$ & 6.35076600 & 1.24780400 & 0.87963000 & $\mathrm{H}$ & 4.79579200 & 1.80834100 & 1.23690400 \\
\hline $\mathrm{C}$ & 6.12411200 & 0.54132800 & -1.17215500 & $\mathrm{C}$ & 3.22328700 & 1.66721400 & -0.25506700 \\
\hline $\mathrm{H}$ & 6.67782700 & 1.38678200 & -1.60291400 & $\mathrm{H}$ & 2.59685100 & 2.43533700 & 0.21638800 \\
\hline $\mathrm{C}$ & 4.76552100 & -0.24142300 & 0.80413300 & $\mathrm{H}$ & 3.80268400 & 2.16512000 & -1.04438400 \\
\hline $\mathrm{H}$ & 4.33821400 & 0.04731800 & 1.77341300 & $\mathrm{C}$ & 3.23388100 & -0.48188600 & -1.58023700 \\
\hline $\mathrm{C}$ & 3.60777100 & -0.64436700 & -0.13267000 & $\mathrm{H}$ & 2.61596000 & -1.25323300 & -2.05814500 \\
\hline $\mathrm{H}$ & 3.10300500 & -1.52672300 & 0.30104200 & $\mathrm{H}$ & 3.81303600 & 0.00429300 & -2.37701300 \\
\hline $\mathrm{C}$ & 4.96616200 & 0.14624200 & -2.11316800 & $\mathrm{C}$ & 1.53320300 & -0.10166700 & 0.19902800 \\
\hline $\mathrm{H}$ & 5.35728600 & -0.12385600 & -3.10452000 & $\mathrm{C}$ & 2.32553400 & 0.57678500 & -0.89946700 \\
\hline $\mathrm{H}$ & 4.28501600 & 0.99400500 & -2.24887500 & $\mathrm{H}$ & 1.65005600 & 1.02600500 & -1.62590200 \\
\hline $\mathrm{C}$ & 4.19331800 & -1.04449600 & -1.51097700 & $\mathrm{C}$ & 0.18613200 & -0.16590600 & 0.30239200 \\
\hline $\mathrm{H}$ & 3.36689500 & -1.32512900 & -2.17848600 & $\mathrm{H}$ & -0.22937000 & -0.69827900 & 1.15674100 \\
\hline $\mathrm{C}$ & 5.14528100 & -2.24558000 & -1.32848600 & $\mathrm{C}$ & -0.82331600 & 0.43536100 & -0.60003000 \\
\hline $\mathrm{H}$ & 4.59887500 & -3.10494700 & -0.91354600 & $\mathrm{C}$ & -2.27208400 & 0.15546200 & -0.25940500 \\
\hline $\mathrm{H}$ & 5.54129700 & -2.56198100 & -2.30380500 & $\mathrm{C}$ & -2.78209500 & -1.15782600 & -0.29575100 \\
\hline $\mathrm{C}$ & 7.07516300 & -0.66125300 & -0.99275500 & $\mathrm{C}$ & -3.12202800 & 1.23992900 & 0.04994100 \\
\hline $\mathrm{H}$ & 7.91387600 & -0.38524400 & -0.33771100 & $\mathrm{C}$ & -4.13559700 & -1.36594700 & -0.00470600 \\
\hline $\mathrm{H}$ & 7.50825400 & -0.94852100 & -1.96161400 & $\mathrm{C}$ & -4.46040400 & 0.98088000 & 0.35525800 \\
\hline $\mathrm{C}$ & 5.71891700 & -1.44181800 & 0.98215400 & $\mathrm{C}$ & -4.98871000 & -0.31450200 & 0.33651000 \\
\hline $\mathrm{H}$ & 6.52975700 & -1.17420500 & 1.67415800 & $\mathrm{H}$ & -4.53231500 & -2.37843100 & -0.05222000 \\
\hline $\mathrm{H}$ & 5.18362200 & -2.28974000 & 1.43269800 & $\mathrm{H}$ & -5.11140700 & 1.81629900 & 0.60662200 \\
\hline $\mathrm{C}$ & 6.30229500 & -1.85213500 & -0.38635000 & $\mathrm{O}$ & -0.55134400 & 1.16436100 & -1.55255000 \\
\hline $\mathrm{H}$ & 6.98073100 & -2.70654900 & -0.25751500 & $\mathrm{C}$ & -2.61691200 & 2.66693800 & 0.06200300 \\
\hline $\mathrm{O}$ & 2.69290100 & 0.43386100 & -0.22245000 & $\mathrm{H}$ & -1.72981400 & 2.78060500 & 0.69672700 \\
\hline \multirow[t]{2}{*}{$\mathrm{H}$} & 0.44059100 & 0.12424500 & 1.40269200 & $\mathrm{H}$ & -2.32715700 & 2.99032700 & -0.94239800 \\
\hline & & & & $\mathrm{H}$ & -3.38852800 & 3.34587200 & 0.43808700 \\
\hline cd1 & & & & $\mathrm{C}$ & -6.43659500 & -0.56588800 & 0.68846600 \\
\hline
\end{tabular}




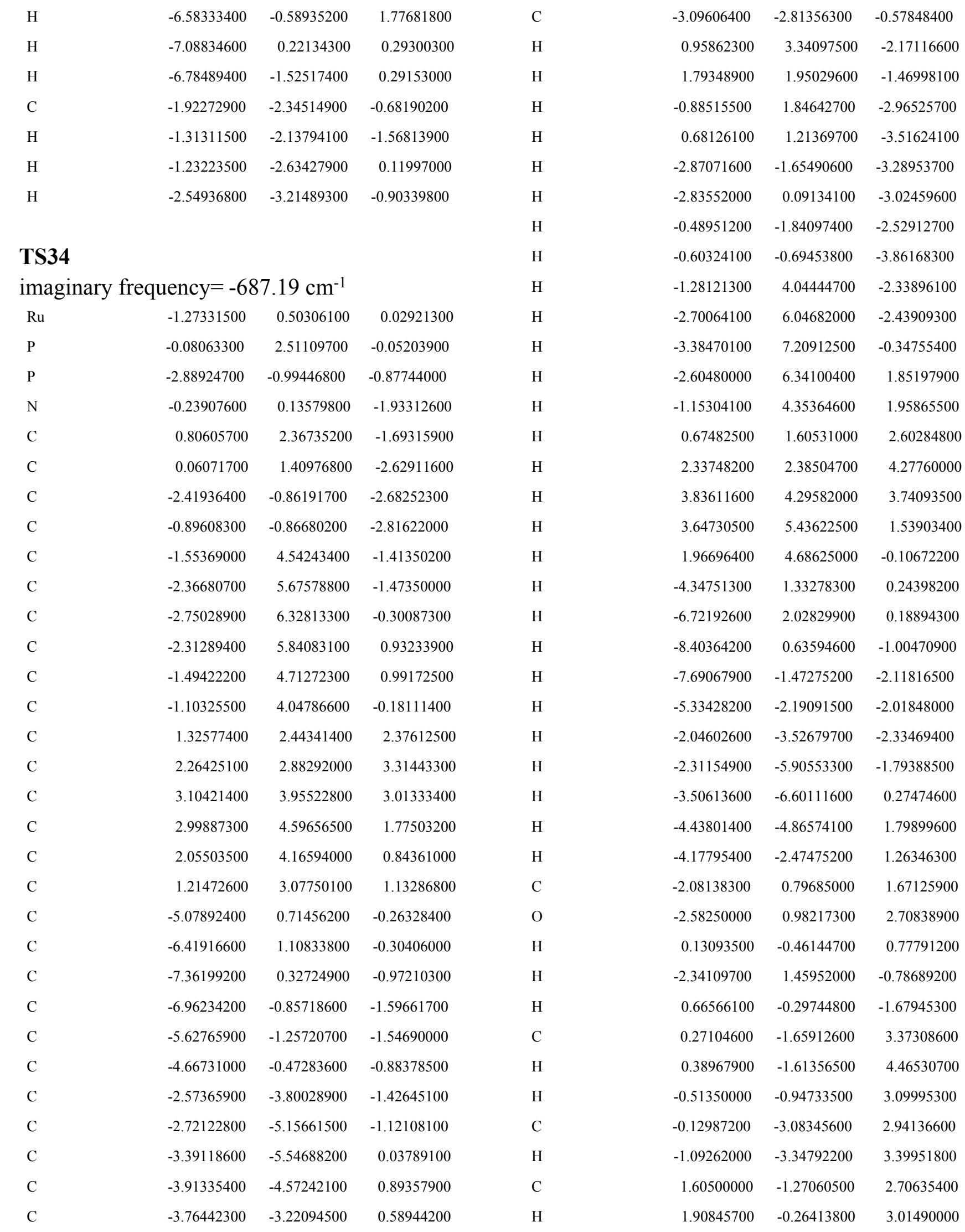




\begin{tabular}{|c|c|c|c|c|c|c|c|}
\hline $\mathrm{C}$ & 2.68918800 & -2.28778700 & 3.17359800 & \multirow{2}{*}{\multicolumn{2}{|c|}{ 6a (B3LYP-D3 optimized) }} & & \\
\hline $\mathrm{H}$ & 2.77413200 & -2.24455600 & 4.26903300 & & & & \\
\hline $\mathrm{H}$ & 3.66529600 & -2.01311600 & 2.75761700 & $\mathrm{Ru}$ & -0.31333300 & 0.41927200 & 0.06862700 \\
\hline $\mathrm{C}$ & 0.95061400 & -4.08880600 & 3.38574600 & $\mathrm{P}$ & -2.61392400 & 0.14316900 & -0.31261300 \\
\hline $\mathrm{H}$ & 0.66126100 & -5.10835100 & 3.09376800 & $\mathrm{P}$ & 1.96316500 & 0.93325100 & -0.24420300 \\
\hline $\mathrm{H}$ & 1.05113100 & -4.08473100 & 4.48074100 & $\mathrm{~N}$ & -0.27123800 & 0.36823700 & -2.04845300 \\
\hline $\mathrm{C}$ & 2.29350600 & -3.70971000 & 2.72894400 & $\mathrm{C}$ & -2.69217500 & -0.03206100 & -2.16103100 \\
\hline $\mathrm{H}$ & 3.07255900 & -4.41957500 & 3.03979500 & $\mathrm{C}$ & -1.50468100 & 0.73670100 & -2.75118100 \\
\hline $\mathrm{C}$ & 2.14507300 & -3.74843400 & 1.19388700 & $\mathrm{C}$ & 2.18378300 & 0.48699300 & -2.03035300 \\
\hline $\mathrm{H}$ & 3.10308500 & -3.51999600 & 0.71333400 & $\mathrm{C}$ & 0.89713800 & 0.91349700 & -2.74298200 \\
\hline $\mathrm{H}$ & 1.84569700 & -4.75570200 & 0.87131100 & $\mathrm{C}$ & -4.42019800 & -2.05834100 & -0.41339300 \\
\hline $\mathrm{C}$ & -0.26773900 & -3.11379100 & 1.40848500 & $\mathrm{C}$ & -5.03563700 & -3.19327300 & 0.11745500 \\
\hline $\mathrm{H}$ & -1.04706600 & -2.41741100 & 1.09624700 & $\mathrm{C}$ & -4.72641700 & -3.61766500 & 1.41140100 \\
\hline $\mathrm{H}$ & -0.56640800 & -4.11643800 & 1.07527400 & $\mathrm{C}$ & -3.80091800 & -2.90178300 & 2.17392700 \\
\hline $\mathrm{C}$ & 1.56125100 & -1.34399700 & 1.17117400 & $\mathrm{C}$ & -3.18009900 & -1.77119700 & 1.64264900 \\
\hline $\mathrm{C}$ & 1.06020000 & -2.72828500 & 0.73193200 & $\mathrm{C}$ & -3.48414800 & -1.33698600 & 0.34348300 \\
\hline $\mathrm{H}$ & 0.96777200 & -2.76301800 & -0.35487400 & $\mathrm{C}$ & -3.34185600 & 2.58923500 & 0.89320800 \\
\hline $\mathrm{C}$ & 2.66256400 & -0.73461500 & 0.53197300 & $\mathrm{C}$ & -4.22270400 & 3.62495900 & 1.21544300 \\
\hline $\mathrm{H}$ & 3.33417200 & -0.16865100 & 1.17104400 & $\mathrm{C}$ & -5.53746300 & 3.59810700 & 0.74960900 \\
\hline $\mathrm{C}$ & 3.03475200 & -0.86630700 & -0.83581300 & $\mathrm{C}$ & -5.97573100 & 2.52675700 & -0.03482400 \\
\hline $\mathrm{C}$ & 4.49201700 & -0.67942000 & -1.22386000 & $\mathrm{C}$ & -5.09901500 & 1.49125000 & -0.35286800 \\
\hline $\mathrm{C}$ & 5.24078800 & 0.47287200 & -0.90305800 & $\mathrm{C}$ & -3.76998400 & 1.51614200 & 0.10100700 \\
\hline $\mathrm{C}$ & 5.11172800 & -1.71681100 & -1.97220300 & $\mathrm{C}$ & 4.50305600 & -0.31974600 & 0.03294500 \\
\hline $\mathrm{C}$ & 6.57589300 & 0.56710400 & -1.32452000 & $\mathrm{C}$ & 5.55425800 & -0.85114000 & 0.78167900 \\
\hline $\mathrm{C}$ & 6.44918600 & -1.58593600 & -2.34720700 & $\mathrm{C}$ & 5.44805000 & -0.94844300 & 2.16955300 \\
\hline $\mathrm{C}$ & 7.20523000 & -0.44965900 & -2.03724200 & $\mathrm{C}$ & 4.27985800 & -0.52308600 & 2.80784300 \\
\hline $\mathrm{H}$ & 7.13421900 & 1.47070200 & -1.08443800 & $\mathrm{C}$ & 3.22813300 & 0.00764600 & 2.06191100 \\
\hline $\mathrm{H}$ & 6.91509800 & -2.39569400 & -2.90659700 & $\mathrm{C}$ & 3.33496900 & 0.12483000 & 0.66719200 \\
\hline $\mathrm{O}$ & 2.23077100 & -1.19326900 & -1.75383800 & $\mathrm{C}$ & 3.77208600 & 3.07328700 & -0.60578400 \\
\hline $\mathrm{C}$ & 4.37892800 & -2.98021500 & -2.37298400 & $\mathrm{C}$ & 4.17521000 & 4.40701100 & -0.58941200 \\
\hline $\mathrm{H}$ & 3.60102500 & -2.76846000 & -3.11118700 & $\mathrm{C}$ & 3.29462300 & 5.39902500 & -0.14764100 \\
\hline $\mathrm{H}$ & 5.08061300 & -3.70787100 & -2.79446300 & $\mathrm{C}$ & 2.01452200 & 5.04671300 & 0.27938300 \\
\hline $\mathrm{H}$ & 3.87493500 & -3.44991900 & -1.52118400 & $\mathrm{C}$ & 1.60926300 & 3.70937500 & 0.25928200 \\
\hline $\mathrm{C}$ & 8.65059500 & -0.33889800 & -2.46373300 & $\mathrm{C}$ & 2.48026100 & 2.70881800 & -0.18937200 \\
\hline $\mathrm{H}$ & 9.27549700 & -1.08804000 & -1.95994900 & $\mathrm{H}$ & -2.56244000 & -1.09784700 & -2.37331600 \\
\hline $\mathrm{H}$ & 8.76540700 & -0.50153900 & -3.54273700 & $\mathrm{H}$ & -3.64780000 & 0.31004000 & -2.57108800 \\
\hline $\mathrm{H}$ & 9.06258500 & 0.64789700 & -2.22812600 & $\mathrm{H}$ & -1.42290400 & 0.48400000 & -3.82253400 \\
\hline $\mathrm{C}$ & 4.68040500 & 1.64886900 & -0.13089600 & $\mathrm{H}$ & -1.69115500 & 1.82445600 & -2.69959700 \\
\hline $\mathrm{H}$ & 3.60326000 & 1.76608200 & -0.25758700 & $\mathrm{H}$ & 3.06656300 & 0.96081000 & -2.47246200 \\
\hline $\mathrm{H}$ & 4.85771500 & 1.55146100 & 0.94843300 & $\mathrm{H}$ & 2.29914800 & -0.60169300 & -2.07796800 \\
\hline $\mathrm{H}$ & 5.16385000 & 2.57916300 & -0.44906900 & $\mathrm{H}$ & 0.85874800 & 2.01735400 & -2.80053700 \\
\hline
\end{tabular}




\begin{tabular}{|c|c|c|c|c|c|c|c|}
\hline $\mathrm{H}$ & 0.92517800 & 0.54852000 & -3.78429600 & $\mathrm{H}$ & -0.80221300 & -4.12517600 & 0.66156400 \\
\hline $\mathrm{H}$ & -4.66146300 & -1.75038500 & -1.42650400 & $\mathrm{H}$ & -2.03462500 & -3.57746300 & -0.50084400 \\
\hline $\mathrm{H}$ & -5.75075300 & -3.74917000 & -0.48301500 & & & & \\
\hline $\mathrm{H}$ & -5.20059400 & -4.50538400 & 1.82102500 & \multicolumn{4}{|c|}{ TS3a (B3LYP-D3 optimized) } \\
\hline $\mathrm{H}$ & -3.55154500 & -3.22988200 & 3.17932600 & \multicolumn{4}{|c|}{ imaginary frequency $=-975.98 \mathrm{~cm}^{-1}$} \\
\hline $\mathrm{H}$ & -2.44558500 & -1.23395600 & 2.23408100 & $\mathrm{Ru}$ & -0.35001600 & 0.40173000 & 0.09269100 \\
\hline $\mathrm{H}$ & -2.31937100 & 2.60875900 & 1.25565100 & $\mathrm{P}$ & -2.63955500 & 0.01893200 & -0.29439900 \\
\hline $\mathrm{H}$ & -3.87846600 & 4.45164000 & 1.83119600 & $\mathrm{P}$ & 1.91036500 & 0.98557300 & -0.22836300 \\
\hline $\mathrm{H}$ & -6.22149500 & 4.40498800 & 0.99880300 & $\mathrm{~N}$ & -0.29782500 & 0.30164200 & -2.08297600 \\
\hline $\mathrm{H}$ & -7.00089400 & 2.49708700 & -0.39437300 & $\mathrm{C}$ & -2.68394800 & -0.24310500 & -2.13537500 \\
\hline $\mathrm{H}$ & -5.45590000 & 0.65314300 & -0.94575700 & $\mathrm{C}$ & -1.57350700 & 0.60798500 & -2.75851700 \\
\hline $\mathrm{H}$ & 4.59419400 & -0.27052200 & -1.04758600 & $\mathrm{C}$ & 2.14083000 & 0.58242400 & -2.02659800 \\
\hline $\mathrm{H}$ & 6.45147800 & -1.19896800 & 0.27690000 & $\mathrm{C}$ & 0.84284700 & 0.95954200 & -2.74566400 \\
\hline $\mathrm{H}$ & 6.26726100 & -1.36294100 & 2.75109500 & $\mathrm{C}$ & -4.38665700 & -2.22910200 & -0.31516300 \\
\hline $\mathrm{H}$ & 4.18534700 & -0.60737300 & 3.88714900 & $\mathrm{C}$ & -4.98433000 & -3.34945900 & 0.26409900 \\
\hline $\mathrm{H}$ & 2.31987200 & 0.32597700 & 2.56449900 & $\mathrm{C}$ & -4.68599900 & -3.69960700 & 1.58251600 \\
\hline $\mathrm{H}$ & 4.47415400 & 2.30966200 & -0.92925200 & $\mathrm{C}$ & -3.78808100 & -2.92546900 & 2.32080800 \\
\hline $\mathrm{H}$ & 5.17716600 & 4.67246400 & -0.91629600 & $\mathrm{C}$ & -3.18447200 & -1.80933300 & 1.74164700 \\
\hline $\mathrm{H}$ & 3.60898300 & 6.43926100 & -0.13290600 & $\mathrm{C}$ & -3.47991300 & -1.44884300 & 0.41849100 \\
\hline $\mathrm{H}$ & 1.32606200 & 5.81113100 & 0.62993400 & $\mathrm{C}$ & -3.45777700 & 2.52023500 & 0.73321100 \\
\hline $\mathrm{H}$ & 0.61306000 & 3.43829700 & 0.59147500 & $\mathrm{C}$ & -4.37584100 & 3.54503200 & 0.97709300 \\
\hline $\mathrm{C}$ & -0.32101200 & 0.31480800 & 1.94206600 & $\mathrm{C}$ & -5.68675500 & 3.43784300 & 0.51218400 \\
\hline $\mathrm{O}$ & -0.32400400 & 0.27047900 & 3.10938400 & $\mathrm{C}$ & -6.08367200 & 2.29698500 & -0.19219000 \\
\hline $\mathrm{H}$ & -0.59209600 & 1.95471400 & 0.17311100 & $\mathrm{C}$ & -5.16995000 & 1.27238800 & -0.43146700 \\
\hline $\mathrm{C}$ & 3.25586500 & -3.46863600 & 0.86910000 & $\mathrm{C}$ & -3.84431000 & 1.37730600 & 0.02148600 \\
\hline $\mathrm{C}$ & 1.93206700 & -3.07996800 & 0.67459900 & $\mathrm{C}$ & 4.46506200 & -0.24295100 & 0.01881200 \\
\hline $\mathrm{C}$ & 1.38511100 & -3.02663700 & -0.61395400 & $\mathrm{C}$ & 5.52551200 & -0.76715500 & 0.75900800 \\
\hline $\mathrm{C}$ & 2.19443700 & -3.34570800 & -1.70837600 & $\mathrm{C}$ & 5.42822500 & -0.87343700 & 2.14674300 \\
\hline $\mathrm{C}$ & 3.52613700 & -3.72557300 & -1.51673600 & $\mathrm{C}$ & 4.25869600 & -0.46704200 & 2.79438900 \\
\hline $\mathrm{C}$ & 4.05753500 & -3.79528100 & -0.22812700 & $\mathrm{C}$ & 3.19638000 & 0.05443600 & 2.05754900 \\
\hline $\mathrm{H}$ & 3.67212000 & -3.48619300 & 1.87184800 & $\mathrm{C}$ & 3.29567700 & 0.18231300 & 0.66315500 \\
\hline $\mathrm{H}$ & 1.31643800 & -2.80446900 & 1.52857600 & $\mathrm{C}$ & 3.67095000 & 3.17201900 & -0.53601000 \\
\hline $\mathrm{H}$ & 1.76163100 & -3.29316600 & -2.70229000 & $\mathrm{C}$ & 4.04508200 & 4.51307400 & -0.48032500 \\
\hline $\mathrm{H}$ & 4.14623500 & -3.97094600 & -2.37583300 & $\mathrm{C}$ & 3.14451800 & 5.47175300 & -0.00598200 \\
\hline $\mathrm{H}$ & 5.09394300 & -4.08567500 & -0.07733000 & $\mathrm{C}$ & 1.87367700 & 5.07946900 & 0.41403800 \\
\hline $\mathrm{C}$ & -0.08671800 & -2.68345100 & -0.81168400 & $\mathrm{C}$ & 1.49662400 & 3.73504600 & 0.35373300 \\
\hline $\mathrm{H}$ & -0.32039100 & -1.82626900 & -0.11978500 & $\mathrm{C}$ & 2.38802300 & 2.76853100 & -0.12815300 \\
\hline $\mathrm{O}$ & -0.40103200 & -2.33694000 & -2.13306900 & $\mathrm{H}$ & -2.44018500 & -1.29739700 & -2.30594100 \\
\hline $\mathrm{H}$ & -0.24888300 & -1.33813200 & -2.20887700 & $\mathrm{H}$ & -3.66309500 & -0.01226800 & -2.56650900 \\
\hline $\mathrm{C}$ & -0.98321500 & -3.84942600 & -0.38221200 & $\mathrm{H}$ & -1.49713700 & 0.36983400 & -3.83139600 \\
\hline $\mathrm{H}$ & -0.76838100 & -4.71741300 & -1.01533000 & $\mathrm{H}$ & -1.80255400 & 1.67982400 & -2.67357000 \\
\hline
\end{tabular}




\begin{tabular}{|c|c|c|c|c|c|c|c|}
\hline $\mathrm{H}$ & 3.00273400 & 1.10018400 & -2.45973400 & $\mathrm{H}$ & -0.14537600 & -0.91410700 & -2.21862700 \\
\hline $\mathrm{H}$ & 2.29908700 & -0.49919800 & -2.09662200 & $\mathrm{C}$ & -0.78196100 & -3.65133800 & -0.45185900 \\
\hline $\mathrm{H}$ & 0.70714600 & 2.05110000 & -2.74367100 & $\mathrm{H}$ & -0.58641100 & -4.55607100 & -1.03829500 \\
\hline $\mathrm{H}$ & 0.90069000 & 0.63697900 & -3.79734700 & $\mathrm{H}$ & -0.64063300 & -3.88250200 & 0.60977100 \\
\hline $\mathrm{H}$ & -4.61668100 & -1.98024700 & -1.34700400 & $\mathrm{H}$ & -1.81966200 & -3.35095400 & -0.61187600 \\
\hline $\mathrm{H}$ & -5.67651700 & -3.95231800 & -0.31754600 & & & & \\
\hline $\mathrm{H}$ & -5.14671600 & -4.57590200 & 2.03028300 & \multicolumn{4}{|c|}{ 6b (B3LYP-D3 optimized) } \\
\hline $\mathrm{H}$ & -3.54684800 & -3.19692000 & 3.34486200 & $\mathrm{Ru}$ & -0.33013200 & 0.48407100 & 0.05864400 \\
\hline $\mathrm{H}$ & -2.47059400 & -1.22583800 & 2.31459000 & $\mathrm{P}$ & 1.83268000 & 1.34960200 & 0.34961600 \\
\hline $\mathrm{H}$ & -2.43774900 & 2.60306300 & 1.09319500 & $\mathrm{P}$ & -2.59438700 & -0.03159800 & 0.39373800 \\
\hline $\mathrm{H}$ & -4.06342600 & 4.42616100 & 1.53121400 & $\mathrm{~N}$ & -0.41380900 & 0.85976100 & 2.20855500 \\
\hline $\mathrm{H}$ & -6.39979200 & 4.23607300 & 0.70040500 & $\mathrm{C}$ & 1.94153600 & 1.55429500 & 2.20636500 \\
\hline $\mathrm{H}$ & -7.10561000 & 2.20464500 & -0.55021100 & $\mathrm{C}$ & 0.53189700 & 1.83576800 & 2.73782900 \\
\hline $\mathrm{H}$ & -5.49560100 & 0.38063500 & -0.96050200 & $\mathrm{C}$ & -2.70868700 & -0.01177400 & 2.25504600 \\
\hline $\mathrm{H}$ & 4.54962400 & -0.18788400 & -1.06180200 & $\mathrm{C}$ & -1.74532200 & 1.06134700 & 2.77428300 \\
\hline $\mathrm{H}$ & 6.42210800 & -1.10501700 & 0.24687800 & $\mathrm{C}$ & 3.26771700 & -0.38926500 & -1.29550600 \\
\hline $\mathrm{H}$ & 6.25499400 & -1.28318800 & 2.72081600 & $\mathrm{C}$ & 4.37360300 & -1.13879800 & -1.69545100 \\
\hline $\mathrm{H}$ & 4.17090000 & -0.56037800 & 3.87348000 & $\mathrm{C}$ & 5.52776100 & -1.15875700 & -0.91074900 \\
\hline $\mathrm{H}$ & 2.28636300 & 0.35797200 & 2.56660100 & $\mathrm{C}$ & 5.57706200 & -0.42131100 & 0.27446700 \\
\hline $\mathrm{H}$ & 4.38845300 & 2.43271900 & -0.88192500 & $\mathrm{C}$ & 4.47602500 & 0.34041400 & 0.67034200 \\
\hline $\mathrm{H}$ & 5.04010200 & 4.81014900 & -0.80100700 & $\mathrm{C}$ & 3.31008400 & 0.36395500 & -0.11209200 \\
\hline $\mathrm{H}$ & 3.43672300 & 6.51750500 & 0.03997100 & $\mathrm{C}$ & 1.20047600 & 4.02147800 & -0.20310900 \\
\hline $\mathrm{H}$ & 1.17097200 & 5.81804600 & 0.79071400 & $\mathrm{C}$ & 1.45623900 & 5.33124300 & -0.60794700 \\
\hline $\mathrm{H}$ & 0.50779100 & 3.43106500 & 0.67972100 & $\mathrm{C}$ & 2.71391600 & 5.68002700 & -1.10672500 \\
\hline $\mathrm{C}$ & -0.37712100 & 0.41682400 & 1.96081300 & $\mathrm{C}$ & 3.71531900 & 4.71195500 & -1.19348300 \\
\hline $\mathrm{O}$ & -0.39572700 & 0.44399500 & 3.12701000 & $\mathrm{C}$ & 3.46665900 & 3.40226700 & -0.77645600 \\
\hline $\mathrm{H}$ & -0.68789000 & 1.94395300 & 0.07175100 & $\mathrm{C}$ & 2.20670900 & 3.04374900 & -0.27519100 \\
\hline $\mathrm{C}$ & 3.42798700 & -3.50565500 & 0.83786800 & $\mathrm{C}$ & -2.60029400 & -2.82287900 & 0.37894200 \\
\hline $\mathrm{C}$ & 2.12184700 & -3.07188200 & 0.62006000 & $\mathrm{C}$ & -3.01304900 & -4.09161600 & -0.02539400 \\
\hline $\mathrm{C}$ & 1.62303100 & -2.92888700 & -0.68111200 & $\mathrm{C}$ & -4.07504400 & -4.23532200 & -0.92258500 \\
\hline $\mathrm{C}$ & 2.46559900 & -3.20373200 & -1.76219500 & $\mathrm{C}$ & -4.72016400 & -3.10075600 & -1.41623200 \\
\hline $\mathrm{C}$ & 3.77951000 & -3.63080100 & -1.54795800 & $\mathrm{C}$ & -4.31344800 & -1.82672400 & -1.01208100 \\
\hline $\mathrm{C}$ & 4.26157200 & -3.79059000 & -0.24800700 & $\mathrm{C}$ & -3.25453800 & -1.67567000 & -0.10518900 \\
\hline $\mathrm{H}$ & 3.80555700 & -3.59716300 & 1.85236800 & $\mathrm{C}$ & -5.16952800 & 1.15089600 & 0.36761500 \\
\hline $\mathrm{H}$ & 1.48248700 & -2.83020600 & 1.46679500 & $\mathrm{C}$ & -6.13501200 & 2.04881400 & -0.08641600 \\
\hline $\mathrm{H}$ & 2.06169000 & -3.08170300 & -2.76234600 & $\mathrm{C}$ & -5.82036200 & 2.96192200 & -1.09691600 \\
\hline $\mathrm{H}$ & 4.42402700 & -3.84481700 & -2.39776700 & $\mathrm{C}$ & -4.53990700 & 2.96933500 & -1.65203400 \\
\hline $\mathrm{H}$ & 5.28332600 & -4.12094000 & -0.07830000 & $\mathrm{C}$ & -3.57059700 & 2.07366900 & -1.19438000 \\
\hline $\mathrm{C}$ & 0.16482300 & -2.53766900 & -0.94029300 & $\mathrm{C}$ & -3.87397000 & 1.15867600 & -0.17648800 \\
\hline $\mathrm{H}$ & -0.03098000 & -1.65516300 & -0.19530900 & $\mathrm{H}$ & 2.30468000 & 0.60484500 & 2.61539000 \\
\hline $\mathrm{O}$ & -0.09565800 & -2.17497000 & -2.22166900 & $\mathrm{H}$ & 2.64938700 & 2.34327600 & 2.48128300 \\
\hline
\end{tabular}




\begin{tabular}{|c|c|c|c|c|c|c|c|}
\hline $\mathrm{H}$ & 0.55753500 & 1.79612100 & 3.84232000 & $\mathrm{H}$ & 2.20157100 & -1.45717700 & 1.85692300 \\
\hline $\mathrm{H}$ & 0.24368900 & 2.87212400 & 2.47774500 & $\mathrm{O}$ & 0.16903300 & -1.56135000 & 1.47901600 \\
\hline $\mathrm{H}$ & -3.73331400 & 0.14946800 & 2.60447100 & $\mathrm{H}$ & 0.04926000 & -0.74825200 & 2.09749700 \\
\hline $\mathrm{H}$ & -2.38219900 & -1.00004200 & 2.59575500 & $\mathrm{C}$ & 1.31310600 & -3.11105400 & 2.91867700 \\
\hline $\mathrm{H}$ & -2.14820700 & 2.06306600 & 2.52913600 & $\mathrm{H}$ & 0.53228500 & -3.86271800 & 2.76183700 \\
\hline $\mathrm{H}$ & -1.71228200 & 1.00452000 & 3.87765100 & $\mathrm{H}$ & 2.25664800 & -3.62753200 & 3.12532800 \\
\hline $\mathrm{H}$ & 2.36429200 & -0.40599400 & -1.89485700 & $\mathrm{H}$ & 1.04690400 & -2.50937300 & 3.79544900 \\
\hline $\mathrm{H}$ & 4.31758000 & -1.73222300 & -2.60243300 & & & & \\
\hline $\mathrm{H}$ & 6.38324700 & -1.75562400 & -1.21560400 & \multicolumn{4}{|c|}{ TS3b (B3LYP-D3 optimized) } \\
\hline $\mathrm{H}$ & 6.47280400 & -0.43547400 & 0.89003100 & \multicolumn{4}{|c|}{ imaginary frequency $=-804.56 \mathrm{~cm}^{-1}$} \\
\hline $\mathrm{H}$ & 4.53172500 & 0.92134900 & 1.58662700 & $\mathrm{Ru}$ & -0.30451600 & 0.48517000 & 0.05254300 \\
\hline $\mathrm{H}$ & 0.21312100 & 3.74806900 & 0.15566200 & $\mathrm{P}$ & 1.88590700 & 1.27678100 & 0.33671900 \\
\hline $\mathrm{H}$ & 0.66837700 & 6.07723100 & -0.54340200 & $\mathrm{P}$ & -2.58545800 & 0.03413600 & 0.37155500 \\
\hline $\mathrm{H}$ & 2.90937400 & 6.69859500 & -1.43154200 & $\mathrm{~N}$ & -0.38258500 & 0.85988100 & 2.20591400 \\
\hline $\mathrm{H}$ & 4.69396400 & 4.97283200 & -1.58799400 & $\mathrm{C}$ & 2.00333300 & 1.46210700 & 2.19659000 \\
\hline $\mathrm{H}$ & 4.25586300 & 2.66067700 & -0.84756000 & $\mathrm{C}$ & 0.60952300 & 1.78910900 & 2.74258600 \\
\hline $\mathrm{H}$ & -1.74930400 & -2.71690000 & 1.04464800 & $\mathrm{C}$ & -2.70511400 & 0.06012300 & 2.23392000 \\
\hline $\mathrm{H}$ & -2.49514800 & -4.96901500 & 0.35313400 & $\mathrm{C}$ & -1.71293500 & 1.09901000 & 2.76758000 \\
\hline $\mathrm{H}$ & -4.39210300 & -5.22522300 & -1.23999300 & $\mathrm{C}$ & 3.26878500 & -0.48612600 & -1.32487000 \\
\hline $\mathrm{H}$ & -5.54211900 & -3.20292000 & -2.12007600 & $\mathrm{C}$ & 4.35232500 & -1.26323400 & -1.73286600 \\
\hline $\mathrm{H}$ & -4.82295600 & -0.95372000 & -1.40708100 & $\mathrm{C}$ & 5.50362500 & -1.32893600 & -0.94653200 \\
\hline $\mathrm{H}$ & -5.43297100 & 0.43051200 & 1.13731600 & $\mathrm{C}$ & 5.57221900 & -0.61041200 & 0.24913100 \\
\hline $\mathrm{H}$ & -7.13212300 & 2.03462200 & 0.34566000 & $\mathrm{C}$ & 4.49382200 & 0.17861700 & $0.6535070 c$ \\
\hline $\mathrm{H}$ & -6.57260900 & 3.66212300 & -1.45051000 & $\mathrm{C}$ & 3.33127000 & 0.24862800 & -0.13091000 \\
\hline $\mathrm{H}$ & -4.29107100 & 3.67355000 & -2.44138000 & $\mathrm{C}$ & 1.34292800 & 3.97305900 & -0.20012000 \\
\hline $\mathrm{H}$ & -2.57232700 & 2.08363200 & -1.61955600 & $\mathrm{C}$ & 1.64666100 & 5.27909800 & -0.58379000 \\
\hline $\mathrm{C}$ & -0.23132000 & 0.01688000 & -1.75197500 & $\mathrm{C}$ & 2.92308800 & 5.59413600 & -1.05639400 \\
\hline $\mathrm{O}$ & -0.17738600 & -0.26427500 & -2.88260000 & $\mathrm{C}$ & 3.89493800 & 4.59591300 & -1.13864300 \\
\hline $\mathrm{H}$ & -0.77526200 & 1.92986900 & -0.38747800 & $\mathrm{C}$ & 3.59816700 & 3.28983100 & -0.74261000 \\
\hline $\mathrm{C}$ & 3.47310500 & -4.30583300 & -0.75198400 & $\mathrm{C}$ & 2.31897200 & 2.96505400 & -0.26708000 \\
\hline $\mathrm{C}$ & 3.07135400 & -3.60034400 & 0.38057100 & $\mathrm{C}$ & -2.66266400 & -2.75395500 & 0.39415400 \\
\hline $\mathrm{C}$ & 1.81263200 & -2.98711800 & 0.43567300 & $\mathrm{C}$ & -3.10513900 & -4.01714200 & 0.00442200 \\
\hline $\mathrm{C}$ & 0.96195600 & -3.09409200 & -0.66941200 & $\mathrm{C}$ & -4.16309700 & -4.14608800 & -0.89975900 \\
\hline $\mathrm{C}$ & 1.36617100 & -3.79382000 & -1.80954900 & $\mathrm{C}$ & -4.77358500 & -3.00258300 & -1.41654300 \\
\hline $\mathrm{C}$ & 2.62046600 & -4.40358300 & -1.85557300 & $\mathrm{C}$ & -4.33696700 & -1.73369900 & -1.02775300 \\
\hline $\mathrm{H}$ & 4.45729100 & -4.76677400 & -0.77951200 & $\mathrm{C}$ & -3.28285400 & -1.59770000 & -0.11288300 \\
\hline $\mathrm{H}$ & 3.75512200 & -3.50143100 & 1.22060000 & $\mathrm{C}$ & -5.14149600 & 1.25325900 & 0.32431100 \\
\hline $\mathrm{H}$ & -0.00954000 & -2.61782100 & -0.64308500 & $\mathrm{C}$ & -6.08660600 & 2.17343900 & -0.12760500 \\
\hline $\mathrm{H}$ & 0.69556400 & -3.85468700 & -2.66265100 & $\mathrm{C}$ & -5.74310500 & 3.09941600 & -1.11696200 \\
\hline $\mathrm{H}$ & 2.93412000 & -4.94771100 & -2.74283900 & $\mathrm{C}$ & -4.45451200 & 3.09752200 & -1.65280500 \\
\hline $\mathrm{C}$ & 1.41812300 & -2.21103800 & 1.67998400 & $\mathrm{C}$ & -3.50531000 & 2.17933800 & -1.19719400 \\
\hline
\end{tabular}




\begin{tabular}{|c|c|c|c|c|c|c|c|}
\hline $\mathrm{C}$ & -3.83789300 & 1.25112900 & -0.20091300 & $\mathrm{H}$ & 0.58036400 & -3.89811400 & -2.63608200 \\
\hline $\mathrm{H}$ & 2.33977800 & 0.49984400 & 2.59723400 & $\mathrm{H}$ & 2.80639700 & -5.01870300 & -2.65489900 \\
\hline $\mathrm{H}$ & 2.73535400 & 2.22657000 & 2.47684700 & $\mathrm{C}$ & 1.25091600 & -2.16290300 & 1.68243200 \\
\hline $\mathrm{H}$ & 0.63692500 & 1.73666300 & 3.84542800 & $\mathrm{H}$ & 2.09120500 & -1.48532200 & 1.91527200 \\
\hline $\mathrm{H}$ & 0.34847000 & 2.83047100 & 2.48739300 & $\mathrm{O}$ & 0.07342200 & -1.41834900 & 1.45453000 \\
\hline $\mathrm{H}$ & -3.72491200 & 0.25722900 & 2.57850600 & $\mathrm{H}$ & -0.04697400 & -0.47962600 & 2.10921700 \\
\hline $\mathrm{H}$ & -2.41450900 & -0.93838600 & 2.57543900 & $\mathrm{C}$ & 1.02488600 & -3.07556800 & 2.89869800 \\
\hline $\mathrm{H}$ & -2.06753500 & 2.11426300 & 2.51821800 & $\mathrm{H}$ & 0.20102900 & -3.76696200 & 2.69038400 \\
\hline $\mathrm{H}$ & -1.68576600 & 1.03632900 & 3.86974900 & $\mathrm{H}$ & 1.91816100 & -3.66267900 & 3.13967800 \\
\hline $\mathrm{H}$ & 2.36700800 & -0.46818800 & -1.92652400 & $\mathrm{H}$ & 0.75727700 & -2.46991900 & 3.77240900 \\
\hline $\mathrm{H}$ & 4.28067300 & -1.84164500 & -2.64843500 & & & & \\
\hline $\mathrm{H}$ & 6.34143900 & -1.94696200 & -1.25818300 & \multicolumn{4}{|c|}{ ab6 (B3LYP-D3 optimized) } \\
\hline $\mathrm{H}$ & 6.46538900 & -0.66066000 & 0.86651200 & $\mathrm{C}$ & -4.35216400 & -1.57119600 & -1.27446400 \\
\hline $\mathrm{H}$ & 4.56555200 & 0.74449300 & 1.57803400 & $\mathrm{C}$ & -2.99201400 & -1.58738600 & -0.96555600 \\
\hline $\mathrm{H}$ & 0.34079200 & 3.72528800 & 0.13554800 & $\mathrm{C}$ & -2.48490100 & -0.86319400 & 0.12735200 \\
\hline $\mathrm{H}$ & 0.88135400 & 6.04860600 & -0.52388100 & $\mathrm{C}$ & -3.39887900 & -0.14498300 & 0.91673200 \\
\hline $\mathrm{H}$ & 3.15603300 & 6.60987600 & -1.36493200 & $\mathrm{C}$ & -4.75902400 & -0.12325700 & 0.60897200 \\
\hline $\mathrm{H}$ & 4.88787200 & 4.83043300 & -1.51347600 & $\mathrm{C}$ & -5.24658600 & -0.83422000 & -0.49129300 \\
\hline $\mathrm{H}$ & 4.36483300 & 2.52468700 & -0.81019900 & $\mathrm{H}$ & -4.71833600 & -2.14373400 & -2.12534400 \\
\hline $\mathrm{H}$ & -1.81208900 & -2.65560100 & 1.06190900 & $\mathrm{H}$ & -2.31404900 & -2.18527400 & -1.56798100 \\
\hline $\mathrm{H}$ & -2.61287400 & -4.90199700 & 0.39935900 & $\mathrm{H}$ & -3.00032100 & 0.38598800 & 1.77500500 \\
\hline $\mathrm{H}$ & -4.50369100 & -5.13194100 & -1.20518500 & $\mathrm{H}$ & -5.44463300 & 0.44945700 & 1.23189100 \\
\hline $\mathrm{H}$ & -5.59152300 & -3.09385000 & -2.12652300 & $\mathrm{H}$ & -6.30830900 & -0.82329000 & -0.73059900 \\
\hline $\mathrm{H}$ & -4.81969900 & -0.85363200 & -1.44042100 & $\mathrm{C}$ & -1.02960700 & -0.82894200 & 0.51776800 \\
\hline $\mathrm{H}$ & -5.42684000 & 0.52249200 & 1.07639900 & $\mathrm{O}$ & -0.77500800 & -0.41553300 & 1.72940900 \\
\hline $\mathrm{H}$ & -7.09029800 & 2.16655100 & 0.28912800 & $\mathrm{C}$ & -0.06342800 & -1.16111000 & -0.40308700 \\
\hline $\mathrm{H}$ & -6.47957500 & 3.81689600 & -1.46913400 & $\mathrm{H}$ & 0.78133200 & -0.15478900 & 1.51961700 \\
\hline $\mathrm{H}$ & -4.18358100 & 3.81189100 & -2.42557600 & $\mathrm{H}$ & -0.31935100 & -1.45449000 & -1.41633700 \\
\hline $\mathrm{H}$ & -2.49991500 & 2.18179100 & -1.60542400 & $\mathrm{H}$ & 1.79167100 & 1.50515700 & 0.34354900 \\
\hline $\mathrm{C}$ & -0.21527400 & 0.01186100 & -1.75109300 & $\mathrm{C}$ & 0.66458300 & 3.06164100 & -0.12552000 \\
\hline $\mathrm{O}$ & -0.16701000 & -0.27489000 & -2.87988900 & $\mathrm{C}$ & -0.14546200 & 2.45347600 & -1.28524600 \\
\hline $\mathrm{H}$ & -0.70139000 & 1.94811400 & -0.41124900 & $\mathrm{H}$ & -0.32880900 & 1.38954000 & -1.09909300 \\
\hline $\mathrm{C}$ & 3.32367200 & -4.33258800 & -0.67274200 & $\mathrm{H}$ & -1.11485100 & 2.95566000 & -1.40776000 \\
\hline $\mathrm{C}$ & 2.91379500 & -3.59249800 & 0.43454000 & $\mathrm{H}$ & 0.41849600 & 2.54941500 & -2.22165100 \\
\hline $\mathrm{C}$ & 1.66209700 & -2.96269700 & 0.45588400 & $\mathrm{C}$ & -0.11986800 & 2.93522000 & 1.19295200 \\
\hline $\mathrm{C}$ & 0.82707400 & -3.08947500 & -0.65842900 & $\mathrm{H}$ & -0.37143700 & 1.88935900 & 1.40381600 \\
\hline $\mathrm{C}$ & 1.23900300 & -3.82262500 & -1.77467100 & $\mathrm{H}$ & 0.48754900 & 3.32221000 & 2.02065800 \\
\hline $\mathrm{C}$ & 2.48629600 & -4.44849100 & -1.78639400 & $\mathrm{H}$ & -1.05648700 & 3.50799900 & 1.15242700 \\
\hline $\mathrm{H}$ & 4.30274900 & -4.80532300 & -0.67404800 & $\mathrm{C}$ & 0.98648800 & 4.53322600 & -0.41702300 \\
\hline $\mathrm{H}$ & 3.58484400 & -3.47993400 & 1.28350300 & $\mathrm{H}$ & 1.56619400 & 4.96227600 & 0.40881000 \\
\hline $\mathrm{H}$ & -0.13866800 & -2.60112700 & -0.65569000 & $\mathrm{H}$ & 1.58869500 & 4.61083300 & -1.33023400 \\
\hline
\end{tabular}




\begin{tabular}{|c|c|c|c|c|c|c|c|}
\hline $\mathrm{H}$ & 0.07053300 & 5.12274500 & -0.54908800 & $\mathrm{H}$ & -1.89340700 & 1.49390700 & 1.36422300 \\
\hline $\mathrm{O}$ & 1.93055600 & 2.41690100 & -0.01828000 & $\mathrm{H}$ & -2.86783500 & 2.91637500 & 1.85106300 \\
\hline $\mathrm{C}$ & 3.77817000 & -0.74772700 & -0.90583900 & $\mathrm{H}$ & -3.60492700 & 1.29294900 & 1.80088100 \\
\hline $\mathrm{H}$ & 3.96312500 & 0.04069100 & -0.16614700 & $\mathrm{C}$ & -2.23891700 & 3.07014300 & -0.85435300 \\
\hline $\mathrm{H}$ & 4.37131100 & -0.49661000 & -1.79706800 & $\mathrm{H}$ & -1.25209200 & 2.59933400 & -0.79153000 \\
\hline $\mathrm{C}$ & 2.28152000 & -0.74411900 & -1.24943200 & $\mathrm{H}$ & -2.49718700 & 3.19552400 & -1.91337400 \\
\hline $\mathrm{H}$ & 1.98082300 & 0.24826200 & -1.60061600 & $\mathrm{H}$ & -2.19909200 & 4.06248700 & -0.38240400 \\
\hline $\mathrm{H}$ & 2.08338200 & -1.46051500 & -2.06156600 & $\mathrm{C}$ & -4.68013000 & 2.70827100 & -0.30080600 \\
\hline $\mathrm{C}$ & 4.22342400 & -2.10207100 & -0.33187800 & $\mathrm{H}$ & -4.94736500 & 2.81498100 & -1.35917700 \\
\hline $\mathrm{H}$ & 5.28864000 & -2.07180700 & -0.05877400 & $\mathrm{H}$ & -5.40016200 & 2.02211600 & 0.16117100 \\
\hline $\mathrm{H}$ & 4.12022200 & -2.87608700 & -1.11025900 & $\mathrm{H}$ & -4.76708700 & 3.68963700 & 0.18508200 \\
\hline $\mathrm{C}$ & 3.36619000 & -2.49233200 & 0.88237700 & $\mathrm{O}$ & -3.27026000 & 0.88505900 & -0.83064200 \\
\hline $\mathrm{H}$ & 3.52409500 & -1.75977900 & 1.68337500 & $\mathrm{C}$ & -2.48946800 & -1.99758500 & 1.06146000 \\
\hline $\mathrm{H}$ & 3.67403000 & -3.47539800 & 1.26882800 & $\mathrm{H}$ & -2.79997100 & -1.03294400 & 0.65480500 \\
\hline $\mathrm{C}$ & 1.40451000 & -1.12396100 & -0.03933000 & $\mathrm{H}$ & -3.15296000 & -2.23604600 & 1.90641900 \\
\hline $\mathrm{C}$ & 1.87271000 & -2.49358200 & 0.52507300 & $\mathrm{C}$ & -1.04360400 & -1.89684200 & 1.57020900 \\
\hline $\mathrm{H}$ & 1.66496500 & -3.27456000 & -0.22251800 & $\mathrm{H}$ & -0.93409600 & -1.03436600 & 2.23574100 \\
\hline $\mathrm{H}$ & 1.25818700 & -2.71554900 & 1.40538900 & $\mathrm{H}$ & -0.82170900 & -2.79650700 & 2.17364400 \\
\hline $\mathrm{O}$ & 1.64812100 & -0.13144300 & 0.98341000 & $\mathrm{C}$ & -2.62495800 & -3.06328700 & -0.03457200 \\
\hline & & & & $\mathrm{H}$ & -3.65926300 & -3.09201800 & -0.40426400 \\
\hline & D3 optimi & ed) & & $\mathrm{H}$ & -2.40852300 & -4.06162200 & 0.38339400 \\
\hline & dency $=-74$ & $94 \mathrm{~cm}^{-1}$ & & $\mathrm{C}$ & -1.65541700 & -2.77298700 & -1.18728300 \\
\hline $\mathrm{C}$ & 3.32260800 & 2.44715500 & 0.32254600 & $\mathrm{H}$ & -1.89769100 & -1.79534600 & -1.61401900 \\
\hline $\mathrm{C}$ & 2.57228500 & 1.26959200 & 0.27857300 & $\mathrm{H}$ & -1.75764200 & -3.53250300 & -1.97634800 \\
\hline $\mathrm{C}$ & 3.20814500 & 0.03969400 & 0.04506500 & $\mathrm{C}$ & 0.02417900 & -1.83882500 & 0.49439800 \\
\hline $\mathrm{C}$ & 4.59121300 & 0.01075500 & -0.17204200 & $\mathrm{C}$ & -0.19863300 & -2.73620700 & -0.70274300 \\
\hline $\mathrm{C}$ & 5.34208300 & 1.18401700 & -0.10673400 & $\mathrm{H}$ & 0.10485300 & -3.75294400 & -0.39795200 \\
\hline $\mathrm{C}$ & 4.70832900 & 2.40617600 & 0.14460200 & $\mathrm{H}$ & 0.48633600 & -2.46322500 & -1.50876900 \\
\hline $\mathrm{H}$ & 2.82301100 & 3.39929500 & 0.48805400 & $\mathrm{O}$ & -0.89796000 & 0.08713300 & -0.37361400 \\
\hline $\mathrm{H}$ & 1.48687300 & 1.28500900 & 0.37017300 & & & & \\
\hline $\mathrm{H}$ & 5.05559300 & -0.94549600 & -0.39750300 & \multicolumn{2}{|c|}{ 6a ( $\omega$ B97XD optimized) } & & \\
\hline $\mathrm{H}$ & 6.41943400 & 1.15163200 & -0.25858400 & $\mathrm{Ru}$ & 0.31594900 & 0.42614100 & -0.04777800 \\
\hline $\mathrm{H}$ & 5.29198200 & 3.32371200 & 0.18784300 & $\mathrm{P}$ & 2.60849200 & 0.14994800 & 0.31282500 \\
\hline $\mathrm{C}$ & 2.39031800 & -1.23011500 & -0.07708100 & $\mathrm{P}$ & -1.95111400 & 0.92113000 & 0.28417100 \\
\hline $\mathrm{O}$ & 2.72964500 & -2.06622000 & -0.93414300 & $\mathrm{~N}$ & 0.29046400 & 0.36253400 & 2.05576200 \\
\hline $\mathrm{C}$ & 1.25382800 & -1.31270200 & 0.80776500 & $\mathrm{C}$ & 2.69888100 & -0.03788800 & 2.14671600 \\
\hline $\mathrm{H}$ & -0.30588300 & 0.11402700 & -1.14228000 & $\mathrm{C}$ & 1.52336300 & 0.73437300 & 2.74313400 \\
\hline $\mathrm{H}$ & 1.28819500 & -0.66220100 & 1.67910600 & $\mathrm{C}$ & -2.15141100 & 0.48290600 & 2.06120700 \\
\hline $\mathrm{H}$ & -2.30139800 & 0.51287800 & -0.73911300 & $\mathrm{C}$ & -0.86242500 & 0.91798500 & 2.75373000 \\
\hline $\mathrm{C}$ & -3.26210200 & 2.13503700 & -0.17495500 & $\mathrm{C}$ & 4.40371900 & -2.04425200 & 0.39478800 \\
\hline $\mathrm{C}$ & -2.88299000 & 1.95830700 & 1.31110000 & $\mathrm{C}$ & 5.00151300 & -3.18529300 & -0.13218000 \\
\hline
\end{tabular}




\begin{tabular}{|c|c|c|c|c|c|c|c|}
\hline $\mathrm{C}$ & 4.66372500 & -3.62286200 & -1.41009000 & $\mathrm{H}$ & -6.41136700 & -1.24907700 & -0.15205400 \\
\hline $\mathrm{C}$ & 3.72984700 & -2.91387500 & -2.16060500 & $\mathrm{H}$ & -6.30615500 & -1.33843300 & -2.62956800 \\
\hline $\mathrm{C}$ & 3.12827200 & -1.77633000 & -1.63189400 & $\mathrm{H}$ & -4.27523400 & -0.52679300 & -3.80774500 \\
\hline $\mathrm{C}$ & 3.45882700 & -1.33007200 & -0.34908400 & $\mathrm{H}$ & -2.38136500 & 0.38953000 & -2.52111900 \\
\hline $\mathrm{C}$ & 3.33315100 & 2.58167300 & -0.89204900 & $\mathrm{H}$ & -4.38866700 & 2.32019900 & 1.13385700 \\
\hline $\mathrm{C}$ & 4.21216300 & 3.61125000 & -1.22233300 & $\mathrm{H}$ & -5.10489400 & 4.67272400 & 1.05081700 \\
\hline $\mathrm{C}$ & 5.52598900 & 3.58040200 & -0.76747900 & $\mathrm{H}$ & -3.62651400 & 6.39211600 & 0.03720100 \\
\hline $\mathrm{C}$ & 5.96532500 & 2.51271800 & 0.01402200 & $\mathrm{H}$ & -1.42051000 & 5.72900800 & -0.88718600 \\
\hline $\mathrm{C}$ & 5.08970600 & 1.48412800 & 0.33925300 & $\mathrm{H}$ & -0.68896700 & 3.36785200 & -0.77618500 \\
\hline $\mathrm{C}$ & 3.76217000 & 1.51223000 & -0.10436700 & $\mathrm{C}$ & 0.31388500 & 0.38601100 & -1.91716200 \\
\hline $\mathrm{C}$ & -4.46755900 & -0.35877300 & 0.05298900 & $\mathrm{O}$ & 0.31321800 & 0.39559600 & -3.07961200 \\
\hline $\mathrm{C}$ & -5.53464800 & -0.87815300 & -0.67463700 & $\mathrm{H}$ & 0.58180800 & 1.95904400 & -0.11247000 \\
\hline $\mathrm{C}$ & -5.47265500 & -0.93352700 & -2.06299400 & $\mathrm{C}$ & -3.16047200 & -3.44475200 & -0.99843900 \\
\hline $\mathrm{C}$ & -4.33412100 & -0.47756300 & -2.72449000 & $\mathrm{C}$ & -1.85397300 & -3.04518900 & -0.74498500 \\
\hline $\mathrm{C}$ & -3.26774700 & 0.04097800 & -1.99896200 & $\mathrm{C}$ & -1.35930800 & -3.01486200 & 0.56063400 \\
\hline $\mathrm{C}$ & -3.32986400 & 0.11427900 & -0.60356100 & $\mathrm{C}$ & -2.20350000 & -3.36434700 & 1.61294900 \\
\hline $\mathrm{C}$ & -3.72177900 & 3.06608300 & 0.70909400 & $\mathrm{C}$ & -3.51796800 & -3.75486700 & 1.36238100 \\
\hline $\mathrm{C}$ & -4.13396500 & 4.39165400 & 0.65370900 & $\mathrm{C}$ & -3.99606000 & -3.80550100 & 0.05664300 \\
\hline $\mathrm{C}$ & -3.30470000 & 5.35581400 & 0.08276300 & $\mathrm{H}$ & -3.53653400 & -3.45006700 & -2.01690500 \\
\hline $\mathrm{C}$ & -2.06846300 & 4.98459300 & -0.43399100 & $\mathrm{H}$ & -1.20873100 & -2.74532500 & -1.56886200 \\
\hline $\mathrm{C}$ & -1.65385900 & 3.65542800 & -0.37237500 & $\mathrm{H}$ & -1.81072200 & -3.32985400 & 2.62430100 \\
\hline $\mathrm{C}$ & -2.47167600 & 2.68426700 & 0.20642100 & $\mathrm{H}$ & -4.16695400 & -4.02695000 & 2.19065300 \\
\hline $\mathrm{H}$ & 2.55900200 & -1.10361900 & 2.35743000 & $\mathrm{H}$ & -5.02074200 & -4.10738000 & -0.14043200 \\
\hline $\mathrm{H}$ & 3.65819300 & 0.29221800 & 2.55755900 & $\mathrm{C}$ & 0.10101100 & -2.67545200 & 0.82231000 \\
\hline $\mathrm{H}$ & 1.45144400 & 0.48773700 & 3.81568200 & $\mathrm{H}$ & 0.36706600 & -1.82123900 & 0.14218000 \\
\hline $\mathrm{H}$ & 1.71292500 & 1.82065200 & 2.68158300 & $\mathrm{O}$ & 0.35617100 & -2.33900800 & 2.14973400 \\
\hline $\mathrm{H}$ & -3.03151400 & 0.94666700 & 2.51805900 & $\mathrm{H}$ & 0.23835800 & -1.34069800 & 2.21992600 \\
\hline $\mathrm{H}$ & -2.25358400 & -0.60753300 & 2.11515400 & $\mathrm{C}$ & 1.00247600 & -3.84349300 & 0.42376400 \\
\hline $\mathrm{H}$ & -0.82409300 & 2.02224200 & 2.79467500 & $\mathrm{H}$ & 0.74835600 & -4.71742200 & 1.03297700 \\
\hline $\mathrm{H}$ & -0.88079600 & 0.56669200 & 3.79896300 & $\mathrm{H}$ & 0.87215500 & -4.10317400 & -0.63134400 \\
\hline $\mathrm{H}$ & 4.66460900 & -1.72601500 & 1.40016400 & $\mathrm{H}$ & 2.05025400 & -3.58505900 & 0.59797100 \\
\hline
\end{tabular}

$\begin{array}{crrr}\text { TS3a }(\omega B 97 X D \text { optimized }) & \\ \text { imaginary frequency }=-688.03 \mathrm{~cm}^{-1} & \\ \mathrm{Ru} & 0.34704400 & 0.37817500 & -0.06299000 \\ \mathrm{P} & 2.63131500 & 0.01542300 & 0.29050700 \\ \mathrm{P} & -1.89581300 & 0.97507700 & 0.26347000 \\ \mathrm{~N} & 0.32447300 & 0.34115000 & 2.08687900 \\ \mathrm{C} & 2.69246100 & -0.22396800 & 2.12090700 \\ \mathrm{C} & 1.60363300 & 0.65122400 & 2.73622100 \\ \mathrm{C} & -2.09880500 & 0.61843600 & 2.06049200\end{array}$




\begin{tabular}{|c|c|c|c|c|c|c|c|}
\hline $\mathrm{C}$ & -0.79521700 & 1.02921700 & 2.73981900 & $\mathrm{H}$ & 7.06362000 & 2.24099700 & 0.50174100 \\
\hline $\mathrm{C}$ & 4.39536200 & -2.20209700 & 0.32661500 & $\mathrm{H}$ & 5.47225600 & 0.42041400 & 0.96305900 \\
\hline $\mathrm{C}$ & 4.99120200 & -3.32781900 & -0.23359600 & $\mathrm{H}$ & -4.43112200 & -0.32670700 & 1.18369100 \\
\hline $\mathrm{C}$ & 4.67460700 & -3.70983100 & -1.53443400 & $\mathrm{H}$ & -6.34790600 & -1.22949300 & -0.06315300 \\
\hline $\mathrm{C}$ & 3.76167700 & -2.96268000 & -2.27374900 & $\mathrm{H}$ & -6.32227800 & -1.26772500 & -2.54359100 \\
\hline $\mathrm{C}$ & 3.16024800 & -1.84138200 & -1.71164900 & $\mathrm{H}$ & -4.33630400 & -0.41775400 & -3.77005900 \\
\hline $\mathrm{C}$ & 3.47246300 & -1.44996000 & -0.40696200 & $\mathrm{H}$ & -2.41079300 & 0.49244100 & -2.52660000 \\
\hline $\mathrm{C}$ & 3.42566900 & 2.48465800 & -0.79665100 & $\mathrm{H}$ & -4.34472400 & 2.43449500 & 0.93194900 \\
\hline $\mathrm{C}$ & 4.33264900 & 3.50691800 & -1.06913000 & $\mathrm{H}$ & -4.99785600 & 4.80448300 & 0.78936900 \\
\hline $\mathrm{C}$ & 5.63888300 & 3.42532000 & -0.59910400 & $\mathrm{H}$ & -3.41812000 & 6.47960400 & -0.14196900 \\
\hline $\mathrm{C}$ & 6.04279700 & 2.31341500 & 0.13855100 & $\mathrm{H}$ & -1.17552700 & 5.75743800 & -0.92208100 \\
\hline $\mathrm{C}$ & 5.13958500 & 1.29178200 & 0.40494300 & $\mathrm{H}$ & -0.50920100 & 3.37886000 & -0.74909900 \\
\hline $\mathrm{C}$ & 3.81874800 & 1.37102500 & -0.05277600 & $\mathrm{C}$ & 0.34577100 & 0.38108300 & -1.92629600 \\
\hline $\mathrm{C}$ & -4.40945700 & -0.32119200 & 0.09846500 & $\mathrm{O}$ & 0.34682800 & 0.41227100 & -3.08648300 \\
\hline $\mathrm{C}$ & -5.49383900 & -0.83630700 & -0.60607600 & $\mathrm{H}$ & 0.68269500 & 1.91499800 & -0.06953600 \\
\hline $\mathrm{C}$ & -5.47541200 & -0.86433700 & -1.99618000 & $\mathrm{C}$ & -3.30230800 & -3.49046000 & -0.99135200 \\
\hline $\mathrm{C}$ & -4.36188000 & -0.38757100 & -2.68486200 & $\mathrm{C}$ & -2.03201600 & -3.01201500 & -0.69460400 \\
\hline $\mathrm{C}$ & -3.27783400 & 0.12643700 & -1.98367900 & $\mathrm{C}$ & -1.60936200 & -2.88665300 & 0.63049100 \\
\hline $\mathrm{C}$ & -3.29826400 & 0.17358400 & -0.58570900 & $\mathrm{C}$ & -2.49114300 & -3.22180400 & 1.65549200 \\
\hline $\mathrm{C}$ & -3.63575600 & 3.16258400 & 0.54566400 & $\mathrm{C}$ & -3.76887100 & -3.69640300 & 1.36215900 \\
\hline $\mathrm{C}$ & -4.01109100 & 4.49706200 & 0.45601500 & $\mathrm{C}$ & -4.17382300 & -3.84116300 & 0.03895000 \\
\hline $\mathrm{C}$ & -3.12457700 & 5.43652300 & -0.06882500 & $\mathrm{H}$ & -3.62236800 & -3.57153600 & -2.02605300 \\
\hline $\mathrm{C}$ & -1.86782300 & 5.03220100 & -0.50477000 & $\mathrm{H}$ & -1.35944700 & -2.72130700 & -1.49979400 \\
\hline $\mathrm{C}$ & -1.48997900 & 3.69393700 & -0.40903300 & $\mathrm{H}$ & -2.14441200 & -3.11219700 & 2.67849600 \\
\hline $\mathrm{C}$ & -2.36611600 & 2.74780100 & 0.12339100 & $\mathrm{H}$ & -4.44601500 & -3.96133200 & 2.17034600 \\
\hline $\mathrm{H}$ & 2.43378200 & -1.27180300 & 2.31349100 & $\mathrm{H}$ & -5.16915000 & -4.21047800 & -0.19254100 \\
\hline $\mathrm{H}$ & 3.67625400 & -0.00394700 & 2.54664600 & $\mathrm{C}$ & -0.18121000 & -2.46549000 & 0.97876400 \\
\hline $\mathrm{H}$ & 1.53841300 & 0.44196300 & 3.81462700 & $\mathrm{H}$ & 0.03703500 & -1.57667200 & 0.23928200 \\
\hline $\mathrm{H}$ & 1.84262700 & 1.71719200 & 2.61729700 & $\mathrm{O}$ & -0.00736400 & -2.10516000 & 2.26363700 \\
\hline $\mathrm{H}$ & -2.95976200 & 1.13346100 & 2.49787200 & $\mathrm{H}$ & 0.14063200 & -0.83938000 & 2.24970800 \\
\hline $\mathrm{H}$ & -2.23106600 & -0.46451700 & 2.16825800 & $\mathrm{C}$ & 0.80538800 & -3.55716900 & 0.53431700 \\
\hline $\mathrm{H}$ & -0.65817300 & 2.11863200 & 2.68423600 & $\mathrm{H}$ & 0.57714400 & -4.47743700 & 1.08274500 \\
\hline $\mathrm{H}$ & -0.83411500 & 0.75250600 & 3.80403000 & $\mathrm{H}$ & 0.74517900 & -3.76237500 & -0.54009200 \\
\hline $\mathrm{H}$ & 4.63842600 & -1.92781400 & 1.34930800 & $\mathrm{H}$ & 1.82721400 & -3.25509000 & 0.77622800 \\
\hline $\mathrm{H}$ & 5.69720000 & -3.91111900 & 0.34987900 & & & & \\
\hline $\mathrm{H}$ & 5.13450100 & -4.59234500 & -1.96888100 & \multicolumn{2}{|c|}{ 6b ( $\omega$ B97XD optimized) } & & \\
\hline $\mathrm{H}$ & 3.50651700 & -3.25942500 & -3.28648900 & $\mathrm{Ru}$ & -0.32447400 & 0.45963700 & 0.09134900 \\
\hline $\mathrm{H}$ & 2.43231100 & -1.27716100 & -2.28636400 & $\mathrm{P}$ & 1.83108000 & 1.30307800 & 0.37265200 \\
\hline $\mathrm{H}$ & 2.40541100 & 2.54794100 & -1.16069200 & $\mathrm{P}$ & -2.58750800 & -0.00787500 & 0.39677600 \\
\hline $\mathrm{H}$ & 4.01451300 & 4.36737100 & -1.65020100 & $\mathrm{~N}$ & -0.42042100 & 0.85507900 & 2.20443800 \\
\hline $\mathrm{H}$ & 6.34495500 & 4.22325400 & -0.80943500 & $\mathrm{C}$ & 1.93537900 & 1.51648600 & 2.21368400 \\
\hline
\end{tabular}




\begin{tabular}{|c|c|c|c|c|c|c|c|}
\hline $\mathrm{C}$ & 0.52805600 & 1.82575300 & 2.72315600 & $\mathrm{H}$ & 0.75010000 & 6.02240600 & -0.60543700 \\
\hline $\mathrm{C}$ & -2.72450800 & 0.03727700 & 2.24203100 & $\mathrm{H}$ & 3.02943700 & 6.61031800 & -1.39951100 \\
\hline $\mathrm{C}$ & -1.74795100 & 1.09849700 & 2.74725100 & $\mathrm{H}$ & 4.79508300 & 4.86660000 & -1.46944500 \\
\hline $\mathrm{C}$ & 3.27984200 & -0.35792700 & -1.31824500 & $\mathrm{H}$ & 4.29878800 & 2.56784300 & -0.73782600 \\
\hline $\mathrm{C}$ & 4.38253800 & -1.09636500 & -1.73239700 & $\mathrm{H}$ & -1.76347600 & -2.68248200 & 1.03980400 \\
\hline $\mathrm{C}$ & 5.50459000 & -1.19348700 & -0.91441800 & $\mathrm{H}$ & -2.55509200 & -4.92937100 & 0.38598900 \\
\hline $\mathrm{C}$ & 5.52356100 & -0.54625100 & 0.31801700 & $\mathrm{H}$ & -4.48351600 & -5.17072100 & -1.16347000 \\
\hline $\mathrm{C}$ & 4.42600600 & 0.20721600 & 0.72661200 & $\mathrm{H}$ & -5.61647700 & -3.14432700 & -2.04085100 \\
\hline $\mathrm{C}$ & 3.29439800 & 0.30960700 & -0.08907700 & $\mathrm{H}$ & -4.85071300 & -0.90283700 & -1.36734800 \\
\hline $\mathrm{C}$ & 1.24351800 & 3.96669900 & -0.23166900 & $\mathrm{H}$ & -5.40991700 & 0.52111500 & 1.11394400 \\
\hline $\mathrm{C}$ & 1.53045900 & 5.26747600 & -0.63209300 & $\mathrm{H}$ & -7.07108200 & 2.13449200 & 0.27618800 \\
\hline $\mathrm{C}$ & 2.80823500 & 5.59685800 & -1.07755000 & $\mathrm{H}$ & -6.47163000 & 3.70595300 & -1.55028000 \\
\hline $\mathrm{C}$ & 3.79825200 & 4.61974700 & -1.11580400 & $\mathrm{H}$ & -4.18876400 & 3.65346300 & -2.52363900 \\
\hline $\mathrm{C}$ & 3.51667900 & 3.31989400 & -0.70307600 & $\mathrm{H}$ & -2.50628500 & 2.05650800 & -1.65335300 \\
\hline $\mathrm{C}$ & 2.23619400 & 2.98050000 & -0.25609600 & $\mathrm{C}$ & -0.23530600 & 0.01798300 & -1.72007300 \\
\hline $\mathrm{C}$ & -2.62968700 & -2.78528200 & 0.39129300 & $\mathrm{O}$ & -0.19787600 & -0.22972000 & -2.85339200 \\
\hline $\mathrm{C}$ & -3.06689200 & -4.04842300 & 0.00956500 & $\mathrm{H}$ & -0.75409500 & 1.90749200 & -0.34977400 \\
\hline $\mathrm{C}$ & -4.14547300 & -4.18343500 & -0.86236100 & $\mathrm{C}$ & 3.46695600 & -4.22029900 & -0.78270400 \\
\hline $\mathrm{C}$ & -4.78085300 & -3.04771800 & -1.35376000 & $\mathrm{C}$ & 3.04826700 & -3.52668400 & 0.34605600 \\
\hline $\mathrm{C}$ & -4.34813600 & -1.77962800 & -0.97133700 & $\mathrm{C}$ & 1.77848100 & -2.94758400 & 0.39822100 \\
\hline $\mathrm{C}$ & -3.27373800 & -1.63719300 & -0.08983500 & $\mathrm{C}$ & 0.93332700 & -3.07389900 & -0.70288100 \\
\hline $\mathrm{C}$ & -5.12801900 & 1.21955200 & 0.33056800 & $\mathrm{C}$ & 1.35570500 & -3.75924700 & -1.84025800 \\
\hline $\mathrm{C}$ & -6.07183800 & 2.12084800 & -0.14887200 & $\mathrm{C}$ & 2.62076300 & -4.33661500 & -1.88404700 \\
\hline $\mathrm{C}$ & -5.73494600 & 3.00140200 & -1.17535400 & $\mathrm{H}$ & 4.46159700 & -4.65654700 & -0.80964200 \\
\hline $\mathrm{C}$ & -4.45521800 & 2.97313400 & -1.72032200 & $\mathrm{H}$ & 3.72807700 & -3.40998200 & 1.18726100 \\
\hline $\mathrm{C}$ & -3.50799900 & 2.07370700 & -1.23596400 & $\mathrm{H}$ & -0.05170400 & -2.62274100 & -0.67593700 \\
\hline $\mathrm{C}$ & -3.83342700 & 1.19148900 & -0.20312200 & $\mathrm{H}$ & 0.68887900 & -3.83669000 & -2.69407100 \\
\hline $\mathrm{H}$ & 2.27254300 & 0.56539100 & 2.64156300 & $\mathrm{H}$ & 2.94822400 & -4.87176800 & -2.77093800 \\
\hline $\mathrm{H}$ & 2.65285400 & 2.29541300 & 2.49113300 & $\mathrm{C}$ & 1.35890300 & -2.19943600 & 1.64612100 \\
\hline $\mathrm{H}$ & 0.54101600 & 1.80850900 & 3.82719900 & $\mathrm{H}$ & 2.15052300 & -1.46747200 & 1.87002000 \\
\hline $\mathrm{H}$ & 0.25636600 & 2.85918600 & 2.43666900 & $\mathrm{O}$ & 0.13771500 & -1.52345400 & 1.42595500 \\
\hline $\mathrm{H}$ & -3.74709600 & 0.22212300 & 2.58440300 & $\mathrm{H}$ & 0.01094600 & -0.74826300 & 2.08295100 \\
\hline $\mathrm{H}$ & -2.41765700 & -0.94969300 & 2.60553500 & $\mathrm{C}$ & 1.20230600 & -3.13451400 & 2.84691200 \\
\hline $\mathrm{H}$ & -2.12595800 & 2.10106000 & 2.47032100 & $\mathrm{H}$ & 0.42062200 & -3.87274900 & 2.64022400 \\
\hline $\mathrm{H}$ & -1.72932800 & 1.06805400 & 3.85079000 & $\mathrm{H}$ & 2.13386900 & -3.66735900 & 3.06335800 \\
\hline $\mathrm{H}$ & 2.39821400 & -0.31277800 & -1.94913500 & $\mathrm{H}$ & 0.91624200 & -2.56083100 & 3.73543700 \\
\hline $\mathrm{H}$ & 4.35077400 & -1.61937000 & -2.68251300 & & & & \\
\hline $\mathrm{H}$ & 6.36028900 & -1.78201600 & -1.23228500 & \multicolumn{4}{|c|}{ TS3b ( $\omega$ B97XD optimized) } \\
\hline $\mathrm{H}$ & 6.39577400 & -0.62121600 & 0.96102200 & \multicolumn{4}{|c|}{ imaginary frequency $=-868.03 \mathrm{~cm}^{-1}$} \\
\hline $\mathrm{H}$ & 4.46039800 & 0.72380300 & 1.68173900 & $\mathrm{Ru}$ & -0.31263200 & 0.47553000 & 0.06055800 \\
\hline $\mathrm{H}$ & 0.23912800 & 3.70644800 & 0.08855700 & $\mathrm{P}$ & 1.86507900 & 1.26410600 & 0.34794700 \\
\hline
\end{tabular}

$\mathrm{H}$

$\mathrm{H}$

$\mathrm{H}$

$\mathrm{H}$

$\mathrm{H}$

$$
\begin{array}{rrr}
0.75010000 & 6.02240600 & -0.60543700 \\
3.02943700 & 6.61031800 & -1.39951100 \\
4.79508300 & 4.86660000 & -1.46944500 \\
4.29878800 & 2.56784300 & -0.73782600 \\
-1.76347600 & -2.68248200 & 1.03980400 \\
-2.55509200 & -4.92937100 & 0.38598900 \\
-4.48351600 & -5.17072100 & -1.16347000 \\
-5.61647700 & -3.14432700 & -2.04085100 \\
-4.85071300 & -0.90283700 & -1.36734800 \\
-5.40991700 & 0.52111500 & 1.11394400 \\
-7.07108200 & 2.13449200 & 0.27618800 \\
-6.47163000 & 3.70595300 & -1.55028000 \\
-4.18876400 & 3.65346300 & -2.52363900 \\
-2.50628500 & 2.05650800 & -1.65335300 \\
-0.23530600 & 0.01798300 & -1.72007300 \\
-0.19787600 & -0.22972000 & -2.85339200 \\
-0.75409500 & 1.90749200 & -0.34977400 \\
\hline 3.46695600 & -4.22029900 & -0.78270400 \\
\hline 3.04826700 & -3.52668400 & 0.34605600 \\
\hline .1 .77848100 & -2.94758400 & 0.39822100 \\
0.93332700 & -3.07389900 & -0.70288100 \\
1.35570500 & -3.75924700 & -1.84025800 \\
2.62076300 & -4.33661500 & -1.88404700 \\
4.46159700 & -4.65654700 & -0.80964200 \\
3.72807700 & -3.40998200 & 1.18726100 \\
-0.05170400 & -2.62274100 & -0.67593700 \\
0.68887900 & -3.83669000 & -2.69407100 \\
\hline .94822400 & -4.87176800 & -2.77093800 \\
\hline .35890300 & -2.19943600 & 1.64612100 \\
\hline .15052300 & -1.46747200 & 1.87002000 \\
\hline & -2.56083100 & 3.73543700 \\
\hline
\end{array}
$$

TS3b ( $\omega$ B97XD optimized) imaginary frequency $=-868.03 \mathrm{~cm}^{-1}$

$\begin{array}{lrrr}\mathrm{Ru} & -0.31263200 & 0.47553000 & 0.06055800 \\ \mathrm{P} & 1.86507900 & 1.26410600 & 0.34794700\end{array}$




\begin{tabular}{|c|c|c|c|c|c|c|c|}
\hline$P$ & -2.58067100 & 0.02517800 & 0.37187300 & $\mathrm{H}$ & 6.39425600 & -0.74494700 & 0.91418100 \\
\hline $\mathrm{N}$ & -0.39407100 & 0.84277800 & 2.19639000 & $\mathrm{H}$ & 4.49205900 & 0.64204500 & 1.64134300 \\
\hline $\mathrm{C}$ & 1.97910100 & 1.45702200 & 2.19356000 & $\mathrm{H}$ & 0.33237900 & 3.70695300 & 0.09521000 \\
\hline $\mathrm{C}$ & 0.58499500 & 1.77866100 & 2.72980200 & $\mathrm{H}$ & 0.89994800 & 6.02094200 & -0.56253800 \\
\hline $\mathrm{C}$ & -2.70473500 & 0.04007800 & 2.22061000 & $\mathrm{H}$ & 3.19281600 & 6.56471700 & -1.34876600 \\
\hline $\mathrm{C}$ & -1.72062400 & 1.08087500 & 2.75255300 & $\mathrm{H}$ & 4.91495300 & 4.77948700 & -1.44705700 \\
\hline $\mathrm{C}$ & 3.26474700 & -0.43762400 & -1.34089000 & $\mathrm{H}$ & 4.36310500 & 2.48271300 & -0.74928500 \\
\hline $\mathrm{C}$ & 4.34906900 & -1.20091200 & -1.75834800 & $\mathrm{H}$ & -1.77619800 & -2.64689600 & 1.01692400 \\
\hline $\mathrm{C}$ & 5.47596700 & -1.31338700 & -0.94932400 & $\mathrm{H}$ & -2.57370200 & -4.89283100 & 0.35065100 \\
\hline $\mathrm{C}$ & 5.51845500 & -0.65721400 & 0.27775700 & $\mathrm{H}$ & -4.48928400 & -5.12050400 & -1.21701900 \\
\hline $\mathrm{C}$ & 4.43930300 & 0.11996500 & 0.69006800 & $\mathrm{H}$ & -5.60313600 & -3.08691300 & -2.10148200 \\
\hline $\mathrm{C}$ & 3.30313300 & 0.23801200 & -0.11702100 & $\mathrm{H}$ & -4.83232700 & -0.85037500 & -1.41595400 \\
\hline $\mathrm{C}$ & 1.34330800 & 3.94841800 & -0.21938500 & $\mathrm{H}$ & -5.41031900 & 0.50652300 & 1.08485100 \\
\hline $\mathrm{C}$ & 1.66167700 & 5.24768200 & -0.60043200 & $\mathrm{H}$ & -7.07609500 & 2.14142100 & 0.29792700 \\
\hline $\mathrm{C}$ & 2.94700700 & 5.55229600 & -1.04180800 & $\mathrm{H}$ & -6.47418700 & 3.78430900 & -1.46372400 \\
\hline $\mathrm{C}$ & 3.91271200 & 4.55186500 & -1.09588000 & $\mathrm{H}$ & -4.18534000 & 3.78179700 & -2.42366400 \\
\hline $\mathrm{C}$ & 3.59981600 & 3.25317400 & -0.70237000 & $\mathrm{H}$ & -2.49833100 & 2.16149900 & -1.60328000 \\
\hline $\mathrm{C}$ & 2.31173600 & 2.93893200 & -0.25900800 & $\mathrm{C}$ & -0.23329500 & 0.04038000 & -1.74749300 \\
\hline $\mathrm{C}$ & -2.63833600 & -2.74886100 & 0.36208400 & $\mathrm{O}$ & -0.20002400 & -0.20902400 & -2.87997500 \\
\hline $\mathrm{C}$ & -3.07826000 & -4.00855600 & -0.02761800 & $\mathrm{H}$ & -0.71126800 & 1.93629500 & -0.39116700 \\
\hline $\mathrm{C}$ & -4.14909400 & -4.13568500 & -0.91025100 & $\mathrm{C}$ & 3.37169400 & -4.24343700 & -0.67654600 \\
\hline $\mathrm{C}$ & -4.77401200 & -2.99581000 & -1.40583000 & $\mathrm{C}$ & 2.94156500 & -3.52339800 & 0.43128900 \\
\hline $\mathrm{C}$ & -4.33857200 & -1.73077100 & -1.01665200 & $\mathrm{C}$ & 1.68168300 & -2.92006100 & 0.44867700 \\
\hline $\mathrm{C}$ & -3.27255300 & -1.59733900 & -0.12371800 & $\mathrm{C}$ & 0.86050000 & -3.04870300 & -0.66989500 \\
\hline $\mathrm{C}$ & -5.12795500 & 1.23645700 & 0.33073600 & $\mathrm{C}$ & 1.29336600 & -3.76027300 & -1.78725800 \\
\hline $\mathrm{C}$ & -6.07397600 & 2.14972900 & -0.12040900 & $\mathrm{C}$ & 2.54750000 & -4.36275700 & -1.79432900 \\
\hline $\mathrm{C}$ & -5.73563100 & 3.07043700 & -1.11078000 & $\mathrm{H}$ & 4.35852100 & -4.69822400 & -0.67485400 \\
\hline $\mathrm{C}$ & -4.45252800 & 3.07012600 & -1.64824500 & $\mathrm{H}$ & 3.60444400 & -3.40673300 & 1.28620700 \\
\hline $\mathrm{C}$ & -3.50293400 & 2.15815500 & -1.19239300 & $\mathrm{H}$ & -0.11551300 & -2.57778200 & -0.66931200 \\
\hline $\mathrm{C}$ & -3.82988800 & 1.23602000 & -0.19580200 & $\mathrm{H}$ & 0.64340300 & -3.83970300 & -2.65405200 \\
\hline $\mathrm{H}$ & 2.31500300 & 0.50031200 & 2.60800400 & $\mathrm{H}$ & 2.88360500 & -4.91905700 & -2.66497600 \\
\hline $\mathrm{H}$ & 2.70535100 & 2.22682200 & 2.47290800 & $\mathrm{C}$ & 1.24144400 & -2.15036000 & 1.68060500 \\
\hline $\mathrm{H}$ & 0.60759700 & 1.73413400 & 3.83181100 & $\mathrm{H}$ & 2.06995600 & -1.46684200 & 1.93736800 \\
\hline $\mathrm{H}$ & 0.31827300 & 2.81542500 & 2.46395600 & $\mathrm{O}$ & 0.06671500 & -1.42276600 & 1.44829600 \\
\hline $\mathrm{H}$ & -3.72388400 & 0.22932900 & 2.57015700 & $\mathrm{H}$ & -0.05905200 & -0.48886600 & 2.10031200 \\
\hline $\mathrm{H}$ & -2.40625100 & -0.95641000 & 2.56253200 & $\mathrm{C}$ & 1.01666400 & -3.09001800 & 2.87030000 \\
\hline $\mathrm{H}$ & -2.07703100 & 2.09292700 & 2.49522500 & $\mathrm{H}$ & 0.21169800 & -3.79458300 & 2.63517800 \\
\hline $\mathrm{H}$ & -1.69591000 & 1.02288200 & 3.85375000 & $\mathrm{H}$ & 1.91820800 & -3.66298200 & 3.11215800 \\
\hline $\mathrm{H}$ & 2.37862100 & -0.38105900 & -1.96423600 & $\mathrm{H}$ & 0.72455600 & -2.50966600 & 3.75249400 \\
\hline $\mathrm{H}$ & 4.29847100 & -1.73113300 & -2.70353900 & & & & \\
\hline $\mathrm{H}$ & 6.31697800 & -1.92142900 & -1.26956100 & $\mathbf{a b}$ & optimized) & & \\
\hline
\end{tabular}




\begin{tabular}{|c|c|c|c|c|c|c|c|}
\hline $\mathrm{C}$ & 4.28059300 & -0.73864500 & 1.56277200 & $\mathrm{H}$ & -3.51111000 & -1.88998200 & -1.59375500 \\
\hline $\mathrm{C}$ & 2.92121200 & -0.72945500 & 1.26382600 & $\mathrm{H}$ & -3.63065000 & -3.57324800 & -1.05938100 \\
\hline $\mathrm{C}$ & 2.47191500 & -0.82587400 & -0.05860100 & $\mathrm{C}$ & -1.40995800 & -1.10776800 & 0.08784800 \\
\hline $\mathrm{C}$ & 3.43188000 & -0.90181900 & -1.07328600 & $\mathrm{C}$ & -1.85484000 & -2.51369100 & -0.38344000 \\
\hline $\mathrm{C}$ & 4.79147000 & -0.91459000 & -0.77919400 & $\mathrm{H}$ & -1.63853500 & -3.23787800 & 0.41627000 \\
\hline $\mathrm{C}$ & 5.22544500 & -0.83700300 & 0.54288500 & $\mathrm{H}$ & -1.23389300 & -2.78600900 & -1.24445600 \\
\hline $\mathrm{H}$ & 4.60455300 & -0.65625900 & 2.59814500 & $\mathrm{O}$ & -1.64885500 & -0.19187600 & -0.98818400 \\
\hline $\mathrm{H}$ & 2.19807300 & -0.62125200 & 2.06683700 & & & & \\
\hline $\mathrm{H}$ & 3.06776700 & -0.93968500 & -2.09488100 & \multicolumn{4}{|c|}{ TS14 ( $\omega$ B97XD optimized) } \\
\hline $\mathrm{H}$ & 5.51862300 & -0.98025900 & -1.58609500 & \multicolumn{4}{|c|}{ imaginary frequency $=-124.88 \mathrm{~cm}^{-1}$} \\
\hline $\mathrm{H}$ & 6.28780100 & -0.84088800 & 0.77607700 & $\mathrm{C}$ & 3.29375600 & 2.42571000 & 0.26336500 \\
\hline $\mathrm{C}$ & 1.01764300 & -0.82573800 & -0.46168800 & $\mathrm{C}$ & 2.54787000 & 1.24967900 & 0.22874600 \\
\hline $\mathrm{O}$ & 0.78106000 & -0.54587600 & -1.70582900 & $\mathrm{C}$ & 3.18903000 & 0.01994600 & 0.04772800 \\
\hline $\mathrm{C}$ & 0.05177600 & -1.10391800 & 0.46827400 & $\mathrm{C}$ & 4.57325400 & -0.01617200 & -0.12387000 \\
\hline $\mathrm{H}$ & -0.79741400 & -0.25677600 & -1.52443400 & $\mathrm{C}$ & 5.31964800 & 1.15641100 & -0.06737500 \\
\hline $\mathrm{H}$ & 0.30456000 & -1.40275000 & 1.48118300 & $\mathrm{C}$ & 4.68000800 & 2.38039100 & 0.12937100 \\
\hline $\mathrm{H}$ & -1.78672100 & 1.50116600 & -0.45894400 & $\mathrm{H}$ & 2.78955600 & 3.38046200 & 0.3891670 \\
\hline $\mathrm{C}$ & -0.65215100 & 3.04996200 & -0.04566200 & $\mathrm{H}$ & 1.46008800 & 1.26332800 & $0.2936440 c$ \\
\hline $\mathrm{C}$ & 0.08853500 & 2.50063900 & 1.18195100 & $\mathrm{H}$ & 5.04793200 & -0.97632700 & -0.30599400 \\
\hline $\mathrm{H}$ & 0.25419000 & 1.42304800 & 1.06691800 & $\mathrm{H}$ & 6.40031700 & 1.12069700 & -0.18438600 \\
\hline $\mathrm{H}$ & 1.06344500 & 2.98792300 & 1.31432000 & $\mathrm{H}$ & 5.26171200 & 3.29853200 & $0.1656660 c$ \\
\hline $\mathrm{H}$ & -0.51276400 & 2.66789300 & 2.08421100 & $\mathrm{C}$ & 2.36627800 & -1.24780200 & -0.05878800 \\
\hline $\mathrm{C}$ & 0.18579800 & 2.82533900 & -1.31169600 & $\mathrm{O}$ & 2.70452900 & -2.09427700 & -0.89418700 \\
\hline $\mathrm{H}$ & 0.41765200 & 1.76281600 & -1.45522000 & $\mathrm{C}$ & 1.22544000 & -1.30297700 & 0.81952300 \\
\hline $\mathrm{H}$ & -0.36946400 & 3.18194200 & -2.18772300 & $\mathrm{H}$ & -0.29291800 & 0.07953400 & -1.21098800 \\
\hline $\mathrm{H}$ & 1.13557800 & 3.37307800 & -1.25659800 & $\mathrm{H}$ & 1.25844200 & -0.63401100 & 1.67671500 \\
\hline $\mathrm{C}$ & -0.94219200 & 4.54139900 & 0.13935700 & $\mathrm{H}$ & -2.29025000 & 0.53439600 & -0.80519500 \\
\hline $\mathrm{H}$ & -1.47101300 & 4.93336700 & -0.73684700 & $\mathrm{C}$ & -3.20230300 & 2.14059200 & -0.16770600 \\
\hline $\mathrm{H}$ & -1.57940000 & 4.69289500 & 1.01832000 & $\mathrm{C}$ & -2.81206000 & 1.88566600 & 1.29913700 \\
\hline $\mathrm{H}$ & -0.01467900 & 5.11060500 & 0.27545800 & $\mathrm{H}$ & -1.83213300 & 1.39715100 & 1.31449400 \\
\hline $\mathrm{O}$ & -1.92013700 & 2.43357700 & -0.17086700 & $\mathrm{H}$ & -2.76785800 & 2.81736000 & 1.88046600 \\
\hline $\mathrm{C}$ & -3.78767900 & -0.71179400 & 0.91552900 & $\mathrm{H}$ & -3.54310200 & 1.21526800 & 1.76758300 \\
\hline $\mathrm{H}$ & -3.98416700 & 0.02152000 & 0.12435100 & $\mathrm{C}$ & -2.17140800 & 3.08829100 & -0.80641700 \\
\hline $\mathrm{H}$ & -4.38636200 & -0.41260800 & 1.78651500 & $\mathrm{H}$ & -1.19310600 & 2.59677900 & -0.77785500 \\
\hline $\mathrm{C}$ & -2.29710300 & -0.66257500 & 1.26179900 & $\mathrm{H}$ & -2.43725400 & 3.27540300 & -1.85386500 \\
\hline $\mathrm{H}$ & -2.01231800 & 0.35499500 & 1.54915500 & $\mathrm{H}$ & -2.10936300 & 4.05150600 & -0.28130500 \\
\hline $\mathrm{H}$ & -2.09292000 & -1.32053400 & 2.11993100 & $\mathrm{C}$ & -4.60517800 & 2.74832700 & -0.24674900 \\
\hline $\mathrm{C}$ & -4.20754700 & -2.10486000 & 0.43554700 & $\mathrm{H}$ & -4.88149400 & 2.91619100 & -1.29428800 \\
\hline $\mathrm{H}$ & -5.27046300 & -2.10914100 & 0.15698400 & $\mathrm{H}$ & -5.33463300 & 2.05624600 & 0.18957100 \\
\hline $\mathrm{H}$ & -4.09432700 & -2.82163200 & 1.26446900 & $\mathrm{H}$ & -4.66161800 & 3.70436400 & 0.29006000 \\
\hline $\mathrm{C}$ & -3.34189600 & -2.56110000 & -0.74318200 & $\mathrm{O}$ & -3.24029400 & 0.93292700 & -0.88118500 \\
\hline
\end{tabular}


C

H

$\mathrm{H}$

C

H

$\mathrm{H}$

C

H

H

C

H

$\mathrm{H}$

C

C

H

$\mathrm{H}$

O

$\begin{array}{rrr}-2.52154700 & -1.94381100 & 1.02940000 \\ -2.83082000 & -0.99765600 & 0.57931600 \\ -3.19342900 & -2.15033100 & 1.87459800 \\ -1.08813400 & -1.81663500 & 1.54989800 \\ -0.98832000 & -0.92404700 & 2.17496100 \\ -0.86943700 & -2.68635700 & 2.19432500 \\ -2.63870700 & -3.05077100 & -0.01999800 \\ -3.66821300 & -3.10102200 & -0.39717200 \\ -2.41743300 & -4.02891500 & 0.43903100 \\ -1.66504800 & -2.79661200 & -1.17138500 \\ -1.90880100 & -1.83512000 & -1.63230000 \\ -1.75679400 & -3.58360400 & -1.93266600 \\ -0.00697000 & -1.80359200 & 0.49007200 \\ -0.21766000 & -2.73493700 & -0.67944500 \\ 0.09334000 & -3.73704400 & -0.34076700 \\ 0.47094100 & -2.47838000 & -1.48763100 \\ -0.88960600 & 0.05327000 & -0.45190700\end{array}$

